\title{
Migrantenselbstorganisationen und ihre Einbettung in theoretische Diskurse
}

\subsection{Definition und Funktionen zwischen Binnenorientierung und Vergesellschaftung}

Es existiert keine offiziell anerkannte, wissenschaftliche Definition des Begriffs „Migrantenselbstorganisation“. Ludger Pries versteht darunter von Menschen mit Migrationshintergrund aufgrund besonderer Lebenslagen, gemeinsamer Interessen und Wertvorstellungen gegründete relativ dauerhafte Kooperationszusammenhänge bzw. Organisationen, ,(1) deren Ziele und Zwecke sich wesentlich aus der Situation und den Interessen von Menschen mit Migrationsgeschichte ergeben und (2) deren Mitglieder zu einem Großteil Personen mit Migrationshintergrund sind und (3) in deren internen Strukturen und Prozessen Personen mit Migrationshintergrund eine beachtliche Rolle spielen“ (Pries 2013a: 1; Herv. i. Orig.). Dabei ist von großer Heterogenität, sehr unterschiedlichen Orientierungen sowie expliziten und impliziten Zielen der MSO auszugehen. Hinsichtlich folgender miteinander verknüpfter Merkmale ist eine immense Vielfalt von MSO auszumachen. In den Blick geraten:

- ihre Größe (von einigen wenigen bis zu Tausenden von Mitgliedern);

- das Einzugsgebiet der Mitglieder und Besucher;

- ihr formalrechtlicher Status als eingetragener Verein (e. V.), als gemeinnütziger Verein, als Körperschaft des öffentlichen Rechts, als Träger (z. B. der freien Jugendhilfe), als Religionsgemeinschaft, als Dachverband, als Einzelorganisation;

- ihre internen (personellen) Strukturen und (Arbeits-)Prozesse mit Blick auf Differenzierung, Spezialisierung, Hierarchie, Steuerung und Formalisierung; die Entscheidungsprozesse, der Informationsaustausch, die Willensbildung 
und Koordination der Mitglieder sowie die Bedeutung des Einsatzes von hauptamtlich und freiwillig-unbezahlt Tätigen (,Ehrenamtlichen“) (4.3.4);

- ihre Ressourcenausstattung und Vermögenswerte durch Mitgliedsbeiträge, Spenden, staatliche Zuschüsse, öffentliche Fördergelder oder die Teilnahme an (inter-)nationalen Programmen;

- ihre Ausrichtung als religiöse, politische, kulturelle, soziale, humanitäre, berufsständische, unternehmerische, Freizeit-, Sport-, Familien- oder Elternvereine, Begegnungszentren, Selbsthilfe- oder Wohltätigkeitsvereine oder Vereine für bestimmte Gruppen;

- ihr Selbstverständnis bzw. ihre Selbstdefinition hinsichtlich einer bestimmten Nation, Ethnie, Religion oder Kultur;

- die Konzentration ihrer Aktivitäten auf das Herkunftsland und/oder die Aufnahmegesellschaft im Sinne einer Herkunfts- oder Aufnahmelandorientierung;

- die sozioökonomischen Merkmale (sozialer Status, Bildung, Alter, Geschlecht) sowie die Herkunftsländer ihrer Mitglieder und Besucher;

- ihre Beziehungen zur Umwelt, wie etwa anderen MSO, der Kommunalpolitik und -verwaltung, zu den Medien etc.;

- ihre „Einflusslogik“, d. h. das Bestreben, gesellschaftspolitische Verhältnisse aktiv mitzugestalten, um (im-)materielle Ressourcen wie Geld und Anerkennung zu erlangen;

- ihre „Mitgliedschaftslogik“: das Bedürfnis nach gemeinschaftlichem Zusammenleben, Informationsaustausch, gemeinsamer Freizeitgestaltung (Sport, Musik, Kultur), Ausübung kultureller und religiöser Bräuche (aus dem Herkunftskontext), z. B. durch das Feiern und Organisieren von Gottesdiensten, Festen und anderen Anlässen

(Pries 2013a, b; Pries 2010a; Gaitanides 2003; Hunger 2004; Nagel 2013a, b, c; Nagel 2015a; Sezgin 2010; Streeck 2017/[1987] $]^{1}$ ).

MSO sind nicht immer eindeutige Ziele oder Tätigkeitsfelder zuzuordnen, da sich letztere - genauso wie bei einheimischen deutschen Organisationen - überlappen und die MSO variierende, multiple Funktionen und Unterstützungsleistungen für unterschiedliche Zielgruppen übernehmen können (I. 2.2). Angesichts der Vielfalt und Kombinierbarkeit der oben genannten Kriterien sind MSO in der Regel nicht als homogene, monolithische und statische Vereinigungen zu betrachten. Veränderungen finden statt, z. B. durch Generationswechsel oder durch Reaktionen auf sich wandelnde Umwelten. Dadurch können MSO auch enorme Dynamiken und

\footnotetext{
${ }^{1}$ Siehe für eine Zusammenstellung von Merkmalen der MSO auch Klie 2018: 469 f.; BMFSFJ 2017: 209.
} 
Wandlungsfähigkeit aufweisen (Pries 2010a: 22, 25; Pries/Sezgin 2010a, 2012). ${ }^{2}$ Die Festlegung , migrationsspezifischer Themen- bzw. Aufgabenschwerpunkt ${ }^{*}$ als ein Definitionskriterium für MSO ist daher zu eng gefasst. Auch bei den Zielgruppen der MSO handelt es sich nicht allein um Menschen mit Migrationshintergrund (u. a. Priemer et al. 2017: 44; I. 2.1). Daher wird in dieser Arbeit von einer breiten Definition von „Migrantenselbstorganisationen“ ausgegangen: Es soll ausschlaggebend sein, dass sie von zugewanderten Menschen oder deren Nachfahren gegründet wurden und dass mindestens die Hälfte der Mitglieder und Funktionsträger einen Migrationshintergrund aufweisen (s. auch Waldrauch/Sohler 2004: $40 \mathrm{ff}$.). Diese Definition lässt völlig offen - und das erscheint für eine realistische Herangehensweise wichtig -, welche Themen und Aufgaben dabei relevant werden können.

Angesichts der Breite ihrer Eigenschaften und Funktionen werden MSO in der fachwissenschaftlichen und öffentlichen Diskussion hinsichtlich ihrer Bedeutungen für die Integrationsleistungen ambivalent und tendenziell polarisiert wahrgenommen. Die zentralen Fragen lauten: Sind ihnen eher integrative oder segregierende Wirkungen zuzuweisen? Und: Inwieweit können MSO (Pro-)Motoren gesellschaftlicher Teilhabe ihrer Mitglieder sein? ${ }^{3}$

Wesentliche Ausgangspunkte des wissenschaftlichen Diskurses über die MSO und ihre Integrationspotenziale bilden das von Friedrich Heckmann (1981, 1992, 1998, 2015: 286-288) geprägte wissenschaftliche Konzept der „ethnischen Kolonie“" sowie die von Georg Elwert (1982) stark gemachte Hypothese der „Binnenintegration“. Beide Ansätze wurden in der sogenannten „Esser-Elwert“-Kontroverse in den 1980er Jahren aufgegriffen; die in ihr vertretenen Argumentationsmuster

\footnotetext{
${ }^{2}$ Seitens der in den als „MSO“ bezeichneten Einrichtungen aktiven Personen wird bisweilen kritisiert, dass ihre Organisationen fortwährend als „MSO“ etikettiert oder sie als „Migrantinnen und Migranten“ oder „Menschen mit Migrationshintergrund“ bezeichnet werden, obwohl sie in Deutschland geboren, aufgewachsen und die Bildungslaufbahn absolviert haben und in den Organisationen im Zeitverlauf ethnische Durchmischungen erfolgen (Hunger/Metzger 2013b: 102 f.; Klie 2018: 478). So wurde im Jahr 2015 das bundesweite Netzwerk der ,neuen deutschen organisationen e. V.“ gegründet, dessen Mitglieder sich nicht mehr als ,Migrantinnen und Migranten" bezeichnen lassen wollen (URL: https://neuedeutsche.org/, letzter Abruf am 03.04.2021; s. auch El et al. 2019; Friedrichs et al. 2020: 34 ff.).

${ }^{3}$ In diesem Zusammenhang muss allerdings betont werden, dass sich die Frage nach den Integrationsfunktionen nicht nur in Bezug auf MSO stellt, sondern angesichts eine durch zunehmende kulturelle Heterogenität und religiöse Pluralität gekennzeichneten deutschen Gesellschaft für Selbst- bzw. Non-Profit-Organisationen im Allgemeinen. Den Vereinen und der aktiven Teilhabe ihrer Mitglieder kommt dabei eine zentrale Rolle zu (Zimmer 2007; Vortkamp 2008).
} 
sind nach wie vor relevant. Wenngleich seitens der Autoren für ethnische Kolonien auch ambivalente Entwicklungsmöglichkeiten ausgemacht wurden, war die Debatte doch von polarisierten Erörterungen geprägt.

Im Folgenden sollen zunächst die wesentlichen Überlegungen zur „ethnischen Kolonie" bei Friedrich Heckmann dargelegt werden, um im Anschluss daran die zentralen Argumente der von Georg Elwert (1982) und Hartmut Esser (1986a) in der nach ihnen benannten Kontroverse zu beleuchten. Geradezu leitmotivisch trägt sich die Gegensätzlichkeit der Positionen mit jeweils unterschiedlichen Akzentsetzungen durch die integrationspolitischen, öffentlichen und sozialwissenschaftlichen Diskurse.

\section{Das Konzept der „ethnischen Kolonie“ (Heckmann)}

Friedrich Heckmann zufolge entstehen ethnische Kolonien ,zum einen als institutionelle Antwort auf die Bedürfnisse der Migranten in der Migrations- und Minderheitensituation, zum anderen als ,Verpflanzung ' und Fortsetzung sozialer Beziehungen, die bereits in der Herkunftsgesellschaft existierten" (Heckmann 1992: 98). Neben der Verwandtschaft als essenzielles Strukturmerkmal sind ,weitere Strukturmerkmale ethnischer Kolonien das Vereinswesen ${ }^{4}$, religiöse Gemeinden, politische Organisationen, informelle soziale Verkehrskreise und Treffpunkte, spezifisch ethnische Medien, schließlich eine ethnische Ökonomie“ (ebd.; vgl. auch Heckmann 1981: 215). Diese selbstständig entwickelten (in-)formellen Strukturen ethnisch-migrantischer Selbstorganisation sind in einem bestimmten räumlichen Territorium wie der Nachbarschaft, einem Stadtviertel oder Stadtgebiet vorzufinden. Trotz vorhandener ethnischer Gemeinsamkeiten ist bei der ethnischen Kolonie jedoch nicht von einer homogenen Gruppe auszugehen, sondern vielmehr von mehreren heterogenen Gruppen, die sich nach regionalen, sozialstrukturellen, politisch-ideologischen, religiösen und kulturellen Merkmalen unterscheiden (Heckmann 1992: 110).

\footnotetext{
${ }^{4}$ Heckmann differenziert hinsichtlich der Vereine zwischen fünf Typen: 1.) dem Arbeiterverein als ,eine Form der Selbstorganisierung der Migranten, der [insbesondere in den 1970er Jahren] das Ziel gemeinsamer Interessenvertretung, Solidarität und Geselligkeit verfolgte“ (Heckmann 1992: 103); 2.) dem Elternverein, der sich auf Fragen, Interessen und Probleme der zweiten und dritten Generation im schulischen Kontext konzentriert (ebd.: 104); 3.) dem „Zentrum“, das vielfältige ethnische Interessen aufnimmt und entsprechende Angebote in den Bereichen Kultur, Sport und Geselligkeit bereitstellt; 4.) dem Regionalverein einer spezifischen Minderheitenkultur, in dem hauptsächlich geselligen und volkstümlichen Aktivitäten nachgegangen wird, sowie 5.) dem ethnischen Sportverein als wichtigstem Typus v. a. für Jugendliche und die Jugendarbeit (ebd.: 104 ff.).
} 
Heckmann (1992: 96-116) erläutert Wirkungen und Funktionen ethnischer Kolonien in Bezug auf Individuen und Gruppen sowie Minderheiten- und Mehrheitsverhältnisse im Aufnahmeland. Zu den Funktionen, die im Folgenden expliziert werden, zählt Heckmann 1.) die Unterstützung von neu Zugewanderten, 2.) die Persönlichkeitsstabilisierung, 3.) die Selbsthilfe, 4.) die kulturspezifische und allgemeine Sozialisation, 5.) die soziale Kontrolle sowie 6.) die Interessenvertretung.

Heckmann zufolge bieten Formen der ethnischen Selbstorganisation zuvorderst Hilfe- und Unterstützungsleistungen für neu Zugewanderte: In Situationen persönlicher Verunsicherung und Destabilisierung in der Einwanderungssituation könnten ethnische Kolonien grundlegende Orientierungs- und Existenzsicherungsbedürfnisse befriedigen, indem sie wichtige Informationen und Problemlösungen parat halten, die neue Anforderungen und Schwierigkeiten bewältigen helfen. In einem vertrauten Kontext würden neu Zugewanderten alltagspraktische, kognitive und emotionale Unterstützungen geboten, wodurch ein aus dem Erleben andersartiger, fremder Verhältnisse potenziell resultierender „Kulturschock“ abgefedert werden könne. Die Stabilisierung von Persönlichkeiten und Identitäten könne auch durch die Inanspruchnahme von Angeboten des gemeinsamen Praktizierens der Herkunftskultur (Religion), durch den Besuch von Treffpunkten für geselliges Beisammensein und Kontaktpflege und durch verwandtschaftliche und nachbarschaftliche Beziehungen erfolgen.

Darüber hinaus würden solche kompensierenden und stabilisierenden Wirkungen durch die innerhalb ethnischer Kolonien stattfindende Selbsthilfe ermöglicht, da sich die zugewanderten Menschen zwecks kollektiver Bewältigung von schwierigen Lebenssituationen miteinander solidarisieren und gegenseitig helfen würden. Diese Selbsthilfefunktion habe sich aufgrund fehlender oder mangelhafter sozialbzw. wohlfahrtsstaatlicher Unterstützungsleistungen innerhalb selbstorganisierter ethnischer Strukturen und Sozialbeziehungen als hochbedeutsam erwiesen. Davon unabhängig sei sie ebenfalls relevant für ergänzende Leistungen im Berufs- und Familienalltag, bei der Betreuung von Kindern oder Pflegebedürftigen sowie generell in Hinblick auf Informationsaustausch und -gewinn. Diese Beziehungsstrukturen generierten somit wichtige Ressourcen (soziales Kapital) für individuelles und kollektives Handeln und beförderten das Vertraut-Werden der Zugewanderten mit der neuen Umwelt.

Heckmann betont des Weiteren, dass eine kulturspezifische und allgemeine Sozialisation von in ethnischen Kolonien eingebundenen Individuen in der Generationenabfolge äußerst wichtig sei, und zwar auch für die Existenzsicherung der Gruppe. Der Sozialisationsprozess würde durch aufnahmebedingte Mechanismen zwischen Inklusion und Exklusion bzw. politisch propagierten Integrations- oder Assimilierungsstrategien sowie durch jeweilige Macht- und Ressourcenverhältnisse 
beeinflusst. Darüber hinaus könne sich das Individuum durch seine Mitgliedschaft in einem (ethnischen) Verein allgemeine gesellschaftspolitisch relevante Kompetenzen aneignen und somit persönlich weiterqualifizieren. In diesem Zusammenhang geht Heckmann auch auf die Akkulturation als einen kulturellen Anpassungsprozess ein, der sich durch die individuelle und kollektive Übernahme von Elementen einer anderen Kultur vollzieht. Der Prozess verändere „Verhaltensweisen, Werte[], Normen, Einstellungen, Präferenzen, Denk- und Wahrnehmungsweisen“ sowie Institutionen und Praktiken von Individuen und Gruppen sowohl in der Minder- als auch in der Mehrheitsgesellschaft (Heckmann 1998: 38). Der Akkulturationsprozess stellt Heckmann zufolge ,eine zweite Sozialisation dar, die mit einem starken Lern- und Anpassungsdruck für den Zuwanderer der ersten Generation verbunden ist" (Heckmann 1992: 112). In einer solchen Stresssituation stelle die Einbettung in eine Binnengruppe motivierende und erleichternde Bedingungen, Entlastungs- sowie identitäts- und persönlichkeitsstabilisierende Funktionen bereit.

Durch das Sicherheit und Schutz gewährleistende Eingebunden-Sein in eine ethnische Gemeinschaft sei das Individuum verstärkt sozialer Kontrolle ausgesetzt, sodass abweichendes (deviantes) Verhalten in Form von Missbilligung oder Ausschluss sanktioniert werden könne.

Möglichkeiten der Interessenartikulation bzw. -vertretung für ethnische Minderheiten seien abhängig von individuellen Partizipations- bzw. kollektiven Gruppenrechten und Gelegenheitsstrukturen für Menschen mit Zuwanderungsgeschichte (Ausländern) in der Mehrheitsgesellschaft; aber auch der Anspruch auf Repräsentation sowie die Beeinflussung politischer Interessen von ausgewanderten Landsleuten im Aufnahmeland durch das Herkunftsland spielten eine Rolle (s. auch 2.2). So habe beispielsweise die Geschichte der Einwanderung in Deutschland gezeigt, dass im Herkunftsland existierende politische und gewaltsame Konflikte zwischen politischen Parteien und ihren Anhängern zu (gewalttätigen) Auseinandersetzungen und Spannungen zwischen unterschiedlichen Gruppierungen in ethnischen Kolonien (vgl. Özak/Sezer 1987: 56) sowie zwischen Zugehörigen von Minderheits- und Mehrheitsgesellschaft geführt haben (vgl. auch I. 2.2).

Heckmann resümiert, dass die Schaffung ethnischer Kolonien hinsichtlich eines langfristig erfolgreich verlaufenden Integrationsprozesses ein temporärer „Schonraum“ (Heckmann 1998) für multiple Problemlösungen sei und damit lediglich eine Übergangsinstitution darstellen sollte. Es sei nicht grundsätzlich von selbstgenügsamen oder autonomen ethnischen Kolonialstrukturen auszugehen, da auch Beziehungen zur Aufnahmegesellschaft geknüpft und gepflegt würden und die ethnische Kolonie zusammen mit diesen Mehrfachbindungen eine "Zwischenwelt“ (Heckmann 1992: 115) darstelle. Heckmann zufolge bestünde allerdings insbesondere „bei einer relativen Vollständigkeit der ethnischen Kolonie [...] die Gefahr 
einer ethnischen Selbstgenügsamkeit, die ein für das Aufbrechen ethnischer Schichtung und für soziale Mobilität notwendiges Aufnehmen außerethnischer Kontakte und das Eintreten in einen universalistischen Wettbewerb behindert" (Heckmann 1992: 115). Die im Folgenden noch darzulegende Elwertsche These einer positiven Sozialintegrationswirkung durch Binnenintegration kann laut Heckmann daher nur für die erste Migrantengeneration Geltung beanspruchen, da eine Verfestigung der ethnischen Kolonien im Generationenverlauf die Gefahr der Ghettobildung, von sozialem Abstieg und gesellschaftlichen Konflikten berge (Heckmann 1998: 41).

\section{Die „Esser/Elwert"-Kontroverse}

Aufgrund ihrer Wirkmächtigkeit werden im Folgenden die zentralen Argumente der Protagonisten Georg Elwert (1982) und Hartmut Esser (1986) beleuchtet, die in ihren beiden zu ,Klassikern“ avancierten Aufsätzen „Probleme der Ausländerintegration. Gesellschaftliche Integration durch Binnenintegration“ (Elwert 1982) sowie „Ethnische Kolonien: ,Binnenintegration“ oder gesellschaftliche Isolation?“ (Esser 1986a) formuliert wurden.

Den folgenden Ausführungen voranzustellen ist der Hinweis, dass die beiden Autoren unterschiedliche Integrationsbegriffe verwenden: In Anlehnung an Hoffmann-Nowotny (1973), der Integration allgemein als „Partizipation an der Gesellschaft“ versteht, präferiert Elwert ein sozialstrukturelles, ,kulturfreie[s]“ Konzept von Integration im Sinne der Teilhabe an gesellschaftlichen Gütern und dem Zugang zu aufnahmegesellschaftlichen Statuspositionen (Elwert 1982: 719 f.); kulturelle Dimensionen wie die Übernahme von Sprache, Religion oder Speisekultur der aufnehmenden Gesellschaft werden dabei nicht als Voraussetzungen für Integration verstanden. Hartmut Esser versteht in dem hier fokussierten Beitrag unter Integration - in Abgrenzung zum Begriff der Assimilation - die „,relativ konfliktfreie Existenz von funktionalen Interdependenzen zwischen Bevölkerungsgruppierungen“" (Esser 1986a: 106), die die Abwesenheit systematischer ethnischer, wirtschaftlicher oder religiöser Ungleichheiten impliziere, da solcherlei Ungleichheiten die strukturelle Integration behinderten (ebd.: 106, 115). ${ }^{5}$

\footnotetext{
${ }^{5}$ In späteren Publikationen konzipiert Esser sein Verständnis von Integration entlang interdependenter gesellschaftlicher Bezugsgrößen und Bereiche als kulturelle, strukturelle, soziale und emotionale bzw. identifikative Dimensionen und als vier mögliche Typen von Sozialintegration: multiple Inklusion (bzw. Mehrfachintegration); Assimilation; Segmentation; Marginalität. Diese realisieren sich jeweils in Abhängigkeit der Inklusion der Menschen im ethnischen Kontext und/oder in der Aufnahmegesellschaft. Esser schätzt die Möglichkeit der multiplen Inklusion jedoch als faktisch unwahrscheinlich ein und betrachtet für eine
} 
Unter dem Begriff der „Binnenintegration“ versteht Elwert einen „Zustand, in dem für das Glied einer durch emische (kulturimmanente) Grenzen definierten Subkultur der Zugang zu einem Teil der gesellschaftlichen Güter einschließlich solcher Gebrauchswerte wie Vertrauen, Solidarität, Hilfe usw. über soziale Beziehungen zu anderen Gliedern dieser Subkultur vermittelt ist“ (Elwert 1982: 720). Seine an diese Definition anknüpfende, auch bereits von anderen Forschenden ${ }^{6}$ hervorgebrachte Ausgangsthese lautet, dass eine starke Integration von Einwanderern in innerhalb der Mehrheitsgesellschaft bestehenden eigenen Sozialstrukturen - als einer „Binnenintegration“ - ,unter bestimmten Bedingungen ein positiver Faktor für ihre Integration in eine aufnehmende Gesellschaft" (ebd.: 718; Herv.: d. Verf.) sein kann. Zur Plausibilisierung seiner Behauptung verweist Elwert auf den Zusammenhang der Ausbildung von Gruppengrenzen und Solidarität in durch Migration und fremdkulturelle Einflüsse bedingten sozialen Stresssituationen: Die Grenzziehung sei eine notwendige Voraussetzung für die Entstehung von Solidarität in der psychisch belasteten Gruppe.

Elwert nennt drei seiner Ansicht nach bedeutsame Aspekte, die sich als positive Effekte aus dem Zusammengehörigkeitsgefühl ergeben und die für die Eingliederung in die Aufnahmegesellschaft bedeutsam sind: 1.) die Stärkung des persönlichen Selbstbewusstseins; 2.) die Aneignung von Alltagswissen und 3.) die Schaffung von Interessengruppen (Pressure-Groups) (ebd.: 721).

Selbstbewusstsein werde primär in Gruppen ausgebildet, die sich in Bezug auf Status und kulturelle Identität ähnelten. Selbstsicherheit ermögliche individuelle Handlungsfähigkeit und eine bewusste Auseinandersetzung mit der fremden Umgebung, die auch das Risiko umfasse, etwas falsch zu machen. Zudem versetze Selbstvertrauen das Individuum in die Lage, Stigmatisierungen abzuwehren. ${ }^{7}$

Alltagswissen betreffe das Wissen, ,wie etwas läuft“ (ebd.: 722), und diene dem desorientierten Individuum dazu, in einer fremdkulturellen Umwelt unterschiedlichste Situationen zu bewältigen. Interessengruppen arrangierten gezielte

langfristige Integration in den Aufnahmekontext die Assimilation, die die Aufgabe von herkunftskulturellen Bindungen impliziert, als einzig erfolgversprechenden Weg (Esser 2001, 2003, 2008, 2009).

${ }^{6}$ Elwert erwähnt in diesem Zusammenhang die Publikation von Park und Miller (1921), von Wirth (1966/[1928]), von Thomas und Znaniecki (1979/[1918]) sowie von von Bodelschwingh (1955/[1861]).

${ }^{7}$ Unter Verweis auf die Befunde von Elias' und Scotsons Studie The Established and the Outsiders (1965) stellt Elwert heraus, dass diese Abwehr bei einer mangelhaften inneren Kohäsion und fehlenden Kontrolle in der Gruppe misslinge. 
Artikulation, Einbringung, Verhandlung und damit auch Durchsetzung von Anliegen. Hier verweist Elwert auf die Möglichkeit der Machtakkumulation aufgrund des inneren Zusammenhalts der Gruppe (vgl. Elias/Scotson 1965).

Elwert betont, dass die Möglichkeit gelingender Binnenintegration in erheblichem Maße von der Offenheit bzw. Aufnahmebereitschaft der Mehrheitsgesellschaft sowie der Bereitschaft zu interethnischer Kontaktaufnahme auf beiden Seiten abhängig sei.

Darüber hinaus führt Elwert drei weitere eingliederungsbedingende Kriterien an (Elwert 1982: 724-726): 1.) die Gewährleistung eines gesellschaftlich bzw. staatlich kontrollierten Gewaltmonopols, das einerseits eine konsistente Strafverfolgung und ein korrektes Verhalten von Polizeibeamten erforderlich mache und das andererseits durch Einwanderer-Communities nicht infrage gestellt werden dürfe - etwa durch den Aufbau eines parallelen Normsystems in Form von mafiaähnlichen Strukturen; 2.) die Verhinderung der Isolierung von Mitgliedern der Einwanderergemeinschaft, wie etwa türkischen Frauen, die das Haus nicht verlassen und lediglich Kontakt zu Familienmitgliedern haben; 3.) die Lern- bzw. Kritikfähigkeit von Individuen hinsichtlich der Gewinnung und Verarbeitung von Informationen über die aufnehmende Gesellschaft, die die Revision und Korrektur von Vorurteilen impliziere. Elwert betont, dass die mit der Binnenintegration einhergehende Markierung und Bewahrung einer eigenständigen kulturellen Identität durchaus mit dem Prozess der Akkulturation kompatibel sei (ebd.: 726). Aus dem Refugium heraus sei eine selbstbewusste und psychisch stabilisierte Kontaktaufnahme zur Außenwelt erst möglich (ebd.: 728 f.).

Hartmut Esser (1986) setzt sich kritisch mit der Elwertschen Binnenintegrationsthese auseinander, wobei er auf den Begriff der ,ethnischen Kolonie“ rekurriert, den Elwert in seinen Ausführungen nicht benutzt. Zunächst rekapituliert Esser die oben dargelegten zentralen Argumente von Elwert. Für die Beurteilung der Integrationspotenziale von binnenintegrierten Strukturen (bzw. ethnischer Koloniebildung) hält er eine differenzierte Betrachtung der unterschiedlichen Eingliederungsformen von Minderheiten für notwendig.

Esser gibt zu bedenken, dass es aufgrund der Betonung von Eigenschaften des kulturellen Andersseins sowie räumlicher Segregation leicht zu Stigmatisierungen und Stereotypisierungen durch die einheimische, sich distanzierende Bevölkerung komme. Gleichwohl sei es möglich, dass - insbesondere in der ersten Phase der Einwanderung - das homogene Milieu der ethnischen Kolonie, als ein „Moratorium“, die Persönlichkeit stärke (Esser 1986a: 111, 113 f.). Auch reflektiert Esser den Gedanken des Eingebundenseins in eine ethnische Gemeinde als Voraussetzung für die Integration in die Aufnahmegesellschaft: Das Vorhandensein eines Rückzugsortes stimuliere durchaus risikobehaftete Versuche interethnischer Kontaktaufnahme 
insofern, als dass bei Misserfolgen weiterhin die ethnische Kolonie als Rückzugsort und Auffangbecken zur Verfügung stünde (ebd.: 112). Als besonders problematisch betrachtet Esser allerdings, dass die Aneignung wichtiger Qualifikationen sowie interethnische Kontakte aufgrund mangelnder Opportunitäten und benachteiligender Strukturen zumeist ausblieben. Esser verweist in diesem Zusammenhang auf die „ethnische Mobilitätsfalle“ (Wiley 1967), die eine außerethnische zugunsten einer innerethnischen Karriere unwahrscheinlich mache. Letztere zementiere wiederum die ethnische Schichtung (ebd.: 113).

Da in der räumlichen Segregation häufig sowohl einheimische als auch zugewanderte sozial benachteiligte Personen aufeinanderträfen, werde die Verschiedenheit häufig als ,provozierende Bedrohung“ (ebd.: 110) wahrgenommen. Dadurch würden Rückzug in die selbstorganisierten Strukturen und interne Milieubildung forciert, mit der Folge von Distanzierung und Schließungstendenzen. Es ist diese „Selbstverstärkungs-Hypothese“, die wechselseitige „Verstärkung von sozialer Distanzierung und kultureller Segmentation“ (ebd.: 114), die Esser an den integrativen Folgen der ethnischen Koloniebildung die größten Zweifel haben lässt. Ihm zufolge habe das für die ethnische Kolonie charakteristische Element der Selbstgenügsamkeit mit der einhergehenden Gefahr einer „Abschottung “8 hochrelevante strukturelle Folgen, da Beteiligung innerhalb der Aufnahmegesellschaft und damit die Aneignung erforderlicher Qualifikationen sowie Aufstiegsaspirationen ausblieben: ,[E]ine strukturelle Eingliederung [wird] nur unter Entfremdung von der ethnischen Kolonie möglich sein" (ebd.: 116).

Zusammenfassend lässt sich sagen, dass Elwert die positiven Wirkungen einer identitätsstabilisierenden „Binnenintegration“ herausstreicht, die langfristig auch die rechtliche und soziale Integration ermögliche. Hingegen sieht Esser durch die Einbindung von Immigranten in eine selbstorganisierte, sich selbst genügende ethnische Subkultur aufgrund von Abschottungstendenzen die strukturelle Integration der Zugewanderten gefährdet.

Heckmann nimmt hinsichtlich der Positionen von Elwert und Esser eine Art Zwischenposition ein, indem er viele für das Individuum und die Gruppe essenziellen Wirkungen und Funktionen der ethnischen Kolonie im Aufnahmeland herausstellt, aber die Realisierung erfolgreicher Integration durch Binnenintegration nur für die erste Einwanderungsgeneration für möglich hält. Sowohl Heckmann als auch Elwert vertreten damit die Ansicht, dass eine in selbstorganisierten

\footnotetext{
${ }^{8}$ Unter Verweis auf eine eigene Studie (Esser 1986b) vermutet Esser jedoch, dass soziale Distanzierungen und gruppeninterne Kohäsion eher auf die Effektivität binnenethnischer Normen und nicht auf ,abschottende Reaktionen“ (Esser 1986a: 115) zurückzuführen seien.
} 
ethnischen Netzwerken vollzogene lebensweltliche Sozialintegration von Migrantengruppen, d. h. deren adäquater Einbezug in die größere Gesellschaft, auch die gesellschaftliche Systemintegration im Sinne einer funktionalen Leistungserfüllung der einzelnen Subsysteme untereinander einschließen kann ${ }^{9}$. Insofern kann argumentiert werden, dass den binnenethnischen Strukturen, den Netzwerken und Vereinen, durchaus brückenbauende Funktionen zwischen identitätsstabilisierender Vergemeinschaftung nach innen und Vergesellschaftung nach außen zukommen kann.

Alle drei der hier zitierten Autoren betonen die essenzielle Bedeutung der identitäts- und persönlichkeitsstabilisierenden Funktionen für das Individuum durch seine Einbettung in eine Binnengruppe, die vertraute herkunftslandbezogene Verhältnisse und Praktiken sowie Möglichkeiten der intraethnischen Zusammenkunft und Kontaktpflege offeriert. Angesichts dieser Übereinstimmung soll hinsichtlich des Untersuchungsgegenstandes von nicht-religiösen und religiösen MSO in der vorliegenden Arbeit folgende Hypothese formuliert werden:

H1: Verglichen mit anderen Zwecken stellen die Aufrechterhaltung der Herkunftskultur und die Schaffung eines Ortes der Sozialität sowohl für nichtreligiöse als auch religiöse MSO besonders wichtige Organisationsziele dar.

Die in diesem Kapitel thematisierten ambivalenten Funktionen der MSO können zugespitzt und in einem Schaubild kondensiert werden (Abb. 4.1).

\footnotetext{
${ }^{9}$ Die theoretische Unterscheidung zwischen einer Sozial- und Systemintegration geht auf Überlegungen des englischen Soziologen David Lockwood zurück, der die Begriffe wie folgt definiert hat: „Während beim Problem der sozialen Integration die geordneten oder konfliktgeladenen Beziehungen der Handelnden eines Systems zur Debatte stehen, dreht es sich beim Problem der Systemintegration um die geordneten oder konfliktgeladenen Beziehungen zwischen den Teilen eines sozialen Systems“ (Lockwood 1979: 125; Herv. i. Orig.).

Der Begriff der Sozialintegration umfasst damit das aufeinander bezogene Handeln, die Beziehungen, die soziale Einbettung und Vernetzung von Gesellschaftsmitgliedern. Der Begriff der Systemintegration bezieht sich auf strukturelle (wechselseitige) Abhängigkeiten von gesellschaftlichen Einheiten und deren gemeinsames Funktionieren hinsichtlich der Herstellung gesamtgesellschaftlicher Kohäsion. Nach Lockwood können beide Integrationstypen geordnet verlaufen, aber durchaus auch Konflikte und Disharmonie produzieren; sie sind daher nicht per se mit einem positiven Zusammengehörigkeitsgefühl zu assoziieren.
} 


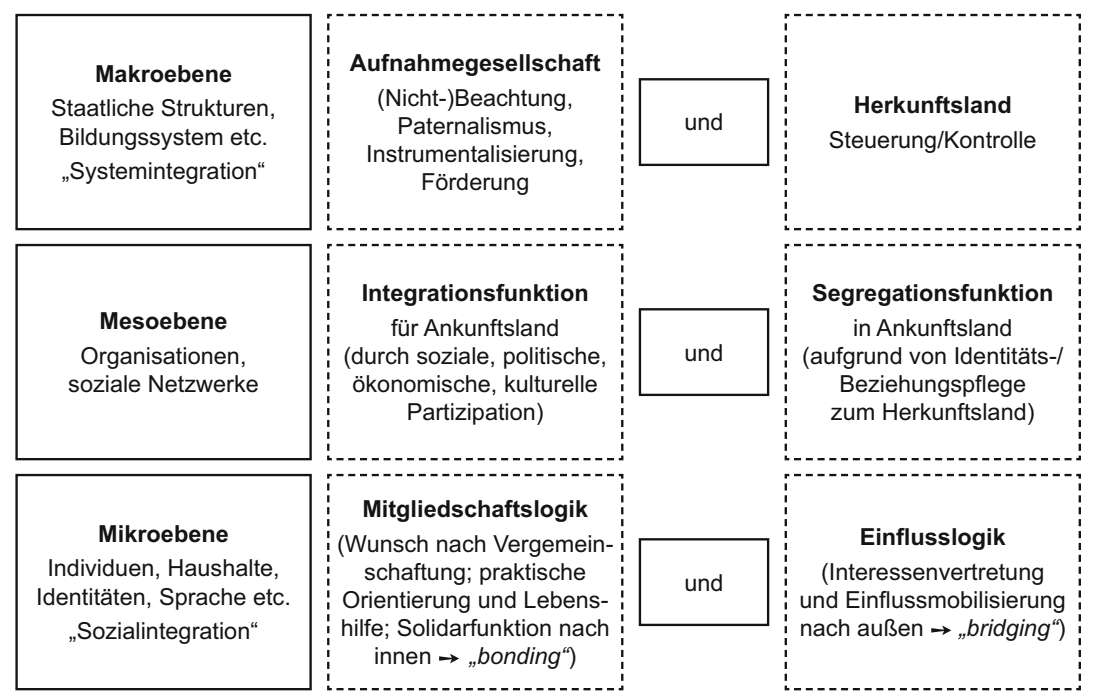

Abb. 4.1 Die ambivalente und polarisierte Wahrnehmung von MSO in Wissenschaft und Gesellschaft (Nach: Pries 2013b. Basis: Elwert 1982; Esser 1986a; Heckmann 1981, 1992, 1998; Fijalkowki/Gillmeister 1997; Thränhardt 1989; Diehl/Urbahn 1998; MASSKS 1999; Thränhardt/Hunger 2000; Halm/Sauer 2005; Hunger 2002; Diehl 2002; Pries/Sezgin 2010a).

\section{Zusammenfassung: Funktionen zwischen Binnen- und Außenorientierungen}

Vor dem Hintergrund der in diesem Kapitel theoretisch aufgemachten Spannungsfelder werden in der einschlägigen Literatur vielfältige Funktionen herausgestellt, die MSO übernehmen können. Ohne Anspruch auf Vollständigkeit lassen sie sich abschließend wie folgt zusammenfassen:

- Allgemeine Unterstützungs- und Sozialisationsfunktionen für Mitglieder, Zugehörige und neu Zugewanderte im Aufnahmeland;

- Identitätsstabilisierende und kompensierende Wirkungen durch vertraute Verhältnisse in einer Minderheitensituation und einer als fremd wahrgenommenen Umwelt;

- Förderung der Ausbildung individueller und kollektiver Identitäten;

- Stärkung kollektiver Handlungskompetenzen und eine damit einhergehende Erhöhung der Selbstbestätigung und Selbstwirksamkeitserfahrung;

- Orientierung ,nach innen“ (,ethnische Binnenintegration“), die einerseits der subjektiven und kollektiven Identitätsstabilisierung dienen und die rechtliche 
und soziale Integration fördern kann, die sich andererseits aber auch als eine segregationsfördernde, mobilitätsbehindernde „Falle“ (,ethnic mobility trap“) erweisen kann (Wiley 1967);

- Scharnierfunktion zwischen Vergemeinschaftung nach innen und Vergesellschaftung nach außen und damit mögliches Bindeglied zwischen Sozial- und Systemintegration (Pries 2010a: 37, 49);

- Ausweitung sozialer Kontrolle und damit Prävention von Devianz (Abweichung von der Norm);

- Kontaktbörsen und Informationsplattformen, Schaffung von sozialen Netzwerken und sozialem Kapital und damit wichtig für Erstintegration ${ }^{10}$;

- Ermöglichung von Ressourcenzugängen (z. B. zum Bildungssystem und Arbeitsmarkt);

- Plattformen für private Selbsthilfe („Empowerment“);

- Schließung von Versorgungs- und Informationslücken durch ehrenamtliche Dienstleitungen und präventive Informationsveranstaltungen z. B. zu den Themen Gesundheit, Erziehung, (Aus-)Bildung;

- Ansprechpartner/Transmissionsriemen für kommunale Politik/Verwaltung, insbesondere auch in Konfliktsituationen aufgrund guter Zugänge zur Community;

- Organe der kollektiven Interessenvertretung und Artikulation politischer Forderungen (,,claims-making“) im öffentlichen Raum bzw. auf kommunal-, landesund bundespolitischer Ebene;

- Einflussnahme auf Entwicklungen in Herkunftsländern, z. B. von demokratischen und entwicklungspolitischen Strukturen;

- Aufbau von Hilfs-, Entwicklungsprojekten und -programmen (humanitäre Krisenhilfe, Entwicklungspolitik, Internationale Solidarität etc.);

- Brückenfunktion zwischen einheimischer (,,alteingesessener“) Bevölkerung mit und ohne Migrationshintergrund und Geflüchteten durch Eröffnung von Erfahrungsräumen und Verdeutlichung der Grundsätze der deutschen Gesellschaft (Verfassungsgrundsätze, insbes. Art. 1 bis 20 GG);

- Vorhaltung von Angeboten als Kontrastprogramm und Immunisierung gegenüber Offerten von integrations- und demokratiefeindlichen Organisationsformen (z. B. von Salafisten oder gewaltbereiten Islamisten $)^{11}$

\footnotetext{
${ }^{10}$ Sozialkapital ist eine Humanressource, die in zwischenmenschlichen Interaktionen ausgebildet wird und abhängig ist von der Zugehörigkeit zu sozialen Netzwerken, von geteilten Werten und Normen sowie verfügbarem sozialen Vertrauen (siehe dazu ausführlich 4.2.1). Mit diesen drei Komponenten können Solidaritätsbeziehungen und Kooperationsbereitschaft zwischen Individuen aufgebaut sowie Ressourcenzugänge hergestellt werden.

${ }^{11} \mathrm{Vgl}$. für entsprechende Zusammenstellungen der vielfältigen Funktionen von MSO auch Klie 2018: 471; BMFSFJ 2017: 209 f.
} 
(Nagel 2015a, 2016; Pries 2010a, 2013a; Uslucan 2015, 2017a; Heckmann 2015, 1998, 1992, 1981; Stolz/Huber 2014; Oswald 2007: 123; Gaitanides 2003: 26, 32; Gaitanides 1992; Fijalkowski/Gillmeister 1997: 29; Esser 1986a; Elwert 1982).

Festzuhalten bleibt, dass durchaus verschiedene und zum Teil auch diametral entgegengesetzte Zielsetzungen und Formen der Arbeit von MSO existieren. Wenn einerseits erforderliche Akkulturationsprozesse, wie das Erlernen der deutschen Sprache oder das Verständnis von modernen Geschlechterrollen, ausbleiben und die Pflege der Herkunftskultur ein zentrales Element in den MSO ist, besteht die Gefahr, dass Deutschland weiterhin und ggf. verfestigend als „Fremde“ erfahren wird. Andererseits gibt es viele MSO, die mit bewusst integrativ ausgestalteter Arbeit Umorientierungen aktiv angehen, sich damit dem Aufnahmeland gewachsen zeigen und ihren Teilhabewillen kundtun.

\subsection{Sozialkapitaltheoretische Blickrichtungen}

Im Rahmen dieser Arbeit werden Eigenschaften und Performanzen von nichtreligiösen und religiösen MSO und deren zivilgesellschaftliche Qualität auf der Mesoebene genauer unter die Lupe genommen. Von besonderem Interesse ist, inwieweit Religion bzw. bestimmte Glaubensrichtungen freiwilliges Engagement stimulieren und damit als Katalysatoren einer aktiven Zivilgesellschaft fungieren können. In diesem Zusammenhang ist der populäre Begriff des Sozialkapitals bedeutsam, dessen zentrale theoretischen und (zivil-)gesellschaftlichen Implikationen für den Forschungsgegenstand der MSO in diesem Kapitel dargelegt werden.

Unter Sozialkapital werden in der Dritte-Sektor-Forschung und im politischen Diskurs Umfang und Qualität des sozialen Zusammenhalts innerhalb einer Gesellschaft verstanden. Sozialkapital gilt als positives, Vertrauensbeziehungen konstituierendes Produkt freiwilligen Engagements, das in zivilgesellschaftlichen Organisationen geleistet wird. Mit dem Begriff werden hohe, zum Teil stark normativ aufgeladene Erwartungen verknüpft (vgl. auch 3.1; 3.2). Mitunter scheint dem Sozialkapitel der Status einer Patentlösung für gesellschaftliche Probleme schlechthin zugesprochen zu werden. Dies tangiert in Sonderheit die integrations-, demokratie- und engagementpolitischen Debatten in Deutschland. In diesen wird die Stabilisierung von Solidarität, Vertrauen und sozialem Zusammenhalt als Medium und Mittel für erfolgreiche Integrationsprozesse und eine 
funktionsfähige Demokratie angesehen (u. a. BPA 2007: 20, 173). Vor diesem Hintergrund erhält das zivilgesellschaftliche Engagement von Menschen mit Zuwanderungsgeschichte und ihren Vereinigungen besondere Aufmerksamkeit.

Dieses Sozialkapital in einer wertepluralisierten Gesellschaft herzustellen, ist jedoch an einige Voraussetzungen geknüpft. Wie im vorigen Kapitel angeklungen ist, ist die Frage, ob die in dieser Arbeit untersuchten Organisationen eher binnenoder außenorientiert agieren, nicht allein von ausschlaggebender Bedeutung. Vielmehr sehen sich die involvierten Personen im gesellschaftlichen Zusammenleben einer Vielzahl von Einstellungen, Zuschreibungen und Verhaltenserwartungen ausgesetzt, die ihre Lebenssituation und Lebensperspektive erheblich beeinflussen können. In diesem Zusammenhang ist Vertrauen, das Personen mit Zuwanderungsgeschichte entgegengebracht wird bzw. das sie selbst aufbringen, als eine zentrale soziale Größe zu betrachten, die in zwischenmenschlichen Beziehungen wie ein Schmiermittel wirkt, das soziale Interaktionen ermöglicht und gesellschaftliches Zusammenleben erheblich erleichtert. Soziologische Analyse muss das Vorhandensein oder Nicht-Vorhandensein von Vertrauen einkalkulieren, kann zumindest nicht davon abstrahieren, soweit ihre Aufgabe darin besteht, gesellschaftliches Zusammenleben zu begreifen.

Diskriminierungs- und Minderheitenerfahrungen, Bedrohungsgefühle, bestimmte (religiöse) Haltungen und Einstellungen sind daher stets mitzudenkende Aspekte. Gleichermaßen ist persönlich erfahrene wechselseitige Anerkennung von großer Bedeutung; das Wissen um organisationale Ressourcen, über die verfügt wird, sorgt für Sicherheit bzw. Unsicherheit bei der Planung und Durchführung vielgestaltiger Aktivitäten. Die bei der Analyse zivilgesellschaftlicher Beteiligung und Orientierungen zu berücksichtigenden Gesichtspunkte tangieren immer auch zwischenmenschliche Beziehungen und sind in der Summe so zahlreich, dass sie - aufs Ganze gesehen - im Rahmen dieser Arbeit empirisch nicht untersucht werden können. Jedoch gehört ihre Kenntnis zur Grundausstattung einer kritischen Reflexion, die den Problemlagen und Debatten der Gegenwart gewachsen sein will. Deshalb liegt in den im Folgenden detaillierter vorgestellten - durchaus auch zu hinterfragenden - theoretischen Prämissen zum Sozialkapital kein abseitiger Exkurs, sondern eine Inanspruchnahme von Überlegungen, mit denen Wahrnehmungen, Beziehungen und das Handeln von Personen und Personengruppen näher erfasst und integrationspolitische Debatten wissenschaftlich verortet werden können.

Im Folgenden werden zunächst die mit dem Terminus Sozialkapital verknüpften grundlegenden Konzeptualisierungen und Hypothesen dargelegt (4.2.1), um im Anschluss daran die darin enthaltene Kernkategorie des sozialen Vertrauens 
näher zu erläutern (4.2.2). Sodann werden die zwei einschlägigen Ausprägungsformen von Sozialkapital - das brückenbildende (,,bridging“) und das gruppenintern bindende (,,bonding“) - behandelt. Hier wird aufgezeigt, dass Sozialkapital sowohl sozial inkludierende positive als auch exkludierende negative Effekte erzeugen kann, die in der Debatte um (des-)integrative Leistungen insbesondere von religiösen und ethnischen Vereinen bedeutsam sind (4.2.3). In diesem Zusammenhang werden auch die von Mark Granovetter (1973) vorgenommene Differenzierung zwischen starken und schwachen Beziehungen herausgearbeitet; ebenso wird die von James S. Coleman (1988, 1990) für die Gewährleistung der Gruppenkohäsion herausgestellte Effektivität von geschlossenen sozialen Netzwerken thematisiert.

Vor dem Hintergrund dieser theoretischen Rahmengebung werden schließlich die Besonderheiten des religiösen bzw. glaubensbasierten Sozialkapitals dargelegt und die in der Forschung zu seiner Produktion in unterschiedlichen - insbesondere christlichen, aber auch islamischen - Konfessionen formulierten Hypothesen referiert, die in Bezug auf den Protestantismus und Katholizismus teilweise diametral entgegengesetzt ausfallen (4.2.4). Abschließend werden die religiösen Gemeinschaften und Organisationen inhärente Ambivalenz und Janusköpfigkeit hinsichtlich ihrer positiven und negativen Eigenschaften und Leistungsstärken betrachtet. Aus ihnen resultiert die Generierung von gesellschafts- und integrationspolitisch anerkannten und eingeforderten Sozialkapitalformen einerseits sowie bemängelten und zurückgewiesenen Ausprägungen andererseits. Damit einhergehende zivilgesellschaftliche, demokratieförderliche Potenziale wie auch unzivile Handlungsformen werden beleuchtet und abschließend im Rahmen des empirischen Teils dieser Arbeit $\mathrm{zu}$ beantwortende Fragen formuliert (4.2.5). Die Ausführungen umfassen relevante konzeptuelle Unklarheiten der diskutierten Ansätze.

\subsubsection{Grundannahmen der Sozialkapitaltheorie}

Die Theorie des Sozialkapitals wurde insbesondere durch die Arbeiten der Soziologen Pierre Bourdieu (1983, 1987), James S. Coleman (1988, 1990) sowie der Politikwissenschaftler Robert Putnam (et al. 1993, 1995a/b, 2000) und Francis Fukuyama $(1995,2000,2001)$ geprägt. ${ }^{12}$ Seit den 1990er Jahren hat sie

\footnotetext{
12 Der Begriff wurde bereits in der Arbeit von Lydia Judson Hanifan (1916) im Kontext der Erklärung des Zusammenhangs zwischen gemeinschaftlichem Engagement und Demokratie verwendet.
} 
eine enorme Konjunktur erfahren und ist in den Sozialwissenschaften zu einem populären Forschungsprogramm avanciert. ${ }^{13}$

Putnams Konzeptualisierung hat einen nachhaltigen Einfluss auf wissenschaftliche Arbeiten unterschiedlicher Fachdisziplinen. Seine Definition von Sozialkapital (Putnam et al. 1993, 1995a/b, 2000) fokussiert nicht das Individuum um seiner selbst willen, sondern die ,Interaktivität“ zwischenmenschlicher Verhältnisse: Sozialkapital ,refers to connections among individuals - social networks and the norms of reciprocity and trustworthiness that arise from them" (Putnam 2000: 19). Diese drei sozialen Dimensionen - Netzwerke, Normen der Gegenseitigkeit und Vertrauen - ermöglichten den Beteiligten koordiniert und effektiv zum gegenseitigen Nutzen und zur Verfolgung gemeinsamer Ziele zusammenzuarbeiten, wodurch auch die ökonomische und politische Leistungsfähigkeit der Gesellschaft insgesamt gesteigert werde (Putnam et al. 1993: 167, 1995a: 67, 1995b: 664 f.).

Mit Blick auf die Zivilgesellschaft als Dritten Sektor stellt Putnams Konzeption von Sozialkapital ein Konstrukt von drei miteinander verflochtenen Dimensionen dar: 1.) die (Ein-)Wirkungsbereiche des Vereinslebens und das sich in den Vereinen vollziehende Engagement, 2.) Normen der Reziprozität bzw. gemeinschaftsfördernde Werte und Normen und 3.) das soziale Vertrauen der Beteiligten untereinander. Aus der Form des Vereinsengagements, das innerhalb der Netzwerkbeziehungen und der sozialen Kontakte entsteht, entwickeln sich - so Putnams Annahme - die beiden anderen Phänomene: das Vertrauen sowie die gemeinschaftlichen Werte und Normen der Wechselseitigkeit (Westle/Gabriel 2008: 5, 12, 162). Soziale Netzwerke und zivilgesellschaftliche Freiwilligenorganisationen ${ }^{14}$ sind nach Putnam für die Funktionsfähigkeit der Demokratie, für gesellschaftspolitische Gestaltungsmöglichkeiten bzw. die Lösung gesellschaftlicher Probleme sowie für die Senkung von Transaktionskosten ${ }^{15}$ essenziell (Putnam et al. 1993: 177; Westle/Gabriel 2008: 41).

Bei Putnam stellt Sozialkapital sowohl ein Individual- als auch ein Kollektivgut dar, das auf der Mikro- und Makroebene Relevanz besitzt (Putnam 2000:

${ }^{13}$ Die zum Konzept des Sozialkapitals international erschienene Literatur hat unüberschaubare Ausmaße angenommen.

${ }^{14}$ Im Kontext der Sozialkapitaltheorie wird häufig der Begriff der Netzwerke verwendet, der im zivilgesellschaftlichen Bezugsrahmen synonym mit den Begriffen freiwillige Assoziationen, Vereine, Verbände, Gemeinschaften etc. und deren Mitgliederzahlen gebraucht wird. Für empirische Analysen stehen zumeist auch keine anderen Daten als zu Vereinen und deren Mitgliedschaftsraten zur Verfügung.

${ }^{15}$ Die für verschiedene Akteure mit der Beschaffung von Informationen, der Organisation, Koordination und Kontrolle verbundenen Kosten. 
19 f., 336 ff., passim; Freitag/Traunmüller 2008; Kriesi 2007), wobei diese beiden Ebenen miteinander verbunden werden könnten. Putnam geht davon aus, dass sich das im Verein(sengagement) zwischen den Individuen produzierte Vertrauen („Beziehungskapital“) positiv auf deren Kooperationsbereitschaft sowie deren politische Einstellungen und Verhaltensmuster auswirkt und damit auch der Gesamtgesellschaft als kollektiver Eigenschaft (,Systemkapital“) zugutekommt. ${ }^{16}$

Putnam verallgemeinert diese Thesen dahingehend, dass durch die Verbreitung demokratischer Werte und Normen die politische Beteiligung in der Gesellschaft steige. Denn das in Freiwilligenorganisationen durch positive Erfahrungen entwickelte zwischenmenschliche Vertrauen bewirke ein geringeres Misstrauen gegenüber politischen Institutionen und Organisationen. Wenn das soziale Vertrauen in offizielle politische Akteure, gewählte Regierungen oder Gesetze auf der Systemebene steigt (,Systemkapitel“), dann müssten politisch Verantwortliche die Bürger hinsichtlich ihres gesellschaftlichen Verhaltens weniger kontrollieren bzw. regulieren (Westle/Gabriel 2008: 20). Anders gesagt: Bei einem hohen Niveau des Sozialkapitals in einer Gesellschaft bedarf es deutlich weniger staatlicher Kontrollzwänge. Putnam et al. pointieren: ,[S]ocial capital [...] is the key to making democracy work“ (Putnam et al. 1993: 185).

Putnams Darlegungen stoßen jedoch auch auf Kritik. Bemängelt wird v. a., dass die zwischen individuellen, kollektiven und gesamtgesellschaftlichen Ebenen unterstellten kausalen Zusammenhänge nicht tiefergehend beleuchtet würden und einer empirischen und theoretischen Fundierung (seinerseits) entbehrten (Westle/Gabriel 2008: 162 f.). Aufgrund der Ausblendung entscheidender Einflussfaktoren würden relevante, deutlich komplexer zu konturierende Rahmenbedingungen stark vereinfacht (siehe zu den Kritikpunkten u. a. Liedhegener/Werkner 2011b; Westle/Gabriel 2008: 157-178; Kriesi 2007; Franzen/Pointner 2007; Kern 2004; Smidt 2003b; Newton 1997). Hinsichtlich der Wirkungszusammenhänge zwischen den einzelnen Sozialkapital-Elementen und der die Mikro- und Makroebene verbindenden Prozesse blieben viele Fragen offen (vgl. auch Westle/Gabriel 2008: 151 f., 169).

Pierre Bourdieu (1983: 185 ff.) ist im Spektrum der Theoretiker zum Sozialkapital v. a. durch seine Kategorie des kulturellen Kapitals (oder Bildungskapitals) bedeutend geworden, ausweisbar z. B. durch entsprechende Schulabschlüsse und Bildungstitel, gewohnten oder adaptierten Umgang mit kulturellen Ressourcen

\footnotetext{
${ }^{16}$ Siehe zum Vorschlag der Einführung der Begriffe des „Beziehungs- und Systemkapitals" anstelle des Sozialkapitals, die beide die in diesem Kapitel noch näher zu erläuternden strukturellen (Netzwerke) und kulturellen (Werte, Vertrauen) sozialkapitaltheoretischen Komponenten umfassen: Westle/Gabriel 2008: 18 ff.; Esser 2000: 241; Gabriel et al. 2002: $25 \mathrm{ff}$.
} 
wie Literatur, Theater oder Musik. Bourdieu hat das „Bild der sozialen Welt“ (Jurt 2003: 76) erweitert. Ihm zufolge kann man Strukturen und Funktionen gesellschaftlicher Bezüge nur adäquat verstehen, wenn man den Begriff des Kapitals nicht nur im ökonomischen Sinn, sondern umfassend, in allen seinen Erscheinungsformen“ begreift (Bourdieu 1983: 184; Herv. weggel.). Bourdieu unterscheidet daher drei grundlegende Typen von Kapital (und damit: Macht) (ebd.: 185), die die sozialen Akteure zu kontrollieren und zu akkumulieren trachten: das ökonomische Kapital (Geld), das kulturelle Kapital (oder Bildungskapital), das z. B. in Form schulischer Abschlüsse und Bildungstitel oder durch die Entwicklung des ,guten Geschmacks“ aneignungsfähig ist, und das soziale Kapital. Unter Sozialkapital versteht er die „Gesamtheit der aktuellen und potentiellen Ressourcen, die mit dem Besitz eines dauerhaften Netzes von mehr oder weniger institutionalisierten Beziehungen gegenseitigen Kennens oder Anerkennens verbunden sind; [...] [E]s handelt sich dabei um Ressourcen, die auf der Zugehörigkeit zu einer Gruppe beruhen" (ebd: 190 f.; Herv. i. Orig.). Das in soziale Beziehungsnetze eingebettete Reservoir an Ressourcen - beispielsweise in Form von Unterstützungsleitungen, Informationsgewinnung, wechselseitiger Anerkennung - lässt sich bei Bedarf für individuelles oder kollektives Handeln (zu persönlichen Gunsten) nutzen. Im Gegensatz zu anderen Protagonisten der Theorie betrachtet Bourdieu Sozialkapital als eine Individuen zur Verfügung stehende Ressource, die das individuelle ökonomische und kulturelle Kapital erhöhen, aber damit auch wesentlich zur Verstärkung gesellschaftlicher Ungleichheitsstrukturen beitragen kann. Bei Bourdieu kann die Relevanz des Aspektes der Reziprozität jedoch relativiert werden. Es zählt der Rückgriff auf soziale Beziehungen und deren Nutzbarmachung, wobei eine Person, z. B. durch ihre Herkunft und soziale Lage, Sozialkapital bereits besitzt oder dieses durch ihre Beziehungsarbeit auszubauen gewillt ist.

Im Unterschied zu Putnam versteht James S. Coleman Sozialkapital als eine informelle ,sozialstrukturelle Ressource“ des individuellen Handelns, die in den „Beziehungsstrukturen“ zwischen Personen enthalten ist und die bestimmte Handlungen von Individuen sowie das Erreichen bestimmter Ziele erleichtere und ermögliche (Coleman 1988: 98, 1990: 302, 1991: 392). Coleman (1988) akzentuiert drei Komponenten von Sozialkapital: 1.) Verpflichtungen und Erwartungen, 2.) soziale Normen (Regeln), effektive Sanktionen und 3.) Informationskanäle, die sich günstig auf die Bildung der anderen beiden Kapitalformen - des Finanz- 
oder Sach- und insbesondere des Humankapitals ${ }^{17}$ - auswirkten. Für die Ausprägung der handlungsbegünstigenden Beziehungsstrukturen als Sozialkapital ist nach Coleman v. a. die Orientierung an sozialen Normen bedeutsam, die durch Sozialisations- und Identifikationsprozesse sowie Sanktionen (in Form sozialer Kontrolle) eingehalten werden und die über spezifische soziale Verbindungen hinaus wirksam sein können (Coleman 1988: 104 f., 1990: 292 ff., 1991: 380, 382 f.). Wenn sich gemeinsames Handeln als erfolgreich erweist, können die beteiligten Personen als vertrauenswürdig eingestuft werden. Dies begünstigt Kooperationen unter den Mitgliedern des betreffenden Netzwerkes und erleichtert Interaktionen auch mit anderen Personen (Coleman 1988: 102, 1990: 310 f., 1991: 396 f.).

Bei Coleman fungiert Sozialkapital als eine unabhängige Variable für die anderen Kapitalformen und als individuelle Ressource. Coleman geht damit vom einzelnen Akteur aus, der sich - allein oder zusammen mit anderen - Ziele setzen, Aufgaben stellen und Projekte in Angriff nehmen kann. Die Akteure verfügen hinsichtlich ihrer individuellen ,Ausstattung' über unterschiedliche Ressourcen, die sie - darin Bourdieu ähnlich - optimieren könnten. Sozialkapital kann aber auch ein öffentliches, kollektives Gut darstellen, aus dem einzelne Personen, auch wenn sie nicht zu seiner Entstehung beigetragen haben, Nutzen ziehen, es aber nicht in Besitz nehmen können.

Francis Fukuyama definiert Sozialkapital vom Resultat her, als einen „Bestand informeller Werte und Normen, die alle Mitglieder einer Gruppe teilen und die Kooperation zwischen den Mitgliedern in der Gruppe ermöglichen [...]“ (2000: 32). Fukuyama betont die zentrale Bedeutung von prosozialen, vertrauens- und kooperationsfördernden (religiösen) Werten und Normen für die Produktion von Sozialkapital, die auch für die wirtschaftliche Prosperität eines Landes ausschlaggebend sei. Zudem stellt er den relationalen Charakter von Sozialkapital heraus, das nicht einfach tradiert und weitergegeben werden könne, sondern das ,vielmehr jederzeit von den Menschen geschaffen [wird], wenn sie ihrem alltäglichen Leben nachgehen“" (Fukuyama 2000: 195).

Die dargelegten Konzeptualisierungen von Sozialkapital sind insofern kompatibel, als sie Sozialkapital als eine relationale Ressource charakterisieren, die im Gegensatz zum ökonomischen und kulturellen bzw. Humankapital in sozialen Beziehungen entsteht und über die kein Akteur allein die Verfügungsrechte ausüben kann. Sozialkapital wird als ein mehrdimensionales Konzept gedacht, das - im Sinne eines „Vitamin B“ (Braun 2002) - als eine für das individuelle und

${ }^{17}$ Coleman (1988: 100 f.) versteht unter Humankapital angeeignete Fertigkeiten und Wissensbestände einer Person, die Voraussetzung für ihre Handlungsfähigkeit sind. Bildung, berufliche Position und ökonomische Ressourcen gehören bei Coleman zum Humankapital, das sich damit vom Begriff des kulturellen bzw. Bildungskapitals bei Bourdieu unterscheidet. 
kollektive Handeln nutzbare Ressource aufzufassen ist, die in auf Dauer gestellten zwischenmenschlichen Interaktionen ausgebildet wird. Sie ist abhängig von der Zugehörigkeit zu sozialen Netzwerken (Beziehungsstrukturen), von als verbindlich betrachteten, geteilten Werten und (Reziprozitäts-)Normen (Ausnahme: Bourdieu) und der Verfügbarkeit (generalisierten) Vertrauens. Solidaritätsbeziehungen und Kooperationsbereitschaft können so ermöglicht und aufrechterhalten und gesellschaftliche Koordination kann erleichtert werden (vgl. auch Enquete 2002: 34).

Angeklungen und herauszustellen ist, dass die Entstehung von Sozialkapital auf strukturellen und kulturellen Komponenten basiert. Dabei umfassen die zivilgesellschaftlichen sozialen Netzwerke in Form ihrer Anzahl, Dichte und der Beziehungstypen die strukturellen Opportunitäten, und die (Reziprozitäts-)Normen, Werte und das zwischenmenschliche Vertrauen betreffen die abstrakteren kulturellen Grundlagen, die auf persönlichen Dispositionen, v. a. Einstellungs- und Wertmustern, der Individuen basieren (Traunmüller 2008: 5 f., 2012: 19 f.; Kriesi 2007: 33-40; Norris/Inglehart 2011: 191-194).

\subsubsection{Soziales Vertrauen als Kernkategorie}

Das soziale Vertrauen stellt innerhalb des Konzepts der Zivilgesellschaft sowie der Sozialkapitaltheorie eine unverzichtbare Kernkategorie dar: Die dauerhafte Einbindung von Gesellschaftsmitgliedern in Netzwerke bzw. soziale Gruppen spielt für die Entstehung von Vertrauen eine zentrale Rolle; ausschlaggebend ist - idealtypisch - die feste persönliche Überzeugung von der Verlässlichkeit einer anderen Person. ${ }^{18}$ Putnam et al. (1993: 170) bezeichnen es als einen wesentlichen Bestandteil von Sozialkapital und Putnam konstatiert: „[T]he more we connect with other people, the more we trust them, and vice versa“" (Putnam 1995b: 665). Putnams Hypothese zufolge fördern Netzwerke zivilgesellschaftlichen Engagements die Entstehung von Reziprozität und generalisiertem sozialen Vertrauen (Putnam 1995a: 67). Die Annahme lautet, dass Gegenseitigkeit und Vertrauen durch die Einbettung von Personen in informelle oder organisierte soziale Beziehungsstrukturen, in denen sie sich näher kennenlernen, face-to-face miteinander

\footnotetext{
${ }^{18}$ Niklas Luhmann zufolge ist Vertrauen „ein elementarer Tatbestand des sozialen Lebens“ und im weitesten Sinne ein Zutrauen zu eigenen Erwartungen (Luhmann 2014/[1968]: 1). Es stellt eine soziale Beziehung dar und bildet sich in einem Interaktionsfeld aus, das durch psychische und soziale Systeme beeinflusst wird (ebd.: 4 f.).
} 
kommunizieren, Informationen austauschen und gemeinsam Entscheidungen treffen, besonders gut aufgebaut und gepflegt werden können (Putnam et al. 1993: 174).

Aus der Perspektive von Putnam basiert die Ausbildung von Vertrauen auf sozialen Netzwerken und Normen, und zwar insbesondere Normen der Reziprozität, die Interaktionen und Kooperationen erheblich erleichtern (ebd.: 172). Reziprozitätsnormen - Normen der Gegenseitigkeit - beschreiben individuelle und gesellschaftliche Erwartungen darüber, dass Hilfe und Unterstützungsleistungen für Personen durch gleich(wertig)e Gegenleistungen der unterstützten oder einer anderen Person erwidert werden. Dabei muss Gegenleistung nicht zwangsläufig umgehend erfolgen, sondern kann auch zu einem späteren Zeitpunkt stattfinden: „I'll do this for you now, without expecting anything immediately in return and perhaps without even knowing you, confident that down the road you or someone else will return the favor" (Putnam 2000: $134^{19}$ ). Die Reziprozitätsannahme zielt auf das Wesen von Solidarität als wechselseitiger Hilfe und impliziert Vertrauen in die Geltung allgemeiner sozialer Normen. Mit ihr einher geht die Produktion von Erwartungen und sozialen Verpflichtungen: „If $A$ does something for $B$ and trusts $B$ to reciprocate in the future, this establishes an expectation in $A$ and an obligation on the part of $B$ " (Coleman 1988: 102; Herv. i. Orig.). Bei Nichteinhaltung dieser Verpflichtungen und gegenseitigen Verhaltenserwartungen ist mit einem Reputationsverlust zu rechnen. Für Coleman ist die ,,prescriptive norm that $[\ldots]$ one should forgo self-interest to act in the interest of the collectivity" (Coleman 1990: 311) entscheidend. In zwischenmenschlichen Beziehungen entstehende Verhaltenserwartungen stiften Vertrauen und Sicherheit: „Wenn die Mitglieder der Gruppe davon ausgehen, daß die anderen sich ehrlich und verläßlich verhalten, dann werden sie einander vertrauen. Vertrauen wirkt wie ein Schmiermittel, welches das Wirken jener Gruppe oder Organisation effizienter macht" (Fukuyama 2000: 32; Herv. i. Orig.). Für Ausbau, Festigung und Aufrechterhaltung von Vertrauen ist wesentlich, dass bereits vorhandenes Vertrauen nicht enttäuscht wird, sondern dass es durch die Kontinuität positiver Erfahrungen - in sozialen Kontakten mit anderen Personen oder Organisationen - bestätigt werden muss (Pickel 2011: 295 f.). ${ }^{20}$

${ }^{19}$ Putnam bezieht sich auf die viel zitierte Parabel von David Hume: ,,Your corn is ripe to-day; mine will be so to-morrow. 'Tis profitable for us both, that I shou'd labour with you to-day, and that you shou'd aid me to-morrow" (Putnam 2000: 135; Hume 1978/[1740]: 520).

${ }^{20}$ An dieser Stelle lassen sich exemplarisch die Begründungen von zwei MSO für ihre NichtTeilnahme an der im Rahmen dieser Arbeit erfolgten Umfrage zitieren: „Was wir bis heute in guter Absicht getan haben, wurde im Nachhinein immer gegen uns verwendet. Nehmen Sie es uns bitte nicht übel. Es geht um Vertrauen und diesbezüglich haben wir in der Vergangenheit 
So wird bei Putnam der strukturelle Faktor der Netzwerkeinbindung zu einer unabhängigen Variablen für die soziale bzw. kulturelle Dimension des Vertrauens: „People who join are people who trust“ (Putnam 1995b: 666). Es wird angenommen, dass durch die Mitgliedschaft in Vereinen kooperatives Verhalten und soziales Vertrauen gefördert werden, wobei letzteres - Putnam zufolge - auf andere gesellschaftliche Bereiche transferierbar, d. h. verallgemeinerbar ist. Dieses generalisierte Vertrauen unterscheidet sich von einem spezifischen in konkreten Situationen mit bestimmten Personen produzierten Vertrauen insofern, als es ein im Zeitverlauf entstehendes allgemeines Gefühl der prinzipiellen Vertrauenswürdigkeit auch gegenüber fremden, unbekannten Gesellschaftsmitgliedern, Institutionen oder Sachverhalten umfasst (Putnam 2000: 136 f.). Laut Putnam stiftet diese verallgemeinerte Erwartungssicherheit in unterschiedlichen Lebenskontexten einen Vertrauensvorschuss, der sich handlungsrelevant auswirkt, etwa hinsichtlich politischer Partizipation oder der Anerkennung allgemein verbindlicher gesellschaftlicher Verhaltensweisen und Pflichterfüllungen (wie z. B. Vermeidung und Verhinderung von Korruption).

In Anlehnung an Mark Granovetter (1973) und dessen Unterscheidung von „,starken“ und „schwachen“ Netzwerken differenziert Putnam zwischen ,dichtem“ und „schwachem“ oder „dünnem“ sozialen Vertrauen (,thick“ und „thin trust"). Dichtes Vertrauen entstehe in engen, familiären Netzwerken, schwaches Vertrauen hingegen eher in loseren, über die eigene Gruppe hinausgehenden und in andere gesellschaftliche Bereiche hineinreichenden Beziehungen (Putnam 2000: 22 f.; Putnam et al. 1993: 171). Für die Produktion von generalisiertem, auch auf unbekannte Personen gerichtetes Vertrauen, wird dem schwachen Vertrauen ein höherer Stellenwert mit positiveren Wirkungen zugerechnet (Putnam 2000: $136 \mathrm{f.})$.

Trotz nicht geringer Überzeugungskraft der Putnamschen Überlegungen zur Entwicklung und Übertragbarkeit von Vertrauen muss an dieser Stelle jedoch auf einige blinde Flecken hingewiesen werden. Denn zweifellos werden einige Einflussfaktoren ausgeblendet, die allerdings mitzubedenken sind und Putnams Fundamentalbehauptungen relativieren. So erscheint es zunächst nicht plausibel und auch nicht empirisch nachgewiesen, dass Vertrauen durch Mitgliedschaft und Aktivität in Vereinen automatisch ansteigt (Levi 1996: 48; Newton 1997: 577; Evers 2002: 65; Freitag 2003: 218). Potenziell könnte es sich auch abbauen oder sogar in Misstrauen umschlagen (siehe hierzu z. B. Geißel/Kern 2000).

schlechte Erfahrungen gemacht“ (Herv.: d. Verf.) — „Ich kenne Sie nicht und werde nicht an der Umfrage teilnehmen. Woher soll ich wissen, was Sie mit den Informationen zu unserem Moscheeverein anstellen?“ 
Inwieweit es in Vereinen zur Ausbildung von gruppeninternem und dann auch transferierbarem Vertrauen kommt, hängt zudem in erheblichem Maße von der Art der jeweiligen Vereinigung, deren personaler Zusammensetzung und insbesondere von deren Zielsetzungen ab (Newton 1997: 581 f.; Stolle/Rochon 1998; Maloney et al. 2008).

Zudem muss hinterfragt werden, ob der Wert des Sozialkapitals innerhalb einer Vereinsstruktur nicht v. a. und in erster Linie dadurch steigt, dass die Ressourcennutzung (materielle, personelle, ideelle) allen gleichermaßen zur Verfügung steht und erst dadurch das Vertrauen in allgemeine Normen des Verhaltens und Urteilens möglich wird (Jansen/Wald 2007: 190). Eine weitere berechtigte Frage besteht darin, ob soziales Vertrauen nicht überwiegend in primären und sekundären Sozialisationsinstanzen - Familie, Schule, Peergroup - erworben wird und viel weniger im Vereinsleben (Newton 1997: 583 f.; Coleman 1988: 109-116). Putnam selbst (1995a: 73, 1995b: 667) hat punktuell auf den hohen Sozialisationswert der Familie wie auch die Relevanz von Bildungsprozessen in den verschiedensten Institutionen hingewiesen, diese Aspekte in seinen zentralen Ausführungen zur Sozialkapitalproduktion aber weitgehend ausgeklammert.

Darüber hinaus stellt sich grundsätzlich die Frage, welche zeitliche Bedeutung und welchen persönlichkeitsbildenden Stellenwert die Vereinseinbindung für das Individuum überhaupt hat und ob nicht in Bezug auf ein individuell vorhandenes Vertrauensniveau davon auszugehen ist, dass noch weit wichtigere Faktoren zu berücksichtigen sind, etwa spezifische Bedingungen der Sozialisation, Strukturen von Gesellschaft und Umwelt (Foley/Edwards 1999; Delhey/Newton 2002, 2004; 4.1). Dabei ist - bevor es überhaupt zu einem Vereinseintritt kommt ein spezifisches Maß an Vertrauen zunächst als eine Voraussetzung für einen Vereinsbeitritt und freiwilliges Engagement und weniger als eine Folge davon einzustufen. Solche empirisch nachweisbaren Selbstselektionseffekte werden bei Putnam ausgeblendet. Kristine Kern schlägt in diesem Zusammenhang eine Relativierung von Putnams oben zitierter These („People who join are people who trust“) durch ihre Umkehrung vor: „People who trust are people who join“ (Kern 2004: 125).

Ein weiterer Kritikpunkt betrifft die behauptete Übertragbarkeit von Vertrauen, das in einem spezifischen Kontext entstanden ist, auf andere gesellschaftliche wie politische Bereiche (Stolle 2002; Newton 1999; Uslaner 2002b: 239). Es wird nicht weiter ausgeführt, welche Mechanismen dafür sorgen, dass spezifisches Vertrauen in ein allgemeines, ,überschwappen“ kann und damit positive Folgen für das ganze demokratische System hat (,Spillover-Effekt": Peterson 1992; Smidt 2003b: 8; Gill 2003: 491). 


\subsection{3 "Bonding“" versus „,bridging social capital“}

Sozialkapital kann sowohl sozial inkludierende als auch exkludierende, negative Effekte erzeugen. Hinsichtlich dieser entgegengesetzten Wirkweisen differenziert Putnam (2000: 22 f.) zwischen einem inklusiven oder ,bridging social capital“ als einem zwischen unterschiedlichen Menschengruppen brückenbauenden Sozialkapital sowie einem exklusiven oder „,bonding social capital“, das ausschließlich interne Bindungen in sich stark ähnelnden, merkmalsgleichen Gruppierungen festigt. Die Differenzierung zwischen Bridging- und Bonding-Sozialkapital ist zu einem wesentlichen, in der Wissenschaft vielfach thematisierten Unterscheidungskriterium geworden ${ }^{21}$, sowohl für die Einschätzung der Wirkungsrichtungen von Netzwerken bzw. Vereinigungen, deren Mitgliederstrukturen und Tätigkeiten (Zmerli 2008, 2003; Hooghe/Stolle 2003; Warren 2001; Putnam 2000; Gittell/Vidal 1998) als auch - und insbesondere - für die Bewertung von (kollektiven) ethnischen und religiösen Zugehörigkeiten bzw. Bindungen (Smidt 2003a; Klein et al. 2004; Borutta 2005; Traunmüller 2012; Pollack/Müller 2013; SVR 2016; BMFSFJ 2017).

Den beiden Ausprägungsformen werden positive und negative soziale Wirkungen zugesprochen. So kann Bonding-Sozialkapital z. B. in dichten Netzwerken ethnischer Enklaven spezifische Reziprozitätsformen stärken, Solidarität mobilisieren und eine wichtige soziale und psychologische Unterstützung für weniger begünstigte Mitglieder der Gemeinschaft darstellen (4.1). Andererseits kann es aber auch bestimmte negative Konsequenzen haben, indem es Fremdgruppenantagonismen und Abgrenzungsprozesse befördert. Das Bridging-Sozialkapital sei hingegen der Kontaktvermittlung zwischen unterschiedlichen Menschen, der Entwicklung umfassenderer Identitäten und Reziprozitäten und damit der gesamten Gesellschaft grundsätzlich dienlicher (Putnam 2000: 22 f.).

Putnam (ebd.) bezieht sich in seinen Ausführungen zur Unterscheidung zwischen Bonding- und Bridging-Sozialkapital auf Granovetters Differenzierung zwischen starken und schwachen Bindungen (Granovetter 1973). Granovetter

${ }^{21}$ Die beiden grundlegenden Formen des Bridging- und Bonding-Sozialkapitals werden gelegentlich um eine dritte Ausprägungsform, und zwar der des ,status-bridging “(Wuthnow 2002) oder ,linking social capital“, ergänzt (Stepick et al. 2009; Woolcock 2001). Diese Form verbindet Gruppen und Organisationen, die über unterschiedliche institutionelle und politische Ressourcenzugänge, Status, Macht und (politische) Einflussmöglichkeiten verfügen. Dabei handelt es sich um eine vertikale Verbindung, beispielsweise zu Führungskräften, Politikern, Wissenschaftlern oder wohlhabenden Personen, während das Bridging-Sozialkapital - dieser Konzeption zufolge - aus horizontalen Beziehungen besteht. „Linking social capital spans vertical arrangements of power, influence, wealth, and prestige. Those with less power acquire influence and other resources through linking social capital“ (Stepick et al. 2009: 16). 
unterscheidet zwischen starken und schwachen Beziehungen und macht im Rahmen seiner Studie zur beruflichen Mobilität auf die „Stärke schwacher Beziehungen" aufmerksam. Seiner These zufolge stellen erweiterte Sozialkontakte (,weak ties“), die über den engen Familien- oder Freundeskreis hinausgehen, für die Arbeitsstellensuche vorteilhaftes Sozialkapital dar, da über entferntere Bekannte, die ihrerseits Zugang zu weiteren Netzwerken und Ressourcen haben, mehr, neue, nicht-redundante und damit bessere Informationen über offene Stellen zu erhalten seien. Starke Verbindungen (,strong ties“), die Freundschaften und Familie umfassten, könnten aufgrund einer geringeren Diffusion von Informationen hingegen eher ähnliche und weniger nützliche Auskünfte bereitstellen. Schwache Beziehungen seien demnach imstande, Netzwerke miteinander zu verknüpfen und eine brückenbildende Funktion zu übernehmen, was bei starken Verbindungen nicht der Fall sei. Granovetters Unterscheidung zwischen starken und schwachen Bindungen scheint auch aufgrund der expliziten Bezugnahme Putnams somit deckungsgleich mit dessen Differenzierung zwischen Bonding- und Bridging-Sozialkapital zu sein.

Starke Verbindungen umfassen also enge familiäre, freundschaftliche oder gruppenspezifische Beziehungen und scheinen aufgrund der Ähnlichkeit ihrer Mitglieder für die Schaffung von Vertrauen und gruppeninterner Solidarität prädestiniert zu sein. Die Merkmalsübereinstimmungen der involvierten Personen basieren dabei auch auf einem Selektionseffekt, denn gemäß dem HomophiliePrinzip ziehen es Menschen vor, soziale Beziehungen und Freundschaften mit ihnen selbst ähnelnden Personen einzugehen (Lazarsfeld/Merton 1954; Brewer 1981; McPherson et al. 2001).

Ein in der Debatte um bridging und bonding wesentlicher Aspekt ist, dass starke Netzwerke zur sozialen Schließung (,closure of social network“) tendieren; sie gewährleisten Vertrauensproduktion, gruppenbezogene Solidaritätsverpflichtungen, Durchsetzung konsentierter Normen, die Senkung von Transaktionskosten und den Aufbau einer kollektiven Identität (Coleman 1988: 99, 1990: 318 ff.). Dies alles kann jedoch auch auf einen innerhalb des Netzwerks existierenden großen sozialen Druck und einen funktionierenden Kontrollapparat zurückzuführen sein. In engmaschigen Beziehungen werden Vertrauensbrüche und Normenverstöße von Abweichlern sichtbar und können sanktioniert werden, z. B. durch den Ausschluss aus der Gruppe. Die Erzeugung von Zwang - im Sinne von Druck und Pressionen - kann das Verhalten des Individuums durch die Androhung oder Anwendung von Sanktionen, Bestrafungen oder auch Gewalt beeinflussen und damit dessen individuelle Freiheit beschränken. Wenn unerwünschte Personen auf Dauer ausgeschlossen werden, kann das die Leistungsfähigkeit der Organisation erhalten. Der Ausschluss kann ein Exempel statuieren und unter den in 
der Organisation verbleibenden Personen Angst erzeugen, indem sie befürchten, dass sie z. B. bei der Verletzung von Verhaltensregeln ebenfalls von einer als vorteilhaft wahrgenommenen Beteiligung ausgeschlossen werden und familiäre oder religiöse gemeinschaftliche Bindungen verlieren (Coleman 1988: 99). Wenn die Vereinigung imstande ist, das Umfeld eines Individuums zu kontrollieren, wird der von der Gruppe ausgehende Druck noch stärker (March/Simon 1976: 71 ff.). In extremen Fällen, wie in fundamentalistischen (Terror-)Netzwerken, Mafiastrukturen oder fremdenfeindlichen Gangs, können Ausstiegsoptionen gar verweigert werden (Jansen 2002: 96). Wenn Protest und andere Formen des Gegenhandelns ausgeschlossen und uneingeschränkte Konformität und Fügsamkeit erwartet werden, können spezifische Erzwingungsinstrumente, wie etwa eine eigene organisationsinterne Gerichtsbarkeit oder Überwachungsinstitution, geschaffen werden, um die Teilnahme an Organisationsaktivitäten und den organisationalen Zusammenhalt sicherzustellen (Kühl 2014: 345). Solche Elemente des Zwangs und der Unterdrückung ermöglichen - bei nicht vorhandener oder erschwerter Austrittsoption - den Nährboden für ausbeuterische Beziehungen. Zudem besteht mit Blick auf die notwendige Stabilität der Gruppe ein hoher interner Bedarf an Konsens, Loyalität und Legitimität von Führern oder Oberhäuptern (Neidhardt 2017/[1979]: 440; vgl. auch Hirschman 197022).

Eine weitere Kehrseite sozialer Schließung besteht darin, dass sie gegenüber anderen Gruppen mit Misstrauen und Abgrenzung und deren Diskriminierung einhergehen kann. In diesem Fall werden unzivile Handlungsweisen bzw. die dunklen Seiten der Zivilgesellschaft offenbar (Levi 1996; Fiorina 1999; Roth 2004). Auch Putnam thematisiert Sozialkapital in Hinblick auf sein negatives Potenzial: „Social capital [...] can be directed toward malevolent, antisocial purposes, just like any other form of capital" (Putnam 2000: 22).

\footnotetext{
${ }^{22}$ Albert Hirschman (1970) hat mit Blick auf die Verbleibsentscheidung in Vereinigungen die Begriffe „Abwanderung“ (exit), „Widerspruch“ (voice) und „Loyalität“ (loyalty) eingeführt. Wenn Mitglieder mit den in der Organisation vorgefundenen Verhältnissen nicht einverstanden sind, können sie ihre Unzufriedenheit entweder durch Abwanderung oder durch Widerspruch zum Ausdruck bringen. Mitglieder mit einer hohen Loyalität werden vor einem Austritt zunächst ihre Kritikpunkte vorbringen, bevor sie wegen Nichtbeachtung ihrer Bemängelungen oder ausbleibender Veränderungen austreten. Dabei geht Hirschmann von einem mechanischen und regulierbaren Verhältnis zwischen ,exit“ und „voice“ aus: Wenn organisationsintern Widerspruch und Protest zugelassen werden, werden Austrittstendenzen reduziert; werden Ausstiege verhindert, ist intern mit mehr Widerstand zu rechnen. Inwieweit diese Sichtweise zutrifft, ist letztlich eine empirisch zu beantwortende Frage.
} 
Vor diesem Hintergrund werden Vereinigungen, die für herkunfts- und wertheterogene Gesellschaftsmitglieder offen sind, die sich mit ihrer gesellschaftlichen Umwelt austauschen und Kontakte zu anderen Gruppierungen pflegen, die erwünschten positiveren Wirkungen zugeschrieben:

„Scholars have argued that bridging is especially important because it promotes a sense of civic responsibility, overcomes divisiveness and insularity, and encourages not only tolerance but cooperation that may be useful for addressing large-scale social problems, such as crime, poverty, and the ill effects of family disruption and inadequate health care" (Wuthnow 2002: 670).

Die brückenbildendes Sozialkapital produzierenden Organisationen trügen damit zur Lösung von Kollektivproblemen (Putnam 2000: 363) und einer vitalen Zivilgesellschaft bei, da sie das Potenzial besäßen, vielfältige Engagementformen und Kooperationen zu stiften, in denen ,soziale Tugenden wie Verantwortung, Verständigung und Vertrauen erlernt werden“ (Enquete 2002: 39) können. Die brückenbildende Ausrichtung komme damit der Produktion sozialintegrativen und demokratieförderlichen Sozialkapitals in Form von generalisiertem Fremdvertrauen der Gesellschaft im Allgemeinen zugute (Offe/Fuchs 2001: 428 ff.; Paxton 2002: 271).

Unabhängig von dem potenziellen Nutzen und dem Selbsthilfecharakter, den belastbare Bindungen innerhalb einer Gruppe (,,ingroup bonds“) für Individuen haben könnten (4.1), wird bei solchen Beziehungen hingegen angenommen, dass sie letztlich exklusive, tendenziell desintegrative Gruppierungen darstellen. Denn die soziale Integration der verschiedenen Segmente in einer Gesellschaft beruhe nicht auf starken Bindungen innerhalb der Gruppe, sondern auf ausgedehnten Intergruppenbeziehungen (Blau/Schwartz 1984: 12 f.). So wird für Vereinigungen, die in ethnischer, sozialer und religiöser Hinsicht homogen sind, das Risiko ausgemacht, dass in ihnen aufgrund sich sozial abgrenzender, Exklusivität beanspruchender Gruppenidentitäten nur partikulares Vertrauen in die eigene soziale Gruppe ausgebildet wird (Gittel/Vidal 1998: 15 f.; Putnam 2000; Putnam/Goss 2001; Lam 2002: 415; Freitag/Traunmüller 2009). Angesichts dieser diametralen Sichtweisen pointiert Siegrid Roßteutscher (2009: 59; Herv. i. Orig.): ,[B]onding macht Gruppen stark, bridging macht sie demokratisch." Bestenfalls balancieren sich beide Merkmale gegenseitig aus (Hadenius 2004: 54).

Im dargelegten Kontext wird für migrantische Vereinigungen als wichtig herausgestellt, dass deren Kooperation und Vernetzung mit anderen gesellschaftlichen Einrichtungen und Organisationen und die dadurch entwickelten 
Vertrauensbeziehungen und Freundschaften für deren Integration in die Residenzgesellschaft und ihre Einflussmöglichkeiten entscheidend verantwortlich sind. So können Beziehungsnetze für die Arbeits- und Wohnungssuche, medizinische Versorgung etc. von großer Bedeutung sein (Pries 1996; Weiss/Thränhardt 2005a; Jacobs/Tilie 2004; Dahinden 2009).

Studien zeigen jedoch zugleich, dass türkische Migranten über größere familien- und verwandtschaftsbezogene Netzwerke als Deutschstämmige verfügen, aber deutlich weniger Beziehungen zu Angehörigen der Aufnahmegesellschaft pflegen (Janßen/Polat 2006; Gestring et al. 2006: 55 ff.; Blasius et al. 2008: 92). Gemäß den oben formulierten Theorieansätzen sollte die Pflege von Verwandtschaftsbeziehungen und intraethnischen Kontakten eher exklusives Bonding-Sozialkapital generieren. Die innerfamiliaren Beziehungen zeichnen sich jedoch positiv durch hohe Solidarpotenziale und gegenseitige Unterstützungsleistungen aus, die ebenfalls sozialintegrative Effekte generieren können (Nauck/Kohlmann 1998; Nauck et al. 1997; Nauck/Suckow 2002; Haug 2003). Vor diesem Hintergrund schlagen Sonja Haug und Sonja Pointner (2007: 385) vor, hinsichtlich der Kontakte zur eigenen ethnischen Gruppe einerseits und Angehörigen der Aufnahmegesellschaft andererseits zwischen einem herkunftslandund einem aufnahmelandspezifischen Sozialkapital zu differenzieren. Letzteres erwachse aus sozialen Beziehungen zu Deutschen und ermögliche Ressourcenzugänge. Vor einer vorschnellen Interpretation, welche von den beiden Ausprägungen mehr und besseres Sozialkapital produziere, sollte jedoch Abstand genommen werden (Haug/Pointner 2007: 389 ff.).

Spätestens an dieser Stelle ist allerdings das Argument anzuführen, dass die konzeptuelle Unterscheidung von Bridging- und Bonding-Merkmalen eine künstliche, konstruierte ist. Vereine, Verbände, Gemeinschaften und deren Mitglieder sollten sich nicht einfach und eindeutig der einen oder anderen Ausprägungsform zuordnen lassen, da angenommen werden kann, dass beide Aspekte in der Realität gleichzeitig auftreten (Curry 2003). So konstatiert auch Putnam: „Many groups simultaneously bond along some social dimension and bridge others. [...] In short, bonding and bridging are not 'either-or' categories into which social networks can be neatly divided, but 'more or less' dimensions along which we can compare dífferent forms of social capital" (Putnam 2000: 23; Herv.: d. Verf.). Unter integrationspolitischen Gesichtspunkten kann das Bonding - in Gestalt der Bewahrung und Pflege der Herkunftskultur - die Gruppe (in der Diaspora) nach innen stabilisieren und somit Voraussetzung für die nach außen gerichtete Handlungsfähigkeit der Gruppe und ihre Teilhabe in der Aufnahmegesellschaft sein. In diesem Sinne stellt Martin Baumann fest: „Erst das Wissen und die Sicherheit eigener Stärke ermöglicht, den eigenkulturellen Rückzugsort und ,Schonraum` zu 
verlassen und aus selbstsicherer Position sich den Anforderungen der Aufnahmebzw. Residenzgesellschaft zu stellen“ (Baumann 2004: 27; s. auch Baumann 2016, 2018).

Hier wird angenommen, dass eine grundlegende Voraussetzung jeder Gruppenbzw. Organisationsbildung zunächst eine Abgrenzung nach außen ist ${ }^{23}$ (s. auch Neidhardt 2017: 436 ff.; Tyrell 1983: 82 f.; Aldrich/Ruef 2006: 4) und Solidarisierung und Konsolidierung der Gruppe nach innen wiederum eine Bedingung für ihre Öffnung nach außen darstellen. Dies impliziert, dass das Gruppendenken und Zusammengehörigkeitsgefühl (,Wir'-Bildung) in der Organisationsinnenwelt mit erwarteten Loyalitäten und Formen der Gegenseitigkeit verknüpft sind. Dieses Phänomen ist insbesondere in Minderheitensituationen von Relevanz. Geht es aber mit Abschließungstendenzen gegenüber der Außenwelt einher, unterbleiben die erwünschten sozialintegrativen und prodemokratischen Wirkungen einer Gruppe. Damit zeigen sich deutliche Parallelen zur Diskussion um mögliche Mobilisierungs- und Empowermentfunktionen der „Binnenintegration“ ethnischer Vereinigungen und resultierender oder ausbleibender sozialintegrativer Wirkungen in der sogenannten „Esser-Elwert“-Kontroverse (4.1; Elwert 1982: identitätsschützende „Binnenintegration“ versus Esser 1986a: „,soziale Distanzierung und ethnische Segmentation"). Es scheint, dass die seit den 1980er Jahren geführte Debatte mit anderen Akzentsetzungen im konzeptuellen Rahmen des inklusiven und exklusiven Sozialkapitals fortgesetzt wird.

Trotz des Einwands, dass die Differenzierung zwischen bridging und bonding problematisch ist, da in Vereinen und Gemeinschaften häufig eine Kombination von beiden Ausprägungsformen vorzufinden ist, wird an ihr als einer zentralen analytischen und gültigen Konzeptualisierung auch in empirischen Studien festgehalten. Dabei ist davon auszugehen, dass verschiedene Arten von Zusammenschlüssen zu unterschiedlichen Niveaus und Formen des Sozialkapitals führen (Maloney et al. 2008). Die herausforderungsvolle und nur empirisch zu beantwortende Frage lautet damit, welche Arten von Vereinigungen unter welchen Umständen mit welchen Auswirkungen auf das Gemeinwesen zwischenmenschliches Vertrauen, kooperatives Verhalten und erweiterte soziale Netzwerke zu fördern imstande sind (Newton 1997: 584; Foley/Edwards 1998; Diani 2004).

Vor diesem Hintergrund belegen Studien zu MSO folgerichtig, dass es nicht legitim ist, generalisierende Aussagen zu Bridging- und Bonding-Performanzen zu treffen, sondern dass diese sich vielmehr fallspezifisch in einer Gemengelage verschiedener Einflussfaktoren und Kontextbedingungen ausprägen. Bridging

\footnotetext{
${ }^{23}$ Per definitionem gehen Identitätsbildungs- mit dezidierten Abgrenzungsprozessen von anderen Individuen bzw. sozialen Gruppen einher (u. a. Pickel 2011: 273).
} 
und bonding werden u. a. bestimmt von Zielsetzungen, Selbstverständnissen, materiellen und personellen Ressourcenausstattungen sowie Strukturen der MSO, von ihrem Verhältnis zum Herkunfts- und Aufnahmeland, der Zeit ihres Bestehens im Aufnahmeland (,Organisationsalter"), vorhandenen (politischen) Opportunitätsstrukturen sowie Wechselwirkungen mit Akteuren in ihren Umwelten und natürlich auch deren Reaktionen und Behandlung (vgl. I. 2.2). ,Erst eine detaillierte Analyse gibt genauere Aufschlüsse über die Zusammenhänge zwischen Assoziationen von Immigranten, Sozialkapital, Integration und Demokratie“ (GeiBel et al. 2004: 12). Eine pauschale Grundsatzdebatte über die Funktionen von MSO ,als Integrationsbrücken oder -fallen ist demnach ad acta zu legen“ (Klie 2018: 473; Herv. i. Orig.; s. auch I. 2.2).

\subsubsection{Religiöses Sozialkapital und seine Produktion in unterschiedlichen Konfessionen}

Angesichts des religiösen Wandels und der in diesem Zusammenhang diskutierten Prozesse der Säkularisierung, Individualisierung und religiösen Pluralisierung (Pollack 2009; Pollack et al. 2012) hat in den letzten Jahren zunehmend das Verhältnis von Zivilgesellschaft, Religion und glaubensbasiertem Sozialkapital (,faith-based social capital“) Forschungsinteressen auf sich gezogen (siehe u. a. Liedhegener/Werkner 2011a; Traunmüller 2008, 2012, 2014; Smidt 2003a; Putnam 2000: 69 ff.). Ein wesentliches Erkenntnisanliegen liegt dabei auf der Frage, inwieweit Religion Vertrauen und gesellschaftlichen Zusammenhalt befördern und damit als Ressource für Zivilgesellschaft und Engagement fungieren kann oder eher konfliktverschärfend wirkt. Für Deutschland stellt sich die Frage insbesondere im Zusammenhang mit muslimischer Religiosität (vgl. auch I. 2.2).

Für den Begriff des religiösen Sozialkapitals können dabei auf der Ebene individueller Religiosität mit der religiösen Netzwerkhypothese und der religiösen Glaubenshypothese gleichermaßen die bereits beschriebenen strukturellen und kulturellen Komponenten geltend gemacht werden (Abb. 4.2) (Traunmüller 2012: 54-66).

Die Frage nach dem in dieser Arbeit relevanten religiösen Sozialkapital setzt - in struktureller Hinsicht - die dauerhafte Einbindung des Individuums in eine religiöse Gemeinschaft, in einen religiösen Verein oder eine Kirche (,,belonging“) voraus, wobei diese wiederum Gelegenheits- bzw. Opportunitätsstrukturen für (öffentliche) religiöse Praktiken (,,behaving“) bereitstellen, wie etwa den Besuch von Gottesdiensten oder die Ausübung religiöser Rituale (Abb. 4.2). Den dadurch entstehenden sozialen Beziehungen und Freundschaften werden hinsichtlich der 
Rekrutierung von Engagierten und Ehrenamtlichen eine bedeutende Funktion auch für andere gesellschaftspolitische Bereiche - zugewiesen (Putnam 2000; Verba et al. 1995).

Auf der Mikroebene spielt hinsichtlich der Generierung von religiösem Sozialkapital die kulturelle Komponente der subjektiven Glaubensdimension (,believing“) eine zentrale Rolle (Abb. 4.2). Religiöse Glaubensinhalte, Überzeugungen und Weltbilder können in unterschiedlichen Graden prosoziale Verhaltensnormen befördern, Mitgefühl und Altruismus erzeugen und Vertrauen aufbauen und damit engagementfördernde und sozialintegrative Wirkungen entfalten (Welch et al. 2004; Yeung 2004; Cnaan et al. 2003; Wuthnow 1991, 1996, 2003; Smith 2003; Offe/Fuchs 2001; Fukuyama 2000; Putnam 2000; Kecskes/Wolf 1996; Stark/Bainbridge 1996; Lenski 1961).

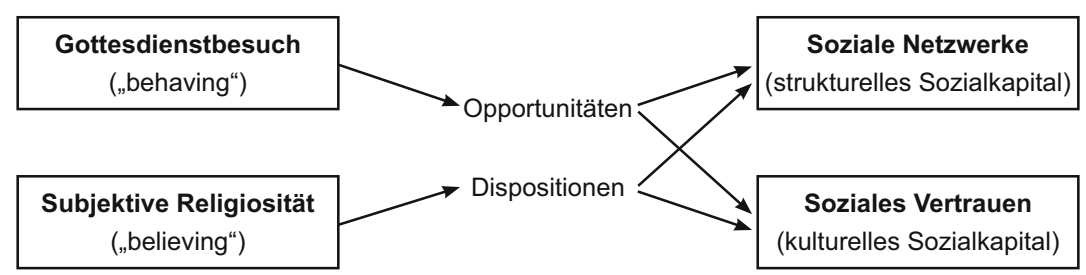

Abb.4.2 Mögliche Wirkungen der Komponenten individueller Religiosität auf Dimensionen sozialen Kapitals (Quelle: Nach Traunmüller 2012: 56).

Mitgliedschaften in religiösen Netzwerken bzw. Organisationen stellen für Klassiker (religions-)soziologischen und demokratietheoretischen Denkens wesentliche Grundlagen für Vergemeinschaftung, Vertrauensbildung und gruppeninterne wie auch darüber hinausgehende soziale und politische Beteiligung dar (Tocqueville 1985/[1840]: 225 ff.; Hidalgo 2011; Durkheim 1984/[1912]; Weber 1988/[1920]; Weber 1985/[1922]: 245-381; Pickel 2011: 75 ff.). Denn in diesen würden Glaubensinhalte, moralische Weltbilder, Werte und Sitten vermittelt und geteilt sowie gemeinsam Rituale (der Empathie und Solidarität) praktiziert. Dementsprechend seien religiöse Organisationen imstande, eine Kultur der Mildtätigkeit und Fürsorge zu schaffen (Wilson/Musick 1997: 696 f.). Putnam konstatiert: „Faith communities in which people worship together are arguably the single most important repository of social capital in America“ (Putnam 2000: 66) und ,[that] it is clear that religious people are unusually active social capitalists“ (ebd.: 67). In diesem Zusammenhang stellt Putnam die Bedeutsamkeit des Gefühls der Zusammengehörigkeit heraus: „,[T]he social 
ties embodied in religious communities are at least as important as religious beliefs per se in accounting for volunteerism and philanthropy. Connectedness, not merely faith, is responsible for the beneficence of church people (ebd., Herv.: d. Verf.; vgl. auch Cnaan et al. 1993; Wilson/Janoski 1995: 137 ff.; Jackson et al. 1995; Wilson/Musick 1997). Putnams Annahmen korrespondieren mit der „Conviction and Community“-Hypothese von Robert Wuthnow (1991: $121 \mathrm{ff}$.), der zufolge Engagement und wohltätige Aktivitäten für Bedürftige insbesondere durch das Eingebundensein des gläubigen Individuums in eine religiöse Gemeinschaft forciert werden. Subjektive religiöse Neigungen allein bewirkten hingegen weniger; relevant seien zum einen der Glaubensinhalt und die innerhalb der Organisation geteilten und verstärkten religiösen Werte und Normen sowie zum anderen die soziale Verbundenheit unter den Gemeindemitgliedern, durch die subjektive Religiosität und Frömmigkeit und damit die Wahrscheinlichkeit wohltätiger Aktivitäten für Bedürftige stiegen. Jenseits des Gottesdienstbesuches können organisierte Formen von Religion also Gelegenheitsstrukturen für die Entstehung von Sozialkapital schaffen (Traunmüller 2008: 20 f.; Pickel 2011: 299; De Hart/Dekker 2005). Allerdings weist Putnam selbst darauf hin, dass religiös motiviertes soziales Engagement einer Person auch davon abhängig ist, inwieweit diese in einen weiteren sozialen Kontext bei der Arbeit, in der Nachbarschaft oder anderen persönlichen Netzwerken eingebunden ist (Putnam 2000: 74). Zudem wird - wie bereits erwähnt - die Entwicklung von zivilem Engagement von der Größe, den Zielen, der Ressourcenausstattung, den Strukturen, der Zeit ihres Bestehens im Aufnahmeland (,Alter“), den Weltanschauungen und der lokalen Verankerung einer jeweiligen religiösen oder säkularen Vereinigung beeinflusst (Geißel et al. 2004: 12; Rauer 2004: 225 f.; Diehl 2004: 248; Berger et al. 2004; Smidt 2003b: 11; Warren 2003; Harris 2003: 137; Curry 2003; Greeley 1997; s. dazu auch 4.3).

Um zu klären, was religiöses Sozialkapital von nicht-religiösem unterscheidet und worin seine potenziellen Vorteile liegen, lassen sich nach Corwin Smidt (2003c: 216-218) vier Charakteristika glaubensbasierten Sozialkapitals heranziehen.

- Erstens könne das auf Basis von religiösem Gedankengut erzeugte Sozialkapital eine dauerhaftere Qualität aufweisen als das aus säkularen Quellen gespeiste. Religiöse Werte könnten andere Bewertungsstandards induzieren und die gläubigen Individuen würden bestrebt sein, diese zu eigen gemachten (internalisierten) Werte und sich daraus ergebende Verpflichtungen als Maßstab zur Beurteilung des eigenen Handelns anzulegen. Smidt zufolge könne 
auch die Häufigkeit des Gottesdienstbesuchs - als Indikator für das persönliche religiöse Commitment, die religiöse Einbindung sowie zentraler Prädiktor für freiwilliges Engagement - auf diese Stabilität und Dauerhaftigkeit des religiösen Sozialkapitals hinweisen.

- Zweitens gäbe die Gegenwärtigkeit transzendenter Werte immateriellen Anreizen wie dem Streben, gottgefällig zu handeln und gute Taten zu vollbringen, den Vorrang und fördere und konserviere die Reziprozität unter Akteuren (s. auch Harris 2003). Die Orientierung an Normen der Reziprozität könne potenziell auch eine bedeutende Grundlage für stärkere Kooperationen darstellen, die in säkular orientierten Gruppen in dieser Form nicht existiere.

- Drittens könne angenommen werden, dass religiöses Sozialkapital eine größere Reichweite als nicht-religiöses hat, da Religionen ein positives inklusives Menschenbild vermittelten und ihre Anhänger dazu anhielten, sich mit Wort und Tat für ihre Mitmenschen einzusetzen. Eigene Vorteile und Interessen bzw. nur zweckrationale Nutzenmaximierung sollten daher hinter den ethischen Imperativen religiöser Werte und Überzeugungen zurücktreten. Das Engagement könnte damit auch Menschen außerhalb der religiösen Gemeinschaft erreichen (vgl. Greeley 1997: 592; Wuthnow 1996). Insbesondere die Mitgliedschaft in religiösen Gruppen könne andere Formen des bürgerschaftlichen Engagements, wie etwa Gemeinschaftsprojekte in der Nachbarschaft oder politische Fürsprache für schwächere, ressourcenarme Menschen, befördern (s. auch Putnam 2000: 67).

- Unter Bezugnahme auf den US-amerikanischen Kontext und Putnam stellt Smidt (2003c: 217) viertens die Quantität der Mitgliedschaften in religiösen, kirchenbezogenen Vereinigungen heraus, die fast die Hälfte aller Vereinsmitgliedschaften ausmachten (Putnam 2000: 66). Das Niveau des religiösen Sozialkapitals sei damit hoch und übersteige das auf andere Art produzierte in deutlichem Maße. ${ }^{24}$

Angesichts obiger Darstellung ist die Bedeutung von Religionen für die Generierung von Sozialkapital nicht zu bestreiten (Wuthnow 2003: 204). Religiöse

\footnotetext{
${ }^{24}$ In Deutschland ist das religiöse Feld mit der Existenz der zwei christlichen Großkirchen deutlich anders strukturiert und mit dem US-amerikanischen Kontext nicht zu vergleichen. In Deutschland scheint das Engagement im kirchlichen Bereich trotz sinkender Kirchenmitgliedschaften jedoch zuzunehmen (Krimmer/Priemer 2013: 39, 43; Vogel et al. 2016a: 131; BMFSFJ 2017: 138). Bei Angehörigen der christlichen Religionsgemeinschaften erweist sich die Verbundenheit mit der eigenen Religionsgemeinschaft als größter Erklärungsfaktor für eine aktive zivilgesellschaftliche Einbindung (Sinnemann 2017: 32 ff.; Ahrens/Sinnemann 2017).
} 
Vereinigungen bieten ihren Mitgliedern wichtige Dienstleistungen, soziale Unterstützung und Netzwerke sowie Gelegenheiten, ihren Glauben zu praktizieren und diesen damit zu plausibilisieren (Berger 1970: 59 ff.; Berger 1969: 45). Religiöse Lehren können die Art und Weise beeinflussen, wie Personen sich selbst und andere Menschen betrachten. Sie können Werte der Aufrichtigkeit, der Hilfeleistung und des Mitgefühls für andere sowie moralische Verpflichtungen gegenüber Familie und Gemeinschaft propagieren. Derlei gemeinschaftlich vertretene und gelebte Qualitäten dürften im Rahmen vorhandener Infrastrukturen mit hoher Wahrscheinlichkeit soziales Vertrauen und gesellschaftspolitisch relevantes Sozialkapital befördern (Offe/Fuchs 2001: $445^{25}$ ). Zudem kann religiösen Zusammenschlüssen das wichtige Potenzial zugesprochen werden, ,zur Reflexion über Werte höherer Ordnung“ (Wuthnow 2003: 207) in der (Zivil-)Gesellschaft und damit zu deren Vitalität beizutragen.

Dieser positiven Einschätzung glaubensbasierten Sozialkapitals lässt sich entgegenhalten, dass von ihm auch negative Wirkungen ausgehen können. Denn religiöse Vereinigungen greifen gegenüber Andersgläubigen oder Außenstehenden zum Teil auch auf Abgrenzungs- und Diskriminierungsstrategien zurück. Aufgrund geteilter Normen und des Willens der Bewahrung innerer Homogenität wirken sie stark identitätsstiftend und bindend (,bonding“) (Pickel 2011: 300, 304; Finke et al. 2006: 624); mit ihrem potenziell exklusiven Charakter können sie auch für innergesellschaftliches Konflikte sorgen (Smidt 2003b: 12 f.) und im Extremfall ein Sicherheitsrisiko bergen (4.2.5). Daher ist hinsichtlich unterschiedlicher religiöser Traditionen bzw. Glaubensinhalte und deren Generierung sozialkapitalrelevanter Dispositionen von erheblichen Varianzen und „kausale[r] Heterogenität" (Traunmüller 2012: 56) auszugehen (ebd.: 56 f.; vgl. auch Koenig 2008: 452 f.; Smidt 2003b: 11).

Das oben Dargelegte legt nahe, dass religiöse Traditionen hinsichtlich ihrer vertrauensgenerierenden Funktionen sowie Ausprägung zivilgesellschaftlicher Strukturen unterschiedlich und v. a. auch widersprüchlich bewertet werden. Auch die Protagonisten der Sozialkapitaltheorie (Coleman, Putnam und Fukuyama) haben - ohne expliziten Anspruch einer umfassenden, systematischen

\footnotetext{
${ }^{25}$ Für Deutschland und die christlichen Kirchen stellen Claus Offe und Susanne Fuchs fest (2001: 445): ,Religiöse Orientierung und Stärke des religiösen Engagements sind mit Sozialkapital positiv korreliert. Kirchen fungieren als Katalysatoren der Aktivität in Vereinigungen. Wenn sie z. B. Räume für die Versammlungen von Umweltschutzgruppen zur Verfügung stellen, fördern sie die Entwicklung kooperativer Kompetenzen und erhöhen das Bewusstsein für soziale Probleme; dies steht in Übereinstimmung mit den Doktrinen und Lehren, die zu einer Beteiligung der Christen an weltlichen Angelegenheiten-aufrufen.“
} 
Analyse verschiedener Konfessionen - die Bedeutung verschiedener Religionen für die Ausbildung von Sozialkapital thematisiert und sind diesbezüglich zu teilweise kontradiktorischen Einschätzungen gekommen. Polarisiert gegenübergestellt werden zumeist der vertrauens- und demokratieförderliche Protestantismus, der aufgrund seiner flachen, dezentralen Strukturen Selbstbestimmung, Selbstverantwortung und freiwillige Selbstorganisationen ermögliche, während die hierarchischen Strukturen des als Weltkirche organisierten Katholizismus persönliche Selbstständigkeit, Verantwortungsübernahme und Partizipation unterdrücke und damit die Bildung von Sozialkapital behindere (Roßteutscher 2009, 2011; Lam 2006; Putnam et al. 1993: 173 f., 244; Fukuyama 1995, 2000; Curtis et al. 2001; Weber 1988/[1920]).

Diese altüberkommene organisationssoziologische These wird flankiert von entsprechenden Hypothesen, dass der Protestantismus mit der Präferenz für Individualismus und für frei gewählte soziale Beziehungen auch außerhalb von Familienstrukturen seine Wirkung entfalte, während der Katholizismus mit einem streng strukturierten Kollektivismus verbunden sei, der traditionell der Pflege familiärer, verwandtschaftlicher Beziehungen den Vorrang gibt (Weber 1988/[1920]: 523; Lenski 1961: 224; Curtis et al. 2001). Diese dichotomischen Verschiedenheiten würden in unterschiedlichem Ausmaß das Interesse der Individuen animieren, sich zivilgesellschaftlich einzubringen.

Den skizzierten Auffassungen wird freilich auch widersprochen. So konstatieren Claus Offe und Susanne Fuchs (2001: 445), dass der römische Katholizismus mehr Sozialkapital produziere als der Protestantismus, ,weil die katholische Doktrin [...] den Gläubigen eine Ethik der Barmherzigkeit sowie ein aktives Interesse für das Wohlergehen der Mitgläubigen in einer Gemeinde einprägt" (vgl. auch Scheuch 1993: 169). Und Coleman schreibt dem kollektivistischen Katholizismus eine sozialkapitalrelevantere Wirkung zu als dem Protestantismus, da dieser mit seiner „Ideologie der Selbstgenügsamkeit [...], die die individuelle Beziehung jedes Einzelnen zu Gott betont, [...] die Schaffung von sozialem Kapital hemmen" könne (Coleman 1990: 321; Übers.: d. Verf.). Andere Autoren stellen das von der katholischen Soziallehre abgeleitete Prinzip der Subsidiarität heraus, das die Verantwortung für gesellschaftspolitische Belange nicht zuvorderst beim übergeordneten Staat, sondern zunächst bei den kleineren (zivil-)gesellschaftlichen Gruppen und Organisationen verorte, sofern diese, kraft ihrer Kompetenzen, zur Übernahme entsprechender Aufgaben in der Lage seien (Manow 2002; Fix 2001).

Es ist evident, dass es „den“ Katholizismus und „den“ Protestantismus nicht gibt. Eher ließe sich z. B. von einer heterogenen protestantischen Konfessionsfamilie sprechen. So weist Putnam auf evangelikale und fundamentalistische 
Gruppen hin, zu denen er u. a. Pfingstler, die Zeugen Jehovas, die Mormonen und die Southern Baptists zählt (Putnam 2000: 76). Für die Evangelikalen konstatiert Putnam individuelle und kollektive nach innen orientierte Aktivitäten innerhalb der eigenen Gemeinschaft und weniger nach außen gerichtete Tätigkeiten wie auch eine niedrigere Wahrscheinlichkeit, in der größeren Gemeinschaft eingebunden zu sein (ebd.: 77 f.; s. auch Wuthnow 2003: 204; Wilson/Janoski 1995: 138, 149 f.; Wilson/Musick 1997: 699; Wuthnow 1999: 338, 346). Entsprechend wird für fundamentalistische Ansichten (im Sinne eines wortwörtlichen Verständnisses der Bibel oder der Prophezeiung des nahenden Weltendes) im US-amerikanischen Kontext festgestellt, dass sie negativ mit sozialem Vertrauen korreliert sind (Putnam/Campbell 2010: 468 f.). Die strengen moralischen Einstellungen und Überzeugen der evangelikalen Protestanten, dass die Welt sündhaft und Gott ein harter Richter ist (ebd.: 113 f.), korrespondiert mit dem Primat des persönlichen Seelenheils im Jenseits, das v. a. der eigenen Gruppe zugestanden wird (ebd.: 534 ff.; Mock 1992: 21) und daher eher mit Abgrenzungstendenzen anstatt mit öffentlichem sozialem Engagement einhergehe (Wuthnow 1999; s. auch Lehmann 2008).

Fukuyama befasst sich im Zusammenhang mit dieser Thematik auch mit nichtchristlichen Religionen. So betrachtet er den chinesischen Konfuzianismus als „eine im Kern familistische Philosophie“, die darauf abziele, ,die Familienbande durch moralische Erziehung zu festigen“, und die „die Beziehungen innerhalb der Familie über alle anderen sozialen Beziehungen“ erhebe (Fukuyama 1995: 78). Diesen Familismus findet Fukuyama auch „,in der katholischen Betonung der Familie" (Fukuyama 2000: 320) in Lateinamerika, Südeuropa und (wie Putnam) in Süditalien vor. Die Folge dieses Familismus sei ein hohes sich auf die eigene Familie konzentrierendes Verpflichtungsgefühl, das sich aber kaum auf außerhalb existierende Kreise beziehe und mit einem geringen Fremdvertrauen einhergehe, sodass „der Grad von Ehrlichkeit und Kooperation im öffentlichen Leben [...] sehr viel geringer als in der Familie [ist]. Vetternwirtschaft und eine alles durchdringende Korruption sind die Folge" (ebd.: 34; vgl. auch Banfield 1954).

Angesichts obiger Hypothesen ist jedoch ein wichtiger Aspekt herauszustreichen: Generell ist hinsichtlich der Schaffung von Sozialkapital die Unterscheidung zwischen religiösen Mehrheiten und religiösen Minderheiten wichtig, da sich laut Traunmüller ,gerade letztere durch eher abgrenzende Formen sozialen Kapitals kennzeichnen lassen sollten“ (Traunmüller 2012: 90). Bezüglich der zivilgesellschaftlichen Verhaltensweisen und Einstellungen der muslimischen Minderheit ließen sich daher für den europäischen Kontext theoretisch fundierte Erwartungen formulieren (ebd.). 
Die Gruppe der Muslime hat in der sozialkapitaltheoretischen Diskussion und der entsprechenden empirischen Forschung bislang wenig Aufmerksamkeit erfahren. Dies hat sich erst in den letzten Jahren verändert (Traunmüller 2008, 2012, 2014; Pollack/Müller 2013), da (organisierte) Muslime als größte religiöse Minderheit im deutschen und europäischen Kontext in der Diskussion um Zuwanderung und Integration in den Fokus gerückt sind. Da für die Gruppe der Muslime eine besonders geringe Engagementbeteiligung und auch für das zwischenmenschliche Vertrauen unterdurchschnittliche Werte festgestellt werden (Pollack/Müller 2013: 48 ff.; Traunmüller 2008, 2012, 2014, 2018; Stadelmann-Steffen et al. 2010), wird bei den Muslimen von einem ,ausgeprägten kollektivistischen Familismus ausgegangen, welcher dem verwandtschaftlichen Zusammenhalt höchste Priorität einräumt“ (Traunmüller 2012: 59; vgl. auch Pollack/Müller 2013: 49 f.; Traunmüller 2008: 9; Gellner 1981: 67) und der eher partikulare, d. h. weniger generalisierte Vertrauensbeziehungen erzeugen dürfte (Traunmüller 2011). Entsprechend konstatiert Traunmüller, dass sich die muslimische Religiosität hinsichtlich des Umfangs der zivilgesellschaftlichen Partizipation von Muslimen als hemmender Faktor erweise, wobei dieser Befund allerdings nicht zwangsläufig mit Abgrenzung von der Mehrheitsgesellschaft gleichzusetzen sei (Traunmüller 2012: 209).

Grundsätzlich bedarf es zukünftig weiterer differenzierter und sorgfältiger Analysen bezüglich der Ursachen für eine geringere Sozialkapitalausstattung der Muslime (Pollack/Müller 2013: 49). Bei diesen Untersuchungen sollte immer auch berücksichtigt werden, dass das niedrigere Sozialkapitalniveau, wie auch bei anderen (religiösen) Minderheiten, im Allgemeinen Folgen gesellschaftlicher Ausgrenzungsmechanismen sein kann. Migrationsspezifische Teilhabehürden, kulturelle Hemmschwellen, fehlende soziale Mobilitätsoptionen und tatsächlich vorhandene oder wahrgenommene Vorurteile, Diskriminierungserfahrungen und Negativ-Diskurse bleiben nicht ohne Auswirkungen auf die Betroffenen und dürften deren (zivilgesellschaftliche) Rückzugs- und Abgrenzungstendenzen forcieren (3.2; Uslucan 2011; Pollack et al. 2016; Uslaner 2002a).

Unabhängig von diesen migrations- bzw. islambezogenen Fragestellungen ist herauszustellen, dass die Untersuchung der Beziehungen zwischen verschiedenen - v. a. nichtchristlichen - Religionen und der Generierung von Sozialkapital (inter-)national bislang wenig systematisch erfolgt ist. 


\subsubsection{Die Janusköpfigkeit und das (un-)demokratische Potenzial religiöser Vereinigungen}

Das oben Dargestellte zusammenfassend lässt sich festhalten, dass religiöses Sozialkapital (Zivil-)Gesellschaft und Demokratie stärken, zugleich schwächen und schädigen kann, wenn sich betreffende Akteure intolerant oder gar demokratiefeindlich gerieren. Diese Doppelgesichtigkeit respektive Janusköpfigkeit soll im Folgenden näher beleuchtet werden.

Es war Alexis de Tocqueville (1985/[1840]: 248 ff.), der (religiöse) Vereine als Basis einer funktionierenden und vitalen Demokratie auffasste und damit einen für künftige Überlegungen wichtigen politikwissenschaftlichen Leitgedanken gestiftet hat. Die essenzielle Bedeutung von Assoziationen (Vereinen) wird damit begründet, dass in diesen bestimmte - Egoismus und Individualismus überwindende - Bürgertugenden (,civic virtues“) und das gemeinschaftliche Eintreten für Angelegenheiten von allgemeinem Interesse eingeübt werden. Ohne ein solches Vereinsleben und ohne aktive Teilnahme der Bürger könne die Demokratie nicht überleben (ebd.: 228). Die über Religionen vermittelten moralischen Weltbilder stifteten Handlungsorientierung und übernähmen eine wichtige demokratieförderliche Rolle, indem sie die Individuen zum Gebrauch ihrer politischer Rechte ermutigten, was für die Funktionsfähigkeit der liberalen Demokratie wesentlich sei (ebd.: 225 ff.). ${ }^{26}$

Die Debatten um Engagementpolitik, Zivilgesellschaft und Sozialkapital sind in den letzten Jahrzehnten insbesondere hinsichtlich der Funktionen von Religion jedoch eher schärfer und kontroverser geworden. Dies hat Wissenschaftler animiert, die programmatische Bedeutung der von den Religionen gestifteten bzw. inspirierten moralischen Positionen herauszuarbeiten bzw. zu erneuern, geradezu als Gegengewicht zu extremen Auffassungen in der Gesellschaft: „Such moral and religious understandings produce both a basic cultural legitimation for a society which is viewed as at least approximately in accord with them, and a standard of judgment for the criticism of a society that is seen as deviating too far from them“ (Bellah 1975: ix; s. auch Cochran 1990: 56 f.; Böckenförde 1976:

\footnotetext{
${ }^{26}$ Im internationalen Kontext befürworten zahlreiche Wissenschaftler die Ansicht, dass es im Grunde das Vereinsleben ist, das Sozialkapital hervorbringt, und es damit letztlich auch die Grundlage für ,wise public policy, robust economic development, and efficient public administration“ (Skocpol/Foriona 1999: 13), ein vitales demokratisches und wirtschaftlich leistungsfähiges System, darstellt. Siehe zur Relevanz des durch das Vereinsleben gebildeten Sozialkapitals für die Beständigkeit und Leistungsfähigkeit von Demokratien u. a. auch Liedhegener/Werkner 2011b; Smidt 2003b, c; Herbert 2003b; Gabriel et al. 2002; Paxton 2002; Böckenförde 2000; Foley/Edwards 1998; Bellah 1975.
} 
60). Dabei bleibt die Frage nach der Fähigkeit von (religiösen) Vereinen, Bürger nicht nur zu sozial ausgerichtetem Engagement, sondern auch politisch zu aktivieren, eine hochaktuelle. Fakt ist, dass die Rolle von Religionen im öffentlichen, demokratischen Leben ein kontrovers diskutiertes Thema ist und bleiben wird.

In der US-amerikanischen Forschung wird betont, dass die Einbindung von Bürgern in religiöse Vereinigungen das Erlernen von Organisations- und Kommunikationsfähigkeiten und damit zivilgesellschaftliche Kompetenzen (,,civic skills“) fördern würde (Verba et al. 1995: 282 f., 317-333, 377-384, 518-521; Wald et al. 2005: 121; Lam 2006; Smith 2003; Putnam 2000). Diese könnten dann auch als Sprungbrett für politische Partizipation der Individuen fungieren (Verba et al. 1995: 330 f.; Peterson 1992; Wald et al. 1993: 130; Putnam 2000: 66 f.). Religiöse Einrichtungen spielten in den USA eine wichtige Rolle bei der Verbesserung der politischen Beteiligung von ressourcenarmen Bürgern (Verba et al. 1995: 18, 320) und übernähmen in ihrer Mobilisierungsleistung häufig unbewusst eine wichtige Funktion des Empowerment (Warner 1993: 1068 ff.). Unter Verweis auf Verba et al. (1995) unterstreicht Putnam (2000: 66), dass Kirchen ,an important incubator for civic skills, civic norms, community interests, and civic recruitment" seien. Religiös aktive Personen lernten, Reden zu halten, Treffen zu organisieren, Meinungsverschiedenheiten $\mathrm{zu}$ beheben und in verwaltungstechnischen Dingen Verantwortung zu übernehmen. Zudem gingen sie Freundschaften außerhalb der religiösen Gruppe ein, was zu ihrer Einbindung auch in ,säkularen ‘ Organisationen und zu anderen Formen des bürgerschaftlichen Engagements jenseits religiöser Gemeinschaftsaktivitäten führe, wie etwa politischer Partizipation in Form des Wählens oder der Beteiligung an anderen Gemeinschafts- und Wohltätigkeitsprojekten (ebd.: 66 f.). Verba et al. betonen in ihrer Argumentation, dass der Erwerb von bürgerschaftlichen Fähigkeiten grundsätzlich abhängig sei von den jeweils gebotenen Opportunitäten und dem sozioökonomischen Status von Individuen und Gruppen (Verba et al. 1995: 319 f.; Brady et al. 1995). Zudem dürfte wahrscheinlich sein, dass sich die im organisationalen Kontext erlernten Verhaltensweisen v. a. dann als für Demokratie und Zivilgesellschaft relevant erweisen, wenn die Strukturen einer Vereinigung offen sind für sich unter Umständen stark unterscheidende Mitglieder: ,[T] he more economically, ethnically, and religiously heterogeneous the membership of an association is, the greater its capacity to cultivate the kind of public discourse and deliberation that is conducive to democratic citizenship“ (Gutmann 1998: 25).

Hinsichtlich der politischen Aktivierung von religiös involvierten Menschen sind solche Hypothesen allerdings nicht verallgemeinerbar (Cassel 1999; Warren 2003; Wood 2003; Harris 2003; Smidt 2003b: 11), sondern von Fall zu Fall zu prüfen. So beteiligen sich beispielsweise die Zeugen Jehovas nicht an politischen 
Wahlen, da sie ,kein Teil der Welt" sind. Sie berufen sich auf unterschiedliche Bibelstellen (z. B. Johannes 6:15; 15:19), die sie als Aufforderung zu einem politisch passiven Verhalten auslegen. Aus ähnlichem Grund lehnen sie auch ab, sich für politische Ämter zu bewerben (vgl. Zillmann 2015: 87 f.; Zurlinden 2015: 120). ${ }^{27}$

Vor diesem Hintergrund werden insbesondere in der US-amerikanischen Fachliteratur jedoch auch weitere spezifische Charakteristika religiöser Organisationen thematisiert, die ihre Janusköpfigkeit erklären helfen und die deutlich machen, warum religiöse Organisationen für die Ausbildung von exklusivem Sozialkapital (bonding) besonders, anfällig' sind. Diesbezüglich herauszustellen ist eine im Vergleich zu nicht-religiösen Vereinigen potenziell größere Werteund Gruppenhomogenität in religiösen Organisationen (Cnaan et al. 2003: 28 f.; Roßteutscher 2009: 59 ff.; Pickel 2011: 300, 304). Der Wertekonsens erleichtert die Produktion von gruppeninternem sozialen Vertrauen, von Normen der Reziprozität und die Etablierung einer organisationsinternen Engagementkultur (s. dazu auch 4.4.2). Religiöse Organisationen sind hinsichtlich ethnischer und sozioökonomischer Merkmale ihrer Mitglieder meist sehr homogen zusammengesetzt (Roßteutscher 2009: 39, 50; Emerson/Smith 2000: 135 f.; Burns et al. 2001: 234), obgleich ihnen das Potenzial zugesprochen wird, Menschen unterschiedlicher Schichten und Generationen anzusprechen und zu repräsentieren (Coleman 1988: 106; Coleman/Hoffer 1987: 215; Verba et al. 1995: 317, 320). Die Homogenität kommt insbesondere durch familiäre Sozialisation, aber auch durch (Selbst-)Selektionsprozesse zustande. Die geteilte religiöse Überzeugung und der gemeinsame Wertehorizont stellen einen selektiven Anreiz für Individuen dar, Mitglied in einer konfessionellen Gemeinschaft zu werden. Deren Charakter und Aktivitäten dürften durch eine bestimmte religiöse Lehre geprägt sein, was in einem ,säkularen' Sportverein z. B. nicht der Fall ist (Roßteutscher 2009: 54). Eine Person wird also entweder in eine religiöse Organisation hineinsozialisiert oder entscheidet sich bewusst für die Beteiligung in einer solchen (Clark/Wilson 1961: 135 f., 146 f.).

\footnotetext{
${ }^{27}$ Des Weiteren existiert die entgegengesetzte (ebenfalls im US-amerikanischen Kontext) generierte These des ,avoiding politics“ (in USA): Unabhängig von einem religiösen Kontext sei freiwilliges Engagement häufig eingebunden in eine „Kultur der politischen Meidung“ (,culture of political avoidance“), um sich u. a. auf soziale, zwischenmenschliche Aspekte zu konzentrieren und auch um den Zusammenhalt der Gruppe nicht zu gefährden (Eliasoph 1998).
} 
Das soziale Vertrauen in die Gruppe und eine effektive Normensanktionierung werden, wie bereits erwähnt, ebenso durch auf Dauer gestellte zwischenmenschliche Bindungen ausgebildet: Durch den Aufenthalt in der religiösen Einrichtung, durch den Besuch von Gottesdiensten, durch die gemeinsame Ausübung von religiösen Praktiken kommen Personen in Kontakt mit anderen Mitgliedern und glaubensbasiertes Sozialkapital kann entstehen. Aufgrund der geteilten Werte, Normen und Erfahrungshorizonte entwickeln sich in religiösen Organisationen besonders enge Freundschaften und relativ dichte Netzwerkstrukturen (Olson 1989; Olson 1993: 42 f.; Becker/Dhingra 2001), die die Bereitschaft für gemeinschaftliche Zusammenarbeit und Engagement innerhalb der religiösen Gruppe, aber auch außerhalb der eigenen Gemeinde in der Gesamtgesellschaft verstärken können (Smidt 2003b: 15; Nemeth/Luidens 2003: 120; Jackson et al. 1995). Es sind die geteilten Werte, die in religiösen Organisationen den Zusammenhalt und die Identifizierung mit der Gruppe gewährleisten und einen zentralen Mechanismus für die Einbindung des Individuums in die Gemeinschaft darstellen. Hinzu kommt, dass religiöse Motive - wie der Wunsch, etwas Gutes zu tun und damit einem göttlichen Auftrag oder Willen zu entsprechen - die Bereitschaft, sich zu engagieren und zu kooperieren, erheblich intensivieren können (Smidt 2003c: 217 f.; Wald et al. 2005: 132; s. dazu auch 4.4.2).

Die durch die Wertehomogenität begünstigten Beziehungen (Welch et al. 2004: 318 f.; Cnaan et al. 2003: 28 f.; Kecskes/Wolf 1996: 15, 22, 25 f.) richten sich häufig auf die eigene religiöse Gruppe (Wuthnow 1999), deren Ziele und „Instandhaltungsaktivitäten“ (Campbell/Yonish 2003: 100, 102; s. auch Park/Smith 2000: 282 f.; Stolle/Rochon 1998). Das im religiösen Feld vielfach erbrachte Engagement kann demnach binnenorientiert sein und freiwilligem Engagement in nichtreligiösen Gruppen tendenziell entgegenstehen (Lam 2002: 415): „[W]e would predict that members of those religious traditions that have formed their collective identities through the construction of strong symbolic boundaries between the religious in-group and surrounding out-groups are likely to display diminished trust in neighbors, co-workers, and people in general" (Welch et al. 2004: 319; Uslaner 2002b: 239; Stolle 2002). Park und Smith (2000: 283) sprechen von einer ,,community insularity": einer gewissen Inselexistenz und Abgeschlossenheit religiöser Gemeinschaften (vgl. auch Paxton 2002: 270). Deren Kohäsion kann durch die Betonung exklusiver sozialer Distinktionskriterien, einer einzigartigen kollektiven Identität, durch heilsgeschichtliche Erwartungen wie auch Missionierungsanliegen weiter verstärkt werden. Exklusivistische Wahrheitsansprüche der eigenen religiösen Lehre und gruppeninterne soziale Abgrenzungen gegenüber der Gesellschaft 
können zu einem extremen Bonding im Sinne einer Selbstisolierung führen, die gemeinwohlorientierte Engagements und gesellschaftliche Integrationsprozesse unterbinden.

So kann es dazu kommen, dass religiöse Vereinigungen Gruppenegoismen, unsozialen Verhaltensweisen und Vorurteilen Vorschub leisten (Allport 1971: 444-456; Emerson/Smith 2000: 158; Laythe et al. 2002). Soziale Umgangsformen werden dann nur innerhalb der eigenen als vertrauenswürdig geltenden Gruppe realisiert (Maloney et al. 2008: 272) und erzeugen keine positiven externen Wirkungen. Die Gruppenidentifikation, die Verbindlichkeit der Gruppennormen und die Gruppenkohäsion können Coleman (1990: 318-320, 1988: 107 f.) zufolge durch die bereits erwähnte Geschlossenheit von Netzwerken (,closure of social networks") gewährleistet werden, da Pflichten und Erwartungen formuliert werden und bei Nichteinhaltung von Gruppennormen durch Mitglieder aufgrund der erhöhten sozialen Kontrolle mit Sanktionen gedroht werden kann (Finke et al. 2006: 624; Bekkers/Schuyt 2008: 76 f.). Je homogener eine Gruppe ist, desto größer ist die Wahrscheinlichkeit, dass sie sich schließen und Bonding-Sozialkapital ausbilden wird.

Die Stärkung der Mikrobindungen zwischen Individuen bei gleichzeitiger Unterbindung von Beziehungen zu anderen Gemeinschaften werden als Paradoxon der Bildung von Gruppen im Allgemeinen oder ,paradox of group loyalty“ (Emerson/Smith 2000: 158-160) in religiösen Gemeinschaften im Besonderen bezeichnet. Dabei kann die Abgrenzung nicht nur auf eine interne Gruppendynamik, sondern auf die intensive und andauernde Einbindung von Mitgliedern in die Vereinigung zurückzuführen sein, wenn diese mit „Leib und Seele“ viel Zeit in ihr Engagement investieren, in die Vereinigungsarbeit hineinwachsen und entsprechend auch seitens anderer Mitglieder oder Amtsträger mit Ansprüchen und Erwartungshaltungen konfrontiert werden (Horch 1983: 47, 50; Bekkers/Schuyt 2008: 76 f.). Für andere Bindungen und extraorganisationale Aktivitäten bleibt dann keine Zeit und Energie mehr übrig (Kanter 1968: 507 ff.; Brown 1969: 353 f.). Dieser Mechanismus der ,zeitlichen Segregation“ (Horch 1983: 47) kann bedeuten, dass die Vereinigung für eine Personen zu ihrem ausschließlichen Leben wird. Dies kann jedoch nur der Fall sein, wenn das betreffende Individuum auch persönliche Befriedigungen und Belohnungen wahrnimmt (Kanter 1968: 504). Diese können sich auf Interaktionen, gemeinsame Aktivitäten, die Gruppenkultur, Anerkennung, Prestige und Achtung beziehen (s. dazu auch 4.4.1). 
Die zeitliche Absorption kann bei rigiden religiösen Gruppen, Sekten und Eliten allerdings auch eine bewusst verfolgte Strategie darstellen, um den Wahrheitsanspruch und die Reinheit der eigenen religiösen Lehre gegen als bedrohlich oder feindlich wahrgenommenen Einflüssen von außen zu verteidigen (Kanter 1968; Etzioni 1975: 274 ff.; Roßteutscher 2009: 60). Von den Mitgliedern und Anhängern kann bewusst ein hoher Einsatz für die Gruppe gefordert werden, der all ihre Kräfte und Zeit absorbiert und den Mitgliedern folglich keinen Raum mehr lässt, um sich außerhalb der „bindenden“ und isolierenden Organisation zu engagieren oder anderweitig zu betätigen (Iannaccone 1994: 1197; Kelley 1995: 78 ff.; Wuthnow 1999). Je engmaschiger die organisationsinternen Beziehungen, je höher der individuelle Einsatz, die Inanspruchnahme der Mitglieder und die an sie gestellten Leistungsanforderungen ausfallen, desto stärker sind der Loyalitätsdruck gegenüber den Mitgliedern und die Abgrenzung nach außen (Finke et al. 2006: 624). Und: Je dogmatischer, rigider, fundamentalistischer eine religiöse Gruppe ist, desto eher wird sie die Einhaltung von Geund Verboten kontrollieren, sich das Vertrauen nur auf die eigene Gruppe beziehen und diese sich gegenüber der Umwelt abschotten. Extremfälle stellen die in allen Weltreligionen existierenden fundamentalistischen Strömungen dar, die autoritäre, intolerante Einstellungen und Vorurteile erzeugen, und denen es ,nicht um Menschengeschichte, sondern um Heilsgeschichte [geht], in der Kompromiß und Pluralismus nicht Tugend, sondern Verderbnis bedeuten" (Riesebrodt 2000: 90). Mit ihrem exklusiven Wahrheits- und Absolutheitsanspruch propagieren sie schwarz-weiße Welt- und Feindbilder (etwa: die Welt Gottes/des Guten - die Welt Satans/des Bösen; Marty/Appleby 1996: 39), die mit verabsolutierten Normen und Verhaltensweisen demokratischen Normen entgegenstehen, letztere gewaltsam bekämpfen und unterminieren sollen (Magen 2004: 33). ${ }^{28}$

\footnotetext{
${ }^{28}$ Damit ist verallgemeinert ein komplexes Phänomen angesprochen, für das es keine einfachen Erklärungen gibt und das an dieser Stelle nicht tiefergehender zu diskutieren ist (siehe dazu u. a. Marty/Appleby 1996; Almond et al. 2003; Kienzler 2007; Wippermann 2013; Tibi 1995, 2003). Es soll hier jedoch darauf hingewiesen werden, dass Motivationsquellen bei den sich religiös verortenden oder ihr Handeln im Nachhinein (pseudo-)religiös legitimierenden Individuen auszumachen sind, aber nicht per se bei den Religionen. Den Grad der Internalisierung religiöser Normen als Erklärungsfaktor für subjektives und kollektives Handeln heranzuziehen, reicht keinesfalls aus. Vielmehr sind sozioökonomische Bedingungen der Lebensumwelt, Ausgrenzungs- und Benachteiligungserfahrungen und weitere gesellschaftliche Rahmenbedingungen zu berücksichtigen. Ursachen sind vielschichtig, wirken kumulativ und sind wiederum nur kontextgebunden zu verstehen (s. dazu u. a. Heitmeyer et al. 1997; Uslucan 2016).
} 
Das den Religionen inhärente Paradox bzw. die Janusköpfigkeit ihrer Rollen liegt damit in ihren potenziellen doppelten Tendenzen in zwei Richtungen begründet: Diese umfassen vielfältige Ausprägungen auf einem Kontinuum mit jeweils zwei extremen Endpolen (Tab. 4.1).

Tab. 4.1 Bridging- und Bonding-Dimensionen religiöser Organisationen auf einem Kontinuum mit idealtypischen extremen Endpolen

\begin{tabular}{|c|c|c|c|c|}
\hline Dimension & Kon & inuum mit extremen Endp & & \\
\hline $\begin{array}{l}\text { Gottesbild } \\
\text { (Wahrheitsanspruch) }\end{array}$ & von & inklusivistisch & bis & exklusivistisch \\
\hline Werte und Normen & von & $\begin{array}{l}\text { prosozial (Nächstenliebe } \\
\text { etc.) }\end{array}$ & bis & $\begin{array}{l}\text { traditionalistisch, autoritär, } \\
\text { kompromisslos }\end{array}$ \\
\hline $\begin{array}{l}\text { Aufgeschlossenheit } \\
\text { gegenüber anderen } \\
\text { Überzeugungen/ } \\
\text { Handlungsweisen }\end{array}$ & von & tolerant & bis & intolerant \\
\hline Außenorientierung & von & $\begin{array}{l}\text { öffentlichem sozialen und } \\
\text { politischen Engagement }\end{array}$ & bis & $\begin{array}{l}\text { Rückzug, Abschottung, } \\
\text { sozialer Schließung }\end{array}$ \\
\hline $\begin{array}{l}\text { Haltung zur } \\
\text { Demokratie }\end{array}$ & von & Befürwortung & bis & Ablehnung \\
\hline Soziale Kontrolle & von & $\begin{array}{l}\text { effektiver Normensanktio- } \\
\text { nierung }\end{array}$ & bis & $\begin{array}{l}\text { Zwang } \\
\text { (Gewalt, Drohungen) }\end{array}$ \\
\hline
\end{tabular}

In dieser Arbeit sollen Informationen über die Generierung von Bonding- und Bridging-Sozialkapital in den bzw. durch die MSO gewonnen werden. In den Blick geraten damit zuvorderst die Ausrichtungen und Zielgruppen ihrer Aktivitäten, ihre Zielsetzungen, Selbstverständnisse, Mitgliedschaftsvoraussetzungen sowie ihr Kooperationsverhalten und damit das Ausmaß ihrer Öffnung nach außen.

Im Bezugsrahmen der in diesem Kapitel dargelegten Theoretisierungen zu (religiösem) Sozialkapital ist verdeutlicht worden, dass Bridging- und Bonding-Ausrichtungen von Vereinigungen mit dem Faktor Religion erklärt werden können. Darüber hinaus sind aber auch andere Organisationsmerkmale wie personelle und finanzielle Ressourcenausstattungen, die Mitgliederstruktur sowie Ausgrenzungserfahrungen und Negativ-Diskurse von Bedeutung. Spezifische 
Handlungsformen sind demnach mehrfach bedingt; sie treten in Kombination mit unterschiedlichen Organisationseigenschaften auf und werden nicht einfach in Entweder-Oder-Dimensionen einzuordnen sein.

Zur Überprüfung von Bonding- und Bridging-Ausrichtungen und relevanten Erklärungsfaktoren wird in dieser Arbeit daher bewusst eine offene Vorgehensweise mit unterschiedlichen Auswertungsverfahren gewählt (III. 7.2). Zunächst unabhängig von der Zuordnung zu religiösen Traditionen sind in einem ersten Schritt Bonding- und Bridging-Charakteristika zu identifizieren (III. 7.2.1). Im Rahmen der quantitativen, in Teilen aber auch qualitativ ausgerichteten Datenauswertung mit interpretativen Anteilen sollen auf Basis auszumachender Varianzen bzw. Muster u. a. Bridging- und Bonding-Typen von Organisationen identifiziert werden (III. 7.2.4). Bei diesen ist gesondert zu prüfen, welche Organisationsmerkmale innerhalb eines Typus jeweils sichtbar werden. Von Erkenntnisinteresse ist dann in einem zweiten Schritt, ob zwischen den unterschiedlichen Glaubensrichtungen hinsichtlich der Schaffung von religiösem Sozialkapital Differenzen festzustellen sind und ob einige religiöse Vereinigungen für die eine oder andere Ausprägungsform quasi als prädestiniert erscheinen.

Diese Auswertungsschritte werden schließlich flankiert und ergänzt von statistischen multivariaten Analyseverfahren. So wird v. a. mit multiplen Regressionsanalysen geprüft, ob zwischen spezifischen Merkmalen der MSO sowie Bondingund Bridging-Ausprägungen signifikante Zusammenhänge festzustellen sind (III. 7.2.5.4).

Im Rahmen der für die Analyse von bonding und bridging gewählten verschiedenen Auswertungsverfahren (III. 7.2) sollen folgende Fragen geklärt werden:

- Haben Binnenorientierung, fehlende Kontakte zu und fehlende Offenheit gegenüber der Umwelt mit Religion zu tun und lassen sich zwischen den Religionen Unterschiede aufdecken?

- Unter welchen Bedingungen bleiben die MSO unter sich?

- Lassen sich finanzielle Mängel, personelle Ressourcenausstattungen oder das Alter der Organisationen als Erklärungsfaktoren für auf die organisationale Binnenwelt gerichtete Performanzen identifizieren?

- Sind auszumachende Bonding-Tendenzen tatsächlich auf die theoretisch als ursächlich angenommene homogene Mitglieder- und Engagiertenstruktur zurückzuführen (Personengruppen, Konfessionszugehörigkeit, Herkunftsländer)?

- Inwieweit ist die Wahrnehmung von Fremdenfeindlichkeit, gesellschaftlichen Fehlplatzierungen und städtischer Nicht-Einbindung seitens der MSO zu registrieren und erklärt möglicherweise auf die eigene Gruppe konzentrierte Tätigkeiten? 


\subsection{Organisationssoziologische Kernelemente von Organisationen und die Eigenarten freiwilliger, religiöser und migrantischer Vereinigungen}

Die Organisationssoziologie befasst sich mit der theoretischen und empirischen Untersuchung von Organisationen als einer spezifischen Form von Sozialgebilden unterschiedlicher Typologie. Gemeint sind Unternehmen, Universitäten, Schulen, Parteien, des Weiteren Krankenhäuser und Kirchen sowie Verbände, Vereine und Nichtregierungsorganisationen. Solche Organisationen sind abzugrenzen von sozialen Netzwerken, sozialen Bewegungen und informellen Gruppen (Kühl 2014: 343; Tacke 2010: 341). Die Organisationssoziologie greift auf eine Vielfalt von Organisationstheorien und Begriffen zurïck. Ein Schwerpunkt der Analyse liegt dabei auf den Zielen und Strukturen der Organisationen sowie auf nach innen und außen wirkende und gerichtete Prozesse der Konsolidierung, Entwicklung und Veränderung. Damit unmittelbar verknüpft ist der Fokus auf spezifische Funktionsweisen, die durch die Art der Arbeitsteilung, ein bestimmtes Kooperationsverhalten und Abhängigkeitsverhältnisse gekennzeichnet sind. Dabei muss berücksichtigt werden, ob und inwiefern die sozialen, politischen und kulturellen Umweltbedingungen die Organisationen in ihrem Handeln eher einengen und einschränken oder vielmehr Möglichkeiten der Entfaltung bereithalten (Tacke 2010: 341).

Bei der Analyse von Organisationen gilt es in der Organisationssoziologie als unverzichtbar, zunächst Basis- bzw. Kernelemente von Organisationen zu betrachten, die in der Summe das Fundament für die tatsächlichen Vorgänge in einer Organisation bzw. für das organisationale Handeln darstellen. In Anlehnung an Richard W. Scott und Gerald F. Davis (2007) lassen sich vier interne Basiselemente einer Organisation identifizieren: 1.) die Mitglieder, 2.) die Ziele oder Zwecke der Organisation, 3.) ihre Ressourcenausstattung sowie 4.) die formale Struktur (interner Aufbau, offizielle Abläufe etc.). Hinzu kommt 5.) die Charakterisierung von Ebenen und Dimensionen der Umwelt als ein externes Element (vgl. auch Preisendörfer 2016: 59-82).

Die fünf Komponenten sind miteinander verzahnt und lassen sich in unterschiedlichen Ausprägungen in allen Organisationen finden. Sie können auch für den Organisationstyp des Vereins Geltung beanspruchen, wobei hier - im Vergleich zu anderen Organisationsformen - das Komplexitätsniveau insbesondere hinsichtlich der formalen Organisationsstruktur wesentlich niedriger ausfällt. Auch sind die Organisationskomponenten inhaltlich nicht immer trennscharf zu fassen; anhand nachfolgender Erläuterungen werden vielmehr fließende Übergänge deutlich. So sind Organisationsziele nicht isoliert von Mitgliedern und 
deren persönlichen Bedürfnissen zu denken; die Mitglieder werden in ihrem Verhalten von der formalen Struktur gelenkt und prägen - umgekehrt - wiederum die organisationale Ordnung und das Binnenleben. Zugleich stellen sie eine wichtige Ressource der Organisation dar und beeinflussen deren Verhältnis zur Umwelt.

\section{Vereine als organisationssoziologischer Sondertypus}

Die benannten Kernelemente basieren nicht auf einer einzigen Theorie, sondern sie sind je nach organisationstheoretischer Denkschule und soziologischem Modell unterschiedlich konturiert bzw. argumentativ verankert. Dabei ist zu berücksichtigen, dass sich bei der Thematisierung der einzelnen Komponenten zumeist auf große kommerzielle, gewinnorientierte Arbeitsorganisationen mit einer bezahlten Belegschaft konzentriert wird und Spezifika freiwilliger, religiöser sowie migrantischer Vereinigungen entsprechend unberücksichtigt bleiben. In diesem Zusammenhang ist grundsätzlich anzumerken, dass Vereine, religiöse Organisationen (jenseits von Kirchen) und damit auch MSO aufgrund ihrer besonderen bzw. ,Un-Typik“ in den Organisationstheorien vernachlässigte Organisationsformen darstellen (MüllerJentsch 2008; Karstein 2013; Horch 1983; Petzke/Tyrell 2012; Kehrer 1988: 12 ff.). Vor diesem Hintergrund beschreibt Karin Weiss (2013: 22) MSO als „eine Organisationsform entlang der Herkunft, die nicht in die gängigen Muster der Organisationssoziologie passt." Hingegen haben Sportvereine immerschon eine hohes fachwissenschaftliches, auch organisationssoziologisches Interesse auf sich gezogen. ${ }^{29}$ Mit Klaus Heinemann und Heinz-Dieter Horch (1981: 124 f.) lassen sich fünf Merkmale von Sportvereinen bestimmen, die allgemein auch für die differentia specifica des Vereins Geltung beanspruchen: die Freiwilligkeit der Mitgliedschaft, die freiwillig-unbezahlte bzw. ehrenamtliche Mitarbeit, die Orientierung an den Mitgliederinteressen, demokratische Entscheidungsstrukturen sowie die Unabhängigkeit vom Staat. In der Logik der Zivilgesellschaft (3.1) ist „eine nicht-wirtschaftliche (non profit), gemeinwohlbezogene, solidarische und gesellige Zwecksetzung“ (Jütting 2008: 133) zu ergänzen.

\section{Pluralität abstrakter Theorien}

Diese durch die Forschungssituation bedingten „blinden Flecken“ sowie die auf einem hohen Abstraktionsniveau angesiedelte Theoriepluralität, die ein One-fitsall-Konzept nicht erwarten lässt, erklärt einen gewissen Eklektizismus, mit der in

\footnotetext{
${ }^{29}$ Siehe aus einer Vielzahl an Literatur z. B. Heinemann/Horch 1981, 1988; Digel 1988; Zimmer 2005; Schimank 2005; Thiel/Mayer 2009.
} 
den folgenden Kapiteln die fünf Basiselemente diskursiv erarbeitet werden: Organisationsziele (4.3.1), Ressourcenausstattung (4.3.2), Mitglieder (4.3.3), Dimensionen der formalen Organisationsstruktur (4.3.4) sowie die Organisationsumwelt (4.3.5). Angesichts der Ausgangslage werden zentrale organisationstheoretische Prämissen dargelegt und zugleich hinsichtlich ihrer Relevanz für und Anwendbarkeit auf den Forschungsgegenstand kritisch reflektiert. Es zeigt sich, wie freiwillige und religiöse Vereinigungen in den einschlägigen Organisationstheorien häufig weitgehend unberücksichtigt bleiben. Daher findet in den Kapiteln auch eine Kontrastierung bzw. ein Abgleich von in Arbeits- und Freiwilligenorganisationen vorgefundenen Verhältnissen und Logiken statt. Bezug genommen wird dabei auch auf konkrete Regelungen des im Bürgerschaftlichen Gesetzbuch (BGB) geregelten Vereinsrechts ( $§ 21$ bis 79 BGB), die die formale und v. a. formalrechtliche Struktur der Vereine wesentlich bestimmen.

Vor diesem Hintergrund ist das Funktionsgefüge oben benannter komplexer Faktoren in unterschiedliche theoretische Horizonte einzubetten bzw. aus verschiedenen theoretischen Blickwinkeln zu betrachten. Dies bedeutet, dass neben dem organisationstheoretischen Fokus auf Ziele und Funktionsweisen von Organisationen auch Aspekte subjektiven Handelns sowie individuelle Verhaltensweisen in Gruppen bzw. in Organisationen (engl. organizational behavior; Weibler 2007) berücksichtigt werden müssen. Dabei stellen sowohl extra- als auch intraorganisationale Kontexte den Referenzrahmen für das menschliche Verhalten dar. Ein relevanter Aspekt ist, wie das Handeln von Mitgliedern durch strukturelle, prozessuale und soziale Bedingungen innerhalb der Organisationen beeinflusst und gesteuert werden kann. Die verschiedenen Rollen als Individuen, Gruppen- und Organisationsmitglieder lassen sich dabei nicht trennscharf voneinander abgrenzen, sondern gehen ineinander über. Denn es ist davon auszugehen, dass individuelle Interessen und Ziele in den Organisationskontext eingebracht werden und dortiges Handeln sowohl im Interesse des Subjekts als auch der Gruppe bzw. Organisation erfolgt.

In Hinblick auf die Ausgestaltung und Gewährleistung organisationsinterner Abläufe geraten verhaltenssteuernde Wirkungen von sozialen Regeln (Normen), wechselseitigen Verhaltenserwartungen bzw. Erwartungssicherheiten, Kommunikations- und soziale Interaktionsprozesse in den Blick, die in der Summe kollektive Verbindlichkeiten und kollektive Handlungsfähigkeit herstellen. Die theoretischen Horizonte dienen in den folgenden Kapiteln damit auch der Erläuterung von wichtigen ordnungsstiftenden Regelungen und gruppensoziologischen Prozessen innerhalb von Organisationen (4.3.4). Darüber hinaus werden auch Ergebnisse empirischer Studien eingebunden, beispielsweise bei der Thematisierung einer 
immer geltenden Ressourcenabhängigkeit der Engagementbeteiligung von Individuen (4.3.3.1), die letztlich auch beeinflusst, ob sich eine Person für eine freiwillige Aufgabenübernahme innerhalb einer Organisation entscheidet oder dieses nicht tut.

Mit der Darlegung der mit den einzelnen Organisationskomponenten in der einschlägigen Literatur verknüpften Denkansätzen wird ein Blick ,,hinter die Kulissen“ von Organisationen geworfen. Eine Inbezugsetzung von Theorie und Empirie kann - wie in der Einleitung (I. 1) bereits herausgestellt wurde - nur in Teilen erfolgen. Angesichts der theoretischen Ausführungen sind insofern Bruchstellen zwischen Theorie und Empirie zu konstatieren. Den Theoriekapiteln kommt somit eine dreifache Funktion und damit ein eigenständiger Stellenwert zu: Sie dienen 1.) der Konturierung der zentralen das organisationale Handeln charakterisierenden Größen, 2.) der Einordnung des Forschungsgegenstands in den Stand der Theorie sowie 3.) der Operationalisierung der Forschungsfragen (III. 6.1). Neben der Darlegung der Basiselemente erfolgt die Formulierung von theoretisch verorteten und empirisch zu überprüfenden Hypothesen.

\section{Vereine und Verbände als genuine Mitgliederorganisationen}

Angesichts ihrer Relevanz für den Forschungsgegenstand soll vor der Explikation der Basiselemente in den folgenden Kapiteln an dieser Stelle die Aufmerksamkeit auf erwähnenswerte Charakteristika der zwei genuinen Mitgliederorganisationen des Vereins und des Verbandes gelenkt werden. Die zwei Organisationstypen stellen Rahmen für unterschiedliche Formen und Ausdrucksweisen freiwilliger Engagements dar. In diesem Zusammenhang ist darauf hinzuweisen, dass sich die muslimischen Selbstorganisationen nach Art. 140 GG i.V.m. Art. 137 Abs. 4 WRV ${ }^{30}$ dominant in Vereinsform ( $§ 21 \mathrm{BGB}$ ) organisiert haben, um die Rechtsfähigkeit einer juristischen Person zu erlangen und entsprechend handeln zu können, da sie als Körperschaften des öffentlichen Rechts nicht anerkannt werden (Lemmen 2002: 27).

Insbesondere der Verein stellt (gemäß $\S 21$ bis 79 BGB) sowohl eine flexible Rechts- als auch demokratische Organisationsform dar (Zimmer 2011: 453). Das Recht der Vereinsgründung ist in Deutschland im Grundgesetz (Art. 9 GG) verankert und mit der Versammlungsfreiheit und freien Meinungsäußerung (Art. 8 u. Art. 5 GG) assoziiert. Darüber hinaus ist es auch durch den Artikel 20 der Allgemeinen Erklärung der Menschenrechte von 1948 geschützt. Trotz gemeinsamer Ursprünge wird im deutschsprachigen Raum stärker zwischen Vereinen und Verbänden differenziert (Heinze 2011), was insbesondere auf die Ausführungen von Max Weber

\footnotetext{
30 „Religionsgesellschaften erwerben die Rechtsfähigkeit nach den allgemeinen Vorschriften des bürgerlichen Rechts."
} 
zurückzuführen ist. Weber verstand unter Vereinen ,,alles das, was zwischen den politisch organisierten oder anerkannten Gewalten - Staat, Gemeinde und offizielle Kirche - auf der einen Seite und der naturgewachsenen Gemeinschaft der Familie auf der anderen Seite in der Mitte liegt" (Weber 1911: 52; vgl. auch Weber 1988/[1924]: 441 f.). Den Verband definierte Weber in erster Linie als Herrschaftsverband, in dem die Einhaltung von vereinbarten oder oktroyierten Regeln durch die Gefolgschaft auch durch eine Führungsperson erzwungen werden kann (Weber 1985/[1922]: 26).

Die Organisationsform des Verbandes ist aufgrund seines Kennzeichens der Interessenvertretung der Mitglieder im politischen Willensbildungs- und Entscheidungsprozess in erster Linie zum Forschungsgegenstand der Politikwissenschaft geworden. Der Verein als „Basiselement der Demokratie“ (Zimmer 1996) kann zwar auch politische Belange vertreten und politische Sozialisation vermitteln, widmet sich jedoch in erster Linie der gemeinschaftlichen Ausübung von sozialkulturellen Interessen in unterschiedlichen gesellschaftlichen Bereichen und wird daher eher in der Soziologie erforscht (Zimmer 2007; Schmidt 2010: 827, 830; Heinze 2011: $465 \mathrm{ff}$.$) .$

\section{Organisationssoziologische Paradigmen nach Richard W. Scott und Gerald F. Davis}

Im Folgenden sollen zunächst komprimiert die drei grundlegenden von Scott und Davis (2007) in die Organisationssoziologie eingeführten Organisationskonzeptionen bzw. -paradigmen mit ihren inhaltlichen Merkmalen skizziert werden. Die Kenntnis dieser drei unterschiedlichen konzeptionellen Dächer gibt Orientierung hinsichtlich des Verständnisses und der Einordnung verschiedener Organisationstheorien, auf deren Basis die Kernelemente von Organisationen erläutert werden.

Scott und Davis unterscheiden bei der Analyse von Organisationen zwischen Organisationen als 1.) rationale, 2.) natürliche bzw. soziale und 3.) offene Systeme (Scott/Davis 2007: 35-106; s. auch Preisendörfer 2016: 103-175). Wenngleich die Einteilung nicht trennscharf ist, lassen sich die verschiedenen Organisationstheorien mit ihren zentralen Prämissen zumeist recht eindeutig einer der Systemperspektiven zuordnen (vgl. Tab. 4.2).

In der Perspektive von Organisationen als rationale Systeme stellen Organisationen rational geplante und steuerbare Kollektive dar, die auf die effiziente Realisierung spezifischer Ziele als Richtschnur des Organisationshandelns ausgerichtet sind und die Gestaltungsparameter aufweisen, die auf relativ stark formalisierten normativen Strukturen basieren. Diese Strukturen betreffen die Formalisierung von Regeln und Rollen und die Leistungen der Mitarbeiter, die 
wiederum als Instrumente für die Erreichung präferenz- und nutzenorientierter Ziele verstanden werden. Dem Management wird eine zentrale Steuerungsfunktion zugeschrieben.

Bei der Betrachtung von Organisationen als natürliche bzw. soziale Systeme geraten die Teilnehmer als lebendige Menschen mit unterschiedlichen persönlichen Interessen und Ideen sowie das soziale Interaktionsgeschehen in Organisationen in den Blick. Die verhaltenswissenschaftliche Entscheidungstheorie gilt als Ursprung und Interpretament für diese Sichtweise (Preisendörfer 2016: 127). An technischen Kriterien der Rationalität wird Kritik geübt, da bei dieser nichtrationale Aspekte des sozialen Verhaltens nicht zum Zuge kommen. In Organisationen sei häufig eine Diskrepanz festzustellen zwischen den erklärten Zielen und normativen Vorgaben einerseits und dem faktischen Geschehen bzw. tatsächlich - auch nichtrational - verfolgten Zielen andererseits. Häufig verliefen Dinge anders als geplant; zudem hätten Organisationen nicht nur Output-Ziele, sondern auch Ziele der Instandhaltung und der Mitarbeiterakquise zu verfolgen. Bei letzterer geht es auch um Beziehungsarbeit, etwa um die Frage, wer zu einem Team passt und wer nicht, was allein durch rationale Kriterien nicht zu beantworten ist. Während die rationale Systemperspektive die Bedeutung der normativen Organisationsstruktur betont, unterstreicht die natürliche Systemdefinition die Relevanz von individuellen Interessen, Rücksichten und Fähigkeiten.

Die Sichtweise auf Organisationen als offene Systeme entwickelte sich später als die rationale und natürliche Systemperspektive. Das Paradigma unterstreicht die Komplexität und Wandelbarkeit gegenüber der Beständigkeit von festen Strukturen sowie die Interdependenzen und Bindungen zwischen Organisationen und ihrer Umwelt, in die sie eingebettet und von denen sie abhängig sind. Für Lebensfähigkeit und Bestand des offenen Systems wird der Austausch mit der Umwelt geradezu als zentraler Faktor betrachtet, da die Umwelt als unerlässliche Quelle für Informationen, Ressourcen und Legitimität fungiert (Scott/Davis 2007: 106). Für ein Agieren und Arbeiten im offenen System spielen kulturelle, kognitive und institutionelle Dimensionen des sozialen Lebens eine bedeutende Rolle, und zwar weniger als gegebene Struktur, denn als lebendiger Prozess, insofern das offene System, will es seiner Offenheit gerecht werden, ständige Kommunikation mit Beteiligten außerhalb der Organisation voraussetzt bzw. erforderlich macht.

Im Zusammenhang mit dem Umweltbezug ist die Frage der Grenzziehungen von Organisationen zentral: Wie viel Umweltbezug ist erforderlich, wie viel riskant? Wenn in das Organisationsgeschehen viele Individuen und Gruppen involviert sind, werden Kontrolle und Koordination zweifellos schwieriger, gleichzeitig kann sich eine gewisse Dynamik entwickeln, die handhabbare Balancen einschließt. Die Berücksichtigung von Organisation-Umwelt-Beziehungen gilt für das Verständnis 
von organisationalem Handeln als Conditio sine qua non. Dabei wird jedoch häufig die Beeinflussung der Organisationen durch ihre Umwelt fokussiert und nicht andersherum (Preisendörfer 2016: 150). Der Versuch, umgekehrt die Umwelt zu beeinflussen, wird alle Ressourcen, die einer Organisation zur Verfügung stehen, mobilisieren - rationale, natürliche und soziale.

Die Merkmale, nach denen sich Organisationen zuvorderst unterscheiden, sind durchaus kombinierbar, und unter dem möglichen Druck der Verhältnisse (z. B. Bedeutungsverlust, Reaktionen auf unerwartete Ereignisse, Kampagnen der Aufklärung) auch zu Synthese gezwungen. Mögen die Organisationstypen auf analytischer Ebene unterscheidbar sein - auf praktischer Ebene - sind sie es nicht. Insofern können sich die drei beschriebenen Perspektiven durchaus kombinieren, was definitive Zuordnungen schwierig macht und sie bisweilen schwammig erscheinen lässt. Wie bereits konstatiert, kann dennoch eine Kategorisierung auf Basis dominanter theoretischer Foki erfolgen. Darüber hinaus kann der jeweilige Zeitraum, in der eine Theorie entstanden ist, für die Klassifizierung herangezogen werden (ebd.: 12; Tab. 4.2).

In den folgenden Kapiteln werden nun forschungsrelevante Dimensionen der bereits genannten Kernelemente Organisationsziele (4.3.1), Ressourcenausstattung (4.3.2), Mitglieder (4.3.3), Dimensionen der formalen Organisationsstruktur (4.3.4) sowie Ebenen und Dimensionen der Organisationsumwelt (4.3.5) erläutert und organisationstheoretisch substantiiert. Dieses ist auch relevant für die Operationalisierung der Forschungsfragen und die Konturierung der zentralen das Organisationshandeln beeinflussenden Parameter (III. 6.1).

\subsubsection{Organisationsziele}

Die meisten Definitionen des Organisationsbegriffs rekurrieren auf das Merkmal der Ziel- oder Zweckorientierung ${ }^{31}$ von Organisationen. So definiert Amitai Etzioni Organisationen beispielsweise als ,soziale Einheiten, die mit dem Zweck errichtet wurden, spezifische Ziele zu erreichen“ (Etzioni 1967: 12; vgl. auch Sills 1957: 11; Blau/Scott 1962: 5; Heintz 1962: 173 ff.; Mayntz 1963: 18 f., 36, 58 ff.; Parsons 1960: 63; Barnard 1970: 81 ff.; Scott 1986: 31; Luhmann 1991/[1973]: 55 ff.; Aldrich/Ruef 2006: 4 f.; Abraham/Büschges 2009: 58). Hinter den zweckbezogenen Begriffsbestimmungen steht die Annahme, dass die Kenntnis der Ziele für das Verständnis der Vorgänge in einer Organisation und ihres Handelns wichtig ist.

${ }^{31}$ Die Begriffe „Ziele“ und „Zwecke“ werden im Folgenden synonym verwendet. 
Tab. 4.2 Organisationskonzeptionen und -theorien - Ein komprimierter Überblick

\begin{tabular}{|c|c|c|c|}
\hline \multirow[t]{2}{*}{ Zeitdimension } & \multicolumn{3}{|c|}{ Organisationskonzeption } \\
\hline & Rationales System & $\begin{array}{l}\text { Natürliches/ } \\
\text { soziales System }\end{array}$ & Offenes System \\
\hline $\begin{array}{l}\text { Historische Ansätze } \\
\text { (vor dem 2. Weltkrieg) }\end{array}$ & $\begin{array}{l}\text { - Bürokratieansatz } \\
\text { (Max Weber) } \\
\text { - Wissenschaftliche } \\
\text { Betriebsführung } \\
\text { (Frederick Taylor) }\end{array}$ & $\begin{array}{l}\text { Human-Relations- } \\
\text { Schule } \\
\text { (Fritz Jules } \\
\text { Roethlisberger, } \\
\text { Elton Mayo) }\end{array}$ & \\
\hline $\begin{array}{l}\text { "Sturm \& Drang“- } \\
\text { Ansätze/Boomphase } \\
\text { der Organisations- } \\
\text { forschung nach } \\
\text { 2. Weltkrieg } \\
\text { (1960er/1970er Jahre) }\end{array}$ & & $\begin{array}{l}\text { Verhaltenswissen- } \\
\text { schaftliche Entschei- } \\
\text { dungstheorie }^{3}\end{array}$ & $\begin{array}{l}\text { Kontingenztheorie/ } \\
\text { "situativer Ansatz“6 }\end{array}$ \\
\hline $\begin{array}{l}\text { Neuere Ansätze } \\
\text { (ab 1980er Jahre) }\end{array}$ & $\begin{array}{l}\text { - Institutionenöko- } \\
\text { nomischer Ansatz: } \\
\text { Transaktionskosten- } \\
\text { ansatz }{ }^{1} \\
\text { - Agency-Theorie }{ }^{2}\end{array}$ & $\begin{array}{l}\text { - } \text { (Mikro-)Politischer }^{\text {Ansatz }}{ }^{4} \\
\text { - Systemtheorie }\end{array}$ & $\begin{array}{l}\text { - Organisations- } \\
\text { Ökologie }{ }^{7} \\
\text { - Ressourcen- } \\
\text { Abhängigkeitsansatz } \\
\text { (Jeffrey Pfeffer, } \\
\text { Gerald R. Salancik) } \\
\text { - Soziologischer } \\
\text { Neoinstitutio- } \\
\text { nalismus }^{8}\end{array}$ \\
\hline
\end{tabular}

Zusammenstellung in Anlehnung an Preisendörfer 2016: 13; Scott/Davis 2007: 112. Fettsetzung von in dieser Arbeit fokussierten Theorien.

${ }^{1}$ Ronald Coase, Oliver Williamsen, Mark Granovetter

2 Armen Alchian, Harold Demsetz, Eugene Fama, Michael Jensen, William Meckling, Stephan Ross

${ }^{3}$ Chester Irving Barnard, Herbert Alexander Simon, James G. March, Richard Michael Cyert, Johan Peder Olsen

${ }^{4}$ Erhard Friedberg, Michel Crozier, Oswald Neuberger

5 Niklas Luhmann (Daniel Katz, Robert Louis Kahn 1960er/1970er Jahre)

6 Drei Forschungsgruppen 1.) um Peter Blau an der University of Chicago; 2.) die Aston-Gruppe um Derek Pugh an der Aston University/ Birmingham, 3.) um Alfred Kieser an der Universität Mannheim

${ }^{7}$ Michael Hannan, John Freeman, Glenn Carroll

8 John Meyer, Brian Rowan, Paul DiMaggio, Walter Powell, Lynne Zucker, Richard Scott

Im Allgemeinen werden den Zielen einer Organisation in der Organisationsforschung unterschiedliche Funktionen zugeordnet: 1.) die Motivation von Mitgliedern, Mitarbeitern und Engagierten, indem ihnen über die Ziele verdeutlicht wird, ,was die Organisation erreichen will, und damit auch für jeden Einzelnen Hinweise liefer[t], was er oder sie dazu beitragen kann" (Preisendörfer 2016: 65). Die klare Festlegung von Zielen ermöglicht es 2.), den (Miss-)Erfolg einer Organisation einzuschätzen bzw. eine Erfolgskontrolle vorzunehmen, 3.) 
als Maßstab für die Ausrichtung von Entscheidungen zu fungieren sowie 4.) die Organisation gegenüber ihrer Umwelt zu legitimieren bzw. die Umwelt von ihren Zielen zu überzeugen, um Ressourcen zu gewinnen (ebd.).

\section{Organisationsziele im Blickwinkel der drei organisationssoziologischen Para- digmen}

Ziele bzw. Zwecke einer Organisation lassen sich zunächst in der Logik der drei skizzierten Paradigmen nach Scott und Davis beleuchten. Denn deren Charakteristika - rationale, natürliche bzw. soziale sowie offene - liefern eine erste Typologie, mit der Organisationsziele in ihren Bedeutungen modellhaft näher konturiert werden können.

Aus der rationalen Systemperspektive, die in der Organisationsforschung u. a. von Managern vertreten wird, sind Organisationen ,Kollektive, die auf die Verfolgung relativ spezifischer Ziele ausgerichtet sind und die relativ stark formalisierte soziale Strukturen aufweisen“ (Scott/Davis 2007: 29; Übers.: d. Verf.). Demnach soll hier nichts dem Zufall überlassen werden, Kontingenz ist möglichst auszuschalten. Kommunikation ist notwendig, um zielgerichtete Informationsflüsse als zweckmäßig eingesetzte Mittel zu befördern. Die Ausrichtung auf ökonomischen Erfolg erfordert explizite, klar definierte Ziele und einen kalkulierten Umgang mit Ressourcen. Auf die effiziente Realisierung von Zielen ausgerichtet zu sein, impliziert, dass adäquate Entscheidungen getroffen werden müssen, die die Aktivitäten und Interaktionen der Beteiligten koordinieren und die Organisation strukturieren (ebd.: 36). Insgesamt verlangt das rationale System eine nicht zu jeder Zeit unbedingt konforme, aber doch systemtreue, im Ganzen funktionalistische Ausrichtung, die Denken und Handeln einem eindeutigen Zweck unterordnet. Häufig verwendete Begriffe lauten entsprechend: Information, Wissen, Effizienz, Optimierung, Legitimierung, Strategie, Taktik, Koordination, (Verhaltens-)Regeln, Anweisungen, Autorität, Kontrolle. Sie verweisen auf die den Individuen hinsichtlich ihrer eigenen Entscheidungen und Handlungen in Organisationen auferlegten kognitiven und motivationalen Einschränkungen, insofern die Akteure sich dem Rationalisierungszwang unterordnen (ebd.; Simon 1981: 273). So erscheint es sehr nachvollziehbar, dass gewinnorientierte Unternehmen (als koordinierte Akteure) spezifische Output-Ziele festlegen und entsprechende Strategien verfolgen, um auf dem Markt wettbewerbs- und konkurrenzfähig zu bleiben.

Der natürlichen Systemperspektive zufolge lässt sich eine Organisation nicht allein entlang technisch-rationaler Kriterien lenken. Vielmehr sind auch nichtrationale Aspekte des sozialen Verhaltens und der verschiedenen Interessen der Mitglieder, die diese in die Organisation einbringen (wollen), zu berücksichtigen. 
Rationalität ist dabei zweifellos ebenfalls ein Kriterium, über das natürliche, sich sozial verpflichtende Personen verfügen müssen, allerdings nicht im Sinne einer funktionalistischen Ausrichtung ihres Handelns. Angesichts der Komplexität der Anliegen sozialer Akteure fungiert Rationalität nicht als generelles Strategem, sondern als Mittel für einen übergeordneten Zweck. Will ein Verein z. B. Hilfsleistungen für Migranten anbieten, so ist die Frage, wie man am besten helfen kann, sicherlich ein Feld rationaler Überlegung - dadurch etwa, dass man rational zu unterscheiden versteht zwischen sinnvoller und nicht sinnvoller Hilfe und genau überlegt, wem überhaupt welche Hilfe in erster Linie zukommen soll. Die natürliche Systemperspektive erweist sich insofern als natürlich und sozial, als dass das, was ,effizient“ sein könnte, nicht von vornherein feststeht, sondern Gegenstand von Diskussion und Aushandlung ist. Rationalität folgt hier keiner mechanischen Zuordnung von Mittel und Zweck, sondern ist bestenfalls Ausdruck umsichtiger Überlegungen und Abwägungen von Vernunftgründen.

Während bei der rationalen Systemperspektive ein rationales System - als übergeordneter Gesichtspunkt - erwünscht und erforderlich ist, haben Natürlichkeit und Sozialität eine andere Konsequenz: Anders als beim rationalen Systemzwang müssen sie innerorganisational immer wieder neu ausgehandelt werden, wobei die Mitglieder die Besonderheit des Sozialen festlegen. Dadurch werden verfolgte Ziele wie auch die Mittel, um sie zu realisieren, bei einer natürlichen und sozialen Organisation diffuser und komplexer; sie können von Aktion zu Aktion variieren und sind modifizierbar. Daher wird häufig von einer Diskrepanz ausgegangen zwischen den ursprünglich vereinbarten, offiziell proklamierten oder verschriftlichten Zielen und den tatsächlichen, im operativen Organisationsgeschehen relevanten Zwecken, die die Aktivitäten der Beteiligten anleiten (Scott/Davis 2007: 30, 60).

Die offene Systemperspektive geht noch einen Schritt weiter. Die Prozesshaftigkeit der Arbeit innerhalb und mit der Organisation wird als Konsequenz der Komplexität des Geschehens und der Wandelbarkeit der Situationen betrachtet. Die einzelnen Komponenten der Organisationsstruktur sind nur lose miteinander verbunden. Die Umwelt ist prinzipiell unberechenbar und unzuverlässig und mit festen Strukturen entsprechend nicht zu bewältigen. Fortdauernd müssen neue Informationen beschafft und bearbeitet werden. Die Frage, wie und womit man auf Veränderungen der Umwelt reagiert, impliziert die zentrale Frage nach der Legitimität des Handelns. Mehrere involvierte Personen treffen Entscheidungen und versuchen das Handeln in der Organisation anzuleiten, wodurch sich Koordination und Kontrolle schwieriger gestalten. Aufgrund personeller Fluktuation, die aus eher (un-)attraktiven Anreizen, die das offene System aussendet, resultieren können, geraten einst gesetzte handlungsanleitende Zweckorientierungen in den 
Hintergrund; sie gelten vielmehr nur für eine bestimmte Zeit und sind wandelbar (ebd.: 106).

Aufgrund seiner geringen Berechenbarkeit fällt eine Beurteilung des offenen Systems eher pessimistisch aus. Tatsächlich dürfte sich die Gründung und Aufrechterhaltung einer Organisation schwierig gestalten, wenn Ziele zwar formuliert, diese aber auch wieder infrage gestellt und abhängig gemacht werden von Veränderungen in der Umwelt. Wenn im offenen System gefragt wird, ob das, was man sich vor Monaten vorgenommen hat, jetzt noch Sinn macht, kann dies einen gewissen Reiz ausmachen. Jedoch bleiben Absichten und Vorhaben sowie Sinn und Ausrichtungen des organisationalen Handelns - zumindest eine Zeit lang - im Unklaren und Entscheidungen, z. B. bei wichtigen zu lösenden Problemen, werden dem Zufalls-, Improvisations- oder Mehrdeutigkeitsprinzip überlassen (Scott/Davis 2007: 219).

\section{Organisationsziele als entscheidungs- und handlungsanleitende Größen}

Chester Barnard (1970: 81) konstatiert in diesem Zusammenhang, dass Kooperationsbereitschaft im Rahmen von Zusammenschlüssen mehr sei ,,als ein unbestimmtes Gefühl oder der vage Wunsch nach Verbindung mit anderen“ und dass sie „sich nicht ohne ein Ziel für die Zusammenarbeit entwickeln [kann]. Ohne ein solches Ziel weiß niemand, welche Leistungen von den einzelnen verlangt werden müssen und welche Befriedigungen ihnen geboten werden können." Nicht nur die Mitglieder, sondern auch Akteure aus der Umwelt dürften von einer ziel- bzw. zwecklosen Organisation irritiert sein und der Organisation damit keine für ihre Existenz notwendige Legitimität zugestehen.

Allerdings wird das dominante ,Ziel-Paradigma“ (Preisendörfer 2016: 67) von einigen Organisationswissenschaftlern auch skeptisch betrachtet und als überbewertet kritisiert. So wird die Notwendigkeit der Kenntnis und die Betonung des Stellenwerts von Organisationszielen als die zentralen Entscheidungs- und Handlungsorientierungen steuernden Parameter auch von Niklas Luhmann als Vertreter der systemtheoretischen Position angezweifelt. Luhmann zufolge erfüllen Zwecksetzungen die wichtige Funktion der Komplexitätsreduktion und Unsicherheitsabsorption. Sie stellen die Grundlage für genau bestimmbare, auch modifizierbare Entscheidungsprämissen und einen Orientierungspunkt für die Binnendifferenzierung der Organisation dar (Luhmann 1964: 73 ff.; Luhmann 1990/[1973]: 179, 190 ff., 341 f.). Allerdings sei es ,weder möglich noch sinnvoll, alles Handeln in organisierten Systemen aus dem Organisationszweck abzuleiten“ (Luhmann 1964: 32). Die Zweckorientierung allein sei nicht instruktiv und stoße an ihre Grenzen, da sie allein den Systembestand nicht sichern könne (Luhmann 1991/[1973]: 191, s. auch 342). Daher plädiert Luhmann dafür, den Zweckbegriff „,zu entthronen“ und 
ihn, relativiert, unter dem Gesichtspunkt des Systembestandes in eine umfassendere Systemtheorie einzufügen (ebd.: 86, 191, 202).

\section{Zum Verhältnis von Organisationszielen und Mitgliederinteressen}

Andere Organisationswissenschaftler stellen die Frage nach den Organisationzielen noch grundsätzlicher. Erkenntniskritisch wird gefragt, was unter einem Organisationsziel eigentlich zu verstehen sei und wie es zustande kommt. Angenommen wird, dass Ziele von bestimmte Interessen verfolgenden Individuen gesetzt werden (Cyert/March 1963: 25 ff.). Mit der Perspektive auf Individuen und der Konzeption der Organisation als zielgerichteter Akteur bestehe jedoch die Gefahr einer reduktionistischen Verdinglichung (,Reifikation“) und „Personifizierung von Organisationen“ (Preisendörfer 2016: 67; Simon 1981: 273; Scott/Davis 2007: 186), insofern als das Agieren von Zusammenschlüssen mit der Logik des Handelns individueller Akteure tendenziell gleichgesetzt wird (Luhmann 1991/[1973]: 7 ff.; Vanberg 1983).

Herbert Simon (1981) unterstreicht in diesem Zusammenhang die Notwendigkeit einer Unterscheidung zwischen individuellen Motiven der Mitglieder, der Organisation beizutreten und sich dort einzubringen einerseits, sowie den Organisationszielen, die teilweise kollektive Interessen widerspiegeln und die konkrete Entscheidungen der Mitglieder veranlassen, andererseits. Diese Differenzierung schütze ,vor der Gefahr, die Organisation zu reifizieren, sie als eine überindividuelle Einheit mit einer Existenz und einem Verhalten unabhängig von dem Verhalten ihrer Mitglieder zu behandeln“ (Simon 1981: 273). ${ }^{32}$

Richard M. Cyert und James G. March (1963: 25-33) setzen der problematischen Annahme eines quasi kollektive Ziele setzenden „,metaphysischen Konzerngeistes“ (Scott/Davis 2007: 186) ihr Konzept der „Koalition“ entgegen. Dies macht sich zur Aufgabe, zu klären, wie und von wem Organisationsziele gesetzt werden. Dem Konzept zufolge stellen Organisationsziele das Ergebnis eines Verhandlungsprozesses unter den ähnliche Interessen verfolgenden Mitgliedern und Gruppen einer Organisation dar, die als eine „Koalition“ betrachtet wird. Das Konzept verdeutlicht, dass einzelne Personen und Gruppen Interessen haben und dass Organisationen Orte sind, an denen auf Basis von Aushandlungen akzeptable Vorgehensweisen vereinbart werden und auch neue Interessen entstehen. Dabei wird nicht davon ausgegangen, dass alle beteiligten Einzelpersonen und Gruppen gleich stark sind, auf Augenhöhe und harmonisch (inter-)agieren, wenn sie versuchen, ihre Interessenpräferenzen der Organisation aufzuerlegen bzw. eine gemeinsame Grundlage

\footnotetext{
${ }^{32} \mathrm{Ob}$ und inwieweit dies auch für die rational strukturierten, klar auf Effizienz ausgerichteten Organisationen gelten soll, bleibt offen und kann an dieser Stelle nicht weiter verfolgt werden.
} 
für gemeinschaftliches Handeln zu entwickeln. Damit wird ebenfalls unterstrichen, dass die individuellen Interessen der Mitglieder und Gruppen von den Zielen zu unterscheiden sind, die der Organisation Orientierungshilfen geben und zugleich Beschränkungen setzen.

Anhand der nachvollzogenen Überlegungen wird deutlich, dass die eingangs für die Konturierung der Organisationsziele in Anspruch genommenen drei Organisationsparadigmen nach Scott und Davis, so kategorisch wie ursprünglich gedacht, nicht mehr zu denken sind.

Darüber hinaus ist vor dem Hintergrund des Dargelegten zu bedenken, dass das individuelle Verhalten durch die Existenz unterschiedlicher sozialer Rollen beeinflusst wird, womit sich behaupten lässt, dass individuelle Motive von Organisationszielen zu unterscheiden sind. Dieser Gedanke wird implizit auch von Organisationstypologien mitgetragen, die herausstellen, dass lediglich in einer spezifischen Kategorie von Organisationen - darunter auch religiöse - individuelle Mitgliedermotive und Organisationsziele zusammenfallen. In der von Peter M. Clark und James Q. Wilson (1961: 137-152) vorgenommenen Unterscheidung von drei Organisationsarten betrifft dies den Typus der zielgerichteten oder ideologischen Organisationen, zu denen sie Wohlfahrtsverbände, gemeinnützige Vereine und soziale Einrichtungen zählen. ${ }^{33}$ Diese Organisationen zeichnen sich durch eine ausgeprägte weltanschauliche oder religiöse Wertebasis aus (vgl. auch Klöckner 2016: 189 ff.), die als solche als Anreiz für die Übernahme freiwilliger, unbezahlter Tätigkeiten in der Organisation fungiert; Anreize (im-)materieller Natur erscheinen in religiösen Organisationen hingegen eher nachrangig zu sein (4.4.2). Auch andere Organisationstypologien (Parsons 1960; Etzioni 1975; Blau/Scott 1962) konturieren für ihre Kategorisierungen divergente Ziele von Organisationen, die wiederum verschiedenartige Motivationen, intrinsische Interessen oder als wichtig erachtete Werte der Beteiligten für deren Mitwirkung voraussetzen. Auch die im Rahmen dieser Arbeit interessierenden religiösen Organisationen werden in solchen Typologien berücksichtigt und mit der Schaffung, Erhaltung und Tradierung eines kulturellen,

\footnotetext{
${ }^{33}$ Neben den zielgerichteten bzw. ideologischen Organisationen nennen Clark und Wilson utilitaristische bzw. nutzenorientierte Organisationen, zu denen nach ökonomischem Profit strebende Wirtschaftsunternehmen gehören. Darüber hinaus bilden die Autoren eine Kategorie für solidarische Zusammenschlüsse, zu denen sie Schulen, Universitäten, soziale Einrichtungen und auch Wohlfahrtsverbände zählen; diese verfolgten über die Gewinnerzielung hinausgehende anders gelagerte, wertebasierte Ziele, wie etwa die Vermittlung von Bildung oder politischem und sozialem Engagement. Diese Kategorie ist nicht trennscharf von den zielgerichteten oder ideologischen Organisationen abzugrenzen; bei letzteren steht jedoch die religiöse Wertebasis im Zentrum individuellen und kollektiven Handelns.
} 
normativen, wertebezogenen oder solidarisch ausgerichteten Systems für individuelle Bedürfnisbefriedigungen assoziiert (Parsons 1960: 44 ff.; Etzioni 1975: 40 ff.; Blau/Scott 1962: 40 ff.).

\section{Spezifika religiöser Organisationen}

In diesem Zusammenhang sind in Bezug auf die Zweckorientierung religiöser Organisationen aus theoretischer Perspektive die spezifischen Eigenarten religiöser Vereinigungen genauer zu betrachten. In Hinblick auf die Frage, was verhandelbar und daher von den Akteuren prozesshaft zu entwickeln und was nicht verhandelbar ist, sind die religiösen migrantischen Organisationen besonders interessant. Einerseits geht es um die einzelnen Mitglieder, mit ihren subjektiven Erfahrungen, Wünschen, Nöten und Bedürfnissen; andererseits ist gerade bei Zusammenschlüssen, die letztlich durch Religion bzw. durch religiös motivierte Weltbilder und Deutungsmuster gestiftet sind, am ehesten von überindividuellen Formaten des Denkens, des Handelns und des Empfindens auszugehen.

Niklas Luhmann zufolge dient der binäre Code der Religion - die Unterscheidung von Immanenz und Transzendenz (Luhmann 1977: 46 f., 2002: 77) - der „Bestimmung des Unbestimmbaren“(Luhmann 1977: 200) und der Bearbeitung und zugleich auch Produktion von Kontingenz (ebd.; Luhmann 2002b: 118 f.). Kontingenz bedeutet das Bewusstsein, dass alles immer auch anders sein und entschieden werden könnte (Krech 2018: 54, 56; s. auch Pollack 1995: 184) ${ }^{34}$. Religion übernimmt damit die Funktion der Sinnstiftung durch die Kommunikation über Sinn: „Religion garantiert die Bestimmbarkeit allen Sinnes gegen die miterlebte Verweisung ins Unbestimmbare“ (Luhmann 2002b: 127). Dadurch kommt eine gewisse Sicherheit ins Spiel, eine Glaubens-Gewissheit, die durch nichts anderes ersetzt werden kann, zum anderen aber auch eine gewisse ,Unschärfe', die im Grunde ein weiteres (viertes) Organisationsparadigma konstituiert, das durch die Präferenz des religiösen Habitus präfiguriert ist. Dies wirft einige Schwierigkeiten auf, gehört aber zum Kern des in dieser Arbeit anvisierten Forschungs(gegen)standes.

Angesichts der (zivil-)gesellschaftlichen - und selbsterhaltenden - Funktion von Religion wäre hinsichtlich der Performanz religiöser Organisationen theoretisch von mangelhaften zweckorientierten Kriterien auszugehen. Diesbezüglich sprechen Nicholas J. Demerath und Terry Schmitt (1998: 392) von den ,nicht greifbaren Zielen" und „nicht greifbaren Mitteln" religiöser Organisationen (vgl. auch Beckford 1975: 22 ff.; Clark/Wilson 1961: 147). Diese „Invisibilisierung“ bzw. Externalisierung von Kontingenz in religiösen Organisationen durch die Bereitstellung von

\footnotetext{
${ }^{34}$ Niklas Luhmann formuliert prägnant: ,,kontingent ( = unnotwendig, auch anders möglich)“ (Luhmann 1972: 246).
} 
Sinnformen, die sich auf das Transzendente beziehen, stünde der Abarbeitung von Unsicherheit und Kontingenz durch Entscheidungsverknüpfungen in ,säkularen * Organisationen entgegen. „Religiösen Organisationen steht es damit nicht einfach frei, ihre zentralen Strukturen qua Entscheidung zu ändern. Der Funktion des Religiösen könnte das im Kern widersprechen, nämlich da, wo Kontingenz gerade an der Stelle geltend gemacht würde, an der es sie zu neutralisieren gilt" (Petzke/Tyrell 2012: 290). Demerath und Schmitt (1998: 393) verweisen in diesem Zusammenhang auf historisch bedingte Kontinuitäten und Zwänge, die für eine religiöse Organisation etwa in Form von offenbarten heiligen Texten, tradierten Ereignissen oder Autoritäten überzeitlich relevant sein können und die - auch bei generell gegebener Zukunftsorientierung - die organisationale Performanz bestimmen. Durch die Beschäftigung mit Kontingenz - ohne diese auflösen zu können - schafft sich das religiöse System insofern seine eigene Basis und die Voraussetzung für seine fortdauernde Existenz (Pickel 2017: 388). Dieser Mechanismus dürfte in religiösen Organisationen in viele Richtungen Auswirkungen haben; er betrifft Kommunikationsformen, Entscheidungs- und Steuerungsprozesse, programmatische Inhalte und nicht zuletzt die Personalrekrutierung (Petzke/Tyrell 2012: 292). Je nach Religion bzw. Kirche verlaufen solche Vorgänge, die sich auch in Vereinsstrukturen spiegeln, freilich sehr different.

Die Annahme, dass dadurch die Zweckorientierung eines religiösen Zusammenschlusses (Vereins) mangelhaft ausfällt, greift in der praktischen Realität jedoch zu kurz. Denn die Ziele höherer, transzendenter Ordnung - die „Transzendierung, im Sinne der Verlagerung der Verantwortung ins Jenseits“ (Pickel 2011:21) - bedürfen konkret erfahrbarer Pendants in irdischen, immanenten, ,lebenswirklichen ' Verhältnissen. Eine gläubige Person benötigt Praktiken und eine Gemeinschaft, um das Religiöse im Diesseits erfahrbar zu machen. Für das Ausleben und die Bestätigung des individuellen Glaubens bedarf es durch eine religiöse Vereinigung vorgehaltener Gelegenheitsstrukturen sowie der dort gewährleisteten Kommunikabilität, wie etwa die sinnstiftende, durch Gebet und Ritual gegebene religiöse Praxis (Pollack 2018: 38 f.; Tyrell 2008: 264 f.; Krech 1999: 31 f.). Religiöse Überzeugungen und Praktiken finden ihren Ausdruck ebenfalls in zahlreichen (zivil-)gesellschaftlich wirksamen Aktivitäten: in karitativen Dienstleistungen, in diakonischen Angeboten, im Rahmen der Flüchtlingshilfe oder eines politischen Mitspracheanspruchs (3.1). In diesem Zusammenhang ist auf die von Detlef Pollack für religiöse Inhalte und Formen als charakteristisch pointierte ,,paradoxale Sinneinheit“ (Pollack 2018: 39) zu verweisen: „Charakteristisch scheint zu sein, dass Religionen das Transzendente nicht nur als das Andere des Immanenten, sondern auch als das Präsente, kommunikativ Erreich- und Erfahrbare, lebenspraktisch Relevante behandeln. Ein wichtiges Merkmal religiöser Inhalte und Formen scheint also in der Gleichzeitigkeit 
von Transzendenz- und Immanenzbezug, von Unbestimmtheit und Bestimmtheit, Unzugänglichkeit und Konkretion zu bestehen“ (ebd.: 43; Herv.: d. Verf.).

Die subjektive Glaubensdimension und die Einbindung des Individuums in eine religiös-moralische Wertegemeinschaft komplementieren und bedingen einander folglich gegenseitig. Sie sind insofern zusammenzudenken. Damit können religiöse Zwecke im profanen Alltag durchaus konkretisiert und greifbar gemacht und seitens religiöser Vereinigungen als solche formuliert werden (vgl. auch Beckford 1975: 23 f.). So ist z. B. bei religiösen Organisationen, die zuvorderst die Verkündigung und Verbreitung ihrer Glaubenslehre unter Anders- oder Nichtgläubigen und deren Bekehrung - also die Missionierung - verfolgen, von einer expliziten, die organisationale Performanz lenkenden Zielsetzung auszugehen. Ein Blick auf die Zwecke und Zielsetzungen islamischer Vereinigungen ergibt in konkreter Hinsicht folgendes exemplarisches Bild: In den unterschiedlich ausführlichen - oder als Selbstdarstellung deklarierten - Satzungen wird der Fokus auf religiöse Dienstleistungen wie religiösen Praktiken (tägliches fünfmaliges Gebet in der Moschee, Freitagsgebet), seelsorgerischer Betreuung, Koran- und Religionsunterricht, Pilgerfahrten etc. gelegt, aber auch andere Aufgaben wie Bildungs-, Jugend- und Antidiskriminierungsarbeit u. v. a. m. werden aufgenommen (Lemmen 2002: 28; s. z. B. IGMG 2015). Hier zeigt sich exemplarisch die Möglichkeit der Konkretisierung religiös verankerter bzw. religiös konnotierter Organisationsziele.

In diesem Zusammenhang sind auch die rechtlichen Bestimmungen zu Vereinszwecken im BGB relevant, die steuerrechtliche Privilegien ermöglichen und auf die im Folgenden näher einzugehen ist.

\section{Formalrechtliche Bestimmungen im Bürgerlichen Gesetzbuch (BGB)}

Vereine haben per definitionem einen gemeinschaftlichen ideellen Zweck zu verfolgen und diesen in einer Vereinssatzung festzulegen ( $\$ 57$ BGB). „Mit dem Vereinszweck soll angegeben werden, welche Ziele der Verein verfolgt und was durch den Verein erreicht werden soll. Er ist der Leitsatz für die Vereinstätigkeit“ (BMJV 2016: 16; Herv. weggel.). Häufig konzentriert sich der Hauptzweck von Vereinen auch lediglich auf die gemeinsame Ausübung von kulturellen oder Freizeitinteressen und damit auf Geselligkeit und Konvivialität (BMFSFJ 2017: 75).

Vereine sind auf das Grundgesetz verpflichtet und dürfen sich nur in diesem Rahmen bewegen. Ein „Verein zur Errichtung eines islamistischen Gottesstaats“ oder ein „Verein zur Erniedrigung des weiblichen Geschlechts“ wird aus verfassungsrechtlichen Gründen nicht zugelassen werden. Das Vereinsrecht thematisiert 
weitere Rahmenbedingungen und es enthält - neben Hinweisen zur demokratischen Form des Prozedere - Aussagen über die Rechte und Pflichten der Mitglieder.

Eine auf der Gründungsversammlung zu beschließende Vereinssatzung ist Voraussetzung für die Eintragung in das Vereinsregister und die Rechtsfähigkeit des Vereins ( $\$ 21 \mathrm{BGB})$. In freiwilligen Vereinigungen erfolgt die Zielbestimmung des Zusammenschlusses also im Konsens der Mitglieder, was in gewinnorientierten Unternehmen nicht der Fall ist. Der besondere Stellenwert der Mitglieder in Bezug auf die Bestimmung und Konzertierung des Vereinszwecks im Rahmen einer demokratischen Entscheidungsstruktur (Horch 1983) wird auch darin deutlich, dass jegliche Änderung des Vereinszweckes der Zustimmung aller Mitglieder auf der Mitgliederversammlung bedarf ( $\$ 33$ Abs. 2 Satz 2 BGB). Insofern kann angenommen werden, dass Ziele der Organisation und Mitgliederinteressen in freiwilligen Vereinigungen in idealtypischer Hinsicht deckungsgleich sind: Denn die Mitglieder haben ein Interesse an den Vereinszielen, die sie mitbestimmen dürfen und das in der Satzung oder anderen verschriftlichten Grundsatzprogrammen festgelegte Ziel der Vereinigung ist, dass eine gemeinsame Verfolgung und Vertretung der Interessen aller Mitglieder zum Nutzen aller verwirklicht werden soll (vgl. Blau/Scott 1962: 42-49; Weber 1985/[1922]: 21 f.).

Die Tätigkeiten des Vereinsvorstandes - des geschäftsführenden Organs - umfassen die Förderung des Vereinszwecks, sofern per Satzung oder Gesetz damit nicht ein anderes Organ betraut wurde ( $\$ 27$ Absatz 3 BGB). Die Vereinsaktivitäten müssen also den Vereinszweck unterstützen, der steuerlich begünstigt werden kann, wenn die Satzung und Tätigkeiten bestimmten Anforderungen entsprechen. Von Steuervergünstigen können insbesondere durch die Finanzverwaltung als ,gemeinnützig“ anerkannte Vereine profitieren. Die Bedingungen für die Erlangung des Gemeinnützigkeitsstatus sind in der Abgabenordung (AO) festgelegt (AO $\S 51$ ff.). Die Ziele des Vereins dürfen nicht wirtschaftlich, auf Gewinn ausgerichtet sein, sondern müssen sozialen, dem allgemeinen Wohl dienenden Aufgaben entsprechen, wodurch sich der ideelle oder Idealverein als Non-Profit-Organisation qualifiziert. Grundlegende Voraussetzung für die Erlangung des Gemeinnützigkeitsstatus ist die Verfolgung gemeinnütziger, mildtätiger oder kirchlicher Zwecke (§§ 52-54 $\mathrm{AO})$, die selbstlos ( $\$ 55 \mathrm{AO})$, ausschließlich ( $(56 \mathrm{AO})$ und unmittelbar $(\S 57$ AO) realisiert werden müssen. ${ }^{35}$ Die Tätigkeiten gemeinnütziger Vereine konzentrieren sich auf die selbstlose, d. h. karitativ-uneigennützige Unterstützung der allgemeinen Öffentlichkeit auf materiellem oder geistig-sittlichem Gebiet ( $\$ 52$ AO Gemeinnützige Zwecke) oder auf die Unterstützung von hilfsbedürftigen

\footnotetext{
35 Die zur Erlangung des Gemeinnützigkeitsstatus zu erfüllenden formalen Bedingungen werden häufig als zu strikt und einengend empfunden (Jachmann 2011: 114).
} 
oder einkommensschwachen Menschen ( $\$ 53$ AO Mildtätige Zwecke); des Weiteren betreffen sie Religionsgemeinschaften des öffentlichen Rechts im Rahmen kirchlicher Tätigkeit (§ 54 AO Kirchliche Zwecke) (s. hierzu auch Jachmann 2011).

In diesem Kontext ist anzumerken, dass Satzungen häufig recht ambitionierte Zieldeklarationen enthalten, die sich auch in Leitbildbroschüren oder Selbstdarstellungen der Organisationen spiegeln (Preisendörfer 2016: 68). Für alle Organisationstypen übernehmen die Ziele eine Legitimationsfunktion gegenüber den Mitgliedern und der Organisationsumwelt, die die organisationale Performanz (im Nachhinein) rechtfertigen, aber auch ein Scheinbild konstruieren können (4.5). Inwieweit in einer Vereinssatzung schriftlich fixierte Ziele in der Praxis tatsächlich verfolgt und umgesetzt werden, wird von Fall zu Fall und je nach Grad an Konkretion unterschiedlich geartet sein und amtlich nur hinsichtlich des Status der Gemeinnützigkeit kontrolliert. Ob sie in der Summe mehr den Implikationen von bonding oder mehr von bridging (4.2.3) entsprechen, wird im empirischen Teil dieser Arbeit separat und detailliert untersucht (III. 7.2).

Es lässt sich somit resümieren, dass Organisationsziele als Maßstab für die Auswahl von Handlungsoptionen dienen. Damit kommt ihnen eine kognitive (rationale) Funktion zu. In gewinnorientierten Unternehmen liefern Ziele Kriterien für die Bewertung von Leistungen, Strategien, Programmen und Mitarbeitern. Die Implementierung und Erreichung der gesetzten Ziele werden durch ökonomische Tauschbeziehungen gesteuert und streng kontrolliert. Ziele sind aber auch mit menschlichen, sozialen und emotionalen Aspekten verknüpft. In nicht profitorientierten Zusammenschlüssen können die Aufrechterhaltung der Organisation und die Durchsetzung ihrer Ziele durch geteilte, internalisierte Überzeugungen und Normen gesichert werden, die mit einem bewussten Streben nach der Erlangung eines für die eigene Lebensführung hoch erachteten (möglicherweise transzendenten oder symbolischen) Ziels verknüpft sind. Insofern ist eine MSO, die sich als religiösmoralische Wertegemeinschaft versteht, nur ein Spezialfall von Organisationen, die sich gerade in Bezug auf das Handeln selbst einem Wertesystem verpflichtet fühlen. Entscheidend ist, dass die Akteure für sich selbst individuell und zugleich für das Kollektiv, eine tätige Praxis ableiten. So gesehen verkörpert sich in den Zielen, die eine Organisation proklamiert, einerseits eine praxisbezogene Vorstellung, von dem, was man will, und in dem, was dann tatsächlich getan wird, ihre Performanz.

\subsubsection{Ressourcenausstattung}

Sind ohne Organisationsziele weder konkrete Leitlinien noch Maßstäbe und Motivationen möglich, so bestimmt die jeweilige Ressourcenausstattung die 
Handlungsfähigkeit einer Organisation. Ressourcen umfassen den Grundbestand der für eine Organisation wesentlichen Komponenten, auf den sie zurückgreifen bzw. den sie mobilisieren kann und der für ihre Existenz und ihr Wirken wesentlich ist. Ressourcen können strukturiert werden nach inneren und äußeren, nach finanziellen und materiellen, nach personellen und ideellen, schließlich nach individuellen und kollektiven.

Für Pugh et al. (1963: 311 ff.) stellen finanzielle und materielle Mittel neben personellen und ideellen Ressourcen grundlegende Aktiva dar, die bei der Analyse von Organisationen bedeutsam sind. ${ }^{36}$ Pugh et al. fassen diese vier Aspekte in zwei Hauptkategorien zusammen: 1.) Personal- und Ideenressourcen und 2.) Material- und Kapitalressourcen. Die personellen Ressourcen umfassen die Mitglieder als freiwillig Engagierte, bezahltes Personal oder anderweitig Beteiligte. Diese Personen sind in unterschiedlichen Intensitätsgraden, unter ideellen Gesichtspunkten und Bedarfen für die Umsetzung der Organisationsziele verantwortlich.

$\mathrm{Zu}$ den materiellen Ressourcen gehören Gebäude oder Räumlichkeiten, Grundstücke, Anlagen, Lagerbestände, Einrichtungsgegenstände sowie die Ausstattung mit Technik und gegenständlichen Materialien. Mögliche Kapitalressourcen umfassen verfügbares Bargeld, Wertpapiere, Kredite, Investitionen, Spenden, Einnahmen durch Veranstaltungen mit Eintrittsgeldern, den Verkauf von Waren, Verdienste durch gastronomischen Betrieb oder das Angebot von Pilgerfahrten sowie Mittel aus öffentlicher Förderung.

Dass Organisationen für Bestand und weiteren Ausbau auf Ressourcenzuflüsse aus der Umwelt angewiesen sind, ist eine wesentliche Grundannahme der Theorie der Ressourcenabhängigkeit (,resource dependence theory“, kurz: RDT), die in den 1980er Jahren erhöhte Aufmerksamkeit erfuhr (siehe v. a. Pfeffer/Salancik 1978; Pfeffer 1987). Je nachdem, wie stark diese Abhängigkeit ausfällt, stellt sie einen zentralen Einflussfaktor dar. Die jeweiligen Ressourcenzugänge schwanken allerdings und sind oft unberechenbar, sodass es ein Hauptanliegen von Organisationen ist, dass diese Unsicherheit absorbiert und der Ressourcenzufluss dauerhaft sichergestellt werden kann (Preisendörfer 2016: 151). Denn Ressourcenstrukturen bilden den Handlungskontext für Organisationen: Einerseits eröffnen sie Gelegenheiten und Opportunitäten, andererseits können sie Restriktionen (,constraints“) darstellen und beeinflussen bzw. begründen damit das organisationale Handeln.

\footnotetext{
${ }^{36}$ Die Autoren um Derek S. Pugh gehören zur sogenannten „Aston-Gruppe“ und sind damit der Kontingenz-Theorie oder dem ,situativen Ansatz“ zuzuordnen (4.3, Tab. 4.2). Der Kontingenztheorie zufolge beeinflussen und prägen spezifische interne Situationsparameter sowie externe Kontextfaktoren die Ausgestaltung der einzelnen Dimensionen der formalen Organisationsstruktur als die abhängige Variable (Preisendörfer 2016: 83 ff.).
} 
Aus spezifischen Ressourcenbeschränkungen resultieren Bedarfe, die die Organisation selbst als solche registrieren, artikulieren und durch das Ergreifen von Maßnahmen aktiv bearbeiten kann (III. 6.1).

Die Studie Islamisches Gemeindeleben in Deutschland (Halm et al. 2012a) stellt heraus, dass die Größe und die Ressourcenausstattung (u. a. in Form vorhandener Räumlichkeiten, Abteilungen oder Immobilienbesitz) zentrale Einflussfaktoren für den Angebotsumfang der Organisationen darstellen (ebd.: 7). Die Ergebnisse stimmen mit organisationssoziologischen Annahmen überein (Pugh et al. 1969). Der Studie zufolge ist „[d]as Vorhandensein personeller, infrastruktureller und finanzieller Ressourcen" die wichtigste Voraussetzung dafür, „dass Gemeinden religiöse wie auch integrationsrelevante Angebote unterbreiten, Kooperationen mit der Aufnahmegesellschaft eingehen und Strukturen herausbilden, die es ihnen ermöglichen, auf durch intergenerationalen und sozialen Wandel veränderte Bedarfe ihrer Klientel zu reagieren. Die Ressourcen sind dabei oft eine notwendige (nicht allein hinreichende) Bedingung für die Erfüllung dieser Aufgaben“ (ebd.: 117; Herv.: d. Verf.). Folglich ist zu erwarten, dass i. d. R. proportionale Zusammenhänge vorzufinden sind: Je ressourcenstärker eine Organisation ist, desto mehr Leistungen kann sie erbringen.

Grundsätzlich stellt sich die Ressourcenfrage in freiwilligen Vereinigungen anders als in solchen, die auf Wirtschaftlichkeit und Gewinn ausgerichtet sind. Im Vordergrund steht nicht die Implementierung einer ökonomischen Effizienzund Profitperspektive mit entsprechend zu erreichenden Zielen und Erfolgen. Es handelt sich vielmehr um genuine, tendenziell demokratisch strukturierte Mitgliederorganisationen ohne dezidiert ökonomische Anreize für Mitgliedschaft und Mitarbeit, sodass die ideelle Motivation zur entscheidenden Ressource wird. Zentral ist der Aspekt der Freiwilligkeit: Die freiwillig-unbezahlte, ehrenamtliche „Teilnahme an der Arbeit und am Entscheidungsprozeß ist für jede Vereinigung eine wesentliche Ressource“ (Horch 1985: 261). Das ist v. a. auch rechtlich von Belang. Denn formalrechtlich wird eine Vereinigung erst dann handlungsfähig, wenn der Vereinsvorstand bzw. entsprechende Organe gewählt oder ernannt wurden ( $\$ 26$ Abs. 1 BGB) und die Mitgliederversammlung mit ihrem Mandat für Beschlussfassungen ( $\$ 32$ Abs. 1 BGB) auch tatsächlich aktiv wird. Damit kommt den Mitgliedern und Ehrenamtlern als Hauptressource für den Bestand und die Funktionsfähigkeit von Vereinen und (Interessen-)Verbänden eine konstitutive Bedeutung zu (Streeck 2017/[1987]: 528 ff.; Horch 1983: 16, 165). Dabei ist selbstverständlich von unterschiedlich stark ausgeprägter Mitwirkung in Form freiwilliger Engagements auszugehen (Heinze 2011; Zimmer 2011).

Angesichts der genannten Größen, die die Ressourcenausstattungen und mithin Performanzen der migrantischen Vereinigungen mitbestimmen, soll folgende 
Hypothese aufgestellt und ihre Gültigkeit mit den im Rahmen dieser Arbeit erhobenen Daten - an späterer Stelle - geprüft werden:

H2: Insbesondere die mit genügend Geld und personellen Ressourcen (Mitglieder, Hauptamtliche und Engagierte) ausgestatteten MSO sind imstande, ein breites Spektrum an Aktivitäten und Dienstleistungen anzubieten und Kooperationen einzugehen.

Philippe C. Schmitter und Wolfgang Streeck (1999/[1981]: 19) stellen für nicht profitorientierte Interessenverbände die Notwendigkeit heraus, dass sie ihren Mitgliedern adäquate Anreize zu bieten haben, um über den Bezug von Ressourcen das Überleben und Wachstum der Organisation zu sichern. Zudem muss über die Mitglieder der Zugang zu öffentlichen Einrichtungen gewährleistet werden, um dadurch zweckentsprechende, existenzsichernde Ressourcen wie Anerkennung, Status, finanzielle Subventionen und weitere Zugeständnisse zu erlangen. Schmitter und Streeck machen damit auf zwei wesentliche „,Logiken“ des Austausches“ - der „Logik der Mitgliedschaft“ und der „Logik des Einflusses“ - als ein wesentliches Charakteristikum von Interessenverbänden aufmerksam ${ }^{37}$. Das ist $u$. a. deshalb von großem Interesse, weil es zwischen den islamischen Verbänden aufgrund einer Konkurrenzsituation einen starken Wettbewerb um Mitglieder gibt (Rosenow-Williams 2012: 48, 67). Dabei betrifft die Konkurrenz keineswegs nur die Frage der personellen Ressourcen, sondern ganz wesentlich diejenige von öffentlich akzeptierter Repräsentation und Legitimität (Halm et al. 2012a: 26, 32). Welche Organisation wen und was am besten repräsentiert - und mit welcher Legitimität -, ist durchaus umstritten. Ein wettbewerbsorientiertes Verhältnis ist jedoch nicht nur unter islamischen Verbänden, sondern auch zwischen religiösen und nicht-religiösen MSO und zunehmend auch zwischen islamischen und den deutschen Wohlfahrtsorganisationen auszumachen (Rosenow-Williams 2012:

\footnotetext{
${ }^{37}$ Unternehmens- und Interessenverbände ,müssen sich einerseits selbst strukturieren und so handeln, dass sie ihren Mitgliedern genügend Anreize bieten, um von ihnen zur Sicherung des Überlebens oder auch Wachstums ausreichende Ressourcen zu erhalten. Andererseits müssen sie so organisiert sein, dass sie [...] [ihre Mitglieder] in die Lage versetzen können, Zugang zu öffentlichen Einrichtungen [...] zu erhalten und auf diese Einfluss auszuüben, um aus diesem Austausch Ressourcen herauszuziehen (Anerkennung, Toleranz, Zugeständnisse, Subventionen usw.), die es ihnen ermöglichen, zu überleben und zu prosperieren. Diese beiden ,Logiken“ des Austausches bezeichnen wir als , die Logik der Mitgliedschaft ‘ und die ,Logik des Einflusses', und unsere ursprüngliche Hypothese ist, dass die organisationalen Eigenschaften [jedes Unternehmens- oder Interessenverbandes] analytisch als das Ergebnis - in der Regel ein kompromittiertes - dieser Interaktion betrachtet werden können“ (Schmitter/Streeck 1999/[1981]: 19; Übers.: d. Verf.).
} 
437 ff., 469). Die nicht gewinnorientierten Organisationen haben ihren Mitgliedern also Anreize für die Beteiligung zu bieten (4.4) und ihre Interessen mit denen der Umwelt auszubalancieren (4.5).

Ressourcen zu beschaffen bzw. zu mobilisieren stellt sich - aufs Ganze gesehen - für die sehr heterogen aufgestellten Freiwilligenorganisationen und insbesondere für die MSO in Deutschland sehr unterschiedlich dar. Die Landschaft der MSO ist sehr vielfältig, sie verfügen über sehr unterschiedliche Ressourcenausstattungen, nicht alle profitieren von Fördermaßnahmen, geschweige denn von politischer Aufmerksamkeit. Dies ist nicht zuletzt darauf zurückzuführen, dass die Förderschwerpunkte, -maßnahmen, -richtlinien und jeweiligen situativen und finanziellen Gegebenheiten auf Bundes-, Landes- und kommunaler Ebene sehr disparat ausfallen. Ein gleichberechtigter Zugang zu Fördermitteln ist nicht gewährleistet, da häufig ressourcenstarke, professionalisierte MSO und bundesweit tätige Migranten(dach)verbände von den Fördermaßnahmen profitieren bzw. diese laut den formellen Förderkriterien explizit die Zielgruppe spezifischer Förderprogramme darstellen (BT 2016b: 2; BT 2018: 2). Kleineren, ressourcenärmeren MSO mangelt es hingegen ,an Wissen, Zeit und Kompetenzen für die Antragstellung um Fördermittel, [...] an Zugängen zu den entscheidenden Netzwerken und Geldgebern, und [...] an Möglichkeiten von Ko-Finanzierungen“ (Weiss 2013: 26; s. auch Hunger/Metzger 2011: 74 f.).

Kritische Äußerungen betroffener Organisationen bleiben deshalb nicht aus. In dem für den empirischen Teil dieser Arbeit verwendeten Fragebogen wurde der zur Verfügung gestellte Platz für Anmerkungen und Kommentare von den befragten MSO genutzt, um auf die schwierigen Akquisebedingungen von Fördermitteln Bezug zu nehmen. So wurde beispielsweise angemerkt: „Wir wissen teilweise, wo wir Fördergelder beantragen können, doch der zeitliche Aufwand ist so hoch, dass wir es mit unseren ehrenamtlichen Mitarbeitern nicht stemmen können“ (ID 50). Und eine verbandsunabhängige islamische Gemeinde konstatierte: „Der Zugang zu Fördermitteln für islamische Gemeinden, die unabhängig sind, sollte einfacher sein und auch mehr geschätzt werden“ (ID 178).

Des Weiteren ist zu bedenken, dass MSO häufig über keine eigenen oder adäquaten Räumlichkeiten verfügen, was für vereinsinterne Versammlungen, die Umsetzung von Aktivitäten und geplanten Programmen ein großes Hindernis darstellt (vgl. z. B. Hunger/Metzger 2011: 61; Friedrichs et al. 2020: 79 f., 89 f.). Auch auf diese Problematik wurde im Rahmen der Erhebung seitens der befragten MSO in dem für Anmerkungen und Kommentare vorgesehenen Platz Bezug 
genommen: „Wir haben Probleme beim Erwerb bzw. Anmieten von Räumlichkeiten, die für unsere Aktivitäten besser geeignet sind! Wir haben keine ausreichenden finanziellen Mittel. Die Stadt verteilt keine Nutzungsgenehmigungen (bisher)“ $(\text { ID 191) })^{38}$.

Somit bleibt festzuhalten, dass für MSO in Deutschland unterschiedliche Möglichkeiten und Chancen für den Erwerb von Ressourcen bestehen, bei denen es neben finanzieller Unterstützung ganz erheblich immer zugleich um die Erlangung von offiziellem Status, Legitimität und Rechtssicherheit geht.

Mark C. Suchman (1995: 574) zufolge profitieren Organisationen insbesondere von Legitimität im Sinne gesellschaftlich allgemein als wünschenswert und angemessen wahrgenommener Handlungen, wenn sie sich in der Öffentlichkeit natürlich und bedeutungsvoll und in Bezug auf das Aufnahmeland mit kultureller Affirmation präsentieren. Der Zugang zu Ressourcen, über die noch nicht verfügt wird, stelle dann weitgehend ein Nebenprodukt dar (ebd.: 575 f.; 4.5). Naheliegend ist dann auch, dass das einer Organisation in der Öffentlichkeit durch andere Einrichtungen und Akteure entgegengebrachte Vertrauen eine bedeutsame Ressource darstellt (Rosenow-Williams 2012: 60). Wieder kann auf Anmerkungen der im Rahmen dieser Arbeit befragten MSO Bezug genommen werden. So wurde beispielsweise vermerkt, dass, ,[d]as größte Hemmnis der Vereinsarbeit [...] das mangelnde Vertrauen der Behörden gegenüber den Migrantenorganisationen" sei (ID 9). Und eine andere Person schrieb: „Ich hoffe auf MEHR VERTRAUEN seitens der Politik und Verwaltung zu uns Kulturschaffenden“ (ID 73; Herv. i. Orig.).

\subsubsection{Mitglieder}

Niklas Luhmann (1964, 2000, 2005a-c/[1975a-c]) hat sich intensiv mit der Bedeutung eines Faktors auseinandergesetzt, der bisher zu wenig zu Wort kam,

${ }^{38}$ Weitere die Existenz einer Raum-Problematik betreffende Kommentare lauteten:

„Unser andauerndes Problem ist, geeignete Räumlichkeiten für unsere Aktivitäten zu finden. Geeignete Räume sind teuer und schwer zu beschaffen. Es gibt in der Stadt kaum Möglichkeiten, ohne Kosten Räume für Kurse oder Aktivitäten für Kinder zu verwenden“ (ID 6).

„Wir brauchen Räumlichkeiten für unser Sommerferienprogramm. Viele afrikanische Familien können sich keinen Urlaub für ihre Kinder leisten. Darum organisieren wir jährlich ein Ferienspaßprogramm für die Kinder“ (ID 18).

„Unserer Organisation stehen keine Räumlichkeiten mehr zur Verfügung, sodass sie organisch nicht mehr aktiv ist“ (ID 110). 
nämlich mit der Mitgliedschaft. Seine Überlegungen haben organisationssoziologischen Ansätzen wichtige Impulse gegeben (Tacke 2010: 342). Daher sollen sie bei der Explikation des Basiselements „Mitglieder“ im Folgenden berücksichtigt werden. Allerdings beziehen sich Luhmanns Überlegungen überwiegend auf Arbeitsbeziehungen und sind daher für die in dieser Arbeit im Interessenfokus stehenden freiwilligen und religiösen Vereinigungen mit Einschränkungen und als Kontrastfolie verwertbar. Luhmanns Ausführungen werden daher von theoretischen Betrachtungen anderer Autoren (Barnard 1970; Horch 1983; Petzke/Tyrell 2012) sowie von vereinsrechtlichen Bestimmungen des Bürgerlichen Gesetzbuches (BGB; BMJV 2016) flankiert.

\section{„Selbstverpflichtung“ und ,Treuepflicht"“}

Mitglied in einer Organisation zu werden, z. B. in einem Verein, ist nach Luhmann eine freiwillige „entscheidungsfähige Angelegenheit“ (Luhmann 1964: 35), d. h. eine Begebenheit, für deren Motivation und Durchführung allein die Entscheidung der betreffenden Person maßgeblich ist, die Mitglied werden will. Die Wahl, Mitglied zu werden oder eben nicht, kann jedem, der dies tun will, als bewusste Entscheidung abverlangt werden, irgendeine Art von Zwang steht hier ganz außer Frage. Die Organisationen müssen ihrerseits mit ,entscheidbaren Entscheidungsprämissen“(Luhmann 2000: 239; Herv. i. Orig.) arbeiten. Sie formulieren bestimmte Voraussetzungen, Konditionen und Verhaltenserwartungen für Mitgliedschaften, an die sich die Mitglieder zu halten haben. Damit beanspruchen Organisationen, Maßstäbe für erlaubtes und unerlaubtes Handeln aufzustellen (Luhmann 1964: 29). Mit der Prüfung, ob die Gründe für eine Mitgliedschaft hinreichend plausibel sind, sie mit der Satzung, den erklärten Zielen etc. übereinstimmen, können Organisationen grundsätzlich über Ein- und Austritte von Personen bestimmen. Die Organisation schafft so Grenzen gegenüber der Außenwelt und Nicht-Mitgliedern (Luhmann 2000: 112, 2005a/[1975a]: 13 f.).

Luhmanns Überlegungen betreffen in erster Linie die Verbindlichkeit der Mitgliedschaft qua Mitgliedsrolle ${ }^{39}$ und festgelegter Mitgliedschaftsregeln. Verglichen mit der Nicht-Mitgliedschaft sei das Mitglied-Sein eine Sonderrolle, mit der man bestimmten Verhaltenserwartungen zu entsprechen hat. Das Organisationsmitglied sollte sich bewusst sein, dass es bei Nichtbeachtung von Vorgaben - wie etwa der Entrichtung von Beiträgen, dem Zweck der Organisation, damit

\footnotetext{
${ }^{39}$ Unter dem Begriff der „Rolle“ wird in der Soziologie ein „Bündel von Erwartungen [verstanden], die sich in einer gegebenen Gesellschaft an das Verhalten der Träger von Positionen knüpfen“, womit ,jede einzelne Rolle ein Komplex oder eine Gruppe von Verhaltenserwartungen“ (Dahrendorf 1965: 26) darstellt (s. auch Luhmann 2000: 81 f.).
} 
verbundenen Programmen und erwarteten Kommunikationsformen - die Organisation zu verlassen hat, da ,die Nichtanerkennung oder Nichterfüllung dieser Erwartung[en] mit der Fortsetzung der Mitgliedschaft unvereinbar ist" (Luhmann 1964: 38; Herv. weggel.). Die Mitgliedschaftsentscheidung setzt daher eine gewisse subjektive „Selbstverpflichtung“ (ebd.: 37; Herv. i. Orig.) voraus, die Mitgliedsrolle und die damit verknüpften Regeln anzuerkennen. Die Mitgliedschaft ist damit ein Akt von Commitment - das Sichbekennen und Sichverpflichten des Subjekts sind maßgeblich und ausschlaggebend für Eintritt und Verbleib in der Organisation: „Wer in ein formalisiertes System eintritt, [...] muß eine sichtbare Schwelle überschreiten und kann dies nicht tun, ohne etwas über sich selbst auszusagen. Er gibt also eine bestimmte Selbstdarstellung, teilt anderen sein Interesse am System und seine Bereitschaft mit, die Systemerwartungen anzuerkennen“ (ebd.). Dies realisiert sich innerhalb einer Vereinigung über die „Treuepflicht“, die von den Mitgliedern verlangt, nicht nur rechtzeitig den Mitgliedsbeitrag zu zahlen, sondern die „Interessen des Vereins zu fördern und vereinsschädigendes Verhalten zu unterlassen. Von den Mitgliedern wird auch die Bereitschaft zur Übernahme von Vereinsämtern erwartet“ (BMJV 2016: 46). Derlei Pflichten stehen natürlich auch Rechte der Vereinsmitglieder gegenüber, die im BGB niedergeschrieben sind. Eine zentrale Funktion der Gründungsmitglieder eines Vereins ist in deren Recht bzw. Pflicht zu sehen, im Rahmen eines offiziellen Gründungsaktes einvernehmlich über eine von sieben Mitgliedern zu unterschreibende Vereinssatzung zu entscheiden. Mit dieser gibt sich der Verein quasi eine rechtliche Ordnung, in der u. a. ein gemeinschaftlicher Vereinszweck festgelegt werden muss ( $\$ 57$ BGB). Die Satzung umfasst Bestimmungen zu Einund Austritten der Mitglieder, zu Mitgliedsbeiträgen, zur Bildung und Zusammensetzung des Vorstandes, zur Einberufung von Mitgliederversammlungen und das Beurkunden ihrer Beschlüsse ( $\$ 58 \mathrm{BGB}$ ). Mitglieder dürfen die Vereinseinrichtungen nutzen, an Vereinsveranstaltungen und Mitgliederversammlungen teilnehmen, sich zur Wahl eines Vereinsorgans aufstellen oder die Veranstaltung einer Mitgliederversammlung verlangen oder erzwingen ( $\$ 37$ BGB).

Die Mitgliedschaft in einem Verein kann durch die Beteiligung am Gründungsakt oder durch einen Aufnahmeantrag und eine Beitrittserklärung der sich bewerbenden Person erworben werden (BMJV 2016: 49). Erlöschen können die Mitgliedschaft und alle damit verbundenen Mitgliedsrechte und -pflichten jederzeit durch Austritt aus dem Verein ( $\$ 39$ Abs. 1 BGB), durch den Todesfall oder einen (automatischen) Ausschluss des Mitglieds aus dem Verein gemäß entsprechender Festlegungen in der Satzung, z. B. wenn das Mitglied seine Pflichten in schwerwiegendem Maße verletzt hat (BMJV 2016: 49). 
Luhmann konstatiert, dass eine Person, die trotz einer Nichtanerkennung von Mitgliedschaftsregeln dennoch Mitglied bleiben möchte, durch ihr nichtkonformes, regelwidriges Verhalten Ansehen, Glaubwürdigkeit und Respekt verliert und damit einen „Teil [ihres] Ichs, zu dem [sie] sich bekannt hatte“ (Luhmann 1964: 37). Die Möglichkeit, über eine Mitgliedschaft zu entscheiden, ist für die Organisation insofern konstitutiv, als sie gegenüber der Person, die Mitglied ist, eine Sinn- und Erwartungsgrenze zieht und ihr ein Feld des Erlebens sowie ein Feld für aktive Mitwirkung bietet; gegenüber Nicht-Mitgliedern zieht die Organisation hingegen eine Grenze (ebd.: 35).

\section{Rollensegregation in Arbeitsorganisationen (Luhmann)}

In Bezug auf formale Arbeitsorganisationen geht Luhmann von einer ,Trennung von Teilnahmemotivation und Leistungsmotivation" aus (Luhmann 1964: 104; Herv. i. Orig.; s. auch Luhmann 2005a/[1975a]: 13 f.). Persönliche Motivstrukturen und Beziehungen werden über die Mitgliedschaftsrolle von den Verhaltensanforderungen bzw. Zwecken der Organisation und dem Arbeitsverhältnis getrennt. Die Tochter des Geschäftsführers ist dementsprechend idealerweise eine Auszubildende unter vielen und erfährt die Organisation in ihrem Alltag als eine von anderen Systemen und Rollen abzugrenzende Einrichtung (vgl. Horch 1983: 98). Durch diese Rollensegregation können organisationsinterne und -externe Rollen, als potenziell unverträgliche Anerkennungsverhältnisse, auseinandergehalten und persönliche Ansprüche, die mit der Mitgliedschaftsrolle unvereinbar sind, abgewiesen werden (Luhmann 1964: 65). Verhaltensanforderungen, die von der Organisation ausgehen, und die je individuellen (privaten) Motive der Mitglieder können so unabhängig voneinander betrachtet und verändert werden, was nicht ausschließt, dass sie sich dauerhaft miteinander verknüpfen (Luhmann 2005a/[1975a]: 13; Luhmann 1964: 101).

Durch die Trennung von individuellen Motiven und organisationaler Zweckstruktur in einer Arbeitsorganisation sichert sich diese, z. B. hinsichtlich der Modifikation ihrer Ziele, eine gewisse Flexibilität im Inneren und ihre Anpassungsfähigkeit gegenüber dynamischen Entwicklungen nach außen. Gerade diese Flexibilität kann die Effizienz eines Unternehmens erheblich steigern (Luhmann 1964: 94 f., 100 ff.). Über die Zuordnung bestimmter Erwartungen, Aufgaben, Funktionen und Entscheidungsbefugnisse qua Mitgliedschaftsregeln wird das Verhalten der Mitglieder im Sinne des Organisationszweckes gelenkt und es ist möglich, langfristig ,hochgradig künstliche Verhaltensweisen“ (Luhmann 2005a/[1975a]: 14) herzustellen. Solange sich die Verhaltensanforderungen und die Attraktivität des Systems im Gleichgewicht halten und die Mitgliedschaft 
gegenüber einer Nicht-Mitgliedschaft, z. B. aufgrund von Gehaltszahlungen, als vorteilhafter wahrgenommen wird, ist eine Steigerung und Generalisierung der (Arbeits-)Motivation möglich: „Die Soldaten marschieren, die Schreiber protokollieren, die Minister regieren - ob es ihnen in der Situation nun gefällt oder nicht" (ebd.: 14).

Freilich muss man Luhmanns Überlegungen hinsichtlich ihrer Übertragbarkeit auf migrantische Vereinigungen mit Vorbehalt betrachten, denn er spricht hier von Arbeitsbeziehungen im Arbeitsverhältnis, nicht von zivilgesellschaftlichen Organisationen.

\section{„Doppelpersönlichkeit““ des Akteurs in der Organisation (Barnard)}

In Chester Barnards Beitrag zur Organisationssoziologie wird das von Luhmann thematisierte Problem der Rollendifferenz verschärft. Barnard, auf den Niklas Luhmann in seinen Ausführungen zur Trennung von Teilnahme- und Leistungsmotivation Bezug nimmt (u. a. Luhmann 1964: 96, 98, 100, 104; Luhmann 2000: 114), spricht von einer „Doppelpersönlichkeit“ des Akteurs in der Organisation, die sich in eine ,individuelle“ und eine „Organisationspersönlichkeit“ aufteilt (Barnard 1970: 82). Es gebe eine individuelle Person mit persönlichen Vorstellungen, Wünschen und Bedürfnissen und eine organisational eingebundene, die sich der Verwirklichung der Organisationsziele zu verschreiben versucht. Vor diesem Hintergrund spricht Barnard in Hinblick auf die Möglichkeit von sich in gewissen Grenzen vollziehenden Veränderungen in der Organisation, die von den Mitgliedern aufgrund von Uninteressiertheit mitgetragen werden können, von einer „Zone der Indifferenz“" (Barnard 1970: 143-145).

Barnard zufolge sind Zwecke kooperativer Anstrengungen sozial und unpersönlich und können sich von individuellen Zielen erheblich unterscheiden. Die Zielsetzung einer Organisation bestimmt den Rahmen für die Beitragsleistung und die Entwicklung der Organisationspersönlichkeit eines Individuums innerhalb einer Organisation, wobei das persönliche Verhalten (z. B. in einer militärischen Organisation) idealerweise von der sich mit dem Kooperationssystem identifizierenden Organisationspersönlichkeit dominiert wird. Die Vermittlung zwischen den Organisationszielen und individuellen Motiven erfolgt über die organisationsinterne Kommunikation, der damit eine zentrale Bedeutung zukommt (ebd.: 84). Denn die Verfolgung von Organisationszielen seitens der Mitglieder sollte als auch dem eigenen Vorteil dienlich erkennbar sein.

An diesem Punkt ist erneut das Verhältnis zwischen Organisationszielen einerseits und den beteiligten Individuen und deren Bedürfnisse andererseits angesprochen, das zu einer Klärung bzw. Bestimmung auffordert. Die Relation von 
Organisationszielen und individuellen Motiven der Mitglieder wurde auch bereits in dem Kapitel zu den Organisationszielen (4.3.1) thematisiert. Obgleich die Ziele von den Motiven zu unterscheiden sind, können sie insbesondere in freiwilligen und religiösen Vereinigungen sehr eng miteinander verknüpft sein, was im Folgenden noch einmal tiefergehend verdeutlicht werden soll.

\section{Mögliche Verquickung von Organisationszielen und Mitgliedermotiven}

Wenngleich Barnard für eine klare Trennung zwischen dem organisationalen Zweck und individuellen Motiven, zwischen subjektiver und Organisationspersönlichkeit plädiert, führt er eine wichtige Ausnahme von dieser Regel an, nämlich wenn die Realisierung eines Organisationsziels ,,selber zur Quelle persönlicher Befriedigung“ und ,zum einzigen oder auch nur zum wichtigsten individuellen Ziel“ (ebd.: 83; Herv. i. Orig.) wird. Dieses kann v. a. in religiösen, patriotischen oder politischen Zusammenschlüssen der Fall sein (Simon 1981: 61, 143 f.), in denen Ziele als direkte Anreizmittel fungieren und motivierende Funktionen übernehmen, da sich die Mitglieder mit den Zielen identifizieren und dadurch auch persönliche Bedürfnisse befriedigen können (vgl. auch 4.3.1). Insofern ist hier mit Martin Petzke und Hartmann Tyrell (2012) auf die sehr enge Verflechtung zwischen der Motivation der Mitglieder zur Beteiligung in einer religiösen Organisation einerseits sowie deren formaler Struktur und Zwecke andererseits zu verweisen: „Weder äußerer Zwang noch Geld, sondern nur die innere Affirmation des Evangeliums kann den genuinen Glauben bewirken und die Mitgliedschaft in der Glaubensgemeinschaft legitimieren" (Petzke/Tyrell 2012: 290 f.). Diese Aussage kann durchaus auch für andere Religionen, aber auch für politische oder andere profane wertebasierte Vereine Geltung beanspruchen. Bei diesen ist davon auszugehen, dass sich ihre Ausrichtungen, Zweckbestimmungen und Aktivitäten nicht ohne Weiteres und nicht ohne die Zustimmung der Mitglieder verändern lassen, da für die Mitglieder zentrale Anreize nicht mehr bestehen würden (ebd.: 291).

Die Feststellungen von Barnard, Petzke und Tyrell können auch auf Freiwilligenorganisationen im Allgemeinen übertragen werden. Auch in diesen sind individuelle Motive der Mitglieder und die Ziele der Vereinigung nicht ohne Weiteres voneinander zu trennen, denn die Identifikation mit den Zielen, Handlungsbereitschaft und Wertschätzung der durchgeführten Aktionen spielen als Gesamt von Anreizen eine kaum zu überschätzende Rolle ${ }^{40}$ (4.4). Im Gegensatz

${ }^{40}$ Auch Luhmann merkt an, dass die Zwecke insbesondere in Freiwilligenorganisationen eine motivierende Funktion haben; er verweist aber auf die mit einer Festlegung von Zwecken als Motivationsfaktoren für Mitglieder einhergehende Immobilisierung aufgrund der Problematik 
zu zweckrationalen und ökonomischen Kriterien folgender Mitarbeit in gewinnorientierten Unternehmen und der sich dort vollziehenden „Generalisierung oder ,Kapitalisierung ' der Motivation“ (Luhmann 1964: 95) beruht die Mitwirkung in freiwilligen Vereinigungen nicht auf monetären Anreizen zwecks Sicherung des Lebensunterhalts, sondern auf ehrenamtlichem, unbezahltem Einsatz (Horch 1985: 259 ff.).

Wenngleich eine Differenzierung zwischen Organisationszielen und Mitgliedermotiven theoretisch notwendig ist (4.3.1), sind Mitgliederinteressen und Organisationsziele in freiwilligen Vereinigungen in idealtypischer Hinsicht als deckungsgleich zu betrachten insofern, als individuelle innere Überzeugungen mit den kollektiven Anliegen einer Organisation korrespondieren, subjektive Motive und Ideale in den kollektiven Zielen wiedererkannt und sich diese zu einer eigenen Sache gemacht werden können (4.3.1); Ziele fungieren als direkte Anreizmittel und übernehmen motivierende Funktionen. Allerdings ist diese Identität von Mitgliederinteressen und Organisationszielen keinesfalls automatisch gegeben und es ist nicht davon auszugehen, dass der Vereinszweck allein ausreicht, damit sich Personen für eine Mitgliedschaft und Beteiligung in einem Verein entscheiden (Horch 1985: 259; s. hierzu die folgenden Abschnitt 4.3.3.1; 4.3.3.2; 4.4).

Für nicht gewinnorientierte Organisationen ist an dieser Stelle auf den zentralen Aspekt ihrer Abhängigkeit von den sich freiwillig und unbezahlt in die Organisation einbringenden Mitgliedern als eine Hauptressource herauszustellen, da diese durch ihre Aktivitäten, finanziellen Beitragszahlungen und als Legitimitätsquelle den Status und die Existenz der Organisation sichern (4.3.2). So haben insbesondere interessenbasierte Freiwilligenorganisationen - wie etwa die großen islamischen Dachverbände - nicht nur Erwartungen an die Mitglieder zu stellen, sondern ebenfalls deren Ansprüchen und Forderungen nachzukommen (Schmitter/Streeck 1999/[1981]: 19; Pries 2010a).

\section{Unterschiedliche Mitgliedertypen}

Demnach haben freiwillige Vereinigungen einen Schwerpunkt auf „motivierende Funktionen"zu legen (Horch 1983: 31, 56; 4.3.1), und zwar für unterschiedliche Mitgliedertypen, die sich z. B. in bezahlte Hauptamtliche, unbezahlte Ehrenamtliche,

einer Veränderung dieser spezifischen Ziele, für die die Mitglieder ihren ganzen Einsatz geben (Luhmann 1964: 100, Fn 21). 
unbezahlte Engagierte oder passive Mitglieder einteilen lassen ${ }^{41}$. Deren emotionale wie organisationale Einbindung ist als Herausforderung zu begreifen und ist für die Fortexistenz der Organisation von allergrößter Wichtigkeit (ebd.: 30 ff.; Kühl 2014: 345). In freiwilligen Vereinigungen und Arbeitsorganisationen verhält es sich mit der Motivation zu einer Mitgliedschaft, Teilnahme und Leistungserbringung also unterschiedlich: In ersteren liegt der Bruch nicht wie in letzteren „Zwischen Teilnahme und Leistungsmotivation, sondern zwischen Mitgliedschaftsund Teilnahmemotivation" (Horch 1983: 35).

Obgleich mit der freiwilligen Mitgliedschaft in Vereinen auf damit verbundene geringere Anforderungen geschlossen werden kann, sind mit der Übernahme eines Engagements oder Ehrenamtes natürlich auch Erwartungen und Verpflichtungen verbunden, wie etwa die verlässliche Übernahme bestimmter Aufgaben, Präsenz und Pünktlichkeit, sodass diesbezüglich auch von einer „Mitarbeiterrolle“ gesprochen werden kann (ebd.: 63).

Der Umfang und die Intensität einer aktiven Beteiligung der Mitglieder am Geschehen in einer Organisation kann sehr unterschiedlich ausfallen, weshalb die Entscheidung über den Status eines Mitglieds oder anderweitig Beteiligter bisweilen schwierig ist (s. dazu auch Fußnote 41). Letzteres trifft insbesondere auf die gemäß dem deutschen Vereinsrecht organisierten Moscheevereine zu, die sich vom deutschen Idealverein insofern unterscheiden als in ihnen ein grundsätzlich anderes Verhältnis zwischen Mitglieder- und Beteiligungsstruktur vorherrscht. So gibt es generell nur eine kleine Anzahl eingetragener beitragszahlender und über die satzungsgemäßen Pflichten und Rechte verfügenden Mitglieder, aber eine weitaus größere Anzahl von Moscheebesuchern, die Nicht-Mitglieder sind, die am Vereinsleben aber aktiv teilnehmen und sich einer Moschee zugehörig fühlen, ohne sich dort offiziell am Willensbildungsprozess zu beteiligen (Neumann 2004: 3). In einem ,deutschen' Verein bestehen für Nicht-Mitglieder hingegen offiziell keine Beteiligungsmöglichkeiten. Daher ist es in formaler Hinsicht zumeist schwierig, zuverlässige Angaben zu den Mitgliederzahlen von Moscheevereinen

\footnotetext{
${ }^{41}$ Heinz-Dieter Horch differenziert zwischen folgenden Mitgliedertypen: unbezahlten und bezahlten Mitarbeitern sowie Nicht-Mitarbeitern. Seine Mitgliedertypologie basiert auf fünf Kriterien:

1.) (Nicht-)Anwesenheit; 2.) (Nicht-)Teilnahme an der Vereinsarbeit und an Entscheidungsprozessen; 3.) (Nicht-) Übernahme von einzelnen Aufgaben und (un-)bezahlten Ämtern; 4.) Art des Outputs der Organisation für das Mitglied, d. h. Art der Anreize, die zur Mitwirkung führen; 5.) Input als Art des Beitrags bzw. der spezifischen Aufgaben durch das Individuum für die Vereinigung (z. B. Vorstandsarbeit) (Horch 1983: 35 f.; vgl. auch Mayntz 1963: 46; Etzioni 1975: 17-22).
} 
zu machen, und es wird zu Recht darauf hingewiesen, dass die Grundbestimmungen des deutschen Vereinswesens nicht dem Selbstverständnis der Muslime entsprechen (Lemmen 2002: $27 \mathrm{ff}$.).

Das Konzept von Mitgliedschaft bzw. die Zählung von Mitgliedern ist jedoch nicht nur in islamischen, sondern auch in migrantischen Vereinigungen im Allgemeinen eher flexibel ausgestaltet. Von einem einheitlichen Verständnis von Mitgliedschaft ist nicht auszugehen, denn neben formalen Mitgliedern mit einem offiziellen (dauerhaften) Mitgliedschaftsstatus werden häufig auch (un-)regelmäßig Engagierte und Nutzer, denen die Angebote der MSO offenstehen, zu den Mitgliedern gezählt. Eine einheitliche (messgenaue) Angabe von Mitgliedern seitens der MSO ist folglich nicht zu erwarten (vgl. auch Friedrichs et al. 2020: 20; III. 7.1.2.1).

Im Kontext der Mitgliedschafts- und Beteiligungsfrage macht Niklas Luhmann auf die für religiöse Organisationen charakteristische Unterscheidung von durchaus unterschiedlichen Mitgliedschaftskreisen bzw. -segmenten aufmerksam, die eine Formalisierung der Mitgliedschaftsrolle, d. h. ein spezifischen Erwartungen entsprechendes Verhalten nicht für alle Mitglieder einer religiösen Organisation bewirken kann. In Bezug auf große volkskirchliche Organisationssysteme unterscheidet Luhmann zwischen drei Mitgliedschaftstypen: 1.) hauptamtlich tätigen und bezahlten Amtsträgern, 2.) in der Organisation aktiven und präsenten Mitgliedern sowie 3.) nur rechnerisch relevanten, kirchensteuerzahlenden, innerhalb der Organisation aber passiv bleibenden Personen (Luhmann 1977: 298 ff.). Angesichts dieser Konstellation sind von der Kirchenleitung getroffene Entscheidungen nicht ohne Weiteres auf die der Kirchenmitglieder zu beziehen. Dadurch wird der Modus der „durchgehende[n] Entscheidungsverknüpfung“ als klassisches Merkmal für Organisationssysteme ausgehebelt (ebd.: 295).

Nach den Reflexionen über die Verantwortlichkeiten und Herausforderungen, die sich mit der Mitgliedschaft in einer Vereinigung ergeben, soll sich nun den individuellen Eigenschaften der Mitglieder und Mitwirkenden gewidmet werden. Dazu gehören neben soziodemografischen Merkmalen wie Alter, Geschlecht oder Ethnie natürlich auch sozioökonomische wie das Einkommen und das Bildungsniveau, die die Mitglieder befähigen, sich in einem bestimmten Ausmaß und mit spezifischen Fähigkeiten und Wissensbeständen in die Organisation einzubringen (Scott/Davis 2007: 24; Pugh et al. 1963: 312), und die damit dazu beitragen, dass diese bestimmte Angebote und Dienstleistungen unterbreiten kann (Halm et al. 2012a: 93; Halm/Sauer 2015: 59, 62, 80 f.).

In den folgenden zwei Unterkapiteln sollen diese Personenmerkmale und weitere Ressourcen (4.3.3.1) sowie individuelle Wertorientierungen und Motive (4.3.3.2), in ihrer Verbindung mit durch freiwillige Organisationen vorgehaltenen 
Gelegenheitsstrukturen, als engagementbedingende Aspekte näher ausgeleuchtet werden.

\subsubsection{Zur Relevanz von Personenmerkmalen und Netzwerken für Engagement}

Zivilgesellschaftliche Beteiligung muss man sich leisten können. Sie ist bedingt durch individuelle und soziale Ressourcen bzw. Netzwerke und ein soziales Eingebundensein. Diese Aspekte üben jeweils einen eigenständigen Einfluss aus, wirken aber auch wechselseitig aufeinander ein. Grundsätzlich ist menschliches Handeln auf Personenmerkmale, verfügbare Ressourcen, individuelle Dispositionen sowie lebensweltliche Gelegenheitsstrukturen zurückzuführen. Wenngleich diese einzelnen Dimensionen im Rahmen dieser Arbeit nicht empirisch untersucht werden können, sollen sie in ihren Zusammenhängen und Auswirkungen auf Engagementbeteiligung in diesem Kapitel näher beleuchtet werden. Herausgestellt wird die Existenz unterschiedlicher - ungleicher - Voraussetzungen: Ein Leben in finanziell und sozial gesicherten Verhältnissen bietet größere Spielräume, um (im Organisationskontext) auch für andere Menschen aktiv zu werden.

Empirische Untersuchungen zeigen deutlich, dass sich Personen mit einem hohen sozioökonomischen Status insgesamt stärker engagieren als Personen mit einer schlechteren Ressourcenausstattung (Wilson/Musick 1997, 1998; Wilson 2000; Schmidt 2014; Gensicke 2015; Simonson/Hameister 2016; BMFSFJ 2017: 156 ff.). Zu den sozioökonomischen Personenmerkmalen, die die Bereitschaft, sich zu engagieren, mitbestimmen, gehören die schulische und berufliche Bildung, das Einkommen bzw. die finanzielle Situation, der Erwerbsstatus ${ }^{42}$ sowie die Haushaltskonstellation. Von diesen stellen das Bildungs- und Einkommensniveau die zentralen Erklärungsfaktoren für die Aufnahme eines Engagements dar.

Mit unterschiedlichen sozioökonomischen Status gehen folglich disparate Zugangschancen zum zivilgesellschaftlichen Engagement einher. Der Freiwilligensurvey identifiziert eine kumulative Wirkung von sozioökonomischen Faktoren auf das freiwillige Engagement: Wenn vorteilhafte Ressourcen gleichzeitig gegeben sind, erhöht sich die Wahrscheinlichkeit eines freiwilligen Engagements. Diese sinkt hingegen beispielsweise bei einem niedrigen Bildungsniveau und Arbeitslosigkeit (Simonson/Hameister 2016: 429).

\footnotetext{
42 Bei Erwerbstätigen handelt es sich um Personen, die in einem Arbeitsverhältnis stehen, die selbstständig sind oder die einen freien Beruf ausüben.
} 
Anzunehmen ist, dass eine qua Bildungs- und Einkommensniveau gegebene gute Ressourcenausstattung eher mit engagementförderlichen Orientierungen und Wertvorstellungen einhergeht. Wer aufgrund von Eifer und Neugierde nach Informationen zu Engagementmöglichkeiten sucht, wird diese leicht finden. Zudem ist die Wahrscheinlichkeit höher, dass eine Person, die über bestimmte Qualifikationen und Kompetenzen verfügt, häufiger direkt angesprochen und öfter mit der Frage konfrontiert wird, ob sie ein bestimmtes anspruchsvolleres Ehrenamt oder Engagement übernehmen möchte (Müller et al. 2016: 408 f.; Simonson/Hameister 2016).

Es ist nachvollziehbar, dass finanzielle Absicherung Engagement begünstigt und dass sich Personen, die in Lohn und Brot stehen, anteilig häufiger engagieren als Erwerbslose (BMFSFJ 2017: 157). Eine schwierige oder unsichere finanzielle Lage dürfte betroffene Personen von einem Engagement abhalten, da existenzielle Fragen bewältigt werden müssen und die Suche nach einem finanziellen Auskommen Priorität hat. Im Rahmen einer freiwilligen Tätigkeit dürften notwendige Kostenauslagen (wie z. B. Fahrkarten und vereinsrelevante Einkäufe) ein Problem darstellen. Darüber hinaus ist der Erwerbsstatus bzw. ein Arbeitsverhältnis auch relevant aufgrund dadurch gegebener regelmäßiger Kontakte zu anderen Personen und damit vorhandenen leichteren Zugängen zu Informationen über Engagementmöglichkeiten.

Nicht nur soziale, sondern auch politische Beteiligung ist grundsätzlich abhängig vom sozioökonomischen Status, v. a. dem Bildungs- und Einkommensniveau. Ebenfalls bedeutsam sind das Vorhandensein von Opportunitäten, der Zugang zu sozialen Netzwerken, psychologische Dispositionen für die Auseinandersetzung mit Politik und nicht zuletzt verfügbare Zeitressourcen (Verba et al. 1995: 267 ff., 319 f.; Brady et al. 1995). Neben dieser Abhängigkeit des politischen Engagements von personenbezogenen Merkmalen wurden in der Forschung hinsichtlich der politischen Teilhabe von Menschen mit Migrationshintergrund im aufnahmegesellschaftlichen Kontext folgende wesentliche Einflussfaktoren identifiziert: 1.) die Aufenthaltsdauer im Aufnahmeland, 2.) der Umfang der dort in Abhängigkeit vom Staatsangehörigkeitsgesetz bestehenden politischen Rechte und Angebotsstrukturen sowie 3.) die politische Prägung des Herkunftslandes (Müssig/Worbs 2012; vgl. auch BMFSFJ 2017: 207; 3.2).

Vor diesem Hintergrund wird die geringere Engagementbeteiligung von Menschen mit einer Zuwanderungsgeschichte auch damit erklärt, dass engagementbegünstigende Bedingungen, die v. a. sozioökonomische Faktoren betreffen, bei ihnen seltener vorliegen als in der autochthonen deutschen Bevölkerung (BMFSFJ 2017: 205, 208 f.). Mit dem Aspekt einer schichtbedingten ungleichen Engagementbeteiligung unterschiedlicher Bevölkerungsgruppen ist die Frage nach 
vorteilhafteren Lebens- und Verwirklichungschancen für sozial besser gestellte Gruppen und damit auch die Frage der sozialen Ungleichheit angesprochen.

Die sozioökonomischen Faktoren sind als miteinander verknüpft zu betrachten. So ist etwa eine spezifische finanzielle Situation auf den Erwerbsstatus und den Bildungsgrad zurückzuführen und die Frage nach dem finanziellen Auskommen hängt mit der Versorgung einer bestimmten Anzahl von Haushaltsmitgliedern, der Haushaltskonstellation, zusammen (Simonson/Hameister 2016: 431). Aus der Wechselbeziehung von Haushaltskonstellation, Familie und Erwerbsleben ergibt sich eine Summe verfügbarer Zeit als eine wertvolle Ressource und Voraussetzung für freiwilliges Engagement (Zierau 2001).

In Familien kann über Angehörige eine Engagementbeteiligung stimuliert werden. Insbesondere beim Vorhandensein von Kindern können sich durch deren Eingebundensein in Schule, Kindergarten oder Vereine günstige Anlässe für die Aufnahme eines Engagements oder Ehrenamts durch die direkte Ansprache von Eltern ergeben (Müller et al. 2016: 408; Simonson/Hameister 2016: 429; Wilson/Musick 1997: 699; Smith 1994: 250). ${ }^{43}$

Auch die soziale Einbettung einer Person bzw. ihre Zugehörigkeit zu einer bestimmten Bevölkerungsgruppe wird wiederum von sozialstrukturellen Merkmalen wie dem Bildungsgrad, dem Geschlecht und auch dem Alter bestimmt. Diese Merkmale führen in der Summe zu ungleichen Ressourcenausstattungen, die das Engagementverhalten beeinflussen. Höher gebildete Menschen verkehren in anderen Milieus und Kreisen als Niedriggebildete, womit disparate Gelegenheitsstrukturen und Anstoßmöglichkeiten einhergehen (Müller et al. 2016: 408 f.). Ein zentraler Befund des Freiwilligensurveys ist, dass sich Personen, die sozial gut eingebettet bzw. integriert sind, häufiger engagieren als Menschen, bei denen dies nicht zutrifft. Das Phänomen der sozialen Einbettung oder Integration bezieht sich auf die Existenz sozialer Kontakte, Netzwerke und in deren Rahmen stattfindender Interaktionen. Der Freiwilligensurvey kommt zu dem Ergebnis, dass „,[e]ine gute Netzwerkqualität, das Vorhandensein sozialer Unterstützung außerhalb des

\footnotetext{
43 An dieser Stelle ist auf geschlechtsspezifische Unterschiede hinzuweisen: Wenngleich sich die Engagementbeteiligung zwischen Männern und Frauen in den letzten Jahren stark angenähert hat, fällt sie bei Frauen immer noch geringer aus. Die Differenzen im Engagement sind einerseits auf den unterschiedlichen sozioökonomischen Status (z. B. materielle Sicherheit) der Geschlechter zurückzuführen. Andererseits können sie aber auch durch die traditionelle geschlechtsspezifische Arbeitsteilung erklärt werden. Denn Frauen sind trotz einer stärkeren Erwerbsbeteiligung und höherem Bildungsniveau nach wie vor stärker für Haushalt und Kinderbetreuung zuständig als Männer und dürften daher über weniger zeitliche Ressourcen für eine freiwillige Tätigkeit verfügen (Vogel et al. 2016c: 623 ff.; BMFSFJ 2017: 144 ff.). Für Frauen ist die Vereinbarkeitsfrage eine existenzielle, d. h. eine Frage, die Aufstieg und Karriere eher behindert als bei Männern (Alscher 2010).
} 
eigenen Haushaltes und ein hohes soziales Vertrauen [...] unabhängig und signifikant mit einer erhöhten Wahrscheinlichkeit einher[gehen], sich zu engagieren“ (Huxhold/Hameister 2016: 489). Zudem können die durch soziale Netzwerkbeziehungen zur Verfügung gestellten Ressourcen am produktivsten von Menschen mit einem hohen sozioökonomischen Status (Vermögen, Bildung) genutzt werden. Sozialkapital ist somit v. a. ein guter Prädiktor für das Engagement von Menschen mit einem höheren sozialen Status (Wilson/Musick 1998).

Direkte Anreize, Anstöße oder Aufforderungen zur Übernahme eines Engagements oder Ehrenamts bieten sich häufig aufgrund der Existenz individueller sozialer Netzwerke und den sich hier ergebenden sozialen Interaktionen. Diese umfassen Familienangehörige, Freunde, Nachbarn und Bekannte, unter denen sich auch organisationsleitende Personen befinden können, die auf Suche nach Engagierten und Ehrenämtlern sind (Müller et al. 2016: 407). Empirische Befunde weisen nach, dass nicht allein die Größße, d. h. die Personenanzahl des sozialen Netzwerkes, sondern seine Qualität mindestens ebenso wichtig ist. Wie im Kapitel zu den sozialkapitaltheoretischen Blickrichtungen verdeutlicht wurde (4.2), können starke soziale Netzwerke einen hohen Grad sozialer Unterstützungsleistungen und ein hohes Niveau an Vertrauen in die Netzwerkangehörigen, aber auch in andere Menschen sicherstellen und dadurch Engagementbereitschaft stimulieren (van Ingen/Bekkers 2013).

Der Begriff des sozialen Netzwerks als solcher verweist wiederum auf die Kapitaltheorie von Pierre Bourdieu (1983, 1987), die bereits im Kapitel zum Sozialkapital (4.2.1) vorgestellt wurde. Beim Bourdieuschen Sozialkapital handelt es sich um ein Gut, das den Beziehungen zwischen Personen innewohnt, wobei sich das Reservoir an Ressourcen bei Bedarf für individuelles oder kollektives Handeln (zu persönlichen Gunsten) nutzen lässt. Bourdieus Überlegungen kann entnommen werden, dass in sozialen Netzwerken die Zugehörigkeit zu einer bestimmten Gruppe den eigentlich produktiven ,Mehrwert' darstellt. Die Sicherung einer dauerhaften Zugehörigkeit zu einem Netzwerk oder einer Gruppe und deren Fortbestand erfordert Bourdieu zufolge (Bourdieu 1983: 192) jedoch eine seitens des Gruppenmitglieds zu leistende „Institutionalisierungsarbeit“. Diese ist „für die Produktion und Reproduktion der dauerhaften nützlichen Verbindungen“ und den „Zugang zu materiellen oder symbolischen Profiten“ notwendig (ebd.: 192). Sie impliziert die Investition von Zeit und Geld und bestimmte Verpflichtungen, wie z. B. die Übernahme eines Ehrenamtes. Mit Blick auf die Ausstattung einer Person mit kulturellem, ökonomischem und sozialem Kapital stellt Bourdieu ihre wechselseitige Beeinflussung und Transformierbarkeit fest. Ein hohes kulturelles Kapital, das sich z. B. durch den Erwerb eines Hochschulabschlusses angeeignet wurde, kann eine gut dotierte Anstellung zur Folge haben und somit in 
ökonomisches Kapital transformiert werden (ebd.: 184 ff.). Bourdieus Annahmen korrespondieren somit mit den zuvor dargelegten Befunden der Engagementforschung, wie sie v. a. durch den Freiwilligensurvey ermittelt wurden, nämlich den wechselseitigen und kumulativen Wirkungen sozioökonomischer Faktoren auf Engagementbeteiligung.

Das kulturelle oder Bildungskapital korrespondiert mit dem Begriff des religiösen Kapitals (nicht: religiöses Sozialkapital), über das Individuen verfügen können. Religiöses Kapital kann ,als akkumulierte Arbeit im religiösen Feld“ (Foroutan 2017: 272, Fn 5) definiert werden. Es umfasst u. a. inkorporiertes Wissen und Bildung über eine spezifische Religion. Laurence R. Iannaccone (1990: 299) definiert religiöses Kapital als die ,familiarity with a religion's doctrines, rituals, traditions, and members", die die individuelle Zufriedenheit des Individuums durch dessen Teilnahme an der Religion und damit auch die Wahrscheinlichkeit seiner religiösen Partizipation steigert. Dies impliziert, dass Religiosität bzw. religiöse Werte mit entsprechenden religiösen Einstellungen und gemeinschaftsbezogenen Verhaltensweisen einhergehen. Insofern ist religiöses Kapital als eine Komponente von sozialem Kapital zu betrachten (vgl. 4.2.4). Iannaccone beschreibt religiöses Kapital als eine Voraussetzung wie auch Folge von religiösen Aktivitäten gleichermaßen. ${ }^{44}$

Die Berücksichtigung von religiösem Kapital ist insofern relevant, als dass angenommen werden kann, dass eine hohe subjektive Religiosität positiv mit freiwilligem Engagement korreliert ist (4.2.4; 4.4.2). In organisationssoziologischer Hinsicht ist aufschlussreich, dass Ergebnissen internationaler Studien zufolge häufig die Kirchengangshäufigkeit - als Indikator für religiöse Einbindung - ein starker Prädiktor für freiwilliges Engagement ist. Hingegen weisen der Gottesglaube und die Konfessionszugehörigkeit allein gar keinen bzw. nur einen schwachen Zusammenhang mit freiwilliger Arbeit auf. Religiosität und die Mitgliedschaft in einer religiösen Gemeinde in Kombination mit der Häufigkeit von Gottesdienstbesuchen haben - vereinfacht zusammengefasst - einen signifikant positiven Effekt auf freiwilliges Engagement (Berger 2006; Yeung 2004; Becker/Dhingra 2001; Ruiter/De Graaf 2006; Hoge/Yang 1994). Als engagementauslösend erweist sich also nicht zuvorderst die subjektive Religiosität, sondern eher die Handlungsebene

\footnotetext{
${ }^{44}$ Die Erweiterung des Kulturkapitalbegriffs von Bourdieu um religiöse und andere Werte geht auf Kritik an Bourdieus Konzeptualisierung zurück, die das Kognitive überbetone und moralische Komponenten von Kultur vernachlässige (Wilson/Musick 1997: 696). So fehlten in der Analyse Bourdieus moralische Bezüge auf Ehrlichkeit, Verantwortlichkeit, Vertrauenswürdigkeit, Fairness, Friedfertigkeit etc. (ebd., unter Verweis auf Lamont 1992: 185); diese Dimensionen würden allerdings auch sozialisiert, verinnerlicht und in sozialen Interaktionen benötigt.
} 
des Kirchgangs („behaving“), die „'institutional’ channels“(Wilson/Musick 1997: 710). Robert Wuthnow führt in diesem Zusammenhang erläuternd aus, dass ,religious organizations tell people of opportunities to serve, $[\ldots]$ and provide personal contacts, committees, phone numbers, meeting spaces, transportation, or whatever it may take to help turn good intentions into action“ (Wuthnow 1994: 253).

\subsubsection{Motive freiwilligen Engagements}

Im alltagssprachlichen Gebrauch bezeichnet ein „Motiv“ einen konkreten Beweggrund oder Auslöser für subjektives Handeln. In diesem Kapitel soll der Frage nachgegangen werden, welche Prozesse und Mechanismen im Kontext subjektiver Motive und der Wahl eines Handelns - bzw. der Entscheidung für ein Engagement in einer Organisation - relevant sind und wie sich Motive in der empirischen Engagementforschung konkret fassen und benennen lassen. Angeknüpft wird dabei zunächst an die bereits dargelegten Überlegungen Niklas Luhmanns zu Motivationsfragen innerhalb von Arbeitsorganisationen, die mit Blick auf freiwillige, nicht gewinnorientierte Vereinigungen kaum Gültigkeit beanspruchen können. Im Anschluss sollen handlungsauslösende Prozesse aus motivationspsychologischer und soziologischer Perspektive beleuchtet werden. Dabei werden Präferenzen berücksichtigt, die intrinsische (auch uneigennützige) Motive von Individuen und damit auch nicht greifbare Interessen, wie etwa Gerechtigkeitsfragen und soziale Anerkennung, einkalkulieren. Die Darlegung der zentralen theoretischen Prämissen zeigt, dass menschliche Motive und Handlungen immer im Kontext von subjektiv wahrgenommenen bzw. definierten Situationsumständen, der individuellen Lebenssituation, von Persönlichkeitsmerkmalen und -dispositionen, verfügbaren Ressourcen sowie vorhandenen Opportunitäts- und Gelegenheitsstrukturen zu betrachten sind, wobei letztere insbesondere auch durch Organisationen offeriert werden.

Luhmann zufolge wird durch die Separation von Teilnahmemotivation und Leistungsmotivation sowie die Trennung von Motiven und Organisationszweck in intern stark differenzierten Organisationen gewährleistet, dass letztere sich flexibel an Veränderungen in ihrer Umwelt anpassen können, ohne dass dies Rückwirkungen auf den Bestand der Mitglieder und deren Motivationsstrukturen hat (4.3.3). Die Separation von Motivationsfragen ist allerdings auf Arbeitsorganisationen und deren Vorteil von „bezahlter Indifferenz“ (Luhmann 1964: 96) einzugrenzen. Diese „,Kapitalisierung “ der Motivation“ gewährleiste innerhalb gewisser Grenzen die Unterwerfung des Mitglieds unter die Formalstruktur der Organisation und die Unabhängigkeit von Motivations- und Zweckstruktur, die damit variabel 
und anpassbar bleiben (ebd.: 101). Luhmann selbst betrachtet die Mitgliedschaftsrolle als „Abstraktion eines Motivationspotenzials“ (Luhmann 2000: 84), die so nicht auf die in Vereinen vorgefundenen Verhältnisse zu übertragen ist. Er selbst spricht jedoch auch die soziale Natur starker Motivkräfte an, die sich aus persönlichen Wunschvorstellungen, Anspruchsniveaus, emotional-geselligen Aspekten, gemeinsamen Wertschätzungen, dem Ausmaß an Selbstkontrolle, dem Gewinn von sozialen Status, dem Prestige der Organisation und Situationsvergleichen ergeben (Luhmann 1964: 89 f.). Damit geraten individuelle, extraorganisationale Wünsche, Ziele, Motive und Prozesse in den Blick, die das Individuum dazu bringen, sich für ein Engagement in einer Organisation zu entscheiden, und die in kollektivistischen Ansätzen der Systemtheorie oder des Funktionalismus weitgehend ausgeblendet werden (Opp 2014: 375). Diese Motive sind zusammenzudenken mit Anreizen, die die Organisation bieten muss, allerdings nicht deckungsgleich mit solchen (4.4), da sie v. a. auf spezifische Personenmerkmale zurïckzuführen sind.

In der Motivationspsychologie werden unter Motiven individuelle, fortwirkende Wertungsdispositionen verstanden, die menschliches Handeln beeinflussen und die sich im Sozialisationsprozess entwickeln (Heckmann 1989: 10). Motive lassen sich allgemeinen Handlungszielen wie „Leistung“, Hilfeleistung“, ,,intellektuelle Tüchtigkeit“, „,sozialer Anschluss“, „,Macht“ zuordnen (ebd.: 2, 16). Die Motivationsforschung fragt nach den mit einer konkreten Aktivität angestrebten Zielen, nach dem „Wozu“ und damit nach der Zweckrationalität einer Handlung (ebd.: 1 f., 189). Mit dem eigenen Handeln sollen antizipierte wünschenswerte und angestrebte Folgen und Erwartungen herbeigeführt werden (ebd.: 11 f., 189).

Als ein grundlegendes handlungsleitendes Prinzip fungieren Werte oder Wertorientierungen. Werte sind als sinngebende Grundprinzipien, allgemeine Orientierungsstandards oder Präferenzkriterien zu verstehen (Korte/Schäfers 2016: 39 f.). Sie umfassen Vorstellungen vom Wünschens- oder Erstrebenswerten, von nutzenversprechenden oder -stiftenden Gütern und enthalten als ethische Imperative damit immer auch eine normative Komponente (ebd.), die das Handeln des Menschen allgemein abstecken und unterschiedlich stark strukturieren können $^{45}$ (Schwartz/Bilsky 1987, 1990; Welzel 2009). Im Gegensatz zu Einstellungen oder Meinungen weisen Werte einen stabilen, dauerhaften Charakter auf (Pickel

\footnotetext{
45 Zur Erfassung von grundlegenden Werten wird häufig auf das prominente ,Wertekreismodell" des Sozialpsychologen Shalom Schwartz zurückgegriffen. Es umfasst zehn Wertorientierungen, die in zwei Spannungsachsen zwischen Selbstentfaltung und Bewahrung/Anpassung einerseits sowie Selbstbezogenheit und Fremdbezogenheit/Altruismus andererseits zu verorten sind. (Die zehn Werte werden wie folgt benannt: Anpassung, Genuss, Kreativität, Macht, Ökologie, Wohlstand, Sicherheit, Prosozialität, Stimulation, Tradition) (Pickel 2018: 960 f.).
} 
2018: 959). Sie werden im Sozialisationsprozess durch verschiedene Sozialisationsinstanzen wie den Eltern, der Peergroup oder der Schule vermittelt und internalisiert. Im Lebensverlauf werden sie häufig verfestigt und besitzen eine anhaltende Prägekraft für die subjektive Lebensgestaltung (ebd.: 957, 959). In Abhängigkeit von sozialstrukturellen Faktoren und subjektiven Erfahrungen können sie sich im Zeitverlauf aber auch verändern oder an Bedeutung verlieren. Einzelne Werte stehen jedoch nicht isoliert nebeneinander, sondern korrespondieren miteinander, wie z. B. die Werthaltungen Sicherheit und Tradition; andere scheinen sich hingegen eher abzustoßen und weniger kompatibel zu sein, wie etwa die Werte Tradition und Genuss bzw. Hedonismus. Zudem können Wertorientierungen die Aufnahme und Beibehaltung eines Engagements stimulieren, diesem aber auch entgegenstehen; demnach lassen sie sich als engagementförderliche oder -hinderliche einstufen. So zeigen Ergebnisse des Freiwilligensurveys, dass sich Menschen, denen die Werte Solidarität und Kreativität wichtig sind, anteilig häufiger engagieren als Menschen, denen diese Werte weniger wichtig sind; Menschen, denen der Wert Sicherheit wichtig ist, engagieren sich anteilig seltener als Personen, die diesen Wert für nicht so bedeutsam halten (Huxhold/Müller 2016: 475).

Wenn Werten in konkreten Situationen handlungsleitende Relevanz zukommt, kann von „Motivationen“ oder „Bedürfnissen“ gesprochen werden (Gensicke/Neumaier 2014: 610). Ihre Verhaltenswirksamkeit entfalten Werte in konkreten alltäglichen (Entscheidungs-)Situationen durch ihre Operationalisierung in sozialen Normen (Korte/Schäfers 2016: 39). Diese stellen im Sozialisationsprozess vermittelte und verbindlich gemachte Verhaltensregeln oder gesellschaftlich überwachte Sollenserwartungen dar, die Kategorien von ,gut“ und „schlecht“, Verbote und Erlaubtes festsetzen und das subjektive Handeln steuern (Joas 1999: 267). Durch ihre Standardisierung ermöglichen soziale Normen Handlungswiederholungen und stiften in Interaktionen wechselseitige Erwartungssicherheit. Wie im vorigen Kapitel verdeutlicht wurde, sind subjektive Wertungsdispositionen und die mit einem Handeln bzw. Engagement verknüpfte Erwartung dabei immer von den Fähigkeiten der handelnden Person und den ihr zur Verfügung stehenden Ressourcen wie Geld, Bildung und Zeit abhängig (Wilson/Musick 1997, 1998; Simonson/Hameister 2016; Müller et al. 2016). Mit Blick auf freiwillige Unterstützungsleistungen sind daher für unterschiedliche Bevölkerungsgruppen verschiedene Motive bedeutsam. So zeigen die Befunde des Freiwilligensurveys 2014, dass insbesondere hochgebildete Personen durch ihr Engagement die Gesellschaft mitgestalten wollen bzw. sich zutrauen, etwas bewirken zu können. Für Niedriggebildete ist hingegen der soziale, gesellige Aspekt im Rahmen ihrer 
Engagementbeteiligung wichtig, was generell aber auch für alle Bildungsgruppen zutrifft (Müller et al. 2016: 422, 425; Böhnke/Dathe 2010).

Ferner sind bei der Erklärung der Ursachen menschlichen Verhaltens neben den Personenfaktoren situative Gegebenheiten zu berücksichtigen. Aus den Wechselwirkungen innerhalb einer „Person-Situations-Interaktion“ resultiert eine spezifische situationsbedingte, handlungszielgerichtete „Motivation“ oder „Motivationstendenz" (Heckhausen 1989: 11). Bei der Motivation ist aufgrund der Person-Situations-Interaktion von einem Prozess zu sprechen: Eine konkrete Situation kann für ein Individuum einen Anreiz mit Aufforderungscharakter ${ }^{46}$ darstellen, den Eintritt von antizipierten erwünschten oder erwarteten Folgen des eigenen Handelns als wahrscheinlich zu erachten. Da häufig mehrere Motivationstendenzen nebeneinander bestehen, muss in einem Prozess ${ }^{47}$ entschieden werden, welche von ihnen in konkretes Handeln umgesetzt werden sollen. Das betrifft zumeist die am stärksten ausgeprägtesten (ebd.: 12 f.). In der Psychologie bezeichnet der Motivationsbegriff somit komprimiert „vielerlei Prozesse und Effekte, deren gemeinsamer Kern darin besteht, daß ein Lebewesen sein Verhalten um der erwarteten Folgen auswählt und hinsichtlich Richtung und Energieaufwand steuert" (ebd.: 10).

Diese Vorgänge und bezweckten Wirkungen korrespondieren mit dem interpretativen soziologischen Paradigma, das davon ausgeht, dass jede Handlungsentscheidung grundsätzlich zuvor des Vorgangs der subjektiven Definition der Situation bedarf, in deren Rahmen die individuellen Präferenzen und Motive bzw. Erwartungen immer wieder neu sowohl kognitiv als auch emotional strukturiert werden. Beim Motivationsprozess handelt es sich also um einen kognitiven Verarbeitungsprozess, bei dem auch Emotionen eine Rolle spielen können. Zentral sind dabei die subjektiven und damit möglicherweise auch falschen Wahrnehmungen und Vorstellungen des Individuums, die nicht zwangsläufig mit den objektiven Gegebenheiten übereinstimmen (Esser 1996: 2 f.; Opp 2014: 375 f.). Diese Annahme wird im prominenten Thomas-Theorem pointiert: „If men define situations as real, they are real in their consequences" (Thomas/Thomas 1928: 572). Das Thomas-Theorem korrespondiert mit Max Webers Konzept des subjektiven Sinns des menschlichen Handelns bzw. Verhaltens sowie seiner Definition des Motivs als einen Sinnzusammenhang, ,welcher dem Handelnden selbst oder

46 „Alles was Situationen an Positivem oder Negativem einem Individuum verheißen oder andeuten, wird als ,Anreiz" bezeichnet, der einen ,Aufforderungscharakter" zu einem entsprechenden Handeln hat“" (Heckhausen/Heckhausen 2006: 5).

${ }^{47}$ Der Prozess umfasst folgende Stadien bzw. Übergänge von der Motivation zum Handeln: 1. die Motivation, 2. die resultierende Motivationstendenz, 3. die Intentionsbildung, 4. die Handlungsinitiierung und 5. das Handeln (Heckhausen 1989: 13). 
dem Beobachtenden als sinnhafter, Grund ' eines Verhaltens erscheint ${ }^{\text {*48 }}$ (Weber 1985/[1922]: 5; Herv.: d. Verf.). Analog zum oben angeführten Prozess erfolgt nach Alfred Schütz die Entscheidung für eine Handlung nach der ,Tendenz des ,Wollens ““ bzw. der höchsten „Handlungstendenz“, da ,,jeder das ausführt, was er ausführen will, wenn er es nur ausführen kann“ (Schütz 1971a: 104). Die Definition der Situation durch die Auswahl spezifischer Aspekte vollzieht sich entlang des Hauptmotivs des persönlichen Interesses; die Selektion entspricht demnach einer „Motivationsrelevanz“ (Schütz 1971b: 78).

Im Rahmen der „Rational Choice“-Theorie (RCT) oder Theorie der rationalen Wahl gerät zielgerichtetes - d. h. vorausschauendes - menschliches Entscheidungsverhalten im Rahmen eines rational abwägenden Handlungsprinzips und in Anbetracht bestehender Handlungsbeschränkungen sowie konkurrierender Handlungsalternativen in den Blick. Einen zentralen Ausgangspunkt bildet dabei die Hypothese der Nutzenmaximierung, der zufolge Menschen im Sinne ihrer persönlichen Zufriedenstellung so handeln, dass sie ihre Ziele bestmöglich realisieren können. ${ }^{49}$ Mit einer liberalisierten Formulierung des Rationalitätsprinzips wird angenommen, dass Akteure versuchen, ihre Entscheidungen so auszurichten, dass in bestimmten Situationen, unter spezifischen Gegebenheiten (Anreizen), unter Berücksichtigung ihrer persönlichen Präferenzen (Motive, Ziele) und verfügbarer Mittel ein bestmöglicher Zustand erzielt wird (Braun 2009: 394, 399 f.). RCT-Soziologen plädieren dafür, alle Arten von Präferenzen und Restriktionen als Determinanten individuellen Handelns und damit auch existierende Normen und seitens Bezugspersonen bestehender Erwartungshaltungen zu berücksichtigen, da auch deren Erfüllung Befriedigung auslöst und damit Nutzen bringt (Opp 2014: 376). Hinsichtlich des Entscheidungsverhaltens werden folglich soziale

48 „,Handeln“ soll dabei ein menschliches Verhalten (einerlei ob äußeres oder innerliches Tun, Unterlassen oder Dulden) heißen, wenn und insofern als der oder die Handelnden mit ihm einen subjektiven Sinn verbinden“ (Weber 1985/[1922]: 1; Herv.: d. Verf.).

${ }^{49}$ Nach dem eng gefassten ökonomischen Konzept des neoklassischen Homo oeconomicus erfolgt die Selektion eines bestimmten Handelns nach dem Maßstab der Maximierung subjektiver Nutzenerwartung durch eine kontinuierliche Abwägung der durch unterschiedliche Handlungsmöglichkeiten entstehenden Kosten und Nutzen (Esser 1991: 431). Das Postulat der rationalen Wahl wird allerdings eingeschränkt durch das von Herbert Simon (1959, 1967, 1982a, b, 1990, 1997) ins Feld geführte Prinzip der begrenzten Rationalität (,,bounded rationality“): Aufgrund kognitiver Kapazitätsbeschränkungen können Informationen durch den Menschen nicht vollumfänglich aufgenommen und verarbeitet werden, sodass bei der Selektion einer Handlung vom Kriterium einer subjektiv erwogenen hinreichenden persönlichen Zufriedenstellung (,satisficing“) und nicht von einer Maximierung subjektiver Nutzenerwartungen (,maximizing“) ausgegangen wird (u. a. Simon 1959: 262 ff., 1967: 261; Riker/Ordeshook 1973: 21 ff.). 
Einflüsse und disponible Ressourcen wie z. B. der sozioökonomische Status einbezogen, ferner auch soziale Netzwerke, die Verfügbarkeit von Zeit, die Existenz einzuhaltender Normen (Gesetze) sowie institutionelle Regelungen (Braun 2009: 402 f.; Opp 2014: 375 f.). Bezüglich des Prozesses der Handlungswahl erfolgt im Rahmen der RCT eine Bewertung von allen möglichen Handlungsalternativen aufgrund subjektiver Präferenzen und der in Zusammenhang mit einer spezifischen Handlung vermuteten Wahrscheinlichkeit des Eintretens eines bestimmten Ereignisses (outcome).

Vor diesem Hintergrund wird menschliches Verhalten keinesfalls immer von einem individuellen Profitstreben angeleitet. Nach Hartmut Esser (1990) bestimmen kognitive Relevanzrahmen - „frames“ - und Routinen - „habits“ - die Orientierung des Handelns in subjektiv definierten Situationen und lassen sich als Spezialfälle der Theorie der rationalen Wahl konzipieren. Die oben thematisierte subjektive Definition der Situation erfolgt durch eine Vereinfachung komplexer Informationen und eine Akzentuierung der Situation qua Selektion eines dominierenden Rahmens (Framing). Die Annahme der rahmenden Situationsdefinition stellt eine Grunddoktrin der soziologischen Handlungstheorien dar (Esser 1996: 12). Sie gewährleistet eine Fokussierung auf ein übergreifendes, oberstes Ziel. Das die Situation dominierende „Leitmotiv“ des Handelns (Esser 1991: 438) und die situationsspezifisch erfolgte Einordnung der Zielerreichung bestimmen in einem zweiten Schritt die Selektion einer Handlung bzw. die Aktivierung einer Routine oder Gewohnheit (engl: „habit“) (Esser 1990: 234 f.). „Habits“ bezeichnen bestimmte routinisierte Handlungsweisen bzw. ,unreflektierte Reaktionen ohne Ziel-Mittel-Kalkulationen [...], die auf bestimmte Umgebungsreize hin ausgelöst werden“ (ebd.: 234). Sie folgen bestimmten kognitiv verankerten, häufig normativ gestützten und habituell gesicherten Wissensstrukturen - „Schemata“ oder „Skripten" -, die in typischen Situationen aktiviert werden. Diesbezüglich verwiesen werden kann auf den gesonderten Typus des traditionalen Handelns von Max Weber, das „durch eingelebte Gewohnheit“ (Weber 1985/[1922]: 12) erfolge und in alläglichen Handlungssituationen dominant vorkomme.

Habits und Frames vereinfachen Situationen und die Auswertung von Informationen und rationalisieren damit Entscheidungsprozesse. Der Rückgriff auf Routinen und Rezeptwissen im Alltag, die sich in ähnlichen Situationen bewährt haben, impliziert die Antizipation von Zielen und ist entsprechend als überlegt und vernünftig und nicht ,irrational“ einzustufen (Schütz 1972: 33 ff.; Esser 1990: 244, 1991: 439, 1996: 30 ff.). Analog zu dieser Argumentation lassen sich Esser zufolge (1990: 244, 1991: 442 f., 1996: 30) dann auch die vier bekannten Idealtypen sozialen Handelns von Max Weber (1985/[1922]: 12 f.) - das zweckrationale, 
das wertrationale, das traditionale und das affektuelle Handeln - als Randbedingungen einer Handlungssituation und spezifische Konstellationen von Frames, Habits oder einem „unbedingten Eigenwert“ (Weber 1985/[1922]: 12; Herv. i. Orig.) einer Verhaltensweise modellieren.

Weber selbst konstatiert, dass seine vier Idealtypen sozialen Handelns ${ }^{50}$ (bzw. von Motivlagen) selten in Reinform, sondern vielmehr in Mischformen auftreten. Nach Weber liegt sowohl beim affektuellen als auch beim wertrationalen Verhalten die Sinnhaftigkeit des Handelns in der Art des Handelns als solcher und ist nicht in einem Erfolgsstreben begründet (ebd.: 12 f.). Weber verneint die Sinnhaftigkeit und die Rationalität des wertrationalen, traditionalen und affektuellen (emotionalen) Verhaltens ab einer gewissen Stärke der Verhaltensorientierung an einer Reinform.

In der Betrachtungsweise der Weberschen Handlungstypen als Frames und im Kontext der Festlegung eines dominierenden und handlungsleitenden Motivs kann den Handlungstypen jeweils ein bestimmter Status zugeschrieben werden. Hartmut Esser (1991: 442) zufolge könnte das traditionale als ein Spezialfall von habituellem Handeln und die (Zweck-)Rationalität als ein Spezialfall des rationalen Handelns betrachtet werden.

Vor dem Hintergrund der obigen Darlegungen ist an dieser Stelle nochmals herauszustellen, dass Opportunitäts- bzw. Gelegenheitsstrukturen der Lebensumwelt konkrete persönliche Wertungsdispositionen und die Realisierung

${ }^{50}$ Gemäß der Weberschen Typologie sozialen Handelns (Weber 1985/[1922]: 12 f.; Herv. i. Orig.) handelt (1) zweckrational ,wer sein Handeln nach Zweck, Mittel und Nebenfolgen orientiert" und letztere dabei ,gegeneinander rational abwägt“. (2) Wertrationales Handeln äußere sich ,durch bewußten Glauben an den - ethischen, ästhetischen, religiösen oder wie immer sonst zu deutenden - unbedingten Eigenwert eines bestimmten Sichverhaltens rein als solchen und unabhängig vom Erfolg“. Unbeeinflusst von antizipierbaren Folgen gehe es um das Handeln im Dienst der persönlichen Überzeugung von der Wichtigkeit eines Anliegens, von religiösen Wertvorstellungen und Geboten oder von für sich selbst identifizierten Verpflichtungen. Je stärker das Verhalten wertrational orientiert sei bzw. bestimmte Werte - unbedingte Eigenwerte (z. B. absolute Güte, Pflichtmäßigkeit) - für das persönliche Aktivwerden absolut gesetzt würden, desto weniger würden Handlungsfolgen subjektiv reflektiert und desto, ,irrationaler" falle die Handlungsorientierung aus. (3) Traditionales Handeln erfolge ,durch eingelebte Gewohnheit“. Weber zufolge kommt das streng traditionale Verhalten in alltäglichen Handlungssituationen dominant vor und entspricht einer rein reaktiven Nachahmung, einem dumpfen reflexartigen Reagieren auf gewohnte Reize und sei häufig nicht als ,,sinnhaft“" orientiertes Handeln zu kategorisieren. Es ähnele damit dem (4) affektuellen (oder emotionalen) Verhalten, das ,durch aktuelle Affekte und Gefühlslagen“ evoziert und häufig ebenfalls nicht ,bewußt ,sinnhaft" orientiert ist“. Affektuelles Handelns zeige sich beispielsweise in Genussbefriedigung, Hingabe und (ungehemmtem) Abreagieren von Emotionen. 
oder Einschränkung bzw. Unterbindung von Handlungen beeinflussen. So lassen sich zum einen Handlungsunterschiede, z. B. zwischen Authochtonen und Allochthonen, auf in unterschiedlichem Ausmaß zur Verfügung stehende Ressourcen und Realisierungsmöglichkeiten zurückführen (4.3.3.1). Zum anderen bieten zivilgesellschaftliche Organisationen strukturelle Gelegenheiten für freiwilliges Engagement; sie werben aktiv um Freiwillige und gewinnen solche durch persönliche Ansprache (Müller et al. 2016; Simonson/Hameister 2016; 4.3.3.1). So kann z. B. ein Sportverein seinen Mitgliedern wichtige Dienstleistungen, soziale Unterstützung und Netzwerke sowie Opportunitätsstrukturen für eine freiwillige oder ehrenamtliche Tätigkeitsausübung bieten (Borggrefe et al. 2012). Die Anstöße und Anreize von außen müssen allerdings auf eine subjektive Motivlage stoßen, um wirksam zu sein. Hinsichtlich der konkreten Bereitschaft zu einer freiwilligen, unbezahlten Unterstützungsleistung ist also das Vorhandensein einer inneren engagementbegünstigenden Disposition - Motivlage - wichtig.

Motive können allgemein in antagonistische Kategorien eingeteilt werden, z. B. „,egoistisch“ und „,altruistisch“ oder ,intrinsisch“ und „extrinsisch“, also aus dem persönlichen Inneren kommend oder von außen gesteuert. Bei freiwillig Engagierten ist jedoch nicht von einem einzigen Motiv, sondern von einer Motivkombination auszugehen, z. B. weil sie anderen Menschen helfen, dabei zugleich aber auch Qualifikationen erwerben möchten. So wird in der Engagementforschung von „Motivbündeln“ gesprochen, die exemplarisch wie folgt benannt werden können (BMFSFJ 2017: 162; vgl. auch IfD Allensbach 2013: 35 f.):

- „Engagement, um Dinge zu bewegen und zu verbessern;

- Engagement aus Wertüberzeugungen und Altruismus;

- Engagement als Sinngebung des eigenen Lebens durch bedeutsame Aufgaben und Anerkennung;

- Engagement als Bereicherung des eigenen Lebens (etwa durch Geselligkeit);

- Engagement als Entfaltung von Fähigkeiten und Neigungen;

- Engagement, um Entscheidungsfreiheit zu haben;

- Engagement durch Anstöße von anderen (etwa um andere nicht zu enttäuschen);

- Engagement für einen konkreten Nutzen. “51

\footnotetext{
${ }^{51}$ Die sechs benannten „Motivbündel“ basieren auf einer mit 24 unterschiedlichen Engagementmotiven durchgeführten Explorativen Faktorenanalyse (EFA) (IfD Allensbach 2013: 34).
} 
Mit dem Erhebungsinstrument des Freiwilligensurveys 2014 werden konkret acht Motive abgefragt, die von den Befragten mit einer Fünfer-Skala einzustufen waren (Müller et al. 2016: 411):

- „Mein Engagement macht mir Spaß.

- Ich will vor allem mit anderen Menschen zusammenkommen.

- Ich will die Gesellschaft zumindest im Kleinen mitgestalten.

- Ich möchte mit Menschen anderer Generationen zusammensein.

- Ich will mir Qualifikationen erwerben, die im Leben wichtig sind.

- Ich will Ansehen und Einfluss in meinem Lebensumfeld gewinnen.

- Ich will durch mein Engagement auch beruflich vorankommen.

- Ich möchte mir durch das Engagement etwas dazuverdienen.“

Religiöse und migrationsspezifische Motive - wie den eigenen Landsleuten bei der Integration in Deutschland zu helfen, die Herkunftskultur in Deutschland aufrechtzuhalten, zur Verbesserung der Lebenssituation von Migranten in Deutschland beizutragen oder die Fortdauer der Bindungen der Landsleute an das Herkunftsland zu unterstützen (Halm/Sauer 2005: 140 ff.; Sauer 2011: 230 ff.) - werden nicht abgefragt. Diese sollten in einer Befragung von aus unterschiedlichen Ländern und Kulturen stammenden Menschen bzw. der von ihnen gegründeten Organisationen zwecks tiefergehender Erkenntnisse zu den Engagementausrichtungen jedoch berücksichtigt werden. Mit dem Engagement verknüpfte migrationsspezifische Erwartungen wurden im Rahmen der vorliegenden auf der Mesoebene angesiedelten empirischen Arbeit jedoch nicht als Motive der Engagierten, sondern als kollektive Ziele der migrantischen Vereinigungen erhoben (III. 6.1). Die Ziele fungieren allerdings wiederum als Indikatoren für die Motive der Engagierten, sich in den Organisationen einzubringen. Denn Organisationsziele und Motive sollten miteinander korrespondieren und es ist von einer Interdependenz bzw. Verquickung zwischen der Meso- und der Mikroebene und sich wechselseitig verstärkenden Phänomenen auszugehen (4.3.1).

Bestimmte religiöse Wertüberzeugungen, die in der Öffentlichkeit im Zusammenhang mit der Übernahme ehrenamtlicher Tätigkeiten und als eine selbstlose, uneigennützige - altruistische - Denk- und Handlungsweise thematisiert werden, stellen unter vielfältigen Motiven eine relevante Dimension dar. Dabei ist ein altruistisches Motiv theoretisch voraussetzungsvoller als es in der Engagementforschung angenommen wird, wenn konstatiert wird, dass in einem Engagement „Selbstlosigkeit und Eigennutz sich nicht ausschließen, sondern sinngebend miteinander verknüpft werden“ können (Müller et al. 2016: 410; s. auch Hacket/Mutz 
2002; BMFSFJ 2012; IfD Allensbach 2013). Denn altruistisches oder prosoziales Verhalten ist im strengen Sinn ausschließlich auf das Wohlergehen anderer Menschen gerichtet. Insofern ist zu diskutieren, ob und inwiefern der Aspekt der ausschließlichen selbstlosen Fremddienlichkeit insofern unrealistisch ist, als das Verhalten des Individuums immer auch dessen eigenen Interessen folgt, indem beispielsweise Bestrafungen oder negative Gefühlslagen vermieden oder den eigenen Wertanforderungen entsprochen und damit ein Zustand der individuellen Befriedigung ausgelöst wird (Heckhausen 1989: 282 ff.). Auch bei dem als Musterbeispiel für altruistisches Verhalten geltenden Gleichnis vom Barmherzigen Samariter (Lukas 10, 30-35 $5^{52}$ ) ist der Aspekt der Selbstdienlichkeit nicht auszuschließen. Das Handeln des Samariters erscheint grundsätzlich besonders selbstlos und seiner Person zuzuschreiben, da er Mühen und Kosten auf sich nimmt und keine Aussicht auf eine Gegenleistung hat. In der Einöde steht sein Handeln nicht unter Beobachtung und Kontrolle Dritter, wodurch er keine Sanktionen zu befürchten gehabt hätte, wenn er einfach weitergegangen wäre (Heckhausen 1989: 279, 285). Die Realisierung von als persönlich verbindlich betrachteten Werten und Normen durch die Hilfeleistung und die einhergehende persönliche Zufriedenstellung sind jedoch in diesem Fall dem Aspekt der Fremdbezogenheit unterzuordnen, weil für den Samariter keine extrinsische Belohnung oder Bestrafung zu erwarten war, womit es sich vornehmlich um eine altruistische Motivation gehandelt haben sollte. Vor diesem Hintergrund kann der Kompromiss formuliert werden, dass eine Handlung dann als altruistisch gelten kann, wenn der Aspekt der Fremdbezogenheit den übergeordneten Beweggrund darstellt (ebd.: 298, 301 ff.).

Prosoziales, altruistisches Verhalten oder Hilfehandeln ist an soziale Normen als allgemeine Verhaltensregeln gebunden, die etwa in einer Religion kodifiziert oder tradiert worden sind (z. B. „Liebe Deinen Nächsten wie Dich selbst“, Matthäus 19:19; „Der Prophet, Allahs Segen und Heil auf ihm, sagte: Keiner von euch darf sich als gläubig ansehen, bis er seinem Bruder oder seinem Nachbarn

52 „Ein Mann ging von Jerusalem nach Jericho hinab und wurde von Räubern überfallen. Sie plünderten ihn aus und schlugen ihn nieder; dann gingen sie weg und ließen ihn halb tot liegen. Zufällig kam ein Priester denselben Weg herab; er sah ihn und ging weiter. Auch ein Levit kam zu der Stelle; er sah ihn und ging weiter. Dann kam ein Mann aus Samarien, der auf der Reise war. Als er ihn sah, hatte er Mitleid, ging zu ihm hin, goss Öl und Wein auf seine Wunden und verband sie. Dann hob er ihn auf sein Reittier, brachte ihn zu einer Herberge und sorgte für ihn. Am anderen Morgen holte er zwei Denare hervor, gab sie dem Wirt und sagte: Sorge für ihn, und wenn Du mehr für ihn brauchst, werde ich es Dir bezahlen, wenn ich wiederkomme“ (Lukas 10, 30-35; zit. nach der Einheitsübersetzung der Bibel: Bischöfe Deutschlands et al. 1980). 
auch dasselbe gönnt, was er sich selbst gönnt ${ }^{\star 53}$ ). Theoretisch erscheint daher die Annahme legitim, dass sich religiöse Menschen entsprechend internalisierter religiöser Werte, prosozialer Normen, Glaubensinhalte und deren Kernbotschaften - wie etwa der Nächstenliebe, Brüderlichkeit, der sozialen Mitverantwortung oder Gemeinschaftlichkeit - und aufgrund des Gemeinschaftsbezugs von Religion besonders prosozial, solidarisch, verantwortungsvoll, religionskonform und gottgefällig verhalten dürften. Dies lässt sich auch empirisch nachweisen (u. a. Pickel 2018: 969 f.; Pickel 2011: 43 ff.; Harris 2003). Zentral ist hier die Bedeutung religiöser Sozialisation und Tradierung (Kecskes/Wolf 1996: 89 ff.; Pickel 2011: 409). Aus migrations- und religionssoziologischer Perspektive sind in diesem Zusammenhang - und mit Blick auf den Forschungsgegenstand dieser Arbeit - intergenerationale innerfamiliäre Transmissionsprozesse bedeutsam, die theoretisch eine anhaltend hohe Stabilität von kulturellen Werten und Einstellungen erklären. Dies ist auf eine stärkere Beaufsichtigung der Sozialisation der Kinder und Jugendlichen durch die Eltern sowie auf familiäre Integritätswerte zurückzuführen, die den intergenerationalen familialen Zusammenhalt gewährleisten und damit auch gegen möglicherweise eintretende existenzielle Risiken absichern sollen (u. a. Diehl/Koenig 2009: 303 f.; Nauck 2007; Nauck/Steinbach 2001). Das Engagement von Menschen mit Zuwanderungsgeschichte kann dann in starkem Ausmaß von religiösen Werten angeleitet sein und im Zusammenhang mit der Religionsausübung - ,für die Sache Gottes ' - stattfinden. Des Weiteren kann ein Unterstützungsethos religiös und durch ein theologisch fundiertes Wohlfahrtsverständnis begründet sein, indem die finanzielle und soziale Hilfeleistung für Bedürftige - der ,Dienst am Menschen “ - als eine religiöse Pflicht betrachtet und soziale Probleme und Herausforderungen im Islam als Prüfung Gottes verstanden werden (z. B. Koran, Sure 2:155) (BMFSFJ 2017: 203; Ceylan/Kiefer 2016: $83 \mathrm{ff}$.).

Die Handlungswirksamkeit solcher Normen hängt vom Grad der subjektiven Internalisierung $a b, d . h$. inwiefern sie sich als persönliche Verhaltensstandards zu eigen gemacht wurden. Das Subjekt wird in seinem Handeln von dem Maßstab der Normgerechtigkeit gelenkt und dadurch von einer externen Beurteilung

\footnotetext{
${ }^{53}$ Sahih Muslim, Das Buch des Glaubens, Kapitel 2/Hadithnr. 64 (URL: http://islami sche-datenbank.de/sahih-muslim?chapterno=2\&hadithid=25\&action=display; letzter Abruf am 03.04.2021); s. auch Sahih al-Buchari, Der Glaube, Kapitel 2/Hadithnr. 13: „Anas, Allahs Wohlgefallen auf ihm, berichtete, dass der Prophet, Allahs Segen und Heil auf ihm, sagte: ,Keiner von euch darf sich als gläubig ansehen, bis er seinem Bruder auch dasselbe gönnt, was er sich selbst gönnt “" (URL: http://islamische-datenbank.de/sahih-al-buchari?action=anz eigen\&hadithno=13; letzter Abruf am 03.04.2021).
} 
unabhängiger, da antizipierte negative und positive Folgen einer Selbstbewertung unterzogen werden (Heckhausen 1989: 286). Bei dieser ist das Verhalten - dem normentheoretischen Konzept von Shalom H. Schwartz zufolge - ,motivated by the desire to act in ways consistent with one's values so as to enhance or preserve one's sense of self-worth and avoid selfconcept distress" (Schwartz 1977: 226). Persönlich verbindlich gemachte prosoziale Normen können sich im Rahmen einer Wertungshierarchie gegenüber allgemeinen (sozial vorgegebenen) Normen sogar stärker abheben. Diese Selbstattribuierung bzw. Selbstverbindlichkeit kann psychologisch auch mit dem Begriff der internalen Kontrollüberzeugung gefasst werden: Das Individuum nimmt Ereignisse als Konsequenz des eigenkontrollierten persönlichen Verhaltens wahr (Rotter 1966). ${ }^{54}$ Im Zusammenhang mit dieser Selbstattribution spielen die Normen der sozialen Verantwortlichkeit und der Gegenseitigkeit (Reziprozität) eine Rolle. Ferner ist auch die Bereitschaft und Fähigkeit, sich in andere hineinzuversetzen und deren Perspektive zu übernehmen für prosoziales Verhalten relevant. Eine Rollenübernahme kann eine ,empathische Emotion" bzw. Einfühlung und prosoziales Verhalten auslösen (Heckhausen 1989: 293, 297 f.). Je mehr jemand zu solcher Einfühlung tendiert und fähig ist, desto hilfsbereiter ist er im konkreten Fall (Coke et al. 1978). Aber auch hier ist zu bedenken, dass persönlichkeits- und situationsspezifische Umstände interagieren bzw. miteinander konkurrieren können. ${ }^{55}$ Mit Blick auf die internale Kontrolle vermittels der Internalisierung von Normen, etwa im Falle intrinsischer Religiosität, kann somit auch von einer spezifischen Persönlichkeitsdisposition gesprochen werden, die situationsübergreifend - und nicht nur spontan - in prosozialem Verhalten resultieren kann (Heckhausen 1989: 299).

Neben dem Aspekt der sozialen Fürsorge können empirisch enge positive Zusammenhänge zwischen religiösen Werten und den Wertorientierungen der Traditionalität, Sicherheit, Konformität, Anpassung und Selbstkontrolle und negative Korrelationen mit denjenigen der Selbstentfaltung, Risikobereitschaft und des Hedonismus festgestellt werden (Schwartz/Huismans 1995; Pickel 2018: 969 ff.). Dieses bedeutet jedoch nicht, dass diese konträren Werthaltungen im Rahmen der Sozialisation und eines religiösen Individualisierungsprozesses nicht miteinander gekoppelt und im weiteren Lebensverlauf bedeutsam sein könnten (Pickel 2018: 969 ff.). Zudem wird im Rahmen von Theorien des Wertewandels angenommen, dass religiöse Wertorientierungen durch säkulare ersetzt werden oder sich von

\footnotetext{
${ }^{54}$ Bei einer externalen Kontrollüberzeugung werden Ereignisse hingegen als vom eigenen Verhalten unbeeinflussbar eingestuft.

55 Zumindest vorübergehend unterminieren selbstbezogene Tätigkeiten und Zeitdruck die Bereitschaft und Fähigkeit zur Einfühlung (Heckhausen 1989: 295).
} 
ihrer religiösen Verankerung entkoppeln, sodass z. B. Werte wie Solidarität oder Nächstenliebe in der Bevölkerung unabhängig von konfessionellen Zugehörigkeiten auf eine breite Zustimmung stoßen und quasi „,generalisiert“ werden (Parsons 1977: 279-320; Pickel/Krüggeler 2001; Pickel 2018: 959).

Die Zusammenhänge und Entwicklungen werden jedoch für religiöse, westlich und v. a. christlich geprägte Werte ausgemacht. Für den deutschen Kontext werden in Bezug auf die (auch in dieser Arbeit) im Interessenfokus stehenden Muslime unter Bedingungen kultureller Differenz sehr unterschiedliche Ausprägungs-, Kombinationsformen und Wertorientierungsrichtungen für möglich gehalten (Diehl/Koenig 2009; Nauck 2007; Ceylan 2012a; Nagel 2018a, b). So zeigen jüngere personenbezogene Studien für Muslime eine stärkere Traditionsverhaftung und Sicherheitsorientierung als bei Christen und sowohl bei jüngeren und älteren Altersklassen eine hohe Bedeutung der Werte Hilfsbereitschaft und Hedonismus (Pollack/Müller 2013: 30, 65; Sinnemann 2017: 29, 44). Dies verweist auf eine mögliche Vereinbarkeit von traditionell-konservativen (materialistischen) und individualistisch-hedonistischen bzw. postmaterialistischen Wertvorstellungen.

Für die subjektiven religiösen Persönlichkeitsdispositionen sind familiäre Sozialisation sowie bewusste (Selbst-)Selektion von und durch Organisationen bedeutsam. Gemeinsam vertretene religiöse Grundsätze sowie eine geteilte religiöse Wertebasis motivieren zur Beteiligung in der Organisation. Das Individuum kann entweder in eine religiöse Organisation hineinwachsen oder sich bewusst für ein Engagement in einer solchen entscheiden. Solche wertegeleiteten Verhaltensweisen und ethischen Imperative dürften dann vor anderen Motiven (im-)materieller Natur, eigennützigen Interessen und Vorteilen rangieren. Auch ,säkulare“ Vereine können auf ideologischer oder weltanschaulicher Basis operieren und aufgrund propagierter Werte und Zwecke Mitglieder attrahieren bzw. an sich binden (Clark/Wilson 1961: 146 ff.). Allerdings ist der zentrale Anreiz für ein Engagement in einer religiösen Organisation für eine gläubige Person und deren Lebensführung insofern als stärker einzustufen, als es für das Ausleben und die Bestätigung seines individuellen Glaubens - als identitäts- und lebenssinnkonstitutives Element - eine durch eine religiöse Vereinigung vermittelte, gewährleistete Kommunikabilität benötigt (Pollack 2018: 38 f.; s. I. 1; 4.3.1). Diese betrifft die Kommunikation über den Gottesglauben und religiöse Inhalte und eine gemeinsame religiöse Praxis (wie Rituale, Zeremonien oder Gottesdienste) innerhalb einer Gemeinschaft, in der bestimmte Werte, Normen und Verpflichtungen propagiert und geteilt werden.

Innerhalb gegebener Infrastrukturen religiöser Zusammenschlüsse können sich religiöse Motive emotional Ausdruck verschaffen und entsprechende kognitive Handlungsdimensionen als solche wahrgenommen bzw. realisiert werden (Krech 
1999: 31 f.). Die Befriedigung subjektiver religiöser Bedürfnisse erfolgt im Rahmen von Kommunikabilität und den offerierten Gelegenheitsstrukturen der Gemeinschaft. Die subjektive Glaubensdimension und die Einbindung des Individuums in eine religiöse Gemeinschaft komplementieren und bedingen einander somit gegenseitig. Sie sind insofern zusammenzudenken. Durch Anwesenheit und Agieren der Individuen werden die religiösen Strukturen reproduziert und stabilisiert.

Vor dem Hintergrund der in diesem Kapitel dargelegten theoretischen Prämissen, eines anzunehmenden Primats von religiösen Motiven bzw. Anreizen und der Tragweite von zwischen religiösem Subjekt und Kollektiv wirkenden komplementierenden Effekten wird folgende kontrastierende Doppelhypothese aufgestellt:

$\mathbf{H 3}_{1}$ : Aus Sicht der befragten Funktionsträger rangieren bei den Freiwilligen in religiösen MSO religiöse Wertüberzeugungen (anderen den Glauben nahebringen; religiöse Werte/Gebote umsetzen), gruppenbezogene Solidarität (mit Gleichgesinnten zusammenkommen; Solidarität mit der Organisation) und Hilfeleistung für andere vor anderen nutzenund selbstbezogenen Motiven (Erwerb von Qualifikationen; Erhalt von Gegenleistung).

$\mathbf{H 3}_{2}$ : Die Motive der Freiwilligen, sich in nicht-religiösen MSO zu engagieren, bestehen nach Einschätzung der befragten Funktionsträger mit größerer Wahrscheinlichkeit aus selbstbezogenen Nutzenerwägungen (Erwerb von Qualifikationen; Erhalt von Gegenleistung), aus der Zuteilwerdung von Wertschätzung bzw. Anerkennung sowie aus der genussbetonten Bereicherung des eigenen Lebens (Freude haben).

An dieser Stelle ist nochmal darauf hinzuweisen, dass die Erhebung auf der Mesoebene stattgefunden hat und Funktionsträger der MSO befragt wurden. Die Ergebnisse basieren daher auf deren subjektiven Einschätzungen; es werden also nicht die tatsächlichen Beweggründe der Engagierten auf der Individualebene erhoben. Anzunehmen ist, dass die Funktionsträger persönliche Motive mitgeteilt oder eine „Passung“ von kollektiven Zielen und Interessen der MSO mit den eingeschätzten Motiven der Engagierten vorgenommen haben. Die Motivabfrage besitzt damit quasi eine Dummy-Funktion, eine testende Stellvertreterfunktion, die gleichwohl interessante Auskünfte gibt, da die menschlichen Motive im Kontext organisationaler Rahmenbedingungen betrachtet werden. 


\subsubsection{Das Binnenleben von Organisationen: Dimensionen der formalen Organisationsstruktur}

Die formale Struktur einer Organisation, von der im Folgenden die Rede sein soll, kann als ein System relativ dauerhaft geltender offizieller Vorschriften, Regelungen und Ordnungen verstanden werden, die das Verhalten und die Leistung der Organisationsmitglieder zwecks Erreichung bestimmter Organisationsziele steuern sollen (Kieser/Walgenbach 2010: 15 ff.). Als anschauliches, vereinfachendes Beispiel können die Spielregeln bei einem Fußballspiel dienen, die festlegen, wie viele Personen mitspielen dürfen und welche Handlungen den Spielern oder dem Torwart erlaubt sind (Horch 1983: 2) ${ }^{56}$ Die Regelungen betreffen den Aufbau und die internen Abläufe einer Organisation, Verhältnisse der Unter- und Überordnung sowie die Frage der Honorierung (Entlohnung). Die formale Struktur ist nicht mit dem faktischen Organisationsgeschehen gleichzusetzen, aber sie ist „für die Organisationspraxis der entscheidende Gestaltungs- und Handlungsparameter" (Preisendörfer 2016: 70). Sie wird komplementiert von einer auf emotionale und soziale Bedürfnisse - das soziale Miteinander - der Mitglieder abstellenden informalen bzw. informellen Organisationsstruktur, auf die im vorliegenden Kapitel ebenfalls einzugehen ist.

Aufgrund seiner Prominenz innerhalb der deutschsprachigen Organisationsforschung soll eingangs hinsichtlich ordungsstiftender Regelungen das systemtheoretische Verständnis von formalen Strukturen Erwähnung finden. Luhmann zufolge ist in von der Umwelt abgrenzbaren sozialen Handlungssystemen, ,das faktische Verhalten durch eine Struktur von besonders herausgehobenen formalen Erwartungen geordnet“ (Luhmann 1964: 29). „Formale Organisation [...] besteht aus den Mitgliedsrollen, die das Verhalten definieren, das von einem Mitglied als solchem erwartet wird" (ebd.: 38; 4.3.3). Die formalen Strukturen entsprechen demnach Erwartungen - mitgeteilten Mitgliedschaftsbedingungen -, über die entschieden werden kann. Die entschiedenen Entscheidungsprämissen (Luhmann 2000: 222 ff.) bilden damit nach systemtheoretischer Auffassung die Formalstruktur einer Organisation. Damit ist aus soziologisch-funktionalistischer Perspektive das „Steuerungs-“ oder „Ordnungsproblem“ angesprochen, das die grundsätzliche Frage betrifft, wie aus dem handelnden Zusammenwirken von Individuen

\footnotetext{
${ }^{56}$ Derlei „Spielregeln“ existieren auch in anderen Lebensbereichen. Als ein vorausgesetzter Komplex von Handlungsmustern, an denen Personen ihr Verhalten (un-)bewusst ausrichten, ermöglichen sie gegenseitige Orientierung und Erwartungssicherheit (Türk 1978: 92).
} 
mit unterschiedlichen Motivlagen und Standpunkten, unter Beeinflussung weiterer Umweltbedingungen, eine soziale Ordnung entstehen kann (Luhmann 1964, 2000, 2012/[1987]).

Die in diesem Kapitel im Folgenden zu beleuchtenden Strukturdimensionen können in unterschiedlichem Ausmaß auf eine Vielfalt von Organisationen angewendet werden. Durch die Erläuterung der Strukturbesonderheiten freiwilliger Vereinigungen soll deren Profilierung gegenüber gewinnorientierten Organisationen erfolgen sowie das mit quantitativen empirischen Methoden schwer abzubildende Binnenleben von Freiwilligenorganisationen sowie die darin geltenden „Spielregeln“ veranschaulicht werden.

\section{Fünf zentrale Dimensionen der formalen Organisationsstruktur}

Für grundlegende Orientierung sollen im Folgenden fünf zentrale Bausteine der formalen Organisationsstruktur in einer Komplexität reduzierenden Form dargestellt werden. Konkret handelt es sich um (1) Arbeitsteilung, (2) Koordination, (3) Hierarchie, (4) Delegation und (5) Formalisierung. Sind die drei erstgenannten Gesichtspunkte essenziell, bleiben die beiden letztgenannten in der Fachliteratur eher unberücksichtigt (Preisendörfer 2016: 77). Die Darstellung bezieht sich im Wesentlichen auf die Durchsicht internationaler Literatur, v. a. auf die Explikationen von Alfred Kieser und Peter Walgenbach (Kieser/Walgenbach 2010: 65-190; Preisendörfer 2016: 69-77; s. auch Tab. 4.3).

Die Arbeitsteilung innerhalb einer Organisation bezieht sich auf die Erfüllung von spezifischen Aufgaben, welche die Umsetzung der selbst gesetzten Ziele sowie Effizienzsteigerung gewährleisten sollen. Die Arbeitsteilung ist unmittelbar mit dem Grad der Spezialisierung verbunden, die v. a. durch Ausdifferenzierung bzw. Eingrenzung der Aufgaben, die zu bewältigen sind, gekennzeichnet ist (Kieser/Walgenbach 2010: 81-93; Preisendörfer 2016: 71). Zentrale Fragen lauten, welche Qualifikationen bestimmte Stellen, Aufgabenfelder und Positionen voraussetzen und wie Personen für solche rekrutiert werden können.

Mittels Koordination, dem zweiten Kernelement der formalen Struktur, werden Abstimmung und Harmonisierung von Arbeitsabläufen organisiert. Für Aufteilung, Zuordnung und Regelung aufeinander abzustimmender Arbeitsprozesse identifizieren Kieser und Walgenbach (2010: 102-127) sieben Koordinationsinstrumente (bzw. Koordinationsmechanismen). Die strukturellen Instrumente umfassen Koordination 1.) durch persönliche Weisungen, 2.) durch Selbstabstimmung (Selbstkoordination), 3.) durch Programme und 4.) durch Pläne. Zu den nicht-strukturellen Instrumenten der Koordination gehören 5.) die Standardisierung von Rollen, 6.) die 
organisationsinternen Märkte und 7.) die Organisationskultur. Die sieben genannten Koordinationsinstrumente sollen im Folgenden kurz erläutert werden (s. auch Tab. 4.3).

Koordinations- bzw. Kommunikationsprozesse verlaufen entweder vertikal, d. h. von oben nach unten durch persönliche Weisung (1.) oder eher horizontal, d. h. in einem teamorientierten, basisdemokratischen Stil qua Selbstabstimmung (2.) (Kieser/Walgenbach 2010: 101 ff.). Schriftlich festgelegte Programme (3.) enthalten verbindliche Verfahrensrichtlinien, die den Organisationsmitgliedern dauerhaft vorgegeben werden (ebd.: 107). Ähnliches sollen für einen bestimmten Zeitraum geltende verschriftlichte Pläne (4.) bewirken (ebd.: 111). Der Aspekt der Planungsaffinität wird in der Organisationsforschung auch häufig mit dem Begriff der Standardisierung bezeichnet. Diese bezieht sich auf den Grad der Standardisierung von Rollendefinitionen (5.), die ein Teil langfristiger Personalplanung darstellt und eine sensible Bestimmung von für bestimmte Aufgaben erforderlichen Leistungsbzw. Rollenprofilen sowie Persönlichkeitsmerkmalen notwendig macht.

Der organisationsinterne Markt (6.) soll durch ökonomische Anreizmechanismen die Konkurrenz unter den Mitgliedern innerhalb der Organisation erhöhen und damit die interne Marktsituation beleben. Diesem ökonomischen Konkurrenzmoment steht der Koordinierungsmechanismus der Organisationskultur (7.) gegenüber. Über die Organisationskultur wird versucht, die unterschiedlichen Organisationsmitglieder mit je verschiedener Motiv- und Mentalitätslage miteinander zu verbinden, z. B. über bedeutungsvolle Werte, Glaubensvorstellungen, Rituale, Symbole oder attraktive Unterhaltung, um über die Betonung gewisser Gemeinsamkeiten eine Organisationsidentität (,Corporate Identity“) aufzubauen (Scott/Davis 2007: 23, 219; Tacke 2010: 353; Preisendörfer 2016: 73). Die Vermittlung einer solchen Identität kann für das Selbstverständnis einer Organisation und das im Rahmen der Öffentlichkeitsarbeit nach außen präsentierte Erscheinungsbild zentrale Bedeutung besitzen. Auf das Konzept der Organisationskultur wird im Folgenden noch einmal zurückzukommen sein.

Die dritte Strukturdimension Hierarchie behauptet Geltung v. a. für größere Arbeitsorganisationen wie produzierende Unternehmen, nicht aber für kleine Vereinigungen. Hierarchie (oder: Konfiguration) bedeutet Autoritätsstruktur und betrifft primär die Autorität von Vorgesetzten gegenüber Untergebenen (Pugh et al. 1963: 305). Damit direkt verknüpft ist die Existenz von Verhältnissen der Unter- und Überordnung im Stellengefüge sowie die Regelung von Entscheidungsbefugnissen (Preisendörfer 2016: 74).

Delegation (oder: Kompetenzverteilung) betrifft die Zuweisung von Kompetenzen zu bestimmten Stellen oder Positionen innerhalb der Organisation. Der, Ort “ der 
Autorität innerhalb der Organisationsstruktur wird mit den Begriffen der Zentralisierung bzw. Dezentralisierung erfasst. Entscheidungsbefugnisse werden bestimmten ausgewählten, da qualifizierten Personen übereignet. Zuständigkeiten und Rechte werden dabei offiziell übertragen. Formelle oder institutionelle Autorität kann delegiert werden, nicht delegiert werden kann ,reale oder persönliche Autorität" (Pugh et al. 1963: 304), die auf Wissen, Erfahrungen oder Leistung und dem daraus erwachsenden Einfluss und hohem (fachlichem) Ansehen einer Person beruht. ${ }^{57}$ Die Übertragung von Entscheidungsrechten geht für die Führung einer Organisation immer mit Kontroll- und Machtverlust einher, während sie für weiter unten stehende Organisationmitglieder mit Kompetenzgewinn und Verantwortungszuwachs verbunden ist, die als angenehme Herausforderung und Gratifikation, teilweise aber auch als Belastung empfunden werden kann (Preisendörfer 2016: 75; Preisendörfer 1985).

Formalisierung, die fünfte Strukturdimension, fixiert standardisierte Kommunikation, Abläufe und Strukturen. Die schriftliche Fixierung kann in Form von Organigrammen, (Verfahrens-)Richtlinien, Organisationshandbüchern und Stellenbeschreibungen erfolgen (Kieser/Walgenbach 2010: 157). Die Formalisierung ist ein wesentliches Merkmal der Bürokratisierung und ist nicht mit dem Begriff der formalen Organisationsstruktur bzw. formalen organisatorischen Regeln zu verwechseln, die nicht zwangsläufig formalisiert, $d$. h. schriftlich fixiert, sein müssen.

Die formale Struktur der Organisation wird ergänzt und komplettiert durch die informale Organisationsstruktur, die für intaktes Zusammenwirken der Vorgänge innerhalb einer Organisation und v. a. für die emotionalen und geselligen Bedürfnisse der Mitglieder wichtig ist. Sie übernimmt gegenüber weitgehend präskriptiver Ordnung kompensatorische Funktion (Tacke 2010: 352).

Luhmann zufolge gehören die informalen Strukturen als Teilstruktur zum gleichen sozialen System wie die formalen und bilden „eine andere Verhaltensordnung mit eigenen Normen und Kommunikationswegen, [...] [die] vor allem gefühlsmäßig fundiert und auf die Persönlichkeitsbedürfnisse in der Arbeitssituation zugeschnitten" sind und ,modifizierend in die formale Planung ein[greifen]" (Luhmann 1964: 30). Bei den informalen Strukturen handelt es sich um unentscheidbare, also nicht entschiedene oder explizit formulierte, sondern um implizite und latente Erwartungen (Luhmann 2000: 240). Die informale Struktur wird häufig auch mit dem bereits erwähnten Begriff der Organisationskultur bezeichnet. Gemeint sind ,,spezifische Überzeugungen, Werte und Symbole, die sich in einer Organisation im Laufe

${ }^{57}$ Amitai Etzioni (1975: 154) unterscheidet zwischen formalen Führern, die aufgrund eines Amtes und ihrer Persönlichkeit Macht ausüben können, sowie informellen Führern, die nicht über eine amtliche, sondern nur eine persönliche Autorität verfügen. 
Tab.4.3 Hauptdimensionen der formalen Organisationsstruktur

\begin{tabular}{|c|c|c|}
\hline Dimension & Alternative Bezeichnungen & Kurzcharakterisierung \\
\hline Arbeitsteilung & $\begin{array}{l}\text { - Spezialisierung } \\
\text { - funktionale/horizontale } \\
\text { Differenzierung }\end{array}$ & Aspekte: Ausmaß \& Art der Arbeitsteilung \\
\hline Koordination & - Integration & $\begin{array}{l}\text { In welchem Ausmaß kommen verschiedene } \\
\text { Koordinationsinstrumente zum Einsatz? } \\
\text { Unterschieden werden: } \\
\text { - strukturelle Koordinationsinstrumente } \\
\text { (persönliche Weisung, Selbstabstimmung, } \\
\text { Programme, Pläne) \& } \\
\text { - nicht-strukturelle Koordinationsinstrumente } \\
\text { (Standardisierung von Rollen, organisati- } \\
\text { onsinterne Märkte, Organisationskultur) }\end{array}$ \\
\hline Hierarchie & $\begin{array}{l}\text { - Konfiguration } \\
\text { - vertikale Differenzierung }\end{array}$ & $\begin{array}{l}\text { Aspekte: flache oder steile Rangordnungen; } \\
\text { Leitungsintensität; modernes Projektmanage- } \\
\text { ment etc. }\end{array}$ \\
\hline Delegation & $\begin{array}{l}\text { - Entscheidungsdelegation } \\
\text { - Kompetenzverteilung }\end{array}$ & Ergebnis: Ausmaß der (De-)Zentralisierung \\
\hline Formalisierung & • „Verschriftlichung“ & $\begin{array}{l}\text { Aspekte: Strukturformalisierung (schriftliche } \\
\text { Dokumentation von Organisationsregeln) \& } \\
\text { Informationsflussformalisierung (schriftlich } \\
\text { erfolgende Weisungen, die keine Organisa- } \\
\text { tionsregeln beinhalten) }\end{array}$ \\
\hline
\end{tabular}

Quelle: In Anlehnung an Preisendörfer 2016: 71.

der Zeit entwickelt haben und das Handeln der Organisationsmitglieder informell prägen“" (Schreyögg 2003: 448). Organisationskultur wird im Rahmen organisationsinterner Sozialisationsprozesse vermittelt. Sie beeinflusst Denken, Handeln und Emotionen der einzelnen Organisationsmitglieder i. d. R. implizit, häufig unbewusst und wie selbstverständlich zugleich. Da kollektives, mehr oder minder gleichgerichtetes Denken und Handeln durch Interaktionen der Organisationsmitglieder gemeinsam geschaffen werden, ist Organisationskultur grundsätzlich wandelbar und nicht statisch (ebd.: 450 ff.).

Formale und informale Organisationstruktur werden ergänzt durch die neoinstitutionalistische Perspektive, in der die Existenz einer eigenen Schau- oder Scheinstruktur zur Geltung kommen kann. Um Legitimität zu gewinnen, kopieren Organisationen als erfolgreich und vorbildhaft erscheinende Strukturelemente von Organisationen in ihrer Umwelt, die Bestandteil ihrer eigenen formalen Struktur werden können, die aber auch dazu dienen, nach außen zur Schau gestellt zu 
werden - d. h. als „Legitimitätsfassade“ zu fungieren -, um seitens der gesellschaftlichen Umwelt anerkannt zu werden und als attraktiv, gesetzestreu, innovativ und gesellschaftspolitisch verantwortlich etc. zu gelten (4.5).

\section{Strukturbesonderheiten freiwilliger Vereinigungen}

Vereine, als assoziative Formen formaler Organisationen, verfügen hinsichtlich der Ausgestaltung ihrer Binnenstruktur bzw. inneren Ordnung, die sie sich in Form einer Satzung zu geben haben, über vergleichsweise große Autonomie. Das spiegelt sich zunächst - und dem soll im Folgenden ausführlich Raum gegeben werden - in der juristischen Ordnung, die das BGB dem Verein als Rechtsform gegeben hat. Das BGB fixiert gewissermaßen den Bewegungs(spiel)raum, in dessen Rahmen die meisten MSO agieren.

Der Vereinsvorstand ( $\$ 26$ Abs. 1 BGB) und die Mitgliederversammlung ( 32 Abs. 1 BGB) als oberstes Vereinsorgan sind die gesetzmäßig vorgeschriebenen „Minimalanforderungen“ (Zimmer 2007: 26), durch deren Konstituierung der Verein handlungsfähig wird. Bereits in der Gründungsphase muss sich der Vorstand bilden, um über die Eintragung in das Vereinsregister die Rechtsfähigkeit des eingetragenen Vereins (e. V.) als juristische Person zu erlangen.

Die Satzung soll eindeutig die Zusammensetzung des Vorstandes und die Anzahl der Mitglieder bestimmen ( 58 BGB), wobei die Ämterbezeichnung den jeweiligen Vereinsvorstellungen folgen kann (BMJV 2016: 18). Zumeist werden mit der Bestellung eines Ersten und Zweiten Vorsitzenden, eines Schriftführers und eines Kassenwartes vier Zuständigkeitsbereiche festgelegt (Zimmer 2007: 26). Der Vorstand übernimmt die Rolle des gesetzlichen Vertreters (§ 26 BGB), der die Geschäftsführung wahrzunehmen und den Verein (außer-)gerichtlich zu vertreten hat. Auch über die Modalitäten der Einberufung der Mitgliederversammlung und ihrer Beschlussfassungen kann der Verein grundsätzlich frei entscheiden, sie sollen in der Satzung aber eindeutig bestimmt werden ( $\$ 58$ BGB). Neben der gesetzlich dekretierten Einrichtung einer Mitgliederversammlung und eines Vorstandes kann der Verein weitere frei zu benennende Organe installieren, wie z. B. einen Beirat, einen Ausschuss, ein Kuratorium, einen Verwaltungsrat oder ein Präsidium. Satzungsbestimmungen müssen deren Zusammensetzung, Aufgaben, Verfahrensweisen der Mitgliederbestellung und Verfahrensvorschriften für die Aktivitäten des jeweiligen Organs festlegen, um eindeutige Strukturen und Zuständigkeiten zu etablieren und ,um Meinungsverschiedenheiten zwischen den Organen vorzubeugen“ (BMJV 2016: 19 f.).

Diese flexiblen Gestaltungsoptionen spiegeln die Autonomie des Vereins, der sich selbst in der Satzung festzuhaltende ergänzende Ordnungen (z. B. Ehren- 
oder Beitragsordnungen) geben, aber z. B. auch dem Vorstand eine dominante, starke Stellung einräumen, die Rechte der Mitglieder beschränken oder letztere mit Sonderrechten (z. B. Mehrfachstimmrecht) ausstatten kann (ebd.).

Vor diesem Hintergrund ist die Vielfalt der (migrantischen) Vereine in Deutschland kaum überschaubar. Kleine Vereine - zur Organisierung von Geselligkeit, Unterhaltung und lokaler Kultur - existieren neben großen traditionsreichen Sportund professionell von Hauptamtlichen geführten Dienstleistungsvereinen oder fragilen, ausschließlich auf der Initiative von Engagierten basierenden Selbsthilfevereinen (Zimmer 2011: 461 f.). Auch große (Interessen-)Verbände sind häufig in der Rechtsform des eingetragenen Vereins organisiert. Thomas Lemmen (2002: 29-33) hat für die islamischen Verbände auf die Existenz eines zentralistischen, dezentralistischen, föderativen und Mischformenmodells und damit auf das vorhandene Spektrum ihrer Organisationstrukturen hingewiesen. ${ }^{58}$

Heinz-Dieter Horch $(1983,1985)$ konstatiert, dass auch in freiwilligen Vereinigungen Elemente formaler Organisationsstrukturen zu finden seien, unterstreicht aber den großen Handlungs- und Improvisationsspielraum, über den freiwillige Vereine jenseits der verschriftlichten Satzungsbestimmungen im Gegensatz zu (Industrie-)Betrieben und Verwaltungen verfügen (Horch 1983: 2). In Anlehnung an systemtheoretische Ansätze und Überlegungen zu Kleingruppen stellt Horch

\footnotetext{
${ }^{58}$ Lemmen identifiziert zentralistische Organisationsstrukturen für den Verband der Islamischen Kulturzentren e. V. (VIKZ) sowie die Islamische Gemeinschaft in Deutschland e. V. (IGD). Hier ist lediglich der Verband an seinem Hauptsitz als Verein organisiert und besitzt die Rechtsfähigkeit, während die Ortvereine als Zweigstellen fungieren. Die Beziehungen zwischen den lokalen Niederlassungen und dem Verband sind in der Satzung geregelt. Die Zuständigkeit für alle rechtlichen Vorgänge liegt damit beim Verband.

Um eine dezentralistisch strukturierte Organisation handelt es sich bei der TürkischIslamischen Union der Anstalt für Religion e. V. (DİTIB). Sowohl der Verband als auch die einzelnen Ortsvereine sind eigenständige Vereine, wobei sich die lokalen Vereinigungen dem Verband angeschlossen haben, ihre Angelegenheiten selbstständig rechtlich regeln und ihre Vorstände in der Mitgliederversammlung bestellen. Die Vereinssatzung enthält Bestimmungen über das Verhältnis zwischen Verband und Mitgliederorganisationen.

Föderativ strukturierte Organisationen, wie die Föderation der Türkisch-Demokratischen Idealistenvereine in Europa e. V. (ADÜTDF), die Union der Türkisch-Islamischen Kulturvereine in Europa e. V. (ATIB) und die Föderation der Alevitischen Gemeinde Deutschland e. V. $(\mathrm{AABF})$, werden erst durch den Zusammenschluss von eigenständigen Vereinen begründet. Letztere müssen ihre Selbstständigkeit nicht aufgeben, sich allerdings an den Geschäften der Föderation beteiligen, die daher über große Vorstände und zusätzliche Organe wie Kontrolloder Aufsichtsräte verfügt. Mischformen vereinen in ihrem Aufbau (de-)zentralistische und föderative Strukturelemente; ein Beispiel hierfür bietet die Islamische Gemeinschaft Millî Görüş (IGMG) (Lemmen 2002: 29-33).
} 
die Eigenarten der Struktur freiwilliger Vereinigungen ,in einem bipolar konstruierten Idealtypus“ (ebd.: 6) den erläuterten strukturellen Charakteristika größerer Organisationen gegenüber (Tab. 4.4).

Tab. 4.4 Strukturbesonderheiten von Betrieben/Verwaltungen versus Freiwilligenorganisationen

\begin{tabular}{ll}
\hline $\begin{array}{l}\text { Besondere Eigenschaften von } \\
\text { Betrieben/Verwaltungen }\end{array}$ & $\begin{array}{l}\text { Besondere Eigenschaften von } \\
\text { freiwilligen Vereinigungen }\end{array}$ \\
\hline Konsumentenhaltung/Kommerzialisierung & Unwirtschaftliche, kulturelle Interessen \\
\hline Bezahlte Mitarbeit & Unbezahlte, freiwillige Mitarbeit \\
\hline Ökonomische Anreize für die Mitgliedschaft & $\begin{array}{l}\text { Gemeinsame Zielorientierung; } \\
\text { Gruppenbindung }\end{array}$ \\
\hline Zugang zu Ressourcen von Nicht-Mitgliedern & Abhängigkeit von Mitgliederressourcen \\
\hline $\begin{array}{l}\text { Oligarchie } \\
\text { (= eine kleine Gruppe übt die Herrschaft aus) }\end{array}$ & Demokratische Entscheidungsstruktur \\
\hline Bürokratie (vorschriftenmäßig) & Gruppencharakter \\
\hline
\end{tabular}

Quelle: Eigene Zusammenstellung als Modifikation von Horch 1983: 6.

Horch (1983: 33-98, 109-129) konzentriert sich bei seiner detaillierten Analyse der Strukturen freiwilliger Vereinigungen auf verschiedene Aspekte sowie allgemeine und spezifische Regeln, die sich wie folgt benennen lassen:

1) die Arbeitsteilung und die aufgabenbezogene Verhaltenssteuerung, die durch Personalisierung und Ambivalenz von Verhaltenserwartungen gekennzeichnet ist; sie kontrastiert mit einer in großen Organisationen vorzufindenden Spezialisierung im Aufbau (Ausdifferenzierung von Stellen und Positionen) und der Standardisierung (von spezifischen Rollendefinitionen und Abläufen);

2) die Frage nach der Einbindung unterschiedlicher Mitgliedertypen und den Mechanismen zur Herstellung von Ordnung angesichts geringer Mitgliedschaftsanforderungen;

3) Dimensionen sozialer Kontrolle (Sanktionen);

4) Koordination der Arbeitsteilung;

5) die demokratische Entscheidungsstruktur als für freiwillige Vereinigungen charakteristisches Element anstelle eines hierarchischen Aufbaus. 
Im Folgenden soll zunächst auf die spezifischen Regeln der Arbeitsteilung und die aufgabenbezogene Verhaltenssteuerung (1.) eingegangen werden. Die forschungsleitenden Fragen dieser Arbeit, welche sich u. a. auf das Aktivitätsspektrum von migrantischen Vereinigungen beziehen, implizieren die Frage nach der Arbeitsteilung zwischen Hauptamtlichen und Freiwilligen. Es ist davon auszugehen, dass sich diese in den personell sehr disparat ausgestatteten MSO sehr unterschiedlich ausgestaltet. Bei ressourcenstarken, professionell arbeitenden MSO können vergütete Stellen besetzt werden. Dies kann eine Aufgabendelegation an Freiwillige erforderlich machen und ggf. in Konkurrenzsituationen und Konflikten zwischen den Hauptamtlichen und den Engagierten resultieren (u. a. Borggrefe et al. 2012: 316, Fn 6; Friedrichs et al. 2020: 47). Der Umfang der Aufgabenübernahme durch Freiwillige sowie die Art der Tätigkeitsverteilung bei Verfügbarkeit von Hauptamtlichen sind wiederum Indizien für die Leistungsfähigkeit sowie den Spezialisierungsgrad der MSO, auf den im Folgenden noch näher einzugehen ist.

Horch (1983: 35) unterscheidet zwischen drei Mitgliedertypen: den unbezahlten und bezahlten Mitarbeitern sowie Nicht-Mitarbeitern ${ }^{59}$ (4.3.3). Er sieht die Arbeitsteilung auf einem geringen Niveau angesiedelt (ebd.: 96). Jenseits der mit bestimmten Aufgaben verknüpften Ämter und Positionen seien weitere Pflichten nur vage festgelegt und der Formalisierungsgrad der Anforderungen an die Mitglieder sei generell gering. Dies sei ein ,Charakteristikum der meisten freiwilligen Vereinigungen“ (ebd.: 33) und auf den Aspekt der Freiwilligkeit der Tätigkeitsübernahme zurückzuführen.

Der Grad der Spezialisierung - und Professionalisierung - in freiwilligen Vereinigungen kann anhand der Anzahl ehrenamtlicher Positionen und in stärker ausdifferenzierten Vereinigungen mit einem neben- bzw. hauptamtlichen Bereich anhand der Anzahl bezahlter Mitarbeiter gemessen werden. Dabei ist die freiwillige Übernahme eines Amtes mit bestimmten Erwartungen und Verpflichtungen verbunden. So müssen Vorstandsmitglieder den Verein vertreten und seine Geschäfte führen, die Beschlüsse der Mitgliederversammlung umsetzen, letzterer Auskunft geben und für ihr Tun Rechenschaft ablegen. Vorstandmitglieder übernehmen damit eine verantwortungsvolle Mitarbeiterrolle (ebd.: 63).

\footnotetext{
${ }^{59}$ Die von Horch gewählte Mitgliedertypologie basiert auf fünf Kriterien: 1.) (Nicht-) Anwesenheit; 2.) (Nicht-)Teilnahme an der Vereinsarbeit und an Entscheidungsprozessen; 3.) (Nicht-) Übernahme von einzelnen Aufgaben und (un-)bezahlten Ämtern; 4.) Art des Outputs der Organisation für das Mitglied, d. h. Art der Anreize, die zur Mitwirkung führen; 5.) Input als Art des Beitrags bzw. der spezifischen Aufgaben durch das Individuum für die Vereinigung (z. B. Vorstandsarbeit) (Horch 1983: 35 f.).
} 
Im Rahmen seiner theoretisch und empirisch substantiierten Arbeit ${ }^{60}$ kommt Horch zu dem Schluss, dass in Vereinen nur wenige Positionen einen (wie oben beschriebenen) Formalisierungsgrad aufweisen. Dies sei etwa bei der Rolle des Schriftführers (ebd.: 82) der Fall oder bei der geistlichen Leitung. Diese Ämter werden häufig von Personen übernommen, die die Tätigkeiten aufgrund ihres (religiösen) Wissens, ihrer Ausbildung oder beruflicher Qualifikationen adäquat ausführen können (Lemmen 2002: 28; Halm/Sauer 2015: 59). Generell ist davon auszugehen, dass bei der Rekrutierung von freiwillig und unbezahlt tätigen Mitgliedern eher keine spezifischen, sondern eher allgemeine Kenntnisse und Fähigkeiten vorausgesetzt werden können (Horch 1983: 84). Die Mobilisierung von Mitgliedern und unbezahlten Mitarbeitern erfolgt in freiwilligen Vereinigungen häufig über persönliche Kontakte und Gespräche, Selektion und Selbstselektion (4.3.3.1; 4.4).

Bei vorhandenen Bewerbern für ein Amt sind deren Bekanntheitsgrad, berufliche Kompetenz und die Bewährung in anderen Aufgabenbereichen bedeutsam. Relevant ist insbesondere die Selbstselektion, die sich darüber vollzieht, dass bestimmte verantwortungsvolle Aufgaben Persönlichkeiten anziehen, die sich dafür bestimmt sehen (Horch 1983: 100, 108). Die Anzahl von zu rekrutierenden bzw. rekrutierbaren Mitarbeitern und Engagierten ist wiederum abhängig von den Ressourcen, der Größe und dem Aufbau einer Vereinigung (s. auch Halm et al. 2012a: 7, 78, 86 f., 117; Halm/Sauer 2015: 31).

Geringe Spezialisierung bewirkt nach Horch (1983: 85) eine Personalisierung und „hohe Verfügbarkeit der Rolle“, die „Handlungs- und Interpretationsspielräume“ eröffne und mit individuellen Leistungen ausgefüllt werden könne. Dadurch würden die Identifikation mit der Tätigkeit und die Entwicklung einer ,Ich-Identität“ gefördert, die Tätigkeiten aber auch von den individuellen Eigenschaften, Fertigkeiten und Leistungen konkreter Personen geformt, die damit nicht so einfach auszuwechseln seien.

Niklas Luhmann (1964: 151) konstatiert mit Blick auf solche Möglichkeiten eine für das System der freien Vereinsstruktur funktionale „Entformalisierung oder Entbürokratisierung", die eine Unsicherheit der Mitglieder ob ihrer Pflichten bewirke, was sie ,,aufnahmebereit für neuartiges Verhalten“" mache. „Die Grenzen der ablehnbaren Zumutungen werden ausgedehnt und verschwimmen“ (ebd.). Hans Geser (1980: 227) stellt in diesem Zusammenhang eine ,,Polyvalenz“ als Substitut für strukturelle Differenzierung“ heraus. Die personelle Polyvalenz erfolge „durch Rekrutierung oder Sozialisierung von Individuen, die dank vielseitiger Qualifikation und sachunspezifischer Teilnahmemotivation für die Bewältigung eines breiten

${ }^{60}$ Durchführung von Intensivinterviews und teilnehmenden Beobachtungen. 
Spektrums verschiedenartiger und unvorhersehbar variierender Aufgaben verfügbar sind.“ Die soziale Polyvalenz entstehe „durch Kultivierung besonders flexibler, unabhängig von spezifischen Zwecksetzungen oder Rollendefinitionen stabilisierter Interaktionsbeziehungen und Strukturformen" (ebd.).

Die möglichst längerfristige Einbindung unterschiedlicher Mitglieder und Mitarbeiter (2.) in die Organisation betrifft die Herstellung einer möglichst dauerhaften Verbundenheit zwischen Einzelpersonen mit dem sozialen Handlungssystem und deren verlässliche Verhaltensstabilisierung. Je nach ihrem Charakter kann eine Vereinigung unterschiedliche Anreize bieten, die für die von Horch differenzierten drei Mitgliedertypen - unbezahlte und bezahlte Mitarbeiter sowie Nicht-Mitarbeiter - in Abhängigkeit vom Grad ihrer Involvierung wiederum unterschiedlich bedeutsam sein können (s. auch 4.4). Aktive Mitarbeiter, die ein bestimmtes Amt innehaben und im Gegensatz zu Nicht-Mitarbeitern an spezifische Verpflichtungen und Regeln gebunden sind, dürften auch aufgrund ihrer häufigeren Präsenz die Ausgestaltung der Organisationsstruktur beeinflussen. Im Rahmen der vorliegenden Arbeit ist insbesondere die Einbindung von freiwillig Engagierten, die für ihre Tätigkeiten nicht bezahlt oder die eine Aufwandsentschädigung erhalten, von Interesse. Wie bereits in dem Kapitel zu der Ressourcenausstattung (4.3.2) von nicht gewinnorientierten Organisationen dargelegt, stellen die Freiwilligen für letztere die konstitutive und bestandserhaltene Komponente dar. In diesem Zusammenhang konstatiert Horch: „Je wichtiger die Ressourcen eines bestimmten Mitgliedertyps für die Zielerreichung der Vereinigung sind, um so stärkeren Einfluß hat die Lösung des Einbindungsproblems dieses Mitgliedertyps auf die Struktur der Vereinigung“ (Horch 1983: 37 f.). Somit ist davon auszugehen, dass gerade die Freiwilligen den Strukturdimensionen, wie der Arbeitsteilung und Koordination, ein besonderes Gepräge geben.

Je mehr Anforderungen an eine Mitgliedschafts- oder Mitarbeiterrolle gestellt werden, desto größer fällt die Verhaltenssteuerung ins Gewicht und desto formalisierter ist die Organisation (Mayntz 1963: 86; Luhmann 1964: 31 f.). Wie oben dargelegt zeigt sich der Grad der Formalisierung anhand von Konkretisierung, Detailliertheit und der schriftlichen Fixierung der Regeln wie auch der Leistungskontrolle (Sanktionierung). Renate Mayntz führt für den Organisationstypus Verein treffend aus:

„Die Organisationsleitung hat nur eine sehr begrenzte Verfügungsmacht über die Mitglieder, ausgenommen über die festangestellten Funktionäre oder Experten. Die einfachen Mitglieder wirken freiwillig mit und können schon deshalb nur zu Wenigem fest verpflichtet werden. [...] Da nicht vorherzusagen ist, wie viele Mitglieder wie lange und wie eifrig mitarbeiten werden, läßt sich auch schlecht ein für allemal regeln, 
wer was wann tun soll. Improvisation und Disposition spielen deshalb eine große Rolle beim Handeln in demokratisch aufgebauten Vereinigungen“ (Mayntz 1963: 87 f.).

Wenn in freiwilligen Vereinigungen keine offiziellen Regeln bestehen, müssen Verhaltenserwartungen und Verhaltensweisen über andere Wege eingelöst und gewährleistet werden. Dies geschieht über „Interaktionsbindungen“ (Luhmann 1977: 301), die Einstellungen und Überzeugungen der Handlungspartner offenlegen und ihr Verhalten kalkulier- und voraussehbar machen (vgl. auch Luhmann 1964, 2005d/[1975d]: 67 ff., 2012/[1987]: 414). Regelmäßige Interaktionen bedingen, dass die Handlungspartner Verständnis und Gespür füreinander entwickeln und sich im gemeinsam erfahrenen Lebenskontext der freiwilligen Vereinigung über gemeinsam praktizierte Tätigkeiten konsensuell verständigen, eventuell auch angleichen können (Horch 1983: 67). Die Entwicklung von kongruenten Einstellungen und Erwartungen ist also nicht nur auf ein gemeinsam verfolgtes Organisationsziel, sondern auf diese Interaktionsmuster zurückzuführen. Diese können auch emotional - etwa durch einvernehmlich befürwortete Werte, Rituale und kulturelle Präferenzen - stabilisiert werden (Luhmann 1964: 80, 376; Blau 1964: 280). Hier wird deutlich, dass derlei gefühlsmäßiges Eingebundensein das individuelle Verhalten lenkt und zu tätiger Mitwirkung in der Vereinigung animiert (vgl. auch 4.4.1).

Da die Handlungserwartungen gegenüber den Mitgliedern in freiwilligen Vereinigungen eher gering ausfallen, ist zu erwarten, dass Mechanismen zur Kontrolle bzw. Sanktionierung (3.) abweichenden Verhaltens in der Gruppe auf einem niedrigen Niveau angesiedelt sind. Intrinsische Beweggründe sorgen dafür, sich in irgendeiner Weise in die Vereinigung einzubringen: „Allgemein kann man sagen: je fähiger und bereitwilliger die Mitglieder sind, die ihnen zugeteilten Rollen zu spielen, um so [sic] weniger sind eine streng hierarchische, mit empfindlichen Strafsanktionen ausgestattete Befehlsgewalt und eine genaue Überwachung aller ausführenden Tätigkeiten notwendig“ (Mayntz 1963: 99).

Formalisierte Sanktionsmaßnahmen können durch in der Satzung enthaltene Bestimmungen für den Ausschluss aus der Vereinigung (oder dessen Androhung) sein, wenn z. B. eine gravierende Verletzung der Mitgliederpflichten vorliegt (BMJV 2016: 49). Ebenso können Amtsträger einfach nicht wiedergewählt werden. Ansonsten sind im Gegensatz zu großen Organisationen, die einen hohen Grad der Spezialisierung und Formalisierung aufweisen, keine spezifischen Instanzen für Sanktionen vorhanden. Die Beeinflussung und Steuerung von Verhaltensweisen und Einstellungen der Organisationmitglieder erfolgt vielmehr durch die Kontrolle in der Gruppe, die Nicht-Konformität nicht billigt. Die Kohäsion innerhalb einer Gruppe und die Aufrechterhaltung der inneren Organisationsordnung können durch die Verdeutlichung einzuhaltender Regeln und Normen erfolgen, z. B. über positive 
Aspekte wie Lob, Anerkennung und Belohnung, aber auch über negativ konnotierte Instrumente wie Zurechtweisung, Tadel und Kritik (Horch 1983: 91 ff.).

Eine Überwachung des Mitgliederverhaltens, so sie erforderlich sein sollte, erfolgt durch die Gesinnung der Gruppe: Abweichler können verbal im Rahmen von (Vieraugen-)Gesprächen auf ihr Fehlverhalten aufmerksam gemacht werden ${ }^{61}$. Aber auch mit nonverbalen Mitteln, etwa durch ein demonstratives Schweigen, ein latentes Abkühlen der Atmosphäre oder durch Isolierung des Individuums von der Gruppe kann nicht gebilligtes Verhalten geahndet werden (ebd.: 94 f.).

Luhmann zufolge findet man insbesondere in undifferenzierten sozialen Handlungssystemen eine gefühlsgesteuerte, vertrauensbasierte wechselseitige Befriedigung sowie als Informations- und Gefühlsträger fungierende Kommunikationen, die ein geplantes System von Sanktionen überflüssig erscheinen lassen (Luhmann 1964: 90 f.). Als ein weiteres bedeutsames sublimes, manipulatives Kontrollmittel thematisiert Luhmann das der „Moralisierung“, mit dem der Einzelperson deutlich gemacht werden soll, was wechselseitig geachtet und akzeptiert werden kann, um „die Verhaltensmöglichkeiten der Beteiligten auf ein für das System erträgliches Maß an Varietät“ (Luhmann (2005b/[1975b]: 38 f.) zu begrenzen.

Koordination (4.) gestaltet sich aufgrund beschriebener Charakteristika anders als in stark arbeitsteilig organisierten Unternehmen. Wie oben dargelegt werden Verhaltenserwartungen insbesondere innerhalb sozialer Interaktion festgelegt. Zentralisierung von Entscheidungsbefugnissen, persönliche Weisungen wie auch die detaillierte Planung von Aktivitäten können in freiwilligen Vereinigungen zum einen durch intrinsische Koordinationsmechanismen ersetzt werden, da das Handeln der Mitglieder über die Internalisierung von Werten und Zielen gelenkt und konkrete Entscheidungen zudem einzelnen Mitgliedern anheimgestellt werden (Horch 1983: 96 ff.). Persönliche Anordnungen von Führungsfiguren spielen aufgrund der auf Freiwilligkeit beruhenden und zu stimulierenden Mitwirkung eine untergeordnete Rolle: „Wollen die Leiter [...] bestimmte Aktion(en) durchführen, bei der sie die aktive Mitwirkung aller Mitglieder brauchen, dann können sie selten anordnen, sondern müssen bitten, mahnen und überzeugen“ (Mayntz 1963: 88).

\footnotetext{
${ }^{61}$ Horch (1983: 93 f.) führt ein Beispiel aus einem Intensivinterview an, in dem seitens der interviewten Person als persönliche Hilfestellung gemeinte Kritik an einem innerhalb seiner religiösen Vereinigung aufgefallenen Fehlverhalten erwähnt wurde: „Meinen Sie, wenn ein Ältester [...] etwas tut, was gemäß der Bibel nicht richtig ist? [...] Ja, dann bekommt er das Dienstamt natürlich weggenommen. Der ist dann nicht mehr befähigt, der Versammlung in rechter Weise vorzustehen. Das ist ja klar. Das wurde z. Zt. der Apostel auch getan, und das ist natürlich anhand der Bibel zu rechtfertigen. Man versucht aber, in guten Worten anhand der Bibel ihm klarzulegen, daß er Fehler begangen hat, und man gibt ihm Gelegenheit, eben wieder auf den Weg des Lebens zu kommen [...].“
} 
Extrinsische Koordinationsmechanismen betreffen in freiwilligen Vereinigungen zum anderen die motivierende und koordinierende Funktion einer mit persönlicher oder fachlicher Autorität ausgestatteten Führungsperson, zugleich aber auch den Mechanismus der Selbstabstimmung. Diese kann in Komitees und Ausschüssen erfolgen, ist aber zumeist weniger konkret festgelegt und findet z. B. bei Versammlungen, informell und ad hoc statt. Hier kann es durchaus chaotisch zugehen und es besteht ein höherer Unsicherheitsfaktor, inwieweit Aufgabenzuweisungen in der Praxis tatsächlich realisiert werden (können). Ein charakteristisches Element für freiwillige Vereinigungen ist die Feedback-Koordination oder das „Durchwursteln“ (Horch 1983: 97 f.). Dabei geht es um eine behelfsmäßige, kurzfristige Bereinigung von Fehlern sowie Veränderung ungünstiger Verhaltensweisen von verantwortbaren Personen, wobei über diesbezügliche Erfolge in der Praxis an dieser Stelle nur spekuliert werden kann.

Die demokratische Entscheidungsstruktur (5.) wird häufig als ein wesentliches Charakteristikum von freiwilligen Vereinigungen unterstellt (Zimmer 2007: 35, 66; Zimmer 2011: 454; Tocqueville 1985/[1840]: 248). Auch die rechtlichen Vorgaben des BGB für den eingetragenen Verein, wie etwa das konstituierende Element der Mitgliederversammlung mit ihrer hervorgehobenen Rechtsstellung, implizieren basisdemokratische Eigenschaften. Wenn sich die „,von unten“ durch die Mitglieder konstituierten Vereine durch eine „Bottom-up-Hierarchie“ auszeichnen, bedeutet dies jedoch nicht zwangsläufig, dass Entscheidungen basisdemokratisch getroffen werden; jedoch sollten die Entscheidungen grundsätzlich der Kontrolle der Mitglieder unterworfen sein und ihnen gegenüber verantwortet werden (Borggrefe et al. 2012:314). Einschränkend zu vermerken und bereits thematisiert worden ist, dass sich der Verein durch Bestimmungen in der Satzung allerdings auch eine eigene innere Ordnung geben und den einzelnen Organen unterschiedliche Kompetenzen und besondere Rechte zugestehen kann. Demnach ist nicht bei allen auf freiwilliger Mitgliedschaft und Mitarbeit basierenden Vereinigungen von einer demokratischen Struktur auszugehen, die allen Mitgliedern für ihre Beteiligung und Interessendurchsetzung gleiche Optionen einräumt (Horch 1983: 109, 128).

Das Element der demokratischen Entscheidungsstruktur ist für die Mitglieder und Mitwirkenden wiederum ein Vehikel, über das sie sicherstellen können, dass ihre Interessen in der und durch die Vereinigung vertreten und dass sie auch hinsichtlich einer gemeinsamen Zweckfestlegung adäquat eingebunden werden (Schmitter/Streeck 1999/[1981]: 19; Streeck 2017/[1987]: 528 ff.; Horch 1983: 110, 128). Neben dieser steuernden Funktion ist die demokratische Entscheidungsstruktur aber auch für den Informationsfluss und die Konsensbildung in der Vereinigung bedeutsam. Aus den Beteiligungsmöglichkeiten, dem Gemeinschaftscharakter und 
aus individueller Leistungserbringung generieren sich positive, im besten Fall sich wechselseitig verstärkende Effekte.

Neben relativ demokratischen Entscheidungsstrukturen können Vereinigungen zugleich auch Tendenzen zur „Oligarchie der Aktiven“ (Horch 1983: 128) aufweisen. Demnach kann einer kleinen Gruppe von stark engagierten Mitgliedern, die viel Verantwortung übernehmen und Zeit in die Vereinsarbeit investieren, besonderer Einfluss mit größeren Handlungsspielräumen zukommen. Dabei kann ihr bestimmendes Gewicht jedoch von anderen Mitgliedern durchaus geschätzt und geradezu eingefordert werden: „Ein wichtiger Teil der Arbeit, die man von ihnen erwartet, ist gerade, daß sie Entscheidungen fällen" (ebd.).

\subsubsection{Ebenen und Dimensionen der Organisationsumwelt}

Jede Organisation ist in eine spezifische Umwelt eingebettet, die ihr fortlaufend Impulse gibt und an die sie sich anpassen muss. Die Umwelt umfasst alle wichtigen Elemente außerhalb einer Organisation, ihre äußeren, d. h. in Geschichte und Gegenwart zu verortenden Existenzbedingungen sowie die günstigen bzw. ungünstigen Voraussetzungen, unter denen die Ziele und Zwecke der Organisation realisiert werden können.

Organisationsumgebungen sind dynamisch. Organisationen sind insofern autark, als sie im Inneren Kommunikationen entwickeln, die Eigenes, von der Umwelt Abgrenzbares gründen wie befördern. Hinsichtlich ihrer Existenzberechtigung und Überlebensfähigkeit sind sie jedoch zugleich auf Umweltbedingungen vielfältiger Art bezogen und ausgerichtet (Scott/Davis 2007: 19), und zwar in dem Maße, wie Wille und Notwendigkeit zur Auseinandersetzung mit anderen Akteuren und anderen gesellschaftlichen Systemen gegeben sind. Das betrifft Organisationen als rationale, natürlich-soziale und zuvorderst offene Systeme, die am stärksten, ,in einem vielfältigen Austausch- und Abhängigkeitsverhältnis zu ihrer Umwelt" stehen (Preisendörfer 2016: 150; vgl. für religiöse Organisationen Beckford 1975: 29 ff.), wobei zumeist Strukturen und Vorgänge der Umgebung, sofern sie auf die Organisation einwirken, im Mittelpunkt von Analysen stehen anstatt umgekehrt. Organisationstheorien, die Anspruch auf wissenschaftliche Reliabilität haben, kommen daher gar nicht umhin, die je spezifischen Wechselwirkungen zwischen Organisation und Umwelt begrifflich auszudrücken, denn andernfalls sind die Tätigkeiten der Organisationen, ihre Erfolge oder Misserfolge, die Wahl der organisationalen Strukturen etc. nicht zu erklären (Preisendörfer 2016: 150 f.; Scott/Davis 2007: 106). 
Was hier als „Umwelt“ bezeichnet wird, bezieht sich auf alle relevanten rechtlichen, sozialen, kulturellen, politischen und wirtschaftlichen Rahmenbedingungen, ggf. auch auf Austauschbeziehungen im inter- bzw. transnationalen Kontext. Wie bereits thematisiert wurde, agieren MSO in einem Spannungsverhältnis von Erwartungen und Ansprüchen der Eigengruppe einerseits sowie spezifischen Akteurkonstellationen $^{62}$ der Aufnahmegesellschaft andererseits (Pries 2010a: 39; Schimank 2000: 310 f.). Somit bestehen Wechselwirkungen zwischen Binnenleben und Umwelt, wobei beide nicht ,blind ' interagieren: Die Wahrnehmung und Bewertung gesellschaftlicher Vorgänge und wechselseitiger Behandlung stehen im Mittelpunkt und beeinflussen Handeln, Umgangsformen und Auftreten der MSO (Pries 2013a: 5; Pries 2010a: 20; Nagel 2015b: 31).

Um die Einflussnahme relevanter Umweltbedingungen einzuhegen, wird zunehmend auf den Begriff des organisationalen Feldes rekurriert (Scott/Davis 2007: 269 ff.). Das organisationale Feld stellt einen spezifischen Ausschnitt der externen Bedingungen dar, das die durch die MSO als Kollektivakteure ,wahrgenommenen Handlungsgelegenheiten, Anspruchsgruppen und Legitimationserfordernisse“ (Pries 2010a: 50) absteckt und das „die Gesamtheit aller (anderen) Organisationen bezeichnet, die für eine bestimmte Organisation als Bezugseinheiten und Legitimationsadressaten ihres kollektiven Handelns bedeutsam sind“ (ebd.: 24; s. auch 4.5).

Der gesellschaftliche Kontext, in dem die Organisationen agieren, kann seitens der Organisationen bzw. ihrer Repräsentanten sehr unterschiedlich wahrgenommen werden: als Quelle von Ressourcen, Opportunitäten und Chancen einerseits sowie unter der Perspektive von Anforderungen, Zwängen, Konkurrenz, Bedrohungen und Restriktionen andererseits (Scott/Davis 2007: 19 f.; Preisendörfer 2016: 150 f.). In den Blick geraten in diesem Zusammenhang situative Gegebenheiten und Rahmenbedingungen in Form gesetzgeberischer oder spezifischer finanzieller oder ideeller Fördermaßnahmen, die Verleihung von Privilegien durch die Einräumung von Steuerbegünstigungen ${ }^{63}$ oder das Eingehen von Kooperationen mit anderen Akteuren. Derlei Kontextbedingungen stellen Anreiz- und Gelegenheitsstrukturen dar, die die Ressourcenausstattung, die Handlungsfähigkeit und Performanzen der Organisationen wesentlich mitbestimmen können (s.

\footnotetext{
${ }^{62}$ Akteurkonstellationen betreffen Interessenpositionen, Konflikt- und Kooperationsbeziehungen.

${ }^{63}$ In diesem Zusammenhang spielt der parity claim, der Regulierungsanspruch der Muslime, ebenfalls den Körperschaftsstatus erlangen zu können, in der deutschen Religionspolitik eine dominante Rolle (3.2). Mit dem Körperschaftsstatus werden besondere Rechte verliehen, wie z. B. das Recht auf Steuereinzug unter Mitgliedern oder die Dienstherrenfähigkeit, die es ermöglicht, die Rechtsstellung von Personal auszugestalten.
} 
dazu auch 3.2). Die Rahmenbedingungen sind aktiv gestaltbar bzw. regulierbar: durch unterschiedliche Politikfelder bzw. staatliche Stellen, durch andere Organisationen, institutionelle Arrangements oder den sozialen Kontext; auf vielfältige Art können sowohl Möglichkeiten geboten als auch Zwänge und Druck erzeugt sowie (politische) Kontrolle ausgeübt werden (Scott/Davis 2007: 20; Pugh et al. 1963: 312). In diesem Zusammenhang wird aus politikwissenschaftlicher Perspektive die Bedeutung von Regeln und Regulierungen in staatlicher Gestaltung oder im Kontext von Governance-Systemen betont (3.2; u. a. Benz et al. 2007a; Mayntz 2004; Schuppert 2011).

In Bezug auf die Organisationsforschung ist an dieser Stelle noch einmal herauszustreichen, dass die Orientierungen und Interessen der Mitglieder als die soziale Basis der Organisation einerseits sowie die Erwartungen und Anpassungsforderungen der institutionellen Umwelt andererseits in einem Wechselverhältnis zueinander stehende Interaktionslogiken darstellen. Diese Mechanismen unterliegen der Sozialintegration nach innen (,Mitgliedschaftslogik“) und der Systemintegration nach außen („Einfusslogik“) (Streeck 2017/[1987]: 528; Lockwood 1979: 125). Diese beiden Logiken können miteinander konfligieren, Friktionen verursachen und damit einen Interessenausgleich in Form von Kompromissen oder der Anwendung von spezifischen Strategien notwendig machen.

Folglich besteht ein Spannungsverhältnis zwischen dem Streben einer Organisation, ihre Umwelt - von der ihr Handeln wiederum selbst gelenkt wird - aufgrund des Bedarfs an Ressourcenzuflüssen zu beeinflussen einerseits und den ebenfalls organisationsintern zu berücksichtigenden Interessen und Forderungen der Mitglieder andererseits (vgl. Schimank 2000: 310 f.). In diesem Zusammenhang ist exemplarisch auf die innerhalb von und mit islamischen Verbänden - zuvorderst DİTIB - existierenden Spannungen und Konflikte hinzuweisen. Für diese ausschlaggebend sind die Abhängigkeit von und Beeinflussung durch den türkischen Staat, die durch spezifische Ereignisse - wie z. B. die sogenannte DİTIB-Spitzelaffäre ${ }^{64}$ im Jahr 2016/2017 - immer wieder auf die innenpolitische Agenda geraten, neue Brisanz erlangen und (finanzielle) Sanktionen zur Folge haben. ${ }^{65}$ In Deutschland bestehen anhaltend politische Forderungen gegenüber DİTIB, vom Herkunftsland ,in Bezug auf strukturelle Verbindungen, Ressourcenbeschaffung, Aktivitäten und Identitätsbildung“ (Rosenow-Williams 2012: 448; Übers.: d. Verf.) politisch und finanziell unabhängig zu werden; dies ist

${ }^{64}$ DİTİB-Imamen soll seitens der türkischen Religionsbehörde Diyanet der Auftrag gegeben worden sein, in Moscheen Informationen über Anhänger der Gülen-Bewegung zu sammeln und diese an die Behörde weiterzuleiten.

${ }^{65}$ Als Konsequenz der Spionage-Affäre kürzte die Bundesregierung die finanzielle Förderung der DİTİB-Zentrale und von DİTİB-Teilverbänden drastisch (BT 2017). 
eine Voraussetzung für den Bezug finanzieller Subventionen sowie die offizielle Anerkennung als vertrauenswürdiger Kooperationspartner.

An dem Fall DİTIB zeigt sich deutlich, wie organisationsinterne und externe Kontextfaktoren in einer komplexen Gemengelage auf die organisationale Performanz einwirken können. Dabei ist von variierenden, situationsbezogenen und personenabhängigen Reaktionen auszugehen. Die Handlungen der Organisationen werden von gegebenen (im-)materiellen und personellen Konstellationen und Abhängigkeiten, politischen Konjunkturen und Entwicklungen im In- und Ausland sowie von Sanktionsmaßnahmen beeinflusst, die bei Nichterfüllung politischer Erwartungen (ad hoc) verhängt werden (können). Organisationen sind demnach nicht als technische Systeme zu verstehen. Abermals wird deutlich, dass es sich bei ihnen vielmehr auch um natürliche, soziale Systeme handelt, die Menschen und Beziehungen umfassen und nicht nach rein technischen Kriterien der Rationalität zu verwalten sind.

In der Betrachtung von Organisationen als soziale Systeme menschlicher Handlungen geraten systemtheoretische Annahmen in den Blick, die das Verhältnis zwischen Organisation und gesellschaftlichem Kontext fokussieren: „Von sozialen Systemen kann man immer dann sprechen, wenn Handlungen mehrerer Personen sinnhaft aufeinander bezogen werden und dadurch in ihrem Zusammenhang abgrenzbar sind von einer nicht dazugehörigen Umwelt" (Luhmann 2005a/[1975a]: 10). Für die soziologische Systemtheorie konstitutiv sind die Grenzziehung und Grenzerhaltung („boundary maintenance“) zwischen System und Umwelt, da sie sich für die Leistung der Komplexitätsreduktion und die Ver- und Bearbeitung für an das System herangetragene Probleme und die Systemerhaltung als zentral erweisen (Luhmann 2012/[1987]: 35 ff., passim) ${ }^{66}$. Die Abgrenzung impliziert eine operative Geschlossenheit der Organisation, durch die sich das soziale System ermöglicht und von der Umwelt unterscheidbar macht. Die Existenzsicherung bedingt jedoch Abhängigkeit, Austausch und Kommunikationsbeziehungen mit der umgebenden Außenwelt und damit eine potenzielle Offenheit der Organisation (Pickel 2017: 384). Den Aspekt der notwendigen Systemgrenzen hat v. a. Niklas Luhmann stark gemacht: „Als System läßt sich [...] alles bezeichnen, worauf man die Unterscheidung von innen und außen anwenden kann; denn in dem Maße, als eine Ordnung sich ausprägt und verdichtet, müssen

\footnotetext{
${ }^{66}$ Vgl. zum sogenannten grenzerhaltenden Systemtyp (,,boundary-maintaining type of system") (Werte, Motive, Handlungssysteme) auch die Ausführungen bei Talcott Parsons (1991/[1951]: 23, Fn 7; 323 ff.). „The definition of a system as boundary-maintaining is a way of saying that, relative to its environment, that is to fluctuations in the factors of the environment, it maintains certain constancies of pattern, whether this constancy be static or moving“ (ebd.: 324; Herv. i. Orig.).
} 
unterscheidende Grenzen gezogen werden, und andererseits setzt die Erhaltung der Grenzen eine darauf abzielende innere Ordnung voraus“" (Luhmann 1964: 24; Luhmann 2005a/[1975a]: 10 ff.). Daraus resultieren unmittelbare Anforderungen an das soziale System Organisation: Mit dem 1.) Bestands- oder Effizienzproblem, d. h. der notwendigen Ressourcengewinnung aus der Umwelt, verknüpft sind 2.) die Herausforderungen der Anpassung durch Einflussnahme auf die Umgebung und Konvergenz mit bestehenden Ansprüchen sowie 3.) des Aufbaus einer inneren Ordnung, die wiederum nur bewältigt werden können, wenn 4.) das soziale System von seiner Umwelt abgegrenzt ist (Luhmann 1964: 23 ff., 62 ff.; Luhmann 2012/[1987]).

Wie bereits in vorigen Kapiteln aufgezeigt $(4.3 .3$; 4.3.4), grenzen sich formale Organisationen durch die Mitgliedschaft und daran geknüpfte Bedingungen und Verhaltenserwartungen von der Außenumwelt ab (Luhmann 1964: 60). Damit ist neben den Beziehungen einer Organisation zur äußeren Umwelt ihre „Innenwelt" angesprochen (Neidhardt 2017/[1979]: 436; Horch 1983: 16 f., 98 f.), die es durch eine gewisse Flexibilität und Elastizität in den Verhaltenserwartungen auszubalancieren gilt (s. auch Streeck 2017/[1987]: 528 ff.). Laut Luhmann wird die Anpassungsfähigkeit gegenüber der äußeren Umwelt durch eine interne funktionale Differenzierung nach Subsystemen und Stellenrollen gewährleistet (Luhmann 1964: 137 ff.; s. auch Luhmann 2005d/[1975d]: 73 ff.). Eine Kombination von spezifischen und generellen Erwartungen ist demnach sinnvoll, da unter wechselnden Verhältnissen Veränderungen sowohl von speziellen als auch generellen Erwartungen möglich seien (Luhmann 1964: 137 ff.; vgl. auch Luhmann 2005d/[1975d]: 67 ff.; Luhmann 2012/[1987]: 411 ff.). Damit ist eine Ambivalenzstrategie angezeigt, mit der ,die Erwartungsstruktur und die Aufgabenverteilung im System unbestimmt gehalten“ und ,einer rasch veränderlichen Umwelt" (Luhmann 1964: 151) angepasst werden könnten. Anforderungen und ihre Veränderungen, die die Organisation betreffen, werden damit auf die Mitglieder übertragen (ebd.: $151 \mathrm{f}$.).

Eine direkte Einflussnahme auf die Außenumwelt kann bei Betrieben und Verwaltungen zumeist über ökonomische Mittel und rechtliche Verpflichtungen erfolgen (Horch 1983: 103). Um die mit der Abhängigkeit von und Anpassung an die Umwelt verbundenen Schwierigkeiten zu umgehen oder zu verringern, könnten sich (freiwillige) Organisationen gegenüber einer möglicherweise als feindlich wahrgenommenen Umwelt abschotten (Willke 1976: 432) oder eine „kämpferische Außeneinstellung“ einnehmen, um bei fehlender funktionaler Differenzierung, Flexibilität in der Auswahl von Mitteln und Strategien zu sichern (Luhmann 1964: 148). Allerdings entbehrten kämpferische Einstellungen gegenüber der Umwelt der „Voraussehbarkeit und Erfolgssicherheit“ ihrer 
Organisierbarkeit (ebd.: 152; s. auch 376 f.). Vielmehr erscheint es wahrscheinlicher, dass Organisationen versuchen, Beeinflussung und Abhängigkeit von außen durch Erlangung von mehr ökonomischer Selbstständigkeit durch Eigenleistungen zu reduzieren (Horch 1983: 103). In freiwilligen Vereinigungen sind vorhandene Geldmittel allerdings zumeist sehr knapp bemessen und müssen akquiriert werden, um die Handlungsfähigkeit der Vereinigung sicherzustellen. Diverse persönliche Beziehungen der Mitglieder oder bestimmter einzelner Personen können Zugänge zu relevanten gesellschaftspolitischen Bereichen öffnen. Unabhängig davon können eine professionelle Außendarstellung und attraktive Öffentlichkeitsarbeit für organisationale Anliegen mobilisiert und durch alternative Maßnahmen nur schwer ersetzt werden (ebd.: 104).

Jenseits des dauerhaften Bedarfs an finanziellen Mitteln und ihrer notwendigen Beschaffung sehen sich Organisationen mit einer Vielzahl von gesellschaftlichen Teilbereichen konfrontiert, auf deren Funktionslogiken sie einzugehen und deren Regeln und Normen sie zu beachten haben. Martin Petzke und Hartmann Tyrell sprechen in diesem Zusammenhang vom „Phänomen der organisationellen Multireferentialität" (Petzke/Tyrell 2012: 293; Herv. i. Orig.). Diese gelte insbesondere auch für religiöse Organisationen und erkläre deren „organisationsuntypische[n] Ambivalenzen“ sowie Prozesse ihrer „Selbstsäkularisierung“ (ebd.). Mehrfachmitgliedschaften von Religionszugehörigen in auch profanen gesellschaftlichen Teilbereichen, Abwanderungen in andere Subsysteme, Rückgriffe auf andere Entscheidungsmechanismen als der Kontingenzbewältigung und daraus resultierende Grenzüberschreitungen könnten allesamt eine Verschiebung der Handlungsmodi von sich ursprünglich religiös verstehenden Organisationen implizieren und zu einer Ähnlichwerdung bzw. Angleichung von religiösen und nicht-religiösen Vereinigungen führen. Eine solche Beobachtung könne wiederum „Zweifel und Misstrauen an der religiösen Bedeutung" von einer sich in offiziellen Außendarstellungen oder Leitbildern als religiös präsentierenden Organisation und die Beklagung von deren „Selbstsäkularisierung“ seitens gläubiger Mitglieder zur Folge haben (Pickel 2017: 391 f.). Luhmann spricht in diesem Zusammenhang von „,weltlichen Geschäften“ freiwilliger Vereine oder religiöser Gruppen, „die sich um ideale Zwecke scharen“ (Luhmann 1964: 306). Diese hätten allerdings Umwelterwartungen zu befolgen und Anpassungsleistungen zu vollziehen, die nicht mit den eigenen Normen zu rechtfertigen seien, ,aber erfüllt werden müssen, soll der Bestand des Systems nicht gefährdet werden“ (ebd.: 305).

Die organisationale Multireferentialität entspricht einer im besonderen Maße für religiöse migrantische Vereinigungen und deren Aktivitäten ausgemachte Multifunktionalität (4.1; 4.4.2). Die MSO können genuinen herkunftsland- und 
migrationsspezifischen Eigeninteressen nachgehen; zugleich sind für sie aufnahmegesellschaftliche Bedingungen und Umwelterwartungen relevant und $\mathrm{zu}$ befolgen bzw. solche ernst zu nehmen, so sie sich einen legitimen Status und Geldzuflüsse sichern wollen (4.3.2). Zentral ist dabei immer die Abhängigkeit von Ressourcen personeller, ideeller und materieller Art, die auch aus dem Ausland zugeführt werden können. Je nach Selbstverständnis und wahrgenommenem Abhängigkeitsgrad werden Konzessionen gemacht - oder sie unterbleiben. Den in einem separaten Kapitel dieser Arbeit beleuchteten Kernthesen des soziologischen Neoinstitutionalismus zufolge (4.5) orientieren sich Organisationen nicht in erster Linie an zweckrationalen, ökonomischen Effizienzkriterien, sondern an der Sicherung ihrer Legitimität und Anerkennung und damit an in ihren Umwelten bestehenden Erwartungshaltungen.

\subsection{Anreiztheoretische Grundannahmen zum Engagement in Organisationen}

Am Anfang dieses Kapitels steht die zentrale Frage: Warum sind Menschen bereit, sich mit ihren Leistungen in eine Organisation einzubringen, insbesondere, wenn dies auf freiwilliger Basis und ohne Bezahlung geschieht? Ihrer Beantwortung soll in diesem Kapitel näher auf den Grund gegangen werden.

Anreiztheoretische Ansätze gehen davon aus, dass alle Arten von Organisationen Individuen bestimmte Stimuli offerieren müssen, um diese dazu zu bewegen, sich in den Dienst stellen bzw. mit bestimmten Aufgaben betrauen zu lassen (Simon et al. 1950; Clark/Wilson 1961; Barnard 1970/[1938]; Simon 1981/[1945]; March/Simon 1976/[1958] ${ }^{67}$; Becker 1982; Scott 1986; Wiepking/Bekkers 2010). Die organisationalen Anreize und subjektiven Beweggründe der Individuen fallen dabei vielfältig aus. Sie können subtil oder offensichtlich, greifbar und nicht greifbar sein bzw. durch das Individuum bewusst oder unbewusst wahrgenommen werden. Zudem variieren sie nach Organisations- und Mitgliedertypen und sind im Kontext des kooperativen organisationsinternen Handelns zu betrachten. Subjektive Motive für individuelles Handeln (4.3.3.2) und organisationale Anreize sind nicht a priori und automatisch deckungsgleich, da sie verschiedenen Einflussfaktoren unterliegen. Sie sind als miteinander korrespondierend und sich gegenseitig komplementierend $\mathrm{zu}$ betrachten. Es ist davon auszugehen, dass sie dynamisch

${ }^{67}$ Die Zahlen in den eckigen Klammern beziehen sich auf die englischsprachigen Erstausgaben der Publikationen. Bei den anderen handelt es sich um die Erscheinungsjahre der deutschen Übersetzungen. 
sind und sich im Rahmen freiwilliger Kooperationszusammenhänge prozessual angleichen (Etzioni 1975: 12 ff.; Willke 1978: 354 f.; Horch 1983: 31, 67). Zudem können Motive auch durch die Organisation und ihre Sozialisationsfunktion und den dort stattfindenden Interaktionsformen beeinflusst werden (Barnard 1970: 49, 129-132; March/Simon 1976: 52 f.; Nadai 1996: 89 ff., Wilson 2000; Gazley 2012: 1246).

Im Folgenden sollen das Anreiz-Beitrags-Modell der zum „Grundinventar der Organisationsforschung" (Preisendörfer 2016: 139) gehörenden verhaltenswissenschaftlichen Entscheidungstheorie ${ }^{68}$ und weitere in Bezug auf den Forschungsgegenstand dieser Arbeit grundlegenden Annahmen zu organisationsinternen Prozessen dargelegt werden. Den Ausgangspunkt bilden dabei die zuerst von Chester Irving Barnard systematisch angestellten Überlegungen, die insbesondere von Herbert Alexander Simon und James G. March weiterentwickelt wurden (Barnard 1970/[1938]; Simon 1981/[1945]; March/Simon 1976/[1958]).

Das verhaltenswissenschaftliche Anreiz-Beitrags-Modell scheint für eine Anwendbarkeit auf die in dieser Arbeit relevanten freiwilligen migrantischen Vereinigungen besonders geeignet zu sein, da es von der möglichen Existenz einer Vielfalt von Anreizen ausgeht und sich gegenüber rationalen Kosten-NutzenKalkulationen sowie einer Dominanz ökonomischer Belohnungen skeptisch zeigt. Daher sollen im Folgenden seine Kernargumente sowie die für die Verfügbarkeit der Anreize bzw. die als organisationale Einbindungsmittel wirksamen wesentlichen gruppensoziologischen Aspekte und Mechanismen beleuchtet werden, die aus sozialen Interaktionsprozessen und der Einflussnahme durch die Gruppe erwachsen. Unter Bezugnahme auf Gedankengänge von auch jenseits der verhaltenswissenschaftlichen Entscheidungstheorie zu verortenden Vertretern von Anreiz-Modellen (Clark/Wilson 1961) soll dargelegt werden, worin diese Anreize der Beitragsleistung für das Individuum in unterschiedlichen nicht-religiösen und religiösen Organisationen - insbesondere freiwilligen Vereinigungen (Horch 1983) - konkret bestehen können und welche „Methoden“ sich die Organisation für die Bereitstellung der Anreize zunutze machen kann (4.4.1).

Der theoretische Grundstock des Anreiz-Beitrags-Modells soll den Rahmen bilden für die darauffolgende Darlegung von religionssoziologischen Hypothesen zu vorteilhafte(re)n Bedingungen für die Mobilisierung von Engagement in religiösen Organisationen (4.4.2). Der sittlich-moralische Imperativ der verhaltenswissenschaftlichen Entscheidungstheoretiker, der vereinzelt auch auf religiöse

${ }^{68} \mathrm{Zu}$ den Protagonisten gehören Chester Irving Barnard (1886-1961), Herbert Alexander Simon (1916-2001), James G. March (1928-2018), Richard Michael Cyert (1921-1998) und Johan Peder Olsen (*1939). 
Aspekte Bezug nimmt, wird damit durch religionssoziologische Lehrmeinungen komplementiert.

Abschließend sollen die potenziellen Vorteile bei der Gewinnung von Freiwilligen in religiösen gegenüber nicht-religiösen migrantischen Zusammenschlüssen im Migrations- und Aufnahmekontext unter Berücksichtigung empirischer Befunde reflektiert werden, um zwei im empirischen Teil dieser Arbeit zu überprüfende Hypothesen aufzustellen.

\subsubsection{Das Anreiz-Beitrags-Modell als verhaltens- wissenschaftliche Entscheidungstheorie}

Die verhaltenswissenschaftliche Entscheidungstheorie beschäftigt sich in erster Linie mit von Individuen oder Gruppen in Organisationen getroffenen Entscheidungen, deren Analyse die Organisationsprozesse und -strukturen erklären und verstehen helfen. Sie zählt zu den einflussreichsten Organisationstheorien. Des Weiteren hat sich die Theorie mit Entscheidungen bzw. Motiven von Personen befasst, einer Organisation beizutreten, sich in dieser aktiv einzubringen, dort zu verweilen oder dieses eben nicht zu tun. Zentral ist in diesem Zusammenhang die von Chester Irving Barnard (1970: 122-138) begründete Theorie der „Ökonomie der Anreize" bzw. des Anreiz-Beitrags-Gleichgewichts, mit der die Bindungsund Überlebensfähigkeit oder der Zerfall einer Organisation als Kooperationssystem erklärt wird (siehe dazu auch Simon 1981: 141-152; March/Simon 1976: 81-105).

Menschen werden einerseits als egoistische Wesen betrachtet, die ihre Motive und Ziele innerhalb eines spezifischen Kontextes realisieren wollen, der unter Umständen nicht die optimalsten Bedingungen oder mehrere alternative Optionen für die Verwirklichung der Ziele bereithält (Barnard 1970: 12, 122). Andererseits wird angenommen, dass Menschen mit biologischen Unzulänglichkeiten ausgestattete soziale Geschöpfe und daher auf Interaktionen und Kooperationen mit anderen Individuen angewiesen sind, von denen sie im starken Maße profitieren können (ebd.: 21 ff., 31 ff., 61). Barnard zufolge ist eine Einbindung in „ein System bewußt koordinierter Handlungen oder Kräfte" (ebd.: 76; Herv. weggel.) effektiver als das Handeln im Alleingang.

Die Grundannahme des Anreiz-Beitrags-Modells besteht darin, dass Organisationen für ihren Bestand auf das Einverständnis von Personen angewiesen sind, Beiträge zu leisten. Die Bereitschaft von Menschen zur Zusammenarbeit und Leistungserbringung kann in allen Organisationstypen durch die Bereitstellung einer Vielzahl von angemessenen Inzentiven erreicht werden (ebd.: 122). Es ist 
konstitutive Aufgabe der Organisation, den beteiligten Individuen kontinuierlich Anreize zu bieten, die sie motivieren und befriedigen und die eine Gegenleistung für ihr Engagement in der Organisation darstellen. Eine Person wird sich zugunsten einer Kooperation entscheiden, wenn der persönlich erwartete Nutzen bzw. der Reingewinn an persönlicher Befriedigung durch Anreize die Kosten der Teilnahme übersteigt oder Anreize die Kosten mindestens ausbalancieren (Barnard 1970: 48, 59, 61 f., 78, 80 f., 122 f., s. auch Simon et al. 1950: 382; Clark/Wilson 1961; Becker 1982: 4; Wiepking/Bekkers 2010: 4). „Solange der einzelne seine Motive durch das befriedigt sieht, was er tut, solange wird er kooperieren; andernfalls hört er auf" (Barnard 1970: 59). Die Zufriedenstellung der Beteiligten sichert den Bestand der Organisation: „Insofern sind Organisationen abhängig von den Motiven der Individuen und den Anreizen, die sie zufriedenstellen" (ebd.: 81). Einschätzung und Erwartung der persönlichen Zufriedenheit seitens der Beteiligten bilden sich dabei auch durch den Vergleich mit wahrgenommenen alternativen Mitwirkungsoptionen in anderen Organisationen aus (ebd.: 80). Vor dem Hintergrund von Wahlmöglichkeiten kann ein negativ bewertetes Verhältnis zwischen subjektiven Beiträgen und Anreizen den Willen bestärken, die Organisation zu verlassen. Gegenüber außerorganisationalen, potenzielle Konkurrenz darstellenden Angeboten kann eine Organisation zwei Strategien verfolgen: Die Stärke der Vorteile erhöhen oder das Ausmaß der Nachteile verringern (March/Simon 1976: 122 f.). ,Eine Verbesserung des Verhältnisses zwischen Anreiz- und Beitragsnutzen vermindert die Neigung des individuellen Teilnehmers, aus der Organisation auszuscheiden[,] während eine Verschlechterung dieses Verhältnisses eine gegenteilige Wirkung zur Folge hätte“ (ebd.: 89; Herv. weggel.; vgl. auch Hirschman 1970; 4.2.3).

Das damit implizierte Prinzip der individuellen Kosten-Nutzen-Abwägung scheint zunächst mit ökonomischen Theorien rationalen Handelns bzw. einem rationalen Systemverständnis übereinzustimmen. Allerdings ist herauszustellen, dass der von Barnard gewählte Begriff der Ökonomie nicht allein auf monetäre bzw. materielle Aspekte zielt (Barnard 1970: 132). Denn Barnard ist grundsätzlich der Auffassung, dass ökonomische Anreize für die Leistungsfähigkeit einer Organisation - im Sinne ihrer Fähigkeit, die Mitwirkung von Personen zu sichern - allein nicht ausreichen (ebd.: 86, 125). Zudem kritisiert Barnard die Überbetonung des in den 1950er Jahren in der neoklassischen Ökonomie vorherrschendem Konzepts des „homo oeconomicus“ (ebd.: 7 f.) und unterstreicht die immateriellen, zwischenmenschlichen, informellen und moralischen Grundlagen von Kooperationsbereitschaften. Dieser ,moralische Imperialismus“ Barnards (Scott/Davis 2007: 72) stellte in dieser Form ein auch in der Folgezeit einflussreiches Novum dar. Noch bedeutsamere Auswirkungen hatte Barnards Ansatz, 
den Individuen aufgrund physikalischer, biologischer und sozialer Faktoren sowie ihrer erfahrungsbedingten Konditionierung eine lediglich begrenzte Fähigkeit zu freien Entscheidungen zuzusprechen (Barnard 1970: 44; 32 f., 61). Herbert Alexander Simon (1981) baute diese Überlegungen Barnards zum Konzept der kognitiv begrenzten Rationalität (,bounded rationality“) aus. Bei diesem wird von Akteuren ausgegangen, die aufgrund kognitiv eingeschränkter Möglichkeiten der Aufnahme und Verarbeitung von Informationen und aufgrund von Unsicherheiten hinsichtlich der Konsequenzen von (alternativ möglichen) Entscheidungen nur eingeschränkt rational handeln (vgl. dazu 4.3.3.2, Fn 49). Als Gegenentwurf zum vollständig informierten und rational agierenden Homo oeconomicus entwickelte Herbert Simon (1981: 31, 79 f.) den mit begrenzter Rationalität ausgestatteten „homo organisans“ (s. auch March/Simon 1976: 129 ff.). In diesem Zusammenhang bedeutsam war ebenfalls die Annahme, dass sich Menschen durchaus bemühen, möglichst rational zu handeln, zugleich aber auch versuchen, Entscheidungssituationen für sich zu vereinfachen, indem sie auf eine umfassende Informationsbeschaffung verzichten, sich an Routinen und bewährten Praktiken orientieren und sich auch mit der Erfüllung von Mindestansprüchen zufriedengeben (engl.: „Satisficing“) (Simon 1981: 31, 79 f.; March/Simon 1976: 129 ff.; 4.3.3.2).

\section{„Methode der Anreize“}

Barnard zufolge (1970: 124) kann eine Organisation bei der Ausgestaltung von Anreizen auf die „Methode der Anreize“ zurückgreifen. Je nach Art der Organisation und mit unterschiedlicher Gewichtung kann es sich um folgende Inzentive handeln (ebd.: 124-129):

(1) materielle Anreize, in der Form von Geld oder Gütern;

(2) persönliche, nicht-materielle Aspekte wie Auszeichnungen, Prestige, Rang, persönliche Macht;

(3) angenehme physische Umstände und Arbeitsbedingungen;

(4) ideelle Befriedigungen persönlicher, nicht-materieller, altruistischer Ideale und Werte (z. B. Stolz auf die eigene Arbeit, Loyalität zur Organisation, patriotische Gesinnung, ästhetische oder religiöse Gefühle, selbstloses Engagement für andere oder soziale Zielsetzungen);

(5) die Attraktivität der Zugehörigkeit zu einer Organisation durch als angenehm wahrgenommene homogene soziale Gegebenheiten und eine einhergehende soziale Verträglichkeit, die auch die (in-)formelle Kommunikation erleichtern 
(z. B. aufgrund einer ähnlichen sozialen Herkunft, eines übereinstimmenden Bildungsniveaus, gleicher nationaler oder religiöser Zugehörigkeiten oder Sitten, Gebräuche, Ambitionen);

(6) die Chance, sich als Teilhaber großer, bedeutsamer Ereignisse und Teil wichtiger kooperativer Leistungen zu fühlen oder an der Erreichung eines hoch bewerteten Ziels beteiligt zu sein, was nicht zwangsläufig mit dem Streben nach persönlicher Auszeichnung und Prestigegewinn zusammenhängen muss; (7) das Erleben von Gemeinsamkeiten, Gemeinschaft, Zugehörigkeit, sozialer Sicherheit, Kameradschaft und gegenseitiger Unterstützung in persönlichen Belangen; ,das Gefühl persönlichen Wohlbehagens in sozialen Beziehungen, das auch als Solidarität oder als soziale Integration oder als Herdeninstinkt [...] bezeichnet wird" (ebd.: 128) und das für die Funktionsfähigkeit der formellen Organisation essenziell ist.

Diese Auflistung hat keinen Anspruch auf Vollständigkeit; die Anreize ließen sich auch mit anderen Begrifflichkeiten beschreiben oder noch weiter ausführen. So könnten weitere materielle Anreize herausgehoben werden: die Inanspruchnahme von durch die Organisation zur Verfügung gestellten Leistungsangeboten - wie Weiterbildungen, Freizeitangebote, Versicherungs- oder Rechtsschutz oder auch die Interessenvertretung durch die Organisation nach außen sowie sich durch die Einbindung für das Individuum ergebende vorteilhafte Beziehungen oder Geschäftsvorteile.

Wie bereits angesprochen wurde, ist immer zu bedenken, dass verschiedene Organisationstypen unterschiedliche Motive ansprechen und verschiedene Arten von Anreizen bieten. Demzufolge nehmen Peter B. Clark und James Q. Wilson (1961) eine Unterscheidung von drei Arten von Organisationen als Ausgangspunkt für die Bestimmung und Zuordnung von materiellen, solidarischen und zweckbestimmten Anreizen vor (vgl. 4.3.1). Sie differenzieren zwischen 1.) utilitaristischen bzw. nutzenorientierten Organisationen, wie z. B. Wirtschaftsunternehmen, 2.) solidarischen Zusammenschlüssen, zu denen sie soziale Einrichtungen wie serviceorientierte Wohlfahrts- bzw. Freiwilligenverbände, Schulen und Universitäten zählen, sowie 3.) zweckgerichteten oder ideologischen Organisationen, die nicht trennscharf von den solidarischen abzugrenzen sind, sich von letzteren aber durch eine ausgeprägte weltanschauliche oder religiöse Wertebasis unterscheiden (Clark/Wilson 1961: 137-152; vgl. auch Klöckner 2016: 189 ff.; 4.3.1).

Um Hypothesen über charakteristische Konditionen und Verhaltensweisen in den drei Organisationstypen zu formulieren, ordnen sie diesen idealtypisch drei Arten von Anreizen zu, die in den Zusammenschlüssen jeweils dominant zum 
Einsatz kommen (Clark/Wilson 1961: 134-137). Bei von utilitaristischen Organisationen gebotenen materiellen Anreizen handelt es sich demnach um greifbare (monetäre) Entlohnungen. Solidarische Anreize sind in solidarischen Organisationen verfügbar, grundsätzlich immateriell und können stark variieren. Sie umfassen wahrgenommene Belohnungen wie Kontaktpflege, Interessengleichheit, Geselligkeit und Spaß, die Zugehörigkeit zu und Identifikation mit der Gruppe, den sich aus der Mitgliedschaft ergebenden Status sowie die Aufrechterhaltung von sozialen Unterschieden. Zweckgerichtete oder ideologische Zusammenschlüsse offerieren zielbestimmte Anreize, die wie die solidarischen Inzentive nicht greifbar sind und die sich im Wesentlichen von den angestrebten überpersönlichen, moralisch oder ideell fundierten Organisationszielen ableiten. Die den Zielen innewohnende Eigenwertigkeit oder Würde motiviert Individuen zur Mitwirkung in der Organisation, deren Einheit und Geschlossenheit im Wesentlichen durch das Instrument der Ziele hergestellt wird (ebd.: 146).

Im Kontext der Einteilung von Organisationen in Typen ist der spezifische Charakter freiwilliger Vereinigungen herauszustellen: In diesen stehen keine ökonomischen oder materiellen, sondern vielmehr personengebundene, von einzelnen Handlungen unabhängige soziale Anreize sowie persönliche und symbolische Belohnungen im Vordergrund (Schafer/Klonglan 1974: 203). Im Allgemeinen kann differenziert werden zwischen immateriellen Inzentiven, die an das Mitglied und andere Personen, Gruppen und deren Wechselbeziehungen in der Vereinigung gebundenen sind, und dem damit kontrastierenden materiellen Motivationsmittel der Geldzahlung, das u. a. in Betrieben eingesetzt wird.

Wie auch bei den im Rahmen der vorliegenden Arbeit fokussierten Engagierten mit einem Zusammenspiel von mehreren individuellen Motiven - mit einem „Motivbündel“ - für die Übernahme einer freiwilligen, unbezahlten Tätigkeit zu rechnen ist (4.3.3.2), ist auch bei den Anreizen von einer großen Vielfalt auszugehen: Sie treten in zahlreichen Kombinationsmöglichkeiten auf und besitzen für verschiedene Menschen und Mitgliedertypen in Abhängigkeit vom Grad ihrer Involvierung unterschiedliche Bedeutungen. Zudem können sich Motive und Interessen der Kooperationsteilnehmer durch Interaktionen auch verändern (Barnard 1970: 61). Für aktiv mitarbeitende Personen, die ein bestimmtes Amt innehaben und die an spezifische Verpflichtungen und Regeln gebunden sind, dürften andere Anreize relevant sein als für lediglich sporadisch oder gar nicht Mitwirkende. Demnach werden Organisationen nicht imstande sein, alle für kooperative Leistungen relevanten Anreize in adäquater Weise bereitzustellen (ebd.: 129). 


\section{Erläuterung von immateriellen Anreizen (,Belohnungen“) und Einbindungs- mechanismen}

$\mathrm{Zu}$ den immateriellen Anreizen (,Belohnungen“) und Einbindungsmitteln zählen 1.) Prestige, 2.) Anerkennung, Dank, Vertrauen, Achtung, Rang, 3.) Objekte der Identifikation, 4.) Organisationsziele, 5.) Führungspersönlichkeiten, 6.) Sozialisation und soziale Interaktionsprozesse, 7.) Gemeinschaft und kollektive Normen sowie 8.) Einflussnahme der Gruppe. Die genannten Aspekte umfassen die persönliche Bedürfnis- und Interessenbefriedigung, die Erhöhung der Selbstwirksamkeit und des Selbstwertgefühls, wenn an sich selbst gestellte Anforderungen realisiert werden können.

Diese Anreize sind teilweise bereits angesprochen worden. Sie können nicht von der Organisation allein produziert werden, sondern ergeben sich vielmehr im Rahmen sozialer, kommunikativer Interaktionen (4.3.4). Allerdings kann die Vereinigung dafür sorgen, dass derlei Interaktionsformen, aus denen die sozialen Inzentive erwachsen können, überhaupt möglich sind und gezielt gefördert werden. Die von Horch (1983: 38-50) und anderen Autoren geltend gemachten Anreize und die für den Einschluss des Individuums in die Organisation wesentlichen Gesichtspunkte sollen im Folgenden näher erläutert werden.

(1) Prestige im Sinne von persönlichem Ansehen und Geltung kann durch die Übernahme von bestimmten oder mehreren Aufgaben erlangt werden, aber auch durch besondere Auszeichnungen. Denkbar ist der Fall, dass die Ausführung bestimmter Funktionen aufgrund spezifischer Auswahlkriterien wie überdurchschnittlicher Leistungen oder fachlichem Wissen erfolgt. Auch durch ein gutes Image der Organisation in der Außenumwelt, das z. B. durch ihre Ziele, Mittel, Auswahlkriterien oder Gemeinnützigkeit bedingt sein kann, kann ein Mitglied Prestige erlangen.

(2) Anerkennung, Dank, Vertrauen, Achtung, Rang ergeben sich im Rahmen von auch emotional begründeten dauerhaften sozialen und kollegialen Beziehungen bzw. Tauschprozessen (s. auch Luhmann 1964: 314 ff., 334; Blau 1964: 88 ff.). Für deren Realisierung hat eine Organisation die Rahmenbedingungen zu schaffen. Die Einbindungsmittel der Anerkennung und des Rangs stellen Belohnungen dar, die dem Individuum Einflusschancen und Handlungsfreiheit für seine durch andere Organisationsmitglieder bestärkte Selbstdarstellung einräumen (Luhmann 1964: 157 ff., 348 f.).

(3) Objekte der Identifikation können Organisationsziele (s. u.), die Gruppe, bestimmte Einzel- oder Führungspersonen sowie Tätigkeiten und Ämter sein. Wie bereits in dem Kapitel zu den Dimensionen formaler Organisationsstrukturen (4.3.4) dargelegt sind Ämter und Aufgaben in freiwilligen Vereinigungen häufig gering formalisiert, standardisiert und zentralisiert. 
Dadurch werden dem Individuum bzw. Rolleninhaber zumeist viel Handlungsund Interpretationsspielraum und die Einbringung von Eigenleistungen eingeräumt, was die Entwicklung von Ich-Identität und Selbstdarstellung ermöglicht. Die Gelegenheit, sich eine Position aus einer inneren Überzeugung heraus zu einer eigenen Sache zu machen, kann dazu führen, dass ihre Übernahme als Belohnung oder innerer Anreiz wahrgenommen wird (Horch 1983: 46).

(4) Im Kapitel zu den Organisationszielen (4.3.1) wurde herausgestellt, dass der Identifikation der Mitglieder mit den Zielen in freiwilligen Vereinigungen eine motivierende Funktion zukommt. Die Ziele begründen die auf Freiwilligkeit beruhende Entscheidung zur Mitgliedschaft und unbezahlten Mitarbeit und sie stellen damit einen zentralen Anreiz dar. Dies ist insbesondere dann der Fall, wenn die Realisierung eines Organisationsziels ,selber zur Quelle persönlicher Befriedigung“ und ,zum einzigen oder auch nur zum wichtigsten individuellen Ziel“ (ebd.: 83; Herv. i. Orig.) wird. Dieses kann v. a. in religiösen, patriotischen oder politischen Organisationen der Fall sein (Simon 1981: 61, 143), in denen Ziele dann als direkte Anreizmittel fungieren, die persönliche Bedürfnisse befriedigen können (4.3.3).

Für die Zusammenarbeit bedarf es eines Einheit stiftenden Ziels, um Mitgliedern und Engagierten den Sinn ihres Handlungsbeitrags sowie die Funktionsfähigkeit des Kooperationssystems zu verdeutlichen. Aufgrund dessen seien insbesondere Führungspersonen bemüht, die Existenz eines gemeinsamen Handlungsziels zu vermitteln und die Überzeugung von diesem zu stärken (Barnard 1970: 82). Herbert Simon (1981: 145) knüpft an Barnards Überlegungen an und konstatiert, dass ein andauerndes individuelles Engagement entsprechend der Zielsetzung der Organisation die Loyalität gegenüber der Organisation und ihren Zielen verstärkt (Simon 1981: 145).

(5) Insbesondere in wenig formalisierten Organisationen kann Führungspersonen eine gewichtige, motivierende Rolle für die Mitgliedschaft und eine Vorbildfunktion zukommen (Barnard 1970: 187 ff., 213 ff.; Etzioni 1975: 191-228; Horch 1983: 149). Wie Organisationsziele können sie das kollektive Handeln der Mitglieder inspirieren und ,zur Ausbildung und Erhaltung von anerkannten Verhaltenserwartungen beitragen“ (Luhmann 1964: 207). Die Führung kann als „ein funktionales Äquivalent zur Institutionalisierung von Normen“ (ebd.) fungieren, indem sie Verantwortung übernimmt, Entscheidungen trifft, die Vereinigung repräsentiert (ebd.: 172 ff.) und die Atmosphäre des Miteinanders und Arbeitens prägt (Horch 1983: 106). Die persönliche Autorität informaler Führer, die ihren Einfluss nicht qua Amt, sondern aufgrund besonderer Fähigkeiten oder einer besonderen Ausstrahlungskraft geltend machen können, besitzt expressive und emphatische Züge. An dieser Stelle ist an den Typus des charismatischen Machtinhabers zu denken, der über besondere als vorbildlich betrachtete Fähigkeiten und Eigenschaften verfügt, die ihn in die Lage versetzen, „diffusen 
Einfluss auf die normativen Orientierungen anderer Handelnder auszuüben“ (Etzioni 1975: 334; Übers.: d. Verf.). Amitai Etzioni bezeichnet Charisma als die „Kernenergie von normativen Organisationen“ (ebd.: 218). Manche Personen sind aufgrund ihres Auftretens und Kommunikationsstils imstande, einen großen Einfluss auf Organisationsmitglieder auszuüben und werden aufgrund ihrer besonderen Ausstrahlung als Führungspersönlichkeit und Autorität akzeptiert, deren Anweisungen daher Folge geleistet wird (Simon 1981: 157, 160; s. auch Weber 1985/[1922]: 124). In freiwilligen Vereinigungen sind neben den instrumentellen Aufgaben der Führungsfigur damit auch die expressiven Elemente von Bedeutung, die nicht auf die Erreichung eines konkreten Ziels abheben, sondern die sofortige emotionale Bedürfnisbefriedigung innerhalb der sozialen Beziehungen ermöglichen (Horch 1983: 89, 106, 134).

(6) Die Sozialisation in der Vereinigung betrifft die Einbindung des Individuums in die Organisation durch dessen Aktivitäten und durch regelmäßige und dauerhafte soziale Interaktionsprozesse der Organisationsmitglieder, die bei einem gewissen Intensivitätsgrad und zeitlichem Umfang das Hineinwachsen des Individuums in die freiwillige Vereinigung bedingen. Häufig werden im Rahmen dieser Beziehungen und Kommunikationen eine längerfristige Einbindung bewirkende Werte und Motive erst erschaffen (Homans 1968: 124). „Je mehr Zeit gemeinsam verbracht wird, und je größer die Gruppenkohäsion ist, desto größer ist die Sozialisationskraft der Vereinigung auf die Teilnehmer" (Horch 1983: 47; vgl. auch Etzioni 1975: 272 ff.; Homans 1967: 177) und desto mehr Möglichkeiten ergeben sich für die Gruppe, das Verhalten ihrer Mitglieder zu kontrollieren (s. dazu auch 4.2.5; 4.3.4). Auch Barnard stellt die hohe Bedeutung der sozialen Interaktionsprozesse heraus und betont, dass ,,[d]ie wesentlichen Befriedigungen oder Enttäuschungen, die aus der Teilhabe an einer Zusammenarbeit resultieren [...] sozialer Natur, das Ergebnis der Wechselbeziehungen zwischen den kooperierenden Personen“ und auf „die soziale Konditionierung aller Beteiligten“" zurückzuführen sind (Barnard 1970: 49). Kontinuierliche (informelle) Interaktionen und Beziehungen in Organisationen festigen gemeinsame Normen, Ideale, Orientierungen und Gebräuche als Gemeinschaft und Solidarität stiftende Elemente (ebd.: 104 ff.). Die zwischenmenschlichen Beziehungs- und Kommunikationsformen resultieren dabei aus persönlichen Bedürfnissen und einem „Herdeninstinkt“ (ebd.: 128). Der innere Zusammenhalt der Gruppe stellt als solcher eine Anziehungskraft dar (Homans 1967: 175), die umso größer ausfällt, je stärker das Individuum in der Gruppe verwurzelt ist und je mehr die subjektiv wahrgenommenen Belohnungen aus verschränkten Beziehungen ohne Kommunikationsbarrieren resultieren (March/Simon 1976: 63 ff.; Etzioni 1975: 280 ff.). 
(7) Der über Gemeinschaft wirkende Einbindungsmechanismus betrifft den inneren Zusammenhalt, das Wirgefühl und die auch von Barnard erwähnte für ein gutes (Wert-)Klima sorgende Homogenität der Gruppe. Diese Gruppencharakteristika üben auf die Einzelperson besondere Anziehungskräfte aus. In diesem Zusammenhang bedeutsam sind kollektive Normen, die das Verhalten der Organisationsmitglieder lenken (4.3.4). Diese fungieren als öffentlich und gemeinschaftlich anerkannte Verhaltenskodizes. Das Ausmaß an ihrer öffentlichen Zustimmung bestimmt den Grad ihres Einflusses in sozialen Interaktionen (Barnard 1970: 218). So können etwa die Zehn Gebote der Bibel sowohl in der Gesellschaft als auch innerhalb einer Organisation verhaltensregulierend und hinsichtlich des Zusammenhalts stabilisierend wirken. Kollektive Normen finden auf individueller Ebene in der Moral ihr Äquivalent, die als „privater Verhaltenskodex“ (ebd.: 216) verstanden werden kann. Die vollständige oder teilweise Internalisierung von Normen, $d$. h. verbindlich gemachten Verhaltensregeln bzw. -erwartungen (4.3.3.2), führt zur Ausbildung einer gefestigten inneren sittlichen Haltung, einer persönlichen Moral, die zu einer stark handlungsleitenden Kraft werden kann.

(8) Es sind die kollektiven Normen und die Interaktionen der miteinander kooperierenden Personen, die den Grad der Einflussnahme der Gruppe auf das Individuum und dessen Identifizierung mit letzterer bestimmen. Deren Einfluss fällt umso größer aus, je mehr sich das Individuum mit der Gruppe identifiziert und ist besonders effektiv, wenn die Gruppe eine homogene Meinung hat und von ihr keine antagonistischen Beeinflussungen ausgehen. James March und Herbert Simon (1976: 63-65) zufolge fällt die Neigung des Individuums zur Identifikation mit der Gruppe umso intensiver aus,

- je stärker gemeinsame Ziele wertgeschätzt werden;

- je häufiger soziale Interaktionen zustande kommen;

- je höher die subjektive Bedürfnisbefriedigung in der Gruppe ausfällt;

- je größer das Prestige der Gruppe eingeschätzt wird;

- je weniger Wettbewerb unter den Gruppenmitgliedern herrscht.

In der freiwilligen Vereinigung besitzt die Verquickung von Wirgefühl und persönlicher Mitwirkung also eine bedeutende Rolle. Sie können sich wechselseitig verstärken und zusätzlich gesteigert werden durch weitere in diesem Kapitel thematisierte Anreize, wie z. B. die als angenehm wahrgenommene Homogenität der Gruppe oder den Erhalt von Anerkennung (Übersicht 4.1). In freiwilligen Vereinigungen sind $v$. a. auch emotionale Bedürfnisbefriedigungen und Belohnungen bedeutsam, die sich innerhalb der sozialen Beziehungen ergeben. 
Übersicht 4.1 Organisationale Anreize und Einbindungsmittel: Ein Überblick

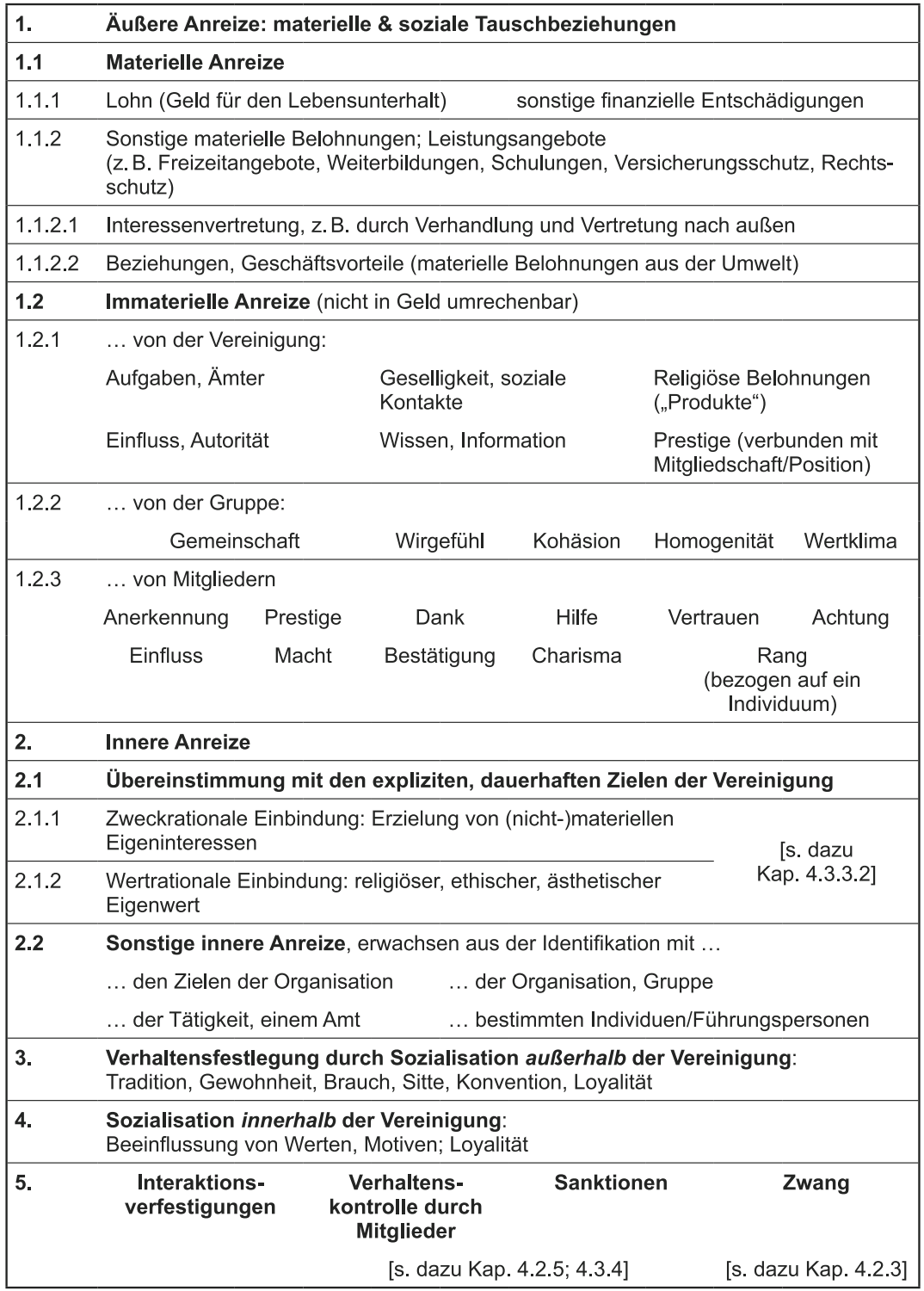

Quelle: Eigene Zusammenstellung nach Horch 1983: 34, Barnard 1970: 124-129. 


\subsubsection{Religionssoziologische und religionsökonomische Hypothesen zur Engagementmobilisierung in religiösen Organisationen}

Was unterscheidet religiöse von nicht-religiösen Vergemeinschaftungen hinsichtlich der von ihnen für Individuen gebotenen Anreize, sich freiwillig in die Organisation einzubringen? Und welche Bindungs- und Einbindungsprozesse kann Engagement in religiösen Organisationen im besonderen Maße stimulieren und ihnen im Vergleich zu säkularen Organisationen potenziell einen Vorteil bei der Rekrutierung von freiwillig Mitwirkenden einräumen? Diese Fragen sollen in diesem Kapitel anreiztheoretisch eingebettet und die dargelegten Annahmen wiederum mit Blick auf ihre Übertragbarkeit auf das Untersuchungsobjekt dieser Arbeit am Ende des Kapitels kritisch reflektiert werden. Die Fragen wurden zum Teil bereits in den Kapiteln zu individuellen Motiven (4.3.3.2) und zum religiösen Sozialkapital thematisiert (4.2.4; 4.2.5), denn motivations-, sozialkapital- und anreiztheoretische Annahmen überlappen sich teilweise und sind entsprechend zusammenzudenken. Daher werden einige Aspekte erneut aufgegriffen. In diesem Kapitel liegt der Fokus allerdings auf dem Passungsverhältnis („Matching“) - der Kongruenz und Interdependenz - von Individual- und Organisationsebene bzw. von individuellen und gemeinschaftlich-zwischenmenschlichen Horizonten und Bedarfen.

Zunächst ist festzuhalten, dass Religion aus der religionssoziologischen Perspektive für das gläubige Subjekt eine bedeutende Form der Kontingenzbewältigung, d. h. der Bearbeitung erfahrener Unsicherheit und Unvorhersehbarkeit darstellt, und damit der Sinnstiftung, Orientierung und Entlastung im Alltag dient (vgl. Pollack 2018: 38 f.; Krech 1999: 22 ff. unter Verweis auf u. a. Luhmann 1977). Eine wichtige verstärkende Funktion kommt institutionellen Strukturen zu: Um im Diesseits Wirkung zu entfalten, müssen religiöse Überzeugungen durch soziale Interaktions- und Kommunikationsprozesse bestätigt und plausibilisiert werden (Berger 1970: 59 ff.; Tyrell 2008: 264 f.). Gottesglaube, religiöse Praktiken wie Rituale, Zeremonien und Gottesdienste - so die Hypothese von Religionssoziologen - sind daher auf die Sozialform Religion, die religiöse Gemeinschaft, angewiesen, um in der Gruppe tradiert, beglaubigt und evident zu werden. In der religiösen Gemeinschaft haben die gläubigen Individuen durch den Austausch mit anderen Mitgliedern die Möglichkeit, religiöse Werte, Normen und Weltansichten bestätigend zu kommunizieren (Wilson 1982: 32 f., 159). Je mehr die Infrastruktur der religiösen Gemeinschaft diese Bestätigung zu leisten imstande ist, desto stärker können sich subjektiver Glaube und religiöse Identität 
ausprägen und umso mehr werden sie zu unhinterfragten Gewissheiten (Berger 1970: 59; Olson 1993: 32).

Es sind also religiöse Organisationen, die den in ihnen eingebundenen Individuen strukturelle Bedingungen und soziokulturelle Realisierungsmöglichkeiten („Gelegenheitsstrukturen“) für gemeinsame religiöse Praxis und freiwillige Tätigkeitsausübung bieten (4.3.3.1; 4.3.3.2). Es ist daher davon auszugehen, dass die Mitgliedschaft und die Beitragsleistung in einer spezifische Normen, Werte, Menschenbilder und Verpflichtungen propagierenden religiösen Vereinigung auf einer bewussten wertegeleiteten, religiös verankerten Entscheidung - einer Selbstselektion - beruhen. Diese ist für die persönliche Lebensführung und die Erfüllung internalisierter Werthaltungen hochbedeutsam und stellt andere Auswahl- bzw. Relevanzkriterien in den Hintergrund.

Auch in nicht-religiösen Zusammenschlüssen können spezifische Wertorientierungen die Basis für das organisationale Selbstverständnis und Handeln darstellen. Allerdings dürften sich religiöse von säkularen Vereinigungen hinsichtlich der umfassenden lebenssinnkonstitutiven und lebensbiografisch integrativen Bedeutung von religiösen Orientierungen und Handlungsdispositionen für die subjektive Beitrittsentscheidung sowie hinsichtlich der Relevanz der inneren, aktiven Beteiligung in der religiösen Gemeinschaft unterscheiden (Smidt 2003c: 218; Roßteutscher 2009: 36).

Die religiöse Wertebasis der religiösen Organisation impliziert, dass ihre Aktivitäten und Angebote bekenntnisorientiert ausgerichtet sind und deren freiwillige, unbezahlte Ausführung oder Inanspruchnahme durch Mitglieder daher als solche Anreize darstellen. Gleiche Konfessionsangehörigkeit bzw. gewisse Affinität zu der von der Vereinigung vertretenen Glaubensrichtung sollten eine Voraussetzung für die aktive Beteiligung darstellen, weil mit dieser Kontakte zu religionsnahen Dienstleistungen und Aktivitäten eingegangen werden. Im Gegensatz zu außerreligiösen Kontexten erfolgen Zusammenarbeit und kollektive Aktionen in religiösen Gemeinschaften häufig auf immateriellen Anreizen, die in religiösen Überzeugungen, wie dem Wunsch gute Werke zu leisten und gottgefällig zu handeln (Smidt 2003c: 218), verankert sind. Dies kann intensives Engagement evozieren und sowohl dem Individuum als auch der Gemeinschaft dienlich sein (Harris 2003: 121 f.; Beckford 1975: 28 f.; 4.2.4). Corwin Smidt (2003c: 218) zufolge werden Kooperationsbereitschaft und hohes Engagement in religiösen Organisationen durch transzendente Werte, Normen der Reziprozität und erwartete Belohnungen forciert - selbst wenn diese erst nach dem Tod im Jenseits eintreten. Denn die Erwartung aufgeschobener Belohnungen im Jenseits durch das Bewusstsein, gute Taten zu vollbringen, göttlichen Willen oder Auftrag zu erfüllen, könne einen stärkeren Anreiz für Reziprozität bieten als weltliche Quellen (Harris 2003: 123). 
Damit ist herauszustreichen, dass religiöse Werte Bewertungsstandards implizieren, die gläubige Individuen danach streben lassen, sich diese Standards in Form religiöser Innerlichkeit und Verbindlichkeit zu eigen zu machen (4.3.3.2). Aufgrund der anhaltenden Motivation, seinen eigenen Wertemaßstäben gerecht zu werden und dem damit einhergehenden Anliegen, sie innerhalb der Glaubensgemeinschaft zu verifizieren, kann das durch religiöse Mittel erzeugte Sozialkapital eine höherwertigere und dauerhaftere Qualität haben als das in nicht-religiösen Kontexten produzierte (Smidt 2003c: 218; 4.2.4). „For those who harbor commitments that are anchored in religious beliefs, religiously endowed social capital reflects a unique set of resources that enhances the possibility for successful cooperation" (Harris 2003: 121 f.).

$\mathrm{Zu}$ erklären ist dies mit dem Phänomen, dass Beziehungen innerhalb religiöser Vereinigungen durch die gemeinsamen religiösen Überzeugungen und Praktiken gefestigt werden (ebd.: 122; 4.2.5). Regelmäßige Kontakte und Vertrauen zwischen den Mitgliedern befördern das Zusammengehörigkeitsgefühl und gemeinsame Ansichten darüber, was gut und wünschenswert ist und welche Ziele zum gemeinsamen Nutzen erreicht werden sollen (Nemeth/Luidens 2003: 109). Damit ist die in religiösen Zusammenschlüssen stattfindende religiöse Sozialisation angesprochen. Sie umfasst die Vermittlung und Verstärkung dauerhaft gültiger Werte und Normen, die Wohltätigkeit und Engagement stimulieren sowie die Vertrauensbildung und die Bindung an die Gemeinschaft befördern (Cnaan et al. 2003: 20, 29; Wuthnow 1996: 9; Meulemann/Beckers 2004: 66 f.). Im Rahmen familiärer religiöser Sozialisation kann Engagementbeteiligung aufgrund intakter Familientraditionen anerzogen und kollektiviert werden und es ist davon auszugehen, dass es sich bei familiärer und organisationaler Sozialisation um sich wechselseitig verstärkende Prozesse handelt.

Das Wechselverhältnis zwischen einer persönlichen religiösen Überzeugung (,conviction“) eines Individuums und dessen Einbindung in eine religiöse Gemeinschaft bzw. Gemeinde (,community“) reflektiert auch Robert Wuthnow (1991: 121-156) in seinem Conviction und Community-Ansatz: Das Zusammenspiel beider Komponenten sei für die Aufnahme eines freiwilligen Engagements wesentlich. Religiöse Orientierungen könnten insbesondere durch die innerhalb der Organisation geteilten und verstärkten religiösen Werte und Normen, die u. a. die Entwicklung eines höheren Maßes an Frömmigkeit bewirkten, wohltätige Aktivitäten für Bedürftige und Benachteiligte befördern (4.2.4). Fiele der gemeinschaftliche Kontext weg, würden solche Tätigkeiten eher unterbleiben (ebd.: 156). Wuthnow stellt damit die Einbindung der gläubigen Person in die religiöse Gemeinschaft als bedeutsam heraus. 
Darüber hinaus beschäftigt sich Wuthnow mit der Frage, ob spezifische religiöse Überzeugungen oder Lehren karitatives Verhalten bzw. Engagement verstärken (ebd.: 128 f.). Er konstatiert, dass alle großen Religionen der Welt ihre Anhänger ermahnten, mitfühlend und fürsorglich zu sein. Vor allem das Christentum betone die Prinzipien der Nächstenliebe und Barmherzigkeit gegenüber Bedürftigen. Verallgemeinernd verweist er auf die Stärke der biblischen Tradition, die eine wichtige Inspiration für formelles und informelles Verhalten von vielen freiwillig engagierten Personen sei, auf die im Laufe ihrer Sozialisation religiöse Lehren eingewirkt hätten (4.3.3.2).

Gleiches gilt für die theologischen Grundlagen anderer Konfessionen. So kann nach islamtheologischer Perspektive von einem aufrichtigen Glauben nur dann gesprochen werden, sofern dieser durch gute Taten und im zwischenmenschlichen Umgang präsent sei. „Beim guten Werk - und allgemein im islamischen Glaubenssystem - wird Gott in den Mittelpunkt des menschlichen Handelns gestellt“, das ,einzig im Namen Gottes [erfolgt], mit dem perspektivischen Ziel, die göttliche Zufriedenheit (riḍā') zu erlangen und dafür den göttlichen Lohn zu bekommen“ (Begić 2014: 55). Die zu erwartenden Belohnungen beziehen sich dabei auch auf ein Leben im Diesseits und beschränken sich keineswegs auf das Jenseits (Koran 16:97, 16:30, 30:44 f.). Insofern erfolgt Hilfeleistung für andere als Ansporn und im eigenen Interesse. Der Glaube bekundet sich im zwischenmenschlichen Handeln, im diesseitigen Leben, in gottesdienstlichen Praktiken, in der Beachtung sozialethischer und moralischer Grundsätze und Verhaltensnormen (Begić 2014: 64).

Vor dem Hintergrund obiger Ausführungen ist davon auszugehen, dass religiöse Traditionen Engagement in einem intensiven Ausmaß befördern. Andererseits könnten Hilfeleistungen für andere Menschen aber auch als eine allgemeine Tugend betrachtet werden, die nicht in irgendeiner religiösen Tradition verwurzelt sind (Wuthnow 1991: 124). Während natürlich auch nicht-religiöse Menschen (aus anderen Quellen gespeistes) Einfühlungsvermögen zugesprochen werden könne, könne laut Wuthnow bei Menschen mit religiösen Überzeugungen allerdings eher angenommen werden, dass sie dauerhaft altruistisch, nach dem Prinzip der Nächstenliebe sowie den grundlegenden religiösen Geboten handelten, ihre genuinen, internalisierten Überzeugungen also auslebten und in die Tat umsetzten (s. auch 4.3.3.2). Dass religiöse Überzeugungen nicht zwangsläufig zu mitfühlendem, altruistischem Handeln anleiteten, stellt dabei ebenfalls eine verbreitete Auffassung dar (ebd.: 123; s. auch 4.2.4; 4.2.5). 


\section{Das Konzept von Belohnungen und Kosten (Stark \& Bainbridge)}

Angesichts dieser sowohl auf das Diesseits als auch auf das Jenseits bezogenen religiösen Referenzrahmen haben Rodney Stark und William S. Bainbridge (1987) ein Konzept von Belohnungen und Kosten ausgearbeitet ${ }^{69}$, das es im Folgenden näher zu erläutern lohnt.

Kosten würden vom Individuum akzeptiert, um Belohnungen zu erhalten, auch wenn diese Belohnungen in ferner Zukunft liegen, in einem nicht überprüfbaren Kontext, der aber keiner Rationalisierung noch eindeutigen Bewertung zugänglich sei (Stark/Bainbridge 1987: 27, 35 f.). Als Kerntheorie für religiöses Engagement wird so ein Konzept erstellt, in dem Kompensatoren, d. h. psychisch wirkende Verschiebe-, Ausgleichs- und Ersatzmittel, die entscheidende Rolle spielen. Wenn Menschen nicht auf schnellem und einfachem Wege stark erwünschte Belohnungen erhalten könnten, verharrten sie in Bestrebungen, um diese zu erlangen und akzeptierten oft Erklärungen, die die Aufgabe von Kompensatoren im Sinne ,immaterieller Ersatzstoffe“ (ebd.: 36) übernähmen. Deren Wert und Gültigkeit seien jedoch nicht empirisch bestimmbar, sondern könnten nur - und ausschließlich durch den persönlichen Glauben angenommen und verifiziert werden. Demzufolge wird etwa das Verlangen nach Unsterblichkeit oder Hoffnungen auf ein Leben im paradiesischen Jenseits über einen Kompensator befriedigt, d. h. mit einer Art immateriellen Versprechen, dass eine Belohnung folgen wird. Verallgemeinernd gesprochen bezieht sich Religion, Stark und Bainbridge zufolge, auf Systeme von allgemeinen Kompensatoren, die auf das Transzendente betreffenden Annahmen beruhten (ebd.: 39).

Bezogen auf das Phänomen des individuellen Engagements für religiöse Organisationen hängt die persönliche Einsatzbereitschaft von der Nettobalance der Belohnungen und Kosten ab, die wiederum bestimmt ist durch den Grad des (empfundenen) Involviertseins (vgl. oben: Barnard 1970: 48, 59, 61 f., 78, 80 f., 122 f., s. auch Simon et al. 1950: 382). Weil Religionen auf ein übernatürliches Reich verweisen, besäßen sie hinsichtlich der Schaffung und Förderung von Kompensatoren einzigartige Fähigkeiten; zugleich hielten die religiösen Zusammenschlüsse für die Individuen aber auch „reale Belohnungen“ bereit (Stark/Bainbridge 1987: 43 ff.). Wie mit dem Anreiz-Beitrags-Modell bereits thematisiert, können Belohnungen z. B. durch Gemeinschaftserfahrung, Freizeitaktivitäten oder die Besetzung

${ }^{69}$ Stark und Bainbridge haben das Konzept im Rahmen ihrer allgemeinen Theorie menschlichen Handelns und insbesondere ihrer Religionstheorie dargelegt. Ihre theoretischen Ansichten weisen einen eklektischen Charakter auf: Sie basieren auf Elementen der strukturellen Sozialpsychologie, der Lerntheorie, der Theorie der symbolischen Interaktion sowie der Tausch- und Rational-Choice-Theorie (Stark/Bainbridge 1987: 6). 
von Führungspositionen mit einhergehendem Status- oder Machtgewinn anvisiert und erlangt werden.

Religiöse Organisationen ähnelten den säkularen insofern, als letztere ebenfalls Plattformen für menschliches Handeln und Interaktionen seien. Jedoch erleichtere die (Normen-)Kontrolle religiöser Organisationen die Überwachung von Austauschverhältnissen, wodurch die individuellen Kapazitäten erhöht würden, um Kompensatoren gegen begehrenswerte Belohnungen einzutauschen (Stark/Bainbridge 1987: 43). Zudem unterschieden sich religiöse Organisationen von anderen sozialen Institutionen deutlich in Hinblick auf Qualität und Glaubhaftigkeit angebotener Kompensatoren, von denen die Organisationen formal geprägt seien (ebd.: 44).

Stark und Bainbridge operationalisieren religiöse Belohnungen und Kompensatoren für religiöse Organisationen (für den US-amerikanischen Kontext). Zu den von diesen potenziell angebotenen Belohnungen zählen sie folgende (ebd.: 46):

1. die Mitgliedschaft, die dem Individuum Status und Ansehen in der Gemeinde verleihe und die Sicherung anderer religiöser Belohnungen ermögliche;

2. die Teilnahme an Gottesdiensten, die neben spezifischen religiösen Bedeutungsgehalten auch gesellschaftliche Ereignisse und Gelegenheiten aufgreifen und interpretieren;

3. die Beteiligung in religiösen Organisationen und den dort stattfindenden vielfältigen Aktivitäten;

4. die Sozialisierung von Kindern im Sinne einer Vermittlung eines kulturellen und moralischen Erbes.

Als Kompensatoren fungieren Stark und Bainbridge zufolge mindestens folgende Aspekte:

1. Religiöse Lehren, die versprechen, die Lasten des Lebens erträglich zu machen, die Führung und Hilfe zur Verfügung stellen und Wiedergutmachung für irdische Leiden im Leben nach dem Tod verheißen;

2. Religiöse Erfahrungen als eine Befreiung von aufgestauten Emotionen und eine Quelle des Vertrauens in die Authentizität von Kompensatoren (z. B. bei Erscheinung einer Vision);

3. Gebet und private Frömmigkeit als Wege der Suche nach göttlicher Hilfe und Führung sowie als Mittel zur Schuldbekenntnis und Trostfindung;

4. Partikularismus oder moralische Überlegenheit als die Gewissheit, dass man sich unter den von Gott Auserwählten befindet und eine elitäre religiöse Identität besitzt. 


\section{„Religiöse Produkte“" und Akkumulation von „,religiösem Kapital“" (Iannac- cone)}

Laurence R. Iannaccone, einer der Hauptvertreter des Rational-Choice-Paradigmas, spricht in seinem Aufsatz „Religious Practice: A Human Capital Approach“ in diesem Zusammenhang von der Herstellung ,religiöser Produkte“ bzw. der Akkumulation von ,religiösem Kapital“. Beide Elemente seien unsichtbar, gleichwohl durch die Aneignung und Kenntnis religiöser Lehren und Traditionen sowie entsprechender religiöser Praxis zu erzeugen. Letztendlich stellen sie für das Individuum Belohnungen für auf Gottes Wohlgefallen gerichtetes Verhalten dar, sofern man sich dieser Belohnungen glaubensmäßig versichern kann (Iannaccone 1990: 299).

Iannaccone betrachtet religiöse Praxis als einen produktiven Prozess, der in Abhängigkeit von verfügbarem Humankapital, Zeit und weiteren Faktoren religiöse Befriedigungen und Belohnungen produzieren könne. Wenngleich religiöse Produkte komplex und weitgehend nicht beobachtbar seien, könnte religiöse Produktion gemessen werden in Form gekaufter Güter (wie Sonntagskleidung oder Transportkosten zum Gottesdienst), in Form von Opfergaben oder Mitglieds- und anderen Geldbeiträgen, mit denen die gemeinnützige Arbeit religiöser Organisationen unterstützt wird. Dazu gehörten auch der Zeitaufwand für Gottesdienste, Gebete, Meditationen, das Lesen religiöser Schriften sowie weitere religiös motivierte Verhaltensweisen und Arbeitsleistungen. ${ }^{70}$

Gemeindemitglieder würden häufig eigene Fähigkeiten und Erfahrungen nutzen, um religiöse Befriedigung zu erhalten. Analog zum Humankapital (oder dem inkorporierten Bildungskapital bei Bourdieu) sei dafür die Akkumulation religiösen Kapitals erforderlich. Dazu gehörten der Erwerb religiösen Wissens, die Vertrautheit mit religiösen Ritualen, die Kenntnis der religiösen Lehre wie auch Freundschaften mit anderen Gläubigen (4.3.3.1). Die Verfügbarkeit dieser Kenntnisse - d. h. der Besitz von religiösem Humankapital - stelle eine wesentliche Voraussetzung für die Produktion und Wertschätzung religiöser Güter dar. So könnten Gottesdienste nicht gewürdigt werden, wenn keine Vertrautheit mit den ihnen zugrunde liegenden Lehren, Traditionen und Ritualen vorhanden sei. Zudem stelle religiöses Kapital ein wichtiges Produkt religiöser Aktivitäten dar, das der Investition in die eigene Person diene. Denn religiöse Akte der Nächstenliebe dienten nicht allein der Unterstützung bedürftiger Zielgruppen, sondern insbesondere auch der moralischen Verbesserung des gläubigen Menschen. Damit einher gingen in fast jeder Religion Erwartungen von besseren Aussichten in einem Leben im Dies- oder Jenseits.

\footnotetext{
${ }^{70}$ Iannaccone wendet die ökonomischen Konzepte der Haushaltsproduktion und des Humankapitals auf unterschiedliche Ebenen religiöser Partizipation an.
} 
Die Gemeinde könne im Laufe der Zeit zu einer wichtigen Quelle religiöser Befriedigung werden und die weitere Teilnahme motivieren. Die Qualität des Zusammengehörigkeitsgefühls innerhalb einer Gemeinde hängt wiederum stark davon ab, was in diese Beziehungen investiert wurde bzw. wird - Bourdieu spricht in diesem Zusammenhang von einer in der Gruppe zu leistenden „Institutionalisierungsarbeit“ (Bourdieu 1983: 192; 4.3.3.1).

Iannaccones Anliegen ist, die Wechselbeziehung zwischen religiösem Kapital und religiöser Beteiligung zu begründen und nachzuweisen. Denn das religiöse Kapital sei sowohl Voraussetzung als auch Folge der meisten religiösen Aktivitäten und erhöhe das Potenzial und Niveau individuellen religiösen Engagements. Andersherum sei religiöse Partizipation das wichtigste Medium wie Mittel, um den Bestand an religiösem Kapital zu erhöhen. Religiöse Aktivitäten führten quasi zur Aneignung von Fachkenntnissen, die wiederum die durch andere religiöse Handlungen erhaltenen Befriedigungen erhöhten (Iannaccone 1990: 299). Die religiöse Erziehung bliebe jedoch die wichtigste Determinante religiösen Glaubens und Verhaltens und wahrscheinlich auch des religiösen Kapitals (ebd.: 309, 313).

Der in den Ausführungen von Stark/Bainbridge und Iannacconne implizierten ökonomisierten Nutzenmaximierung zur Erklärung religiösen Verhaltens ist aus (religions-)soziologischer Sicht entgegenzuhalten, dass der ,unbedingte[] Eigenwert" (Weber 1985/[1922]: 12; Herv. i. Orig.) eines bestimmten religiösen Sichverhaltens im Hier und Jetzt als solcher - unabhängig vom individuellen Erfolg und Profitstreben - weitestgehend unberücksichtigt bleibt. Der Begriff der Kompensationen verweist auf im irdischen Leben zu erlangende Entschädigungen und notwendige Ausbalancierungen; unzureichend mitbedacht wird die Bedeutsamkeit lebenspraktischer Verwirklichung des Glaubens im diesseitigen Leben durch religionskonforme Handlungsweisen. Diese umfasst soziologisierte, ,weichere' Annahmen der Rationalitätsannahme, die ethisch-sittliche Maßstäbe und mit diesen einhergehende Routinen und Rituale als rezeptartige Handlungsfolgen einkalkulieren. Wie in diesem Kapitel (und anderen) dargelegt erfolgt die Realisierung des Glaubens im Diesseits aufgrund einer subjektiven Internalisierung von handlungsrelevanten religiösen Normen - aufgrund einer persönlichen Disposition, die einer intrinsischen Selbstbewertung unterzogen wird (4.3.3.2). Es handelt sich um auf Traditionen und Ritualen fußenden routinisierten Handlungsweisen (,habits“), die kognitiv und normativ gestützten „Skripten“ folgen (4.3.3.2). Persönliches Profitstreben kann folglich konterkariert werden durch die religiösen Ethiken entsprechende Orientierung an den Interessen oder dem Wohlergehen der Gemeinschaft oder anderer Menschen sowie durch resultierendes prosoziales, karitatives Handeln. Insofern funktioniert die religiöse Weltanschauung als solche „wie 
ein Rahmen [,frame'], welcher determiniert, was als ,Kosten“ und was als ,Nutzen" wahrgenommen wird" (Stolz 2008: 150). Die in spezifischen Situationen jeweils vordergründigen religiös konnotierten individuellen Präferenzen und die „Frames" können jedoch nicht vorhergesagt werden, sondern sie variieren; dadurch wird die in den Ausführungen von Stark/Bainbridge und Iannacconne hervortretene eigennutzorientierte Rationalität relativiert. ${ }^{71}$

\section{Potenzielle Mobilisierungsvorteile religiöser Organisationen - Eine prüfende Bilanzierung}

Angesichts der Erörterungen ist ein potenzieller Rekrutierungsvorteil von religiösen Organisationen somit mehrfachbedingt. Gemeinschaftliche Religionsausübung plausibilisiert den individuellen Glauben und ermöglicht die Realisierung essenzieller persönlicher Bedürfnisse. Einbindung und Anerkennung erfährt das Individuum durch gemeinsame religiöse Praxis sowie die Aneignung von religiösem Wissen. Zudem ist ihm die Akkumulation von religiösem Kapital persönlich von Nutzen, weil dadurch gottgefälliges Handeln gewährleistet wird. Dies entlastet die gläubige Person, übernimmt im Alltag sinn- und orientierungsgebende Funktionen und sorgt für positive Verheißungen. Die regelmäßige Teilhabe in der religiösen Gemeinschaft potenziert persönliches Engagement und religiöses Kapital. Dies wird auch durch Erwartungen der Gemeinschaft sowie (Normen-)Kontrolle aus der Perspektive spezifisch religiöser Ethik und Weltanschauung sichergestellt.

Die durch Individuum und Organisation geteilten Werte und Normen erleichtern die Rekrutierung und Anwerbung von Mitgliedern und Freiwilligen, sie stellen eine gewisse Voraussetzung für ihre Gewinnung dar (Harris 2003: $122 \mathrm{f}$.; Clark/Wilson 1961: 135 f., 146 f.; Nadai 1996: 14 f., 65 ff., 157 f., 209; Roßteutscher 2009: 54). Selbstbeteiligung ist ein genuines persönliches Antriebsmotiv. Die Organisation ist wiederum abhängig von den Ressourcen der Mitglieder und Beteiligten. Selbstselektion und Fremdselektion bzw. Freiwilligenrekrutierung können koinzidieren, womit eine Win-win-Situation für das Individuum und die Organisation hergestellt werden kann. Wie eingangs erwähnt sind individuelle religiöse Motive und die von religiösen Organisationen gebotenen Anreize als sich gegenseitig komplementierend zu betrachten (Barnard 1970: 49, 129-132; March/Simon 1976: 52 f.; Nadai 1996: 89 ff.; Wilson 2000; Gazley 2012: 1246).

\footnotetext{
${ }^{71}$ Siehe zur Einschätzung der Leistungen und Grenzen des Rational-Choice-Paradigmas hinsichtlich der Erklärung religiösen Verhaltens sowie zu Lösungsansätzen für eine bessere (religions-)soziologische Anwendbarkeit: Stolz 2008.
} 
An dieser Stelle ist auf die zu Beginn dieses Kapitels aufgeworfene Frage zurückzukommen, inwieweit es legitim erscheint, die oben dargelegten theoretischen Annahmen auf die Situation der MSO im deutschen Kontext zu übertragen. Neben einer allgemein als defizitär einzustufenden empirischen Erkenntnislage, welche Rolle Religion für migrantisches Engagement in Deutschland konkret spielt, soll hier zunächst auf die in der Literatur herausgestellte Multifunktionalität von Moscheevereinen - aber auch anderen religiösen Versammlungs- und Andachtsstätten - hingewiesen werden (Ceylan 2006: 123 ff.; Behloul/Lathion 2007: 201; Ceylan 2008). Häufig fungieren sie als mehrzweckorientierte Dienstleistungszentren, ,die Unterstützung, Beratung und soziale Angebote bereitstellen und das Selbstvertrauen und den Selbstwert von Immigranten und Immigrantinnen stärken“ (Baumann 2016: 111). Damit sind die Sicherstellung von Versorgung sowie der Selbsthilfecharakter der Einrichtungen angesprochen (4.1), zu denen es für die einst zugewanderten Menschen und deren Nachkommen keine Alternativen zu geben scheint (Halm/Sauer 2005: 58 f., 81; Sauer 2011: 154, 223, 256). Die fehlenden Alternativen sind dadurch bedingt, dass allein die Moscheevereine die religiöse Grundversorgung von Mitgliedern und anderweitig Beteiligten sowie die Vermittlung religiöser Traditionen zu übernehmen imstande sind (Ceylan 2008). Darüber hinaus können in ihnen Herkunftskultur und Muttersprache gepflegt sowie gruppenspezifische Interessen artikuliert und diskutiert werden (vgl. auch Handy/Greenspan 2009; Carabain/Bekkers 2010). Zahlreiche formelle Veranstaltungen, aber auch informelle Zusammenkünfte auf dem Terrain der Moschee machen die Moscheegemeinden zu lebendigen Lokalitäten, die sowohl religiöse als auch nicht-religiöse Aktivitäten anbieten, aber daneben auch schlicht Treffpunkte sind.

Diese Feststellung ist relevant, da aufgrund von Außenerscheinungen und externer Wahrnehmungen angenommen werden könnte, dass Aktivitäten und Engagement in Moscheevereinen und Versammlungsstätten anderer Religionen ausschließlich mit religiösen Motiven und organisationalen religiösen Anreizstrukturen zu begründen sein sollten. Eine solche Vermutung scheint nahezuliegen, weil Religion bzw. Religiosität in der Minderheitensituation häufig eine größere Bedeutung zukommt (Baumann 2004; Fuhse 2006; Nagel 2015a, 2018a), und empirische Studien insbesondere für die muslimische Bevölkerungsgruppe eine im Vergleich zur einheimischen deutschen Gesellschaft höhere Religiosität nachweisen (Sauer/Halm 2019: 111; Pollack/Müller 2013: 54; Brettfeld/Wetzel 2007: 16 ff., 138 ff., 262 ff.). Diese ist teilweise mit dem Phänomen einer intakten intergenerationalen Wertestabilität zu erklären (Sauer/Halm 2019: 111; Diehl/Koenig 2009; Nauck 2007; 4.3.3.2). Innerfamiliäre Transmissionsprozesse in Familien mit Zuwanderungsgeschichte können im Generationenverlauf eine anhaltend hohe 
Stabilität von kulturellen Werten und Einstellungen zur Folge haben. Zurückzuführen ist dies auf eine stärkere Beaufsichtigung der Sozialisation der Kinder und Jugendlichen durch die Eltern, auf die Relevanz familiärer Integritätswerte, hoher (Solidaritäts-)Verpflichtung gegenüber der Familie sowie Absicherung gegenüber potenziell eintretenden existenziellen Risiken (Diehl/Koenig 2009). ${ }^{72}$ Hinsichtlich der Annahme einer Dominanz religiöser Motivik wären ebenfalls integrationspolitische Debatten mit dem Fokus auf die Institutionalisierung „des Islam“ in Deutschland und das diesbezüglich selbstbewusste Auftreten und Selbstverständnis muslimischer Akteure und islamischer Vereinigungen ins Feld zu führen. ${ }^{73}$

Die in Bezug auf (religiöse) Motive von Engagierten mit Migrationhintergrund als stark lückenhaft zu bezeichnende Studienlage liefert keine eindeutigen, gleichwohl aufschlussreiche Ergebnisse. So zeigen die Befunde des Religionsmonitors 2017 (Nagel/El-Menouar 2017: 40 ff.), dass sich Muslime mit einem Anteil von 44 Prozent deutlich stärker in der Flüchtlingshilfe engagieren als dies Christen (21\%) und Konfessionslose tun (17\%). Die Zugehörigkeit zum Islam hat einen signifikanten positiven Effekt auf das Engagementverhalten. Unklar bleibt allerdings, welche konkreten individuellen Motive damit verbunden sind und ob die muslimische Konfessionszugehörigkeit ausschließlich oder dominant mit religiösen Beweggründen für das Engagement einhergeht.

Die Ergebnisse der Studie von Jennifer Klöckner (2016) zu den Motivstrukturen der in Wohlfahrts- und Migrantenorganisationen freiwillig Engagierten weisen darauf hin, dass sich Freiwillige in türkisch-islamischen Vereinen nicht ausschließlich aufgrund religiöser Motive engagieren: Diejenigen, die sich aufgrund religiöser Motive engagieren, präferieren für die Ausübung des Engagements einen religiösen Verein (Klöckner 2016: 434). Allerdings könne die Konfessionszugehörigkeit allein nicht als Grund für die freiwillige Arbeit in religiösen Vereinen gewertet werden, „selbst wenn diese starke Effekte auf die Organisationszugehörigkeit hat“ (ebd.). Auf Basis ihrer Analysen vermutet Klöckner, dass religiöse Motive mit kulturellen einhergehen, da sich eine freiwillige Tätigkeit in einem türkisch-islamischen

\footnotetext{
${ }^{72}$ Hintergrund dieses Bedürfnisses ist auch, dass die Herkunftsländer häufig kein wie in Deutschland gesetzlich verankertes Wohlfahrtssystem aufweisen und die soziale Sicherheit und das Wohl der Einzelperson daher durch familiale Kohäsion und Solidarität gewährleistet werden. Familie stellt damit einen Schutzfaktor dar; individuelles Verhalten unterliegt einer stärkeren sozialen Kontrolle (s. dazu Diehl/Koenig 2009: 303 f.).

${ }^{73} \mathrm{Zu}$ nennen sind hier z. B. die Verankerung der Islamischen Theologie als neue Studiendisziplin an deutschen Hochschulen oder die in der Deutschen Islam Konferenz (DIK) erörterten Möglichkeiten der Etablierung islamischer Wohlfahrtspflege. Die Themen zeigen an, dass um Koexistenz verschiedener Infrastrukturen sowie ein islamtheologisch fundiertes Wohlfahrtsverständnis gerungen wird (Ceylan/Kiefer 2016, 2017b).
} 
Verein sowohl auf die ethnische als auch die religiöse Gruppe und die eigene Herkunftskultur beziehe (ebd.: 435). Mit dem Engagement kann damit sowohl dem persönlichen Glauben als auch der ethnischen Herkunft Ausdruck verliehen werden: Religiöse Motive können sich mit kulturellen und sozialen Aspekten koppeln, die z. B. die Bewahrung der eigenen Herkunftskultur, die Kontaktpflege und den Gruppenzusammenhalt in der Aufnahmegesellschaft betreffen (ebd.: 198). Diese Interpretation der Motivkombination korrespondiert mit US-amerikanischen Studien: „Immigrants tend to be drawn to ethnic congregations in a host country, not only to practice their religion, but also to maintain their ethnic identity" (Carabain/Bekkers 2010: 3; s. auch Handy/Greenspan 2009: 957). Für Deutschland und die Motivforschung im Engagementkontext bleibt diese durchaus plausible Aussage jedoch zunächst ,eine Vermutung“ (Klöckner 2016: 435), die weiterer empirischer Untersuchungen harrt.

Darüber hinaus zeigen sowohl die Befragungsergebnisse des Religionsmonitors als auch diejenigen der Studie von Klöckner, dass die Gemeindeanbindung als solche und nicht der regelmäßige Besuch von Gottesdiensten oder Freitagspredigten für die Aufnahme freiwilliger Hilfeleistungen ausschlaggebend ist (Nagel/El-Menouar 2017: 45; Klöckner 2016: 440). Somit bieten die sozialen Zusammenkünfte eine gute Ausgangsbasis, um Personen in die Aktivitäten der Gemeinde einzubinden, und die Bedeutung von Gemeinden als soziale Orte und Engagementplattformen wird deutlich (Nagel/El-Menouar 2017: 29 f., 45). Klassischerweise wird herausgestellt, dass freiwillig Engagierte über persönliche Beziehungen und direkte Ansprachen vor und nach religiösen Veranstaltungen, insbesondere Gottesdiensten, gewonnen werden können (Cnaan/Curtis 2013: 23 f.; Wilson/Janoski 1995: 138 f.; Wilson/Musick 1997: 700 ff.; Wuthnow 1991: 125 f.; Jackson et al. 1995; De Hart/Dekker 2005). Dabei ist jedoch davon auszugehen, dass die Häufigkeit von Gottesdienstbesuchen nicht per se in allen Konfessionen und gesellschaftlichen Kontexten als Indikator für hohes individuelles religiöses Commitment und Eingebundensein in die Glaubensgemeinschaft fungiert - wie es häufig angenommen wird (s. dazu auch Smidt 2003c: 217 f.; Klöckner 2016: 440 f.). Insofern liegen empirisch fundierte Hinweise dafür vor, dass sich Putnams Statement „Connectedness, not merely faith, is responsible for the beneficence of church people" (Putnam 2000: 67; 4.2.4) auch auf das Engagementverhalten in religiösen MSO in besonderem Maße zutrifft. Die Vielfältigkeit der Angebote macht Moscheevereine, in denen ganze Großfamilien eingebunden sind (Ceylan 2006: 79; Lemmen 2017: 315), zu viel besuchten Orten. Es scheint v. a. eine spezifische Qualität sozialer Interaktionsformen in der Gemeinde, die nicht allein durch die Frequentierung religiöser Veranstaltungen hergestellt wird, für die Mobilisierung von Engagement relevant zu sein (4.2.4; 4.2.5). Bei letzterer können wiederum kommunikationsstarke Amtsträger, wie Imame oder 
Priester, die Vertrauen und Respekt genießen, potenziell eine wichtige Rolle übernehmen (Baumann 2016: 85, 90 ff., 111; Schumacher/Huth 2013: 37; Halm et al. 2012a: 11, 454; Maloney et al. 2008: 272). Es dürfte schwerer fallen, Anfragen von mit Autorität oder Charisma ausgestatteten religiösen Amtsträgern hinsichtlich einer freiwilligen und unbezahlten Beteiligung in der Organisation abzulehnen. Darüber hinaus können Mechanismen der wechselseitigen sozialen Kontrolle relevant werden. Erwartungshaltungen und Aufforderungen von unmittelbar im Organisationskontext stehenden bekannten oder befreundeten Mitgliedern, sich einzubringen, können ebenfalls verhaltensregulierende Wirkung ausüben (Harris 2003: 122 f.; Kecskes/Wolf 1996: 25 f.; Wald et al. 2005: 132). Durch Inanspruchnahme der Leistungen, die eine Organisation anbietet, entsteht zudem ein Gefühl persönlicher Verpflichtung, sich durch eigene unbezahlte Leistungserbringung gegenüber der Gemeinschaft zu revanchieren (Klöckner 2016: 155, 432).

Zusammenfassend bleibt damit festzuhalten, dass Religion bzw. religiösen Vereinigungen im Kontext von Migration, Aufnahme- und Minderheitensituation in kulturell-sozialen Bereichen eine genuin eigenständige, alternativlose, aber auch kombinationsfähige und multifunktionale Rolle zu übernehmen und ein doppeltes Mobilisierungspotenzial zu besitzen scheinen ${ }^{74}$.

Diese Feststellung flankierend wird vor dem Hintergrund der in diesem Kapitel erfolgten Darlegungen davon ausgegangen, dass die Gewinnung von Engagierten in religiösen Organisationen einfacher ist und diese im Vergleich zu nicht-religiösen Vereinigungen daher einen Mobilisierungsvorteil besitzen.

Folgende Doppelhypothese wird formuliert:

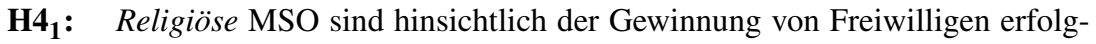
reicher als nicht-religiöse.

$\mathbf{H 4}_{2}$ : Familientraditionen, regelmäßige Veranstaltungen sowie die persönliche Ansprache von Freunden/Bekannten im religiösen Feld sind besonders wirksame Faktoren für die Rekrutierung von Engagierten in religiösen MSO.

\footnotetext{
${ }^{74}$ Diese auf empirischen Befunden basierende These konterkariert damit ein Stück weit die aufgrund theoretischer Annahmen in religiösen und nicht-religiösen Organisationen zu erwartenden Engagiertenmotive (4.3.3.2: $\mathrm{H}_{1}$ und $\mathrm{H}_{3}$ ). Insbesondere deshalb gilt es, die Beweggründe für freiwillige Engagements in institutionalisierten religiösen Kontexten zukünftig weiterhin zu erforschen.
} 


\subsection{Organisationales Handeln aus der Perspektive des soziologischen Neoinstitutionalismus}

Der soziologische Neoinstitutionalismus (im Folgenden kurz: NI) gehört in den USA und Europa zu den wichtigsten organisationstheoretischen Forschungsansätzen (Bonazzi 2014: 409). Er wurde in den 1970er Jahren von US-amerikanischen Organisationssoziologen begründet. ${ }^{75}$ In den letzten Jahrzehnten hat sich der NI auch in Deutschland zu einem populären Forschungsprogramm entwickelt ${ }^{76}$, dessen zentrale Konzepte auch für die Analyse und Einordnung des Handelns von MSO herangezogen werden (Rosenow-Williams 2012; Rosenow 2010; Pries/Sezgin 2010a; Nagel 2015b: 30 f.; Nagel/Plessentin 2015: 257 ff.). Die theoretisch-konzeptuellen Überlegungen der Protagonisten des NI fallen zum Teil verschiedenartig aus. ${ }^{77}$ Aufgrund der bestehenden Inkonsistenzen wird im Zusammenhang mit dem NI weniger von einer geschlossenen, einheitlichen Theorie, sondern vielmehr von einem „Forschungsprogramm“ gesprochen (Preisendörfer 2016: 173; Mense-Petermann 2006: 70 f.; Scott 1987).

Im Folgenden werden zwecks Einordnung und Orientierung in knapper Form zunächst wesentliche Charakteristika und Grundannahmen des NI skizziert. Im Anschluss erfolgt eine Erläuterung von Kernkategorien des NI in Verknüpfung mit zentralen Annahmen der Protagonisten des NI. Die Diskussion der theoretischen Prämissen bildet das Fundament für die Formulierung von insgesamt vier Hypothesen am Ende des Kapitels.

Innerhalb des NI gelten Organisationen - nach der dreiteiligen Organisationskonzeption von Scott und Davis - als offene Systeme (,open systems

\footnotetext{
${ }^{75} \mathrm{Zu}$ den drei für den NI richtungsweisenden Veröffentlichungen zählen die beiden 1977 erschienenen Aufsätze „Institutional Organizations: Formal Structure as Myth and Ceremony“ von John Meyer und Brian Rowan sowie „The Role of Institutionalization in Cultural Persistence“ von Lynne Zucker; im Jahr 1983 folgte der Beitrag „,The Iron Cage Revisited: Institutional Isomorphism and Collective Rationality in Organization Fields" von Paul DiMaggio und Walter Powell. Zur Profilierung des NI trug der von Walter W. Powell und Paul J. DiMaggio (1991) herausgegebene Sammelband The New Institutionalism in Organizational Analysis bei, der auch die drei genannten wichtigen Beiträge umfasst. Hinsichtlich letzterer wird in diesem Kapitel auf die im Sammelband von Powell und DiMaggio (1991) abgedruckten Versionen rekurriert.

${ }^{76}$ Faust 1992; Faust/Bahnmüller 1996; Vollmer 1996; Hasse/Krücken 1996, 2005; Türk 2000; Ortmann/Zimmer 2001; Bresser/Millonig 2003; Wilkens et al. 2003; Meyer 2004; Schimank 2007b; Walgenbach/Meyer 2008; Süß 2009; Senge 2005, 2006, 2011; Senge/Hellmann 2006a; Höllerer 2012.

$77 \mathrm{Zu}$ den Pionieren des NI zählen v. a. John Meyer, Brian Rowan, Paul DiMaggio, Walter Powell, Lynne Zucker und Richard Scott.
} 
view"), die im Austausch mit ihrer Umwelt stehen, von dieser maßgeblich beeinflusst werden und zu großen Teilen von ihr abhängig sind (Scott/Davis 2007: 112; Senge/Hellmann 2006b: 13). Neoinstitutionalisten nehmen keine handlungstheoretische und akteurzentrierte Analyseperspektive ein, sondern eine gesellschaftstheoretische, makrosoziologische. Eine Betrachtung von Organisationen als rational agierende Kollektivakteure lehnen sie ab. In den Analyseperspektiven und Erklärungsansätzen des NI spielt die „institutionelle“ Umwelt eine zentrale Rolle: Institutionen beeinflussen in Form von regulativen, normativen und kognitiven Einflüssen das Handeln von Organisationen, die damit nicht mehr ausschließlich als kontinuierlich zweckrational agierende Kollektivakteure, sondern vielmehr als Produkte ihrer gesellschaftlichen Umwelt(en ${ }^{78}$ betrachtet werden (Senge/Hellmann 2006b: 12; Rosenow-Williams 2012: 33; Rosenow 2010: 171). Gegenüber Rational-Choice-Ansätzen wird die soziale Bedingtheit des organisationalen Handelns stark gemacht (,,bringing society back in“). Im Gegensatz zu traditionellen organisationstheoretischen Ansätzen fokussiert der NI damit keine einzelnen Organisationen, sondern vielmehr den gesellschaftlichen Kontext in seinen Auswirkungen auf organisationales Handeln überhaupt (Bonazzi 2014: 410; Walgenbach 2014: 295 f., 299 f.).

Die neoinstitutionalistische Prämisse, dass das organisationale Handeln von spezifischen Regelkomplexen und Bedeutungssystemen beeinflusst wird (Scott 1994a: 203), gilt dabei sowohl für profitorientierte Unternehmen als auch freiwillige Vereinigungen. Der im ökonomischen Denken und in gewinnorientierten Unternehmen dominierenden Modellvorstellung des Homo oeconomicus, der nach der größtmöglichen wirtschaftlichen Nutzenmaximierung strebt und aufgrund einer vollständigen Informiertheit stets rational handeln wird, wird im NI das Leitmotiv des organisationalen Strebens nach Legitimität entgegengesetzt. Das oberste Ziel von Organisationen besteht darin, aus ihrer Umwelt die für ihren Bestand essenzielle Legitimität zu erhalten. Die damit einhergehenden Praktiken sind nicht in erster Linie nach technisch-ökonomischen Effizienzkriterien ausgerichtet, sondern können diesen sogar entgegenstehen (Preisendörfer 2016: 168). Hochbedeutsam ist damit das Verhältnis einer Organisation zu ihrer institutionellen Umwelt, auf deren wahrgenommenen Erwartungen die Organisationen auf unterschiedliche Weise reagieren. Die Art und Weise, wie die Umwelt von einer Organisation wahrgenommen und interpretiert wird, kann unterschiedlich - in

78 „Wegen der zunehmenden Ausdifferenzierung moderner Gesellschaften sprechen die Neoinstitutionalisten auch häufig von institutionellen Umwelten und nicht von institutioneller Umwelt (Scott 1987)“(Walgenbach 2014: 301; Herv. i. Orig.). 
einem Spektrum von positiv, über neutral bis negativ - ausfallen. So können einerseits Chancen, Optionen und Opportunitäten, andererseits aber auch Konkurrenz, Risiken und Bedrohungen ausgemacht werden.

Vor dem Hintergrund der herausgestellten Aspekte lässt sich der NI mit Paul J. DiMaggio und Walter W. Powell (1991a: 8) komprimiert charakterisieren als ,a rejection of rational-actor models, an interest in institutions as independent variables, a turn toward cognitive and cultural explanations, and an interest in properties of supraindividual units of analysis that cannot be reduced to aggregations or direct consequences of individuals' attributes or motives."

Im Folgenden werden die zentralen Termini und Konzepte in der Nomenklatur des NI in ihren Bezügen und in ihrer Relevanz für organisationale Performanzen erläutert. Angemerkt sei an dieser Stelle, dass innerhalb des NI zahlreiche konzeptuelle Spezifika, Varianzen sowie eine Fülle von Diskussionssträngen existieren, die in diesem Kapitel nicht alle thematisiert werden können. Zu klären sind die Fachbegriffe Legitimität, Isomorphie, Institution, organisationales Feld und Entkopplung. Ergänzt werden diese Schlüsselbegriffe - genauer: das Konzept des organisationalen Feldes - um das an anderer Stelle bereits thematisierte Konzept der Mitgliedschafts- und Einflusslogik (Schmitter/Streeck 1999/[1981]; 4.3.2; 4.3.3), das im NI bislang vernachlässigt wurde; ihm kommt innerhalb der Forschung zu nicht gewinnorientierten Organisationen, Verbänden und MSO aber ein wichtiger Stellenwert zu (Pries 2010a: 16, 27 ff.; Rosenow 2010: 171 f.; Rosenow-Williams 2012: 46 ff.).

Wie bereits erwähnt, wird vom NI die Handlungslogik des einzelnen Akteurs innerhalb einer Organisation ausgeblendet; stattdessen ist das Zustandekommen von Legitimität gegenüber unterschiedlichen Erwartungsgruppen in der Umwelt der Organisation die vorrangige Aufgabe - und zwar unabhängig von technischökonomischen Effizienzkriterien. Marc Suchman (1995: 574; Herv. i. Orig.) hat unter Berücksichtigung zahlreicher Definitionen von Legitimität eine Begriffsbestimmung vorgenommen, auf die im NI weithin rekurriert wird: „Legitimacy is a generalized perception or assumption that the actions of an entity are desirable, proper, or appropriate within some socially constructed system of norms, values, beliefs, and definitions." Nur wenn Legitimität gewonnen und aufrechterhalten werden kann, kann die Organisation auf Anerkennung, Wertschätzung und Unterstützung, z. B. in Form von personeller oder finanzieller Ressourcenzufuhr, bauen, und damit ihre Überlebens- und Leistungsfähigkeit, Stabilität und ihren Erfolg sichern (Hellmann 2006: 75; Meyer/Rowan 1991: 53).

Die beiden Grundannahmen, dass die gesellschaftliche, institutionelle Umwelt das Handeln von Organisationen wesentlich beeinflusst und diese nach Legitimität streben, resultieren den frühen Neoinstitutionalisten zufolge in Prozessen der 
Isomorphie $^{79}$ - d. h. der Anpassung, Angleichung oder Adaption - in Bezug auf Arbeitsweisen, Zielsetzungen, Strukturen und die öffentliche Selbstpräsentation von Organisationen. Dabei fungieren andere Organisationen in einem bestimmten Bereich der Umwelt als Vorbilder, sofern sie als besonders legitim und erfolgreich wahrgenommen werden. Laut DiMaggio und Powell (1991b: $67 \mathrm{ff}$.) führen drei Mechanismen zu den organisationalen Angleichungsbestrebungen: 1.) Isomorphie durch Imitation/Nachahmung (,mimetic isomorphism“), 2.) Isomorphie durch Zwang (,coercive isomorphism“) und 3.) Isomorphie durch normativen Druck (,normative isomorphism“) (s. auch Hasse/Krücken 2005: 25 ff.).

Isomorphie durch 1.) mimetische Prozesse ist auf gegenseitige Beobachtungen von Organisationen aufgrund von Unsicherheit zurückzuführen. Organisationen, die als besonders legitim und erfolgreich eingeschätzt werden, weil deren Praktiken und Strukturen als besonders zweckdienlich erscheinen, werden als Vorbilder imitiert bzw. kopiert. Zudem werden Einstellungen und Erwartungen anderer Feldakteure antizipiert (Pries 2010a: 38). Isomorphie erfolgt 2.) durch Zwang, und zwar aufgrund der notwendigen Einhaltung von gesetzlichen Vorgaben, durch Anweisungen oder wegen Abhängigkeitsverhältnissen zu anderen, z. B. staatlichen oder politischen, Organisationen. Anpassung durch 3.) normativen Druck kann sich aus Professionalisierungsanforderungen ergeben, die von außen an die Organisationen herangetragen werden, wie etwa in Bezug auf die Festlegung von Richtlinien oder auf Entwicklung und Einhaltung professioneller Standards; zudem können kulturelle Erwartungen der Gesellschaft sowie geteilte Leitbilder und Vorstellungen normativen Druck ausüben (DiMaggio/Powell 1991b: 70 f.; Becker-Ritterspach/Becker-Ritterspach 2006a: 110).

Im frühen neoinstitutionalistischen Denken wird davon ausgegangen, dass es im Rahmen des Prozesses der Isomorphie zu einer Strukturangleichung kommt, indem Organisationen in ihrer gesellschaftlichen Umwelt vertretene Werte, Regeln und kulturelle Praktiken als strukturelle Elemente in ihre eigene interne Struktur integrieren (Meyer/Rowan 1991; Walgenbach 2014: 295 f.). Bei den aus der institutionellen Umwelt übernommenen strukturellen Elementen kann es sich z. B. um bestimmte Verfahrensweisen, Arbeits- und Managementtechniken, Programme oder Stellenprofile handeln, die im Zusammenhang mit der nicht auf ökonomische Effizienz ausgerichteten Erfüllung der Organisationsaufgaben stehen. Als ,prefabricated formulas“ (Meyer/Rowan 1991: 44) und „packaged codes“ (ebd.: 45) sind die übernommenen Strukturelemente institutionalisiert und rationalisiert, weil

\footnotetext{
${ }^{79}$ Isomorphie: von gleicher Gestalt, Form; aus dem Griechischen, von isos = gleich und morphe $=$ Gestalt, Form.
} 
an ihre Vernunftgemäßheit geglaubt wird, und sie sich damit legitimitätsstiftend auswirken.

Die drei für den Formangleichungsprozess genannten relevanten DruckMechanismen - Imitation, Zwang, normativer Druck - können sich durchaus überlappen und zusammen wirken (Walgenbach 2014: 314 f.). Sie korrespondieren mit den im NI und für das organisationale Handeln als unabhängige Variablen fungierenden Institutionen, die das Verhalten von Individuen und Kollektiven in der Gesellschaft anleiten. Institutionen umfassen normative Vorgaben, informelle und formelle Regeln, kulturelle Standards und eingespielte Praktiken (Preisendörfer 2016: 168; Walgenbach 2014: 299). Konstanze Senge definiert Institutionen - in Anlehnung an die von Niklas Luhmann vorgenommene Einteilung - in eine zeitliche, soziale und sachliche Sinndimension als soziale Regeln mit handlungsregulierender Kraft, ,die das organisationale Geschehen in zeitlicher Perspektive dauerhaft (sie gelten lange), in sozialer Hinsicht verbindlich (Akteure halten sich daran) und in sachlicher Hinsicht maßgeblich (die Institution ist für ein Phänomen bedeutsam) beeinflussen" (Senge 2006: 35, Herv. i. Orig.; s. ebd., Fn 1).

Im NI wird vielfach auf das von Richard Scott in seinem Buch Institutions and Organizations (2014) dargelegte Drei-Säulen-Modell von Institutionen rekurriert. Scott (2014: 56 ff.) unterscheidet zwischen regulativen, normativen und kulturell-kognitiven Dimensionen von Institutionen, die er - den beschriebenen Isomorphie auslösenden Mechanismen inhaltlich entsprechend - handlungsregelnde Kräfte zuweist. Mit dem Drei-Säulen-Modell werden unterschiedliche Arten bzw. tragende Elemente von Institutionen charakterisiert. Die drei Säulen konstituieren oder unterstützen Institutionen und verleihen dem sozialen Verhalten Bedeutung und Stabilität (Senge 2006: 39; Scott 2014: 59 ff.).

Die regulative Dimension von Institutionen entfaltet ihre Wirkung qua Gesetz und Regeln - durch Zwang. Akteure befolgen Regeln und Gesetze, um negative Sanktionen zu vermeiden und positive Sanktionen zu erhalten (Scott 2014: 59 ff.). Die normativ gerahmten Institutionen induzieren Verhaltensweisen, die auf Normen und Werten basieren, die sozial erwünschte Umgangsformen vorschreiben, die also vorgeben, wie man sich (nicht) verhalten soll. Scott zufolge hängt die Handlungswirksamkeit dieser Normen zum einen vom Grad ihrer subjektiven Internalisierung $a b, d$. h. inwiefern sie sich zu eigen gemacht wurden und sie als kontrollierende Instanzen fungieren. Zum anderen spielt auch der Erwartungsdruck anderer Akteure, sich normenkonform zu benehmen, eine Rolle (ebd.: 64 ff.). Kulturell-kognitive Institutionen beziehen sich auf die kulturell determinierte Wahrnehmung und Erfahrung der Realität, die auf geteilten Vorstellungen basieren, die die Natur der sozialen Realität und die bedeutungsgenerierenden 
Rahmen konstituieren (ebd.: 66 ff.). Dazu zählen insbesondere „,common meaning systems“, „wider belief systems and cultural frames“ oder ,common framework[s] of meanings“ (ebd., 48, 69, 70; vgl. auch Berger/Luckmann 2012/[1969]). Zentral ist die Auffassung der kulturell-kognitiven Institutionen als Skripte, d. h. als Verhaltensabläufe, die wie selbstverständlich und automatisch ablaufen und die Routine reproduzierende Verfahren darstellen: Institutionen werden durch „quasi-automatische Verhaltensabläufe (Skripte) unterstützt und aufrechterhalten“ (Walgenbach 2014: 298; s. auch 4.3.3.2). Die Skripte sind als kognitive Strukturen gesellschaftlich verankert und beeinflussen das Handeln von Akteuren in Form von Wenn-Dann-Regeln: „Compliance occurs in many circumstances because other types of behavior are inconceivable; routines are followed because they are taken for granted as "the way we do these things" "(Scott 2014: 68). Die kulturellkognitive Dimension der Institutionen stellt für Scott das wichtigste soziologische Distinktionskriterium des NI dar (ebd.: 47, 67). Auch DiMaggio und Powell (1991a: 15) konstatieren: „Not norms and values but taken-for-granted scripts, rules, classifications are the stuff of which institutions are made" .80

Routinemäßig ablaufende Verhaltensmuster werden im NI daher weniger unter der Perspektive einer Kosten-Nutzen-Kalkulation betrachtet. Dies bedeutet aber keinesfalls, dass Neoinstitutionalisten dem Prinzip der Rationalität eine vollständige Absage erteilen. So sind Entscheidungen in Organisationen durchaus rational intendiert. Neoinstitutionalisten gehen aber davon aus, dass die institutionellen Einflüsse unreflektierte Entscheidungen befördern (Senge/Hellmann 2006b: 17), die nicht auf dem ökonomischen Effizienzdenken einer Zweck-MittelRelation basieren. Der Rationalitätsgrad von Zweck-Mittel-Konnexen vermittelt sich vielmehr über Mythen, an deren Zweckmäßigkeit geglaubt wird bzw. die nur vorgeben, vernunftgemäß und sinnvoll zu sein (Meyer/Rowan 1991; Meyer 1992,

\footnotetext{
${ }^{80}$ Als theoretischer Bezugspunkt der beiden Begriffe „Institution“ und „Institutionalisierung“ im NI fungiert u. a. der wissenssoziologische Ansatz von Peter Berger und Thomas Luckmann (2012/[1969]), die von der sozialen Konstruktion bzw. subjektiven Gewissheit von Wirklichkeit durch alltagsweltliche Erfahrungen der Individuen ausgehen. Die Subjekte streben in Bezug auf das eigene und das (zukünftige) Handeln von anderen Personen nach Erwartungssicherheit, die ihnen in Form von intersubjektiv geltenden Institutionen bereitgestellt wird. Die Institutionalisierungsdynamik ergibt sich aufgrund von Habitualisierungen und Routinisierungen, die das Individuum von Reflexions- und Entscheidungszwängen entlasten, sowie aufgrund von Typisierungen von Handlungsweisen (Berger/Luckmann 2012/[1969]: 56 ff.). „Alles menschliche Tun ist dem Gesetz der Gewöhnung unterworfen. Jede Handlung, die man häufig wiederholt, verfestigt sich zu einem Modell, welches unter Einsparung von Kraft reproduziert werden kann und dabei vom Handelnden als Modell aufgefaßt wird. Habitualisierung in diesem Sinne bedeutet, daß die betreffende Handlung auch in Zukunft ebenso und mit eben der Einsparung von Kraft ausgeführt werden kann“ (ebd.: 56, Herv. i. Orig.).
} 
1994). ${ }^{81}$ Organisationen handeln Meyer und Rowen zufolge auf Basis ,,rationalisierter Mythen“ sowie einer „Logik des Vertrauens und des guten Glaubens“ (Meyer/Rowan 1991: 58 ff.). Rationalität bzw. rationales Handeln erscheinen somit dominant in Form eines Mythos: „im Sinne einer Wahrheit des Glaubens“ (Tacke 2006: 96; Herv. i. Orig.). Aufgrund des Mechanismus der Institutionalisierung muss der Eintritt erwarteter, als selbstverständlich geltender kausaler Effekte nicht überprüft werden (ebd.; Becker-Ritterspach/Becker-Ritterspach 2006a: 104 f.). Individuen in Organisationen handeln damit auf Grundlage kognitiver Skripte routiniert-institutionalisiert und nicht in erster Linie als exakt rational kalkulierende Akteure. Die hartnäckige gesellschaftliche Erwartung von Rationalität - von der Ratio geleitetem Handeln - ,beantwortet der NI mit der Institutionalisierung von Regeln, die diese Erwartung enthalten und aufrechterhalten“ (Tacke 2006: 93). Somit nehmen unreflektierte, unbewusst ablaufende Entscheidungsabläufe bzw. routinierte, selbstverständliche Handlungsformen von Organisationen eine Schlüsselfunktion ein.

Die drei Säulen des Scottschen Modells gewährleisten allesamt den Fortbestand der Institutionen. Seine Konzeption umfasst unterschiedliche Kontinua zwischen als selbstverständlich (,taken-for-granted“) betrachteten oder gesetzlich sanktionierten Verhaltensweisen einerseits und bewusst oder unbewusst (re-)produzierten Praktiken und Strukturen andererseits (Walgenbach 2014: 319).

Die erläuterten institutionellen Einflüsse scheinen organisationale Strukturen und Praktiken zu formen, wodurch die Organisationen gleichsam als Produkte ihrer Umwelt erscheinen (Meyer/Jepperson 2000). Dies wurde auch bereits mit dem zentralen Begriff der legitimitätsgenerierenden Isomorphie thematisiert, auf den im Weiteren allerdings noch differenzierter einzugehen ist.

Mit der Einführung des Begriffs des organisationalen Feldes durch DiMaggio und Powell (1991b) wurde der Versuch unternommen, im NI eine zentrale Analyseeinheit festzulegen (Senge 2005: 95; Becker-Ritterspach/Becker-Ritterspach 2006b: 118). DiMaggio und Powell (1991b) definieren als organisationales Feld das räumlich eingrenzbare Gebiet, innerhalb dessen Prozesse der Angleichung und des Sich-ähnlich-Werdens von Organisationen entsprechend institutioneller

\footnotetext{
${ }^{81}$ In komplexen, ausdifferenzierten Gesellschaften können Vorstellungen darüber, welche Mittel mit Blick auf die Erreichung angestrebter und wünschenswerter Zwecke als rational gelten (können), in unterschiedlichen sozialen Zusammenhängen und Anspruchsgruppen generell weit auseinandergehen. Auch die Definition des Begriffs der Kognition variiert im NI aufgrund verschiedener theoretischer Verortungen (s. hierzu Klatetzki 2006: $55 \mathrm{ff}$.). Zudem ist die neoinstitutionalistische Auffassung von Kognitionen als überindividuelle Makro-Entitäten zu problematisieren und die resultierende Diagnose des „Aufwärtsreduktionismus“ des NI zu beachten (ebd.: $58 \mathrm{ff}$.).
} 
Umweltbedingungen stattfinden. Bezogen auf gewinnorientierte, marktförmige Organisationen umfasst ein organisationales Feld nach DiMaggio und Powell all diejenigen „Organisationen, die insgesamt einen anerkannten Bereich des institutionellen Lebens konstituieren: wichtige Lieferanten, Ressourcen- und Produktkonsumenten, Aufsichtsbehörden und andere Organisationen, die ähnliche Produktdienstleistungen erbringen“ (DiMaggio/Powell 1991b: 64 f.; Übers.: d. Verf.). Dieser Definition sind ergänzend die Kategorisierungen von Scott beiseitezustellen, der das organisationale Feld - in Anlehnung an DiMaggio und Powell (1991b) - als wichtige intermediäre Einheit betrachtet, über die organisationale Strukturen und Arbeitsweisen mit Prozessen auf der gesellschaftlichen Ebene verknüpft werden. Das organisationale Feld umfasst nach Scott eine Gemeinschaft von interdependenten Organisationen, die häufig und schicksalhaft innerhalb gemeinsamer Regeln, Normen und Bedeutungssysteme miteinander interagieren (Scott 2003: 130; Scott 1994a: 207 f.).

Hinsichtlich der Strukturierung und Institutionalisierung des organisationalen Feldes bestimmen DiMaggio und Powell (1991b: 65) vier analytische Charakteristika: 1.) eine Zunahme von Interaktionen zwischen Organisationen im Feld; 2.) die Entstehung von hierarchischen Beziehungen und Koalitionen zwischen Organisationen; 3.) eine Zunahme der mit Erwartungen verknüpften zu bewältigenden Informationslast; 4.) das gegenseitige, erstarkende Bewusstsein der Organisationen, dass sie in eine dynamische institutionelle Umwelt eingebunden und mit ähnlichen Herausforderungen konfrontiert sind. Die zentrale Annahme ist, dass Organisationen innerhalb eines organisationalen Feldes miteinander interagieren, dass sie sich hinsichtlich ihrer Handlungen und Strategien aneinander orientieren und sich dadurch immer ähnlicher werden. Da die institutionelle Umwelt einen hohen Organisationsgrad aufweist, führen die gegenseitigen Bezugnahmen und Reaktionen der Organisationen untereinander zu einer Homogenisierung des organisationalen Feldes insgesamt (DiMaggio/Powell 1991b; ähnlich Scott 1992; Zucker 1983).

Mit der Weiterentwicklung des NI wurden die Kernannahmen zur Isomorphie zunehmend - aber nicht grundsätzlich - infrage gestellt. Angezweifelt wurde die Vorstellung einer quasi wie von selbst erfolgenden Struktur- und Formangleichung. Verwiesen wurde vielmehr auf eine erforderliche Berücksichtigung von mehreren, unterschiedlichen organisationalen Feldern und Analyseebenen, wie etwa einem transnationalen Kontext, in dem Organisationen agieren. Es sei von heterogenen und nicht von gleichförmigen Anpassungsprozessen auszugehen, weniger von Isomorphie, als vielmehr von Polymorphie, d. h. eher von der Ausbildung hybrider neuer Organisationsformen, die keineswegs identisch sind (Becker-Ritterspach/Becker-Ritterspach 2006a: 111 ff.). Eine umfassende 
Homogenisierung aufgrund einer getreuen Imitation sei nicht zu erwarten, da infolge unvollständiger Imitationen vielmehr mit Innovationen zu rechnen sei (ebd.: 112). Dies haben auch bereits DiMaggio und Powell (1991b: 69) herausgestellt. Auch sei während des Imitationsprozesses mit „Übertragungsfehlern“ (Quack 2006: 175) zu rechnen und generell umfasse jeder Imitationsversuch zwangsläufig innovative Elemente (ebd.; s. auch Zucker 1977). Für die Revision des Isomorphie-Konzepts waren auch die theoretischen Überlegungen um das Translationskonzept im Skandinavischen Institutionalismus (SI) bedeutsam. Dessen Grundannahme ist, dass die Nachahmung von organisationalen Formen und Praktiken innerhalb anderer, nicht deckungsgleicher Kontexte deren Übersetzung (Translation) notwendig macht, was immer Veränderungen und die Entstehung von neuen Formen und Praktiken impliziere und damit eine vollständige Isomorphie ausschließe (Becker-Ritterspach/Becker-Ritterspach 2006a: 113). ${ }^{82}$

Angesichts der dargelegten Annahmen ist hinsichtlich des Forschungsgegenstandes dieser Arbeit - den MSO - nachfolgend auf bislang unerwähnte, aber einflussreiche Kontextbedingungen und durch diese potenziell ausgelöste Reaktionen der migrantischen Vereinigungen einzugehen.

Den Begriff des organisationales Feldes wendet Ludger Pries (2010a: 24) dezidiert auf MSO an: Er versteht darunter die „Gesamtheit aller (anderen) Organisationen [...], die für eine bestimmte Organisation als Bezugseinheiten und Legitimationsadressaten ihres kollektiven Handelns bedeutsam sind (für Migrantenorganisationen z. B. andere Migrantenorganisationen, Gewerkschaftsverbände, politische Parteien, staatliche Verwaltungseinheiten, Ausländerbeiräte etc.).“ Wie oben dargelegt wurde, ist für die Konstituierung eines organisationalen Feldes Voraussetzung, dass die Organisationen miteinander interagieren und sich dieser

\footnotetext{
${ }^{82}$ Mit Florian und Jutta Becker-Ritterspach (2006a: 116) bleibt somit festzuhalten, dass institutionelle und isomorphe Kräfte im NI weiterhin relevant sind; „Begriffe wie Transformation, Translation oder Hybridisierung [...], die auf Veränderung und Ungleichheit verweisen“, sowie „Überlegungen [zu] unvollkommener Institutionalisierung und Polymorphie“ treten jedoch zunehmend an die Stelle der Isomorphie. Diese Umorientierung sei vier Aspekten geschuldet: und zwar 1.) der „Problematisierung des Feldbegriffs“; 2.) der „Einführung von Translations- und Hybridisierungsansätzen“; 3.) der „Berücksichtigung von Organisationen als Akteure und von Akteuren in Organisationen“ sowie 4.) ,Überlegungen zu Entwicklungen der Transnationalisierung“ (ebd.).
} 
Interaktionen bewusst sind ${ }^{83}$ Wichtige Faktoren, die die Interaktionen und Handlungen der MSO innerhalb des organisationalen Felds wesentlich mitbestimmen, betreffen das Ausmaß von Wettbewerb und Konkurrenz sowie die dynamischen Beziehungen zu Akteuren im Ausland (Hunger/Metzger 2011, 2013a; Olgun 2015; Blätte 2014; Halm et al. 2012a; Rosenow-Williams 2012). Dabei geht es um einen Wettstreit um finanzielle Ressourcen, Mitglieder, Zielgruppen, politische Legitimität, die Unterstützung durch die Mitglieder sowie die Öffentlichkeit wie auch um einen allgemeinen Wettbewerb sowohl zwischen den islamischen Verbänden als auch zwischen religiösen und nicht-religiösen MSO im Allgemeinen (Rosenow-Williams 2012: 231, 437 ff., 469).

Vor diesem Hintergrund agieren MSO in einem Spannungsverhältnis von Erwartungen und Ansprüchen der eigenen Gruppe einerseits sowie verschiedenen Akteuren der Aufnahmegesellschaft andererseits (Pries 2010a: 39; 4.3.5). Es bestehen Wechselwirkungen zwischen Binnen- und Außenwelt, wobei das Handeln und Auftreten der MSO ganz wesentlich mitbestimmt werden von der Art der Wahrnehmung öffentlicher Diskurse und von ihrer Behandlung durch die gesellschaftliche Umwelt (Pries 2013a: 5, 2010a: 20; Nagel 2015b: 31). Somit bleiben auch öffentliche und medial vermittelte Debatten nicht ohne Folgen und sind als wichtige Umweltfaktoren mitzudenken (3.2). Abwertende Meinungen oder Anfeindungen können sowohl lähmende als auch aktivierende Effekte auf die zivilgesellschaftlichen Aktivitäten und das Kooperationsverhalten haben, wobei keine eindeutigen Reaktionen und Folgen auszumachen oder vorherzusagen sind (Nagel/Plessentin 2015: 264).

In diesem Kontext wäre zu hinterfragen, ob Wahrnehmung von Fremdenfeindlichkeit mit allgemeinen Rückzugstendenzen einhergeht, da gerade Kooperationen mit anderen Organisationen, von denen keine Diskriminierungen ausgehen, diesen negativen Erfahrungen entgegenwirken sollten. Jedoch können solche proaktiven Reaktionen nicht automatisch angenommen werden. Denn die Erfahrung von Ausgrenzung und mangelnder Akzeptanz kann begleitet sein von der Wahrnehmung geringer Teilhabechancen und in Rückzug resultieren. Unmut und Frustration über Negativ-Diskurse und die seitens der Mehrheitsgesellschaft verwehrte Zugehörigkeit können dazu führen, dass Entwicklungen positiver Einstellungen gegenüber

\footnotetext{
${ }^{83}$ Für empirische Studien stellt sich die Herausforderung der Identifizierung des organisationalen Feldes einer Fokalorganisation in Bezug auf die Berücksichtigung aller relevanten Organisationen in ihren diversen Beziehungen, die sich alle wiederum äußerst dynamisch entwickeln können. Zu den Schwächen des Feldbegriffs als Analyseeinheit des NI wird daher auch die Schwierigkeit seiner ,Abgrenzbarkeit und empirische[n] Handhabbarkeit“ (BeckerRitterspach/Becker-Ritterspach 2006b: 133) gezählt, was letztlich auch die Frage nach seiner empirischen Tragfähigkeit aufwirft.
} 
der Mehrheitskultur ausbleiben und eine stärkere Selbstsegregation forciert wird (Heitmeyer et al. 1997; Toprak/Weitzel 2019). Alternativ ermöglicht die Konzentration auf die eigene Gruppe den Gewinn von Status, Anerkennung und das Gefühl von Zugehörigkeit und Gemeinschaft (Uslucan 2016).

Vor diesem Hintergrund zeigen Fallstudien, dass Negativ-Diskurse aufseiten der Betroffenen nicht zwangsläufig zu Resignation und Abgrenzungen führen, sondern sie auch ,,als Herausforderung zu Aufklärung und Positionierung empfunden werden“ (Nagel/Plessentin 2015: 264) und Vernetzungen sowie Interessenvertretung begünstigen können. Neben Rückzügen sind demnach auch offensive Vorstöße möglich (3.2). Diese Reaktionen implizieren, dass es neben der im NI akzentuierten „Anpassung“ auch weitere Reaktionsformen gibt, die nachfolgend ebenfalls noch näher zu beleuchten sind.

Deutlich geworden ist, dass das Handeln von Organisationen im NI untrennbar mit der gesellschaftlichen Umwelt verknüpft ist. Eine wesentliche Herausforderung von Organisationen besteht somit darin, dass sie mit vielschichtigen, heterogenen Erwartungshaltungen konfrontiert werden können, die sich unter Umständen widersprechen und miteinander kollidieren: „Entscheidend ist [...], daß Organisationen einer Vielzahl konfligierender institutionell verankerter gesellschaftlicher Kontexte ausgesetzt sind, die in kausaler Beziehung zu den Prozessen und Entscheidungen in Organisationen stehen“ (Senge/Hellmann 2006b: 19). Entsprechend ist eine Legitimation gegenüber diversen Anspruchs- und Erwartungsgruppen vonnöten. So müssen organisationsexterne Ansprüche auch mit den organisationsinternen Erwartungen der Mitglieder in Einklang gebracht und gegenüber diesen muss ebenfalls Legitimität erlangt werden. Diese Prämisse ist an die für die Organisationsforschung grundlegenden Termini der Mitgliedschaftsund der Einflusslogik geknüpft, die von Philippe C. Schmitter und Wolfgang Streeck in ihrem vielzitierten Aufsatz „Die Organisation von Geschäftsinteressen“ (Schmitter/Streeck 1999/[1981]) konturiert wurden. Im NI wurde sie bislang vernachlässigt, worauf in den letzten zehn Jahren aber aufmerksam gemacht wurde (Pries 2010a: 16, 27 ff.; Rosenow 2010: 171 f.; Rosenow-Williams 2012: $46 \mathrm{ff}$.). Schmitter und Streeck reklamieren eine doppelte Organisationsverantwortung, die darin besteht, im Interesse der Sicherstellung von Ressourcenzufuhr (Subventionen, Anerkennung etc.) die Logik der Mitgliedschaft mit der Logik der Einflussnahme auszubalancieren (4.3.2; 4.3.3). Denn zivilgesellschaftliche Organisationen wie MSO sind in ihrer Existenzfähigkeit auf Mitglieder als ihre fundamentale Ressource angewiesen, die an die Organisation gebunden und deren Interessen folglich berücksichtigt werden müssen. Die Legitimität und Verfügbarkeit von internen und externen Ressourcen hängen von den Mitgliedern der 
Organisation und deren Logik(en) ab. Darüber hinaus bedarf es aber der Einflussnahme, z. B. auf öffentliche Behörden, um Anerkennung, Status und finanzielle Unterstützung zu erhalten. Die mit adäquaten organisationalen Handlungsweisen zu erlangende Legitimität - in Form der externen Anerkennung als integrer und vertrauenswürdiger Akteur - kann in Widerspruch zu Positionen der Mitglieder stehen, was die Organisation in die Rolle eines Vermittlers drängt. Für die Herstellung von Legitimität gegenüber widersprüchlichen Erwartungshaltungen heterogener Anspruchsgruppen, mit denen es angemessen umzugehen gilt, besitzt die Strategie der Entkopplung im NI eine besondere Bedeutung. Sie wird als ein probates Mittel zur Entflechtung des binnen- und außenorientierten organisationalen Handelns betrachtet, das zur Legitimitätserlangung nach innen wie nach außen beiträgt.

„Entkopplung“ wirft unter Neoinstitutionalisten zugleich die Frage auf, inwieweit die Angleichung von Strukturen und Praktiken an die Umwelt - die Isomorphie - durch Organisationen tatsächlich realisiert oder ob sie lediglich öffentlich kundgetan und vorgetäuscht und damit, zur Legitimitätssicherung, die Konformität mit gesellschaftspolitischen Anforderungen nur suggeriert wird. Während DiMaggio und Powell (1991b) von einer de facto vollzogenen Strukturangleichung ausgehen, vertreten Meyer und Rowan (1991) die Auffassung von lediglich in der Öffentlichkeit strategisch vollzogenen Verlautbarungen. Nach Meyer und Rowan ermöglicht die Entkopplung den Organisationen ,to maintain standardized, legitimating, formal structures while their activities vary in response to practical considerations“ (Meyer/Rowan 1991: 58). Dieses Verständnis impliziert zwei mit der Entkopplung einhergehende Aspekte, nämlich zum einen die Separierung von formalen Strukturen und Praktiken sowie zum anderen die Möglichkeit der Abkopplung von Strukturelementen untereinander, z. B. durch die Einrichtung von neuen Abteilungen (ebd.: 57). Damit sind die Formalund die Aktivitätsstruktur von Organisationen nach Meyer und Rowan nur lose miteinander gekoppelt (,loosely coupled“, ebd.: 60) und eine gesellschaftliche Anpassung findet nur demonstrativ über die Errichtung einer ,zeremonielle[n] Fassade“ (ebd.: 59) statt, um den Schein der Legitimität der Organisation zu wahren. Die in der Öffentlichkeit präsentierte Legitimitätsfassade kann sich erheblich von alltäglichen organisationsinternen Praktiken unterscheiden. So kann es sich bei der Betonung der Übernahme von gesellschaftspolitischen Verantwortlichkeiten, wie etwa dem Umweltschutz, der Frauenförderung oder der Beherzigung einer Gleichbehandlung der Geschlechter, lediglich um Lippenbekenntnisse und 
Mythen handeln. ${ }^{84}$ Der Schein kann so gewahrt und die Unterstützung sowohl durch den Kreis der Mitglieder als auch seitens externer Akteure sichergestellt werden (Preisendörfer 2016: 168 f.; Walgenbach 2014: 317 f.). In diesem Zusammenhang ist für die Erhaltung der Legitimationsfassade auch die Nutzung einer bestimmten passenden Sprache bzw. eines bestimmten Vokabulars von Bedeutung: Meyer und Rowan sprechen von „legitimated vocabularies“ (Meyer/Rowan 1991: 50), die nicht den tatsächlichen operativen Kernaktivitäten entsprechen müssen, was Nils Brunsson (1989) in der terminologischen Differenzierung zwischen „talk“ und ,action“ pointiert: der symbolischen sprachlichen Präsentation der Organisation einerseits und dem tatsächlichen Handeln andererseits. Brunsson fasst dieses Verhalten zum Zwecke des Umgangs mit verschiedenen Umweltanforderungen als organisationale Strategie der „Heuchelei“ auf (Brunsson 1989: 27).

Wie oben anhand der Beispiele zu möglichen Reaktionen auf öffentliche Diskurse seitens der MSO bereits deutlich wurde, stellen die Mechanismen der Anpassung (Isomorphie) und Entkopplung nicht die einzigen Handlungsoptionen für Organisationen dar, um auf unterschiedliche Erwartungen zu reagieren bzw. letztere miteinander in Einklang zu bringen. Die deterministischen Annahmen eines passiven, gleichförmigen, lediglich reaktiven und Konformität erzeugenden organisationalen Handelns - eines aus Fremdsteuerung resultierenden Adaptionsautomatismus - wurden auch von einigen Protagonisten des NI kritisiert. Andere offensive Reaktionsstrategien wie Widerstand, Intervention oder Manipulation würden komplett ausgeblendet. Zudem blieben organisationale Eigeninteressen, strategische Taktiken und Machtverhältnisse unberücksichtigt; in Druck ausübenden institutionellen Kontexten sei jedoch durchaus variationsfähiges organisationales Handeln zu beobachten (Perrow 1985, 1986; Powell 1985, 1991; DiMaggio 1988, 1991; Zucker 1987; Covaleski/Dirsmith 1988; Reed 1992; Aldrich 1992; Scott 1994b; Beckert 1999). Nicht zuletzt wurde die fehlende mikrosoziologische Fundierung als Defizit moniert. Die Organisationen erschienen als eine Black Box, indem „die alltäglichen internen Prozesse im Dunkeln blieben“ (Mense-Petermann 2006: 72) und lediglich die Reaktionen und Antworten von

${ }^{84}$ Die Begriffe „Lippenbekenntnisse“ und „Mythen“ dürften Assoziationen mit Effekten sozial erwünschten Antwortverhaltens in empirischen Befragungen hervorrufen. Die soziale Erwünschtheit betrifft Tendenzen bei der befragten Person, die Wahl von Antworten entsprechend angenommener sozialer Erwartungen und Normen vorzunehmen. Damit einher geht die Mitteilung von positiven Eigenschaften und Selbstdarstellungen (Häder 2010: 209 ff.). Die Qualität solcher Antworten kann als vermindert gelten, da hier eine Vortäuschung bzw. Verstellung stattfindet. 
Organisationen als Kollektive auf institutionelle Einflüsse, nicht aber diejenigen einzelner Akteure untersucht würden. Dem neoinstitutionalistischen Modell wurde damit häufig eine „Übersozialisierung“ des Verhaltens von Organisationen und deren Mitgliedern attribuiert (Granovetter 1985; Ortmann et al. 1997).

In Bezug auf die als notwendig erachtete Anerkenntnis eines Variationspotenzials im Organisationshandeln schlägt Christine Oliver vor, zur Erfassung aller möglichen organisationalen Reaktionsweisen auf institutionelle Umweltbedingungen eine integrierte Perspektive einzunehmen. Als konzeptionelle Grundlage greift Oliver hierfür auf konvergierende Grundannahmen des NI sowie des Ressourcenabhängigkeitsansatzes (engl. ,resource dependence theory“, kurz: RDT; 4.3.2) zurück $^{85}$. In ihrem populären Aufsatz „Strategic Responses to Institutional Processes“ (1991) entwickelt Oliver eine Typologie mit fünf strategischen organisationalen Reaktionen, die - in Abhängigkeit von der Art und dem Kontext der institutionellen Bedingungen - von passiver Konformität bis zu proaktiver Manipulation reichen. Zu den strategischen Handlungsoptionen zählen Duldung, Kompromiss, Vermeidung, Trotz und Manipulation (Oliver 1991: 152 ff.), wobei mit diesen wiederum jeweils ausdifferenzierte Taktiken verbunden sind (Tab. 4.4). Die Strategien und Taktiken umfassen - in aufsteigender Reihenfolge von organisationaler Passivität bis zu zunehmendem aktiven Widerstand - ein Repertoire an Verhaltensweisen, das Organisationen als Reaktion auf institutionelle Erwartungen und Pressionen in Abhängigkeit von ihrer Bereitschaft und ihrer Fähigkeit, sich dem Umfeld anzupassen, zeigen können (ebd.: 159).

Die Duldung korrespondiert mit der im NI stark betonten Isomorphie bzw. Anpassung oder Adaption. Der Kompromiss impliziert einen in der Regel aus Konflikten resultierenden Verhandlungsprozess, während sich die Vermeidungsstrategie auf die Verschleierung von Nichtkonformität mit institutionellen

\footnotetext{
${ }^{85}$ Sowohl Vertreter des NI als auch der RDT gehen davon aus, dass organisationale Entscheidungen im Kontext externer Beschränkungen möglich sind. Allerdings unterstreichen Neoinstitutionalisten in Bezug auf die Erlangung von Organisationsstabilität die Bedeutung von Konformität anstelle von Widerstand, von Passivität gegenüber Aktivität und von Akzeptanz anstatt Manipulation als Reaktionen auf externe Erwartungen und Zwänge (Oliver 1991: 149). Zudem schließen sie die Möglichkeit organisationaler Macht- und Kontrollausübung eher aus; Macht wird tendenziell der institutionellen Umwelt zugeschrieben und nicht der Organisation (DiMaggio/Powells 1991). Die RDT betont hingegen die für Organisationen bestehende Notwendigkeit, sich an die Unsicherheiten der Umwelt anzupassen, Ressourcenflüsse aktiv zu verwalten und bestenfalls zu kontrollieren, um mit problematischen Interdependenzen umgehen zu können (Pfeffer/Salancik 1978). Innerhalb der RDT wird somit davon ausgegangen, dass Organisationen auf die Ressourcenumwelt und die Austauschpartner Kontrolle und Einfluss ausüben können.
} 
Umweltanforderungen bezieht. Trotz umfasst die öffentliche Abwehr und Verurteilung institutioneller Konformitätszwänge und die Manipulation beinhaltet die Einbindung, Beeinflussung und Kontrolle von Akteuren aus der Umwelt auch hinsichtlich der Formung von normativen Maßstäben (Tab. 4.4).

Die im Kontext von negativen Zuschreibungen und Diskursen über migrantische Vereinigungen möglichen diametralen Reaktionen - Rückzug bzw. Resignation einerseits und offensive Vorstöße andererseits - könnten den Strategien der Vermeidung oder des Trotzes im Sinne eines Protestes zugeordnet werden.

Tab. 4.5 Strategische Reaktionen auf institutionelle Umwelteinflüsse

\begin{tabular}{|c|c|c|c|}
\hline Strategien & Taktiken & Beispiele für Taktiken & $\begin{array}{l}\text { Einordnung nach } \\
\text { den drei empi- } \\
\text { risch validierten } \\
\text { Mechanismen von } \\
\text { Rosenow-Williams } \\
\text { (2012: } 457 \mathrm{ff} \text {.) }\end{array}$ \\
\hline 1. Duldung & $\begin{array}{l}\text { a. Gewöhnen } \\
\text { b. Imitieren } \\
\text { c. Befolgen }\end{array}$ & $\begin{array}{l}\text { - Als selbstverständlich geltende } \\
\text { Normen befolgen } \\
\text { - Institutionalisierte Modelle nach- } \\
\text { ahmen } \\
\text { - Regeln befolgen und Normen } \\
\text { akzeptieren }\end{array}$ & Isomorphie \\
\hline 2. Kompromiss & $\begin{array}{l}\text { a. Ausgleichen } \\
\text { b. Befrieden } \\
\text { c. Verhandeln }\end{array}$ & $\begin{array}{l}\text { - Erwartungen verschiedener } \\
\text { Akteure ausgleichen } \\
\text { - Besänftigen, institutionalisierte } \\
\text { Elemente anpassen } \\
\text { - Mit den „stakeholdern“ in } \\
\text { Verhandlung treten }\end{array}$ & \multirow{2}{*}{ Entkopplung } \\
\hline 3. Vermeidung & $\begin{array}{l}\text { a. Verbergen } \\
\text { b. Puffern } \\
\text { c. Fliehen }\end{array}$ & $\begin{array}{l}\text { - Nichtkonformitität verstecken } \\
\text { - Anknüpfung zur institutionellen } \\
\text { Umwelt lockern } \\
\text { - Ziele, Aktivitäten oder Wirkungs- } \\
\text { bereiche verändern }\end{array}$ & \\
\hline 4. Trotz & $\begin{array}{l}\text { a. Zurückweisen } \\
\text { b. Herausfordern } \\
\text { c. Angreifen }\end{array}$ & $\begin{array}{l}\text { - Explizite Normen und Werte } \\
\text { ignorieren } \\
\text { - Regeln und Anforderungen } \\
\text { anfechten } \\
\text { - Quellen institutioneller } \\
\text { Zwänge angreifen }\end{array}$ & \multirow{2}{*}{ Protest } \\
\hline 5. Manipulation & $\begin{array}{l}\text { a. Kooptieren } \\
\text { b. Beeinflussen } \\
\text { c. Steuern }\end{array}$ & $\begin{array}{l}\text { - Einflussreiche Akteure einbinden } \\
\text { - Werte und Kriterien entwickeln } \\
\text { und formen } \\
\text { - Institutionelle Prozesse und } \\
\text { Akteure beherrschen }\end{array}$ & \\
\hline
\end{tabular}

Quelle: Walgenbach/Meyer 2008: 124; Oliver 1991: 152; Rosenow-Williams 2012: 68 ff., 457 ff. 
Im Zusammenhang mit ihrer Typologie stellt Christine Oliver (1991: 147) sechs Annahmen auf:

1) Organisationale Wahl- und Entscheidungsmöglichkeiten werden durch multiple externe Zwänge eingeschränkt.

2) Die Umwelt einer Organisation besteht aus miteinander verflochtenen Kollektiven.

3) Das Überleben einer Organisation hängt von der Reaktion auf externe Anforderungen und Erwartungen ab.

4) Organisationen streben nach Stabilität und Vorhersehbarkeit sowie

5) nach Legitimität.

6) Organisationen agieren interessengesteuert.

Auf Basis dieser Hypothesen justiert Oliver die Eintrittswahrscheinlichkeiten der verschiedenen organisationalen Reaktionen. Herauszustellen ist, dass die jeweiligen Realisierungswahrscheinlichkeiten spezifischer Antworten auf von außen einwirkende Erwartungshaltungen und die (Nicht-)Konformität mit letzteren auch von der Einschätzung der externen Verhältnisse durch die Organisationen abhängen. So können Kooperationsbereitschaften z. B. beeinträchtigt werden, wenn eine Organisation die Verweigerung, Nicht-Verfügbarkeit oder ein zu geringes Ausmaß der erwünschten, von außen zu erlangenden Ressourcen von Legitimität, Ansehen oder finanzieller Unterstützung antizipiert oder ein zu großer Vielfältigkeitsgrad von Anforderungen besteht. Auch wenn interne Interessen und Ziele in einem starken Widerspruch zu den wahrgenommenen externen Forderungen stehen oder der Umfang der (nach Organisationsermessen) bestehenden Beschränkungen zu groß ausfällt, wird dies den Kooperationswillen unterminieren (Oliver 1991: 160 ff.; Rosenow-Williams 2012: 455). Oliver betont zudem, dass die verschiedenen Arten von Reaktionen der Organisationen auch von deren Fähigkeit und Motivation abhängen, Macht und externen Druck auszuüben (Oliver 1991: 150), was insbesondere auch im Rahmen ihrer Ausführungen zur Manipulierungsstrategie ersichtlich wird (ebd.: 157 f., 162).

Die Anschlussfähigkeit der (weiter-)entwickelten theoretischen Annahmen des NI an die Empirie insbesondere in Bezug auf umweltbedingte organisationale Reaktionsstrategien hat Kerstin Rosenow-Williams (2012; Rosenow 2010) nachgewiesen, indem sie die drei islamischen Dachverbände DİTİB, Millî Görüş und den Zentralrat der Muslime in Deutschland (ZMD) aus neoinstitutionalistischer Perspektive analysiert hat. Auf Basis umfangreicher Dokumentenanalysen und Experteninterviews mit Verbandsvertretern geht sie der Forschungsfrage nach, wie und warum islamische Dachverbände mit ihrem organisationalen Feld 
interagieren. Sie zeigt auf, dass die Handlungsmuster der Dachverbände von den wahrgenommenen internen und externen Erwartungen gesteuert werden. Rosenow-Williams identifiziert drei Handlungsstrategien: Anpassung (Isomorphie), Entkopplung und Protest. Die Proteststrategie stellt die stärkste Form des Widerstandes gegenüber institutionellen Erwartungen dar und findet sich nicht in den neoinstitutionalistischen Konzeptualisierungen wieder. Rosenow-Williams zufolge stellt sie eine Kombination aus der Trotz- und Manipulationsstrategie von Oliver dar (Tab. 4.4), die notwendig wird und den Organisationen als legitim erscheint, wenn Mitgliederinteressen gegen mit diesen höchst inkompatiblen Erwartungen von außen verteidigt werden müssen (Rosenow-Williams 2012: 75) und externe Anreize für Konformität nur sehr begrenzt ausfallen (ebd.: 465).

Rosenow-Williams resümiert, dass ihre Untersuchung des Auftretens der großen islamischen Verbände innerhalb des organisationalen Feldes eine über die Annahmen des NI hinausgehende organisationale Flexibilität und längst nicht nur Prozesse der gegenseitigen Anpassung zwischen den Hauptakteuren zeigt (ebd.: 456). Stattdessen sei neben einer reinen Erscheinungsform der idealtypischen Reaktionsstrategien von Anpassung, Entkopplung und Protest bei den untersuchten Verbänden auch eine Kombination der Mechanismen zu beobachten. Erkennbar sei einerseits, dass das vorhandene Interesse an einer weiteren offiziellen Anerkennung seitens der Verbände deren Anpassung an politische Erwartungen durchaus forciere; andererseits würde eine (gesellschafts-)politische Verweigerung oder Nicht-Verfügbarkeit von Legitimität, finanzieller Unterstützung und Status die Kooperationsbereitschaft der Verbände erheblich beeinträchtigen (ebd.: 460 ff.). Darüber hinaus würde deren Kooperationswillen unterminiert, wenn interne Interessen in einem starken Widerspruch zu den wahrgenommenen externen Forderungen stünden (ebd.: 455).

Vor dem Hintergrund der in diesem Kapitel dargelegten theoretischen Ansätze und empirischen Befunde werden abschließend insgesamt vier auf das Kooperationsverhalten der befragten MSO und deren Streben nach Legitimität, Status und Ressourcenzufluss bezogene Hypothesen formuliert.

Im Kontext der erläuterten Annahmen ist argumentiert worden, dass die Wahrnehmung von pejorativen Zuschreibungen und öffentlichen Negativ-Diskursen einflussreiche Faktoren sind, die sich auch auf die Kooperationsbereitschaft der MSO auswirken werden. Einige werden die Zusammenarbeit mit anderen Organisationen suchen, während sich andere mit großer Wahrscheinlichkeit zurückziehen, sich gegenüber der Umwelt abgrenzen und Kooperationen meiden bzw. keine kooperativen Strategien verfolgen. Daran anknüpfend wird folgende Doppelhypothese formuliert: 
H51: Die Wahrnehmung von Fremdenfeindlichkeit und Diskriminierung führt mit großer Wahrscheinlichkeit dazu, dass sich MSO zurückziehen und keine Kooperationen eingehen.

$\mathbf{H 5}_{2}$ : Die Wahrnehmung von negativen öffentlichen Zuschreibungen und Diskursen bewirkt mit großer Wahrscheinlichkeit, dass MSO verstärkt Kooperationen eingehen.

In Hinblick auf die im Rahmen von Kooperationen mit anderen Organisationen konkret verfolgten Ziele wird entsprechend des NI angenommen, dass es ein zentrales Anliegen der migrantischen Vereinigungen ist, einen Legitimitätsstatus - im Sinne von Integrität, Vertrauenswürdigkeit und Anerkennung - zu erlangen. Sie operieren und präsentieren sich daher entsprechend, um seitens für sie zentraler Akteure und Anspruchsgruppen Wertschätzung, Zustimmung und Unterstützung zu erhalten. An dieser theoretischen Prämisse aufsetzend wird folgende Hypothese formuliert:

H6: Verglichen mit anderen Anliegen stellen v. a. die Hervorhebung des Umfangs und der Nützlichkeit der Aktivitäten, die Steigerung der Bekanntheit und Vernetzung der MSO, der Abbau von wahrgenommenen Vorbehalten sowie die Verbesserung der Kontakte zu Politikern zentrale Kooperationsziele dar.

Vor dem Hintergrund der Annahme vom im organisationalen Feld zwischen den MSO bestehenden diversen Konkurrenzverhältnissen um finanzielle Ressourcen, Mitglieder, Zielgruppen, politische Legitimität und öffentliche Anerkennung wird eine weitere, letzte Hypothese aufgestellt:

H7: Wettbewerb belebt das Kooperationsgeschehen: MSO, die andere MSO als Konkurrenten betrachten, arbeiten nicht mit anderen MSO, sondern v. a. mit städtischen und staatlichen Behörden zusammen.

Mit dem Fragebogen als Erhebungsinstrument kann jedoch nicht herausgefunden werden, inwieweit (nicht-)vorhandenes Kooperationsverhalten auf spezifische Strategien und mit solchen einhergehende Taktiken der MSO zurückzuführen sind. ${ }^{86}$ Die Zuordnung zu bestimmten Strategien oder Taktiken ist aber

\footnotetext{
${ }^{86}$ So kann nicht ermittelt werden, ob und inwieweit möglicherweise festzustellende Zusammenhänge zwischen wahrgenommener Fremdenfeindlichkeit bzw. Diskriminierung oder Negativ-Diskursen und dem Nicht-Kooperieren von MSO deren Protest impliziert, indem
} 
auch nicht relevant. Bedeutsam ist vielmehr, ob Zusammenhänge zwischen den Merkmalsausprägungen festzustellen sind und wie sie sich deuten lassen.

\subsection{Die theoretischen Zugänge: Eine Bilanz}

Eine Organisation stellt ein eigenständiges soziales System dar, das von Menschen konstituiert wird. In Organisationen wird kommuniziert und gemeinsam gehandelt, sie besitzen spezifische Funktionen und Zwecke. Die in dieser Arbeit verfolgten theoretischen Ansätze haben verdeutlicht, dass man bei der Beschäftigung mit organisationalen Performanzen nicht von der Individualebene abstrahieren kann. Es sind die Individuen, die sich mit je eigenen Bedürfnissen, Interessen, Merkmalen und Qualifikationen in das Sozialgebilde Organisation einbringen und die als „Kollektiv“ gemeinsam tätig sind. Dieses Sozialgebilde ist in seiner Existenz von der Bereitschaft und Motivation der Menschen abhängig, sich einbinden zu lassen und damit eine Rolle zu übernehmen, die mit ihrer Eigenschaft als Mitglied verbunden ist. Dies bedeutet gerade nicht, dass das kollektive Handeln von einer abstrakten metaphysischen Kraft quasi automatisch fremdgesteuert wird. Vielmehr wurde dargelegt, dass das Miteinander-Auskommen, innerorganisationale Ordnung, Gruppenbindung und Anpassungen anhand von Regeln und im Rahmen gruppensoziologischer Prozesse reguliert wird; regelabweichendes Verhalten kann innerhalb der Gruppe auf unterschiedliche Art sanktioniert werden.

Aufgrund der bewussten freiwilligen Entscheidung für Mitgliedschaft und Engagement ist davon auszugehen, dass dem Individuum die Zugehörigkeit zu einer bestimmten Vereinigung wichtig ist und dass es sich mit ihren Zielen weitgehend identifizieren kann. Insbesondere in gesellschaftlich gegebener Minderheitensituation kann das Eingebundensein in eine Gemeinschaft - via kollektiver Identität und „Wirgefühl“ - die subjektive soziale Identität, das Selbstbewusstsein, schließlich auch das Sicherheitsgefühl stärken. Die Erfahrung von Zusammengehörigkeit und Solidarität kann mit (internalisiertem) sittlich-moralischem Verantwortungsgefühl einhergehen, das für Mitgliedschaft und Engagement einen starken Motivator darstellt. Dabei ist davon auszugehen, dass Organisationen multiple, zugleich selektive Anreize offerieren und dass (potenzielle) Mitglieder und Engagierte ihre Entscheidungen nicht ausschließlich entlang klar definierbarer

\footnotetext{
z. B. diese Fremdzuschreibungen öffentlich zurückgewiesen oder attackiert werden, oder ob und auf welche Art ggf. gegen sie angekämpft wird. Ebenso kann nicht ausfindig gemacht werden, inwieweit z. B. das mit einer Kooperation einhergehende Ziel der Verbesserung der Kontakte zu Politkern der von Oliver (1991) identifizierten Strategie des Kompromisses oder eher der Strategie der Manipulation entspricht (Tab. 4.5).
} 
Kosten-Nutzen-Erwägungen treffen. Denn Motivkräfte sind auch intrinsischer, sozialer und wertorientierter Natur, die auf breiter Basis anzusiedeln sind: Sie umfassen beispielsweise emotional-gesellige Aspekte, Gerechtigkeitsdenken, den Wunsch nach sozialer Anerkennung und gemeinsamen Wertschätzungen und führen in bestimmten Situationen, in Anbetracht verfügbarer Mittel, zu Entscheidungen, die die Erreichung eines erwünschten Zielzustands hinreichend in Aussicht stellen.

Insbesondere religiöse Gemeinschaften bzw. Vereinigungen bieten Angebote für Gemeinsamkeit und kollektive Identität; mehr noch sind sie für das gläubige Individuum und seine religiös orientierte Lebensführung zentral, da Glaube und religiöse Identität in diese eingebettet sind, durch die Zusammenkunft mit anderen religiös geprägten Menschen erst plausibilisiert, kommunikabel und erfahrbar werden. Insofern benötigt religiöse Identität im Grunde den Austausch mit anderen religiösen Menschen.

Religiöse Orientierungen und sittlich-religiöse Weisungen („Liebe Deinen Nächsten wie Dich selbst") können in besonderem Maß Engagement stimulieren, das einerseits die Gruppe nach innen stärkt, das andererseits aber auch Brücken zu Andersgläubigen oder Nicht-Religiösen bauen kann, z. B. im Rahmen interreligiöser oder sozialarbeiterischer Projekte. Jedoch setzt dies eine gewisse Offenheit voraus. Denn wenn religiöse Glaubenshaltungen und Wertvorstellungen mit einem exklusivistischen Wahrheitsanspruch, dogmatisch-apodiktischen Auslegungen heiliger Schriften, mit Tendenzen zur Abwertung oder Verdammung von Anders- oder Nicht-Gläubigen verknüpft werden, ist die Gefahr von Abschottung unmittelbar gegeben. Auch die Unterordnung subjektiver unter kollektive Identität wie auch der soziale Druck, gruppenkonform zu handeln, sind Abgrenzung und Isolierung zweifellos zuträglich.

Religionen bergen insofern immer ambivalente Ausrichtungen, Spannungsverhältnisse zwischen Innen- und Außenwelt. Spezifische Eigenschaften, die religiöse Vereinigungen und ihre Mitglieder nach innen stark machen, können sozial erwünschte Wirkungen entfalten, Integration und gesellschaftliche Beteiligung aber auch behindern. Religiöse Bindungen können die (Zivil-)Gesellschaft und Demokratie stärkendes Sozialkapital produzieren, gesellschaftspolitisch aber auch irrelevant oder sogar schädlich sein, wenn sie sich intolerant oder verfassungsfeindlich gerieren. Konfessionen können sich somit von zwei völlig gegensätzlichen Seiten zeigen, wodurch sie potenziell, doppelgesichtig 'sind. Die Thematisierung dieser inhärenten Gefahr zieht sich wie ein roter Faden durch die die MSO betreffenden Debatten, denn die gesellschaftspolitische Forderung lautet, integrative Leistungen $\mathrm{zu}$ erbringen. Gleichwohl muss bedacht werden, 
dass zum Zweck von Kultur- oder Religionspflege gegründete Vereine - migrantischer wie nicht-migrantischer Provenienz - auch um ihrer selbst willen unter sich bleiben wollen, was keineswegs automatisch mit Abschottung und Integrationsverweigerung zu tun haben muss. Zugleich können Diskriminierungserfahrungen Rückzüge provozieren, wenn die Gemeinschaft Schutz und emotional-psychische Stabilisierung bei als bedrohlich eingestuften Umweltbedingungen verspricht.

Ressourcen - personelle wie materielle -, die erfolgreich mobilisiert werden können bzw. müssen, bestimmen die Handlungsfähigkeit der Organisationen in ermöglichender oder beschränkender Weise. Das Gleiche gilt für (politische) Kontextbedingungen, die je nach gegebenen Konstellationen als eher vorteilhaft oder unvorteilhaft einzustufen sind. Austausch mit der Umwelt findet entsprechend der Mitgliederinteressen sowie - wahrgenommener oder faktisch bestehender - externer Erwartungshaltungen und Abhängigkeiten statt. Diese müssen berücksichtigt und ausbalanciert werden, will eine Organisation Legitimität, Anerkennung und öffentliche Förderung erhalten. Damit besteht ein Spannungsverhältnis zwischen dem Streben einer Organisation, aufgrund eines als notwendig erachteten Bedarfs an Ressourcenzuflüssen auf ihre Umwelt einzuwirken einerseits (,Einflusslo-

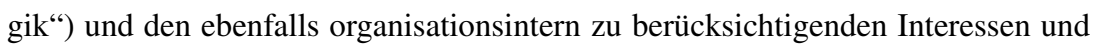
Forderungen der Mitglieder andererseits (,Mitgliedschaftslogik“).

Für das Management von Interdependenzen können Organisationen auf unterschiedliche Strategien zurückgreifen: eher passiv auf das Handeln anderer Organisationen reagieren, sich anpassen, Kompromisse eingehen (,entkoppeln“), protestieren und offensive Maßnahmen ergreifen, um auf ihre Umwelt Einfluss zu nehmen und Abhängigkeitsbeziehungen so zu gestalten, dass sie ihre Autonomie erhöhen und damit ihre Interessen durchsetzen können. Der zentralen Annahme des Neoinstitutionalismus zufolge stellt das Streben nach sozialer Anerkennung das eigentliche Leitprinzip dar, über das sich die Performanz von Organisationen definiert. Wie verdeutlicht wurde, ist allerdings von sehr unterschiedlichen organisationalen Reaktionsmöglichkeiten auszugehen.

Insbesondere bei wahrgenommener Differenz zwischen Erwartungen und Realität, Diskrepanz zwischen Ist- und erwünschtem Soll-Zustand, können große Organisationen, wie islamische Interessenverbände - je nach Einflussmöglichkeiten - neue Partizipationsmöglichkeiten einfordern, um bestimmte Themen und als misslich wahrgenommene Zustände auf die politische Agenda zu setzen. Die Registrierung von erforderlichen Verbesserungen basiert auf kollektiver Deutung (,framing“), die (strategisches) Engagement stimulieren und legitimieren kann. 
Formale Zusammenschlüsse bestimmter sozialer Gruppen mit je spezifischen Zielen, Strukturen und Aktivitätsformen ermöglichen den involvierten Personen zu eigener Bedürfnisbefriedigung zusammenzukommen und gemeinsam effektiv(er) handeln zu können; zugleich sind die Mitglieder eine elementare, da konstitutive Ressource für die Organisation als Kollektivakteur, die ohne Mitglieder schlichtweg nicht existieren und handlungsfähig werden kann.

Open Access Dieses Kapitel wird unter der Creative Commons Namensnennung 4.0 International Lizenz (http://creativecommons.org/licenses/by/4.0/deed.de) veröffentlicht, welche die Nutzung, Vervielfältigung, Bearbeitung, Verbreitung und Wiedergabe in jeglichem Medium und Format erlaubt, sofern Sie den/die ursprünglichen Autor(en) und die Quelle ordnungsgemäß nennen, einen Link zur Creative Commons Lizenz beifügen und angeben, ob Änderungen vorgenommen wurden.

Die in diesem Kapitel enthaltenen Bilder und sonstiges Drittmaterial unterliegen ebenfalls der genannten Creative Commons Lizenz, sofern sich aus der Abbildungslegende nichts anderes ergibt. Sofern das betreffende Material nicht unter der genannten Creative Commons Lizenz steht und die betreffende Handlung nicht nach gesetzlichen Vorschriften erlaubt ist, ist für die oben aufgeführten Weiterverwendungen des Materials die Einwilligung des jeweiligen Rechteinhabers einzuholen. 